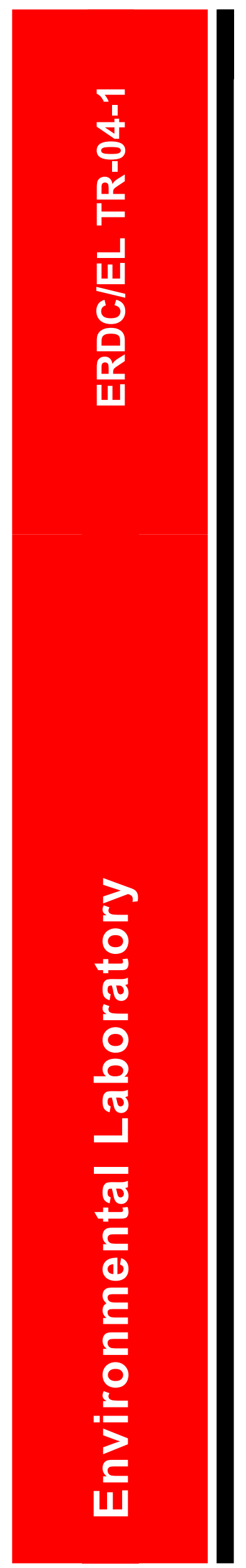

Environmental Security Technology Certification Program

\title{
Advanced UXO Detection/Discrimination Technology Demonstration - Kaho'olawe, Hawaii
}

Diane M. Cargile, Hollis H. Bennett, Ricky A. Goodson, March 2004

\author{
Tere' A. DeMoss, and Ernesto R. Cespedes
}

US Army Corps of Engineers ${ }_{\circledast}$

Engineer Research and Development Center 


\section{Advanced UXO Detection/Discrimination Technology Demonstration - Kaho'olawe, Hawaii}

Diane M. Cargile, Hollis H. Bennett, Ricky A. Goodson, Tere' A. DeMoss, Ernesto R. Cespedes

Environmental Laboratory

U.S. Army Engineer Research and Development Center

3909 Halls Ferry Road

Vicksburg, MS 39180-6199

Final report

Approved for public release; distribution is unlimited

Prepared for Environmental Security Technology Certification Program Office Arlington, VA 22203

and Headquarters, U.S. Army Corps of Engineers

Washington, DC 20314-1000

Under Project 200034 and Project AF-25 


\begin{abstract}
The primary technical objective of this demonstration project was to evaluate the detection and discrimination capabilities (including production rates and costs) of advanced UXO systems in difficult magnetic clutter environments such as those encountered at Kaho'olawe, Hawaii. One 90-m by $111.1-\mathrm{m}$ (1-hectare) area and 10 (not necessarily contiguous) $30-\mathrm{m}$ by $30-\mathrm{m}$ test grids within the Kaho'olawe Quality Assurance (QA) Range were prepared to present a limited range of target/clutter/ topography/vegetation/magnetic background conditions to the various demonstrators' systems: Geonics EM-63, GTL TM-5 EMU, Geophex GEM-3, NRL EMMS, and Geonics EM-61. Anomaly maps, survey maps, and demonstrators target discrimination charts are compared to actual groundtruth to determine performance assessment of detection, discrimination, and false alarm rate. At Kaho'olawe, the advanced EMI systems did not demonstrate significant performance and/or cost improvements over the baseline technology consisting of a standard EM-61 system operated in an "EM and Flag" mode. This was not true at Jefferson Proving Ground, Indiana (July 2000). Finally, the safety and logistics problems associated with conducting technology demonstrations concurrent with actual UXO cleanup operations proved to be a very inefficient, costly, and time-consuming process.
\end{abstract}

DISCLAIMER: The contents of this report are not to be used for advertising, publication, or promotional purposes. Citation of trade names does not constitute an official endorsement or approval of the use of such commercial products. All product names and trademarks cited are the property of their respective owners. The findings of this report are not to be construed as an official Department of the Army position unless so designated by other authorized documents. 


\section{Contents}

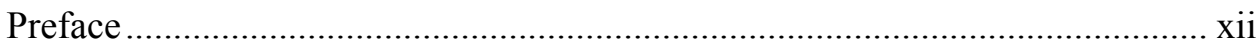

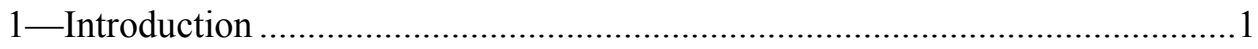

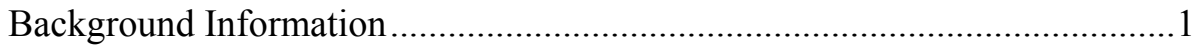

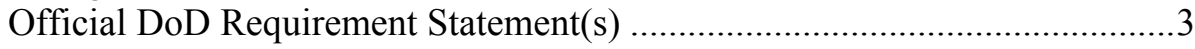

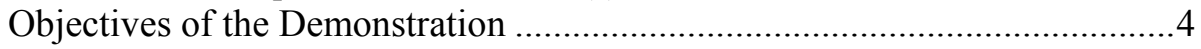

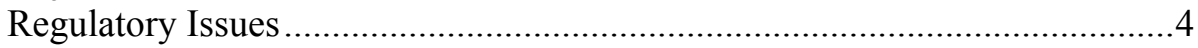

Previous Testing of the Technology ……….................................................

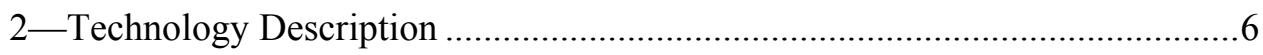

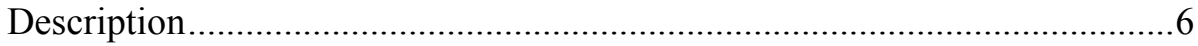

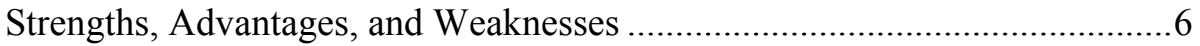

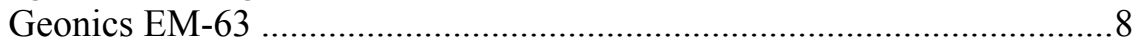

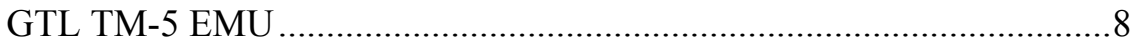

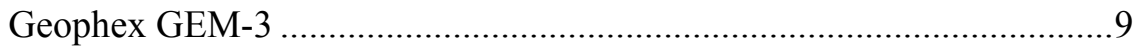

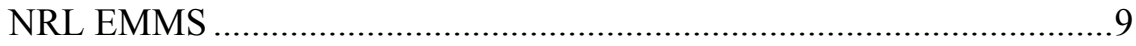

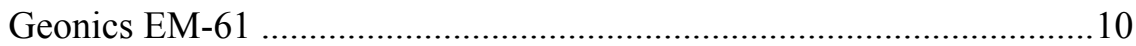

Factors Influencing Cost and Performance ..............................................10

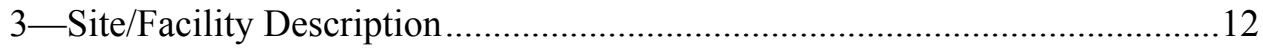

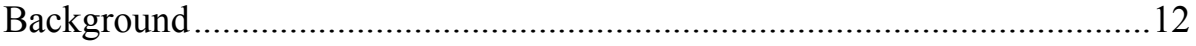

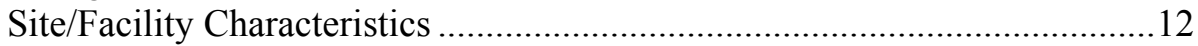

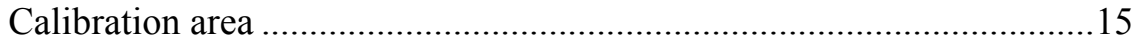

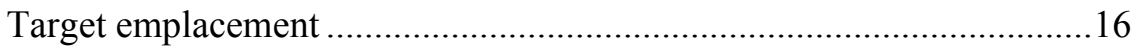

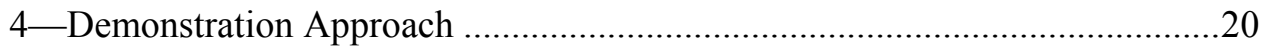

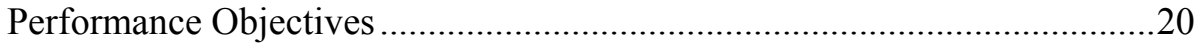

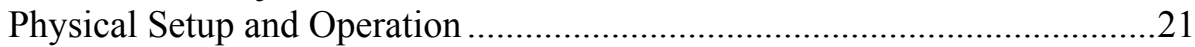

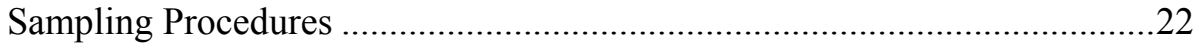

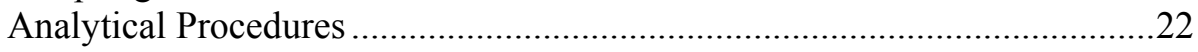

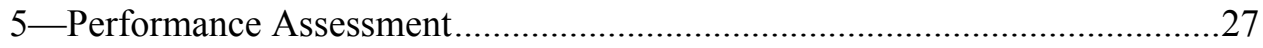

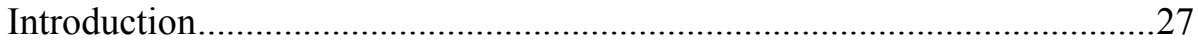

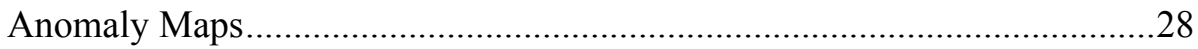

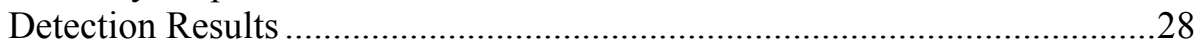

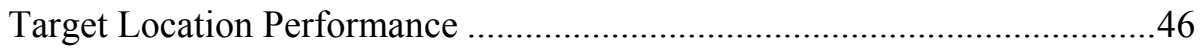

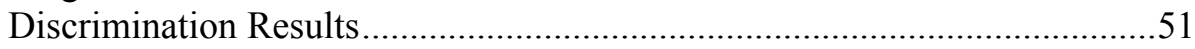

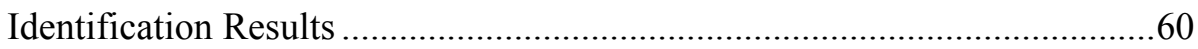

ROC-Based Performance Assessment .........................................................62 
Assessment of detection, discrimination, and false alarm rate performance

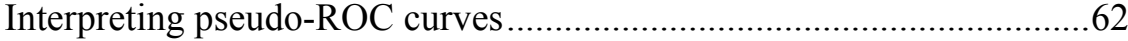

Technology Comparison ......................................................................... 72

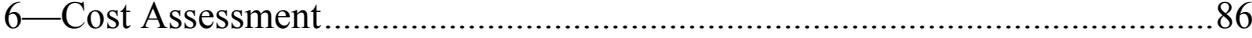

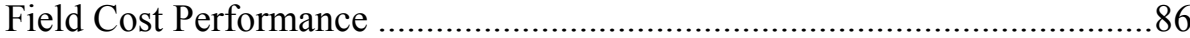

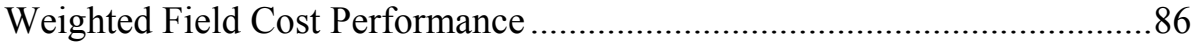

7-Regulatory Issues -Compliance and Acceptance ..........................................89

8-Technology Transition/ Implementation ......................................................90

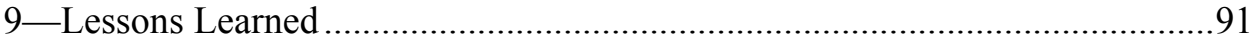

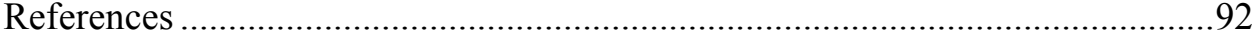

Appendix A: Points of Contact …..............................................................

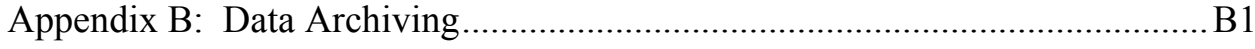

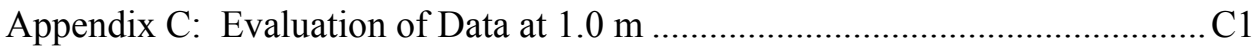

Appendix D: Mean Location Error Analysis.................................................... 1

Appendix E: Demonstrators' Data Collection and Analysis Plans .................... E1

SF 298

\section{List of Figures}

Figure 1. Overview of Kaho'olawe Island..................................................

Figure 2. EM-63 operated by NAEVA _................................................

Figure 3. TM-5 EMU operated by GTL …........................................... 7

Figure 4. GEM-3 operated by Geophex, Ltd.........................................

Figure 5. $\quad$ EMMS operated by NRL …...............................................

Figure 6. $\quad$ EM-61 operated by Parsons ....................................................

Figure 7. TM-5 EMU operated by Parsons..................................................

Figure 8. Overview of Kaho'olawe showing erosion of terrain ...............13

Figure 9. Overview of Kaho'olawe vegetation and hardpan terrain..........13

Figure 10. Topography map with outline of Base Camp, calibration, and demonstration ranges ....................................................... 14

Figure 11. Calibration area within the QC range......................................14

Figure 12. Layout of demonstration areas ............................................... 15

Figure 13. Photo of Demonstration area within the QA range ....................16 
Figure 14. Kaho'olawe calibration area target emplacement plan

Figure 15. Kaho'olawe site map showing emplaced items in Areas $\mathrm{A}, \mathrm{B}$, and $\mathrm{C}$ of the demonstration site .19

Figure 16. NAEVA EM-63 survey of Area A ...........................................29

Figure 17. NAEVA EM-63 survey of Area B ............................................30

Figure 18. NAEVA EM-63 survey of Area C ........................................... 31

Figure 19. GTL TM-5 EMU survey of Area A …......................................32

Figure 20. GTL TM-5 EMU survey of Area B.......................................... 32

Figure 21. GTL TM-5 EMU survey of Area C..........................................33

Figure 22. Geophex, Ltd. GEM-3 survey of Areas A, B, and C...................35

Figure 23. NRL EMMS survey of Areas A, B, and C ...............................36

Figure 24. Parsons EM and Flag survey of Area A ….................................37

Figure 25. Parsons EM and Flag survey of Area B ....................................38

Figure 26. Parsons EM and Flag survey of Area C .....................................39

Figure 27. Parsons EM-61 Digital survey of Area B.................................40

Figure 28. Parsons EM-61 Digital survey of Area C..................................41

Figure 29. Parsons TM-5 EMU survey of Area A......................................42

Figure 30. Parsons TM-5 EMU survey of Area B .....................................43

Figure 31. Parsons TM-5 EMU survey of Area C ......................................4

Figure 32. NAEVA EM-63 target identification matrix for Area A within $0.5 \mathrm{~m}$..

Figure 33. NAEVA EM-63 target identification matrix for Area B within $0.5 \mathrm{~m}$

Figure 34. NAEVA EM-63 target identification matrix for Area C

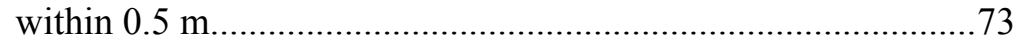

Figure 35. NAEVA EM-63 size classification matrix within 0.5 m...........66

Figure 36. Geophex, Ltd. GEM-3 size classification matrix within $0.5 \mathrm{~m}$.

Figure 37. Area $\mathrm{A}-\mathrm{P}(\mathrm{det}) \mathrm{vs}$. total FAR within $0.5 \mathrm{~m}$ for all demonstrators .....

Figure 38. Area B $-\mathrm{P}(\mathrm{det}) \mathrm{vs}$. total FAR within $0.5 \mathrm{~m}$ for all demonstrators .70 
Figure 39. Area $\mathrm{C}-\mathrm{P}(\mathrm{det}) \mathrm{vs}$. total FAR within $0.5 \mathrm{~m}$ for all demonstrators

Figure 40. Area $\mathrm{A}-\mathrm{P}(\mathrm{det}) \mathrm{vs}$. total FAR within $0.5 \mathrm{~m}$ for demonstrators without (wo) $20 / 40 \mathrm{~mm}$

Figure 41. Area B $-\mathrm{P}(\mathrm{det}) \mathrm{vs}$. total FAR within $0.5 \mathrm{~m}$ for demonstrators wo $20 / 40 \mathrm{~mm}$

Figure 42. Area $\mathrm{C}-\mathrm{P}(\mathrm{det}) \mathrm{vs}$. total FAR within $0.5 \mathrm{~m}$ for demonstrators wo $20 / 40 \mathrm{~mm}$ .75

Figure 43. Area A wo 20/40 mm - P(det) vs. total FAR within $0.5 \mathrm{~m}$ for all demonstrators

Figure 44. Area B wo 20/40 mm - P(det) vs. total FAR within $0.5 \mathrm{~m}$ for all demonstrators.

Figure 45. Area C wo 20/40 mm - P(det) vs. total FAR within $0.5 \mathrm{~m}$ for all demonstrators

Figure 46. Area A - P(det) vs. Pfp within $0.5 \mathrm{~m}$ for all demonstrators

Figure 47. Area B - P(det) vs. Pfp within $0.5 \mathrm{~m}$ for all demonstrators

Figure 48. Area $\mathrm{C}-\mathrm{P}(\mathrm{det}) \mathrm{vs}$. Pfp within $0.5 \mathrm{~m}$ for all demonstrators

Figure 49. Area A - Pdisc (prioritized by signal strength) vs. total FAR within $0.5 \mathrm{~m}$ for all demonstrators.

Figure 50. Area B - Pdisc (prioritized by signal strength) vs. total FAR within $0.5 \mathrm{~m}$ for all demonstrators.

Figure 51. Area $\mathrm{C}-$ Pdisc (prioritized by signal strength) vs. total FAR within $0.5 \mathrm{~m}$ for all demonstrators

Figure C1. NAEVA EM-63 target identification matrix for Area A within $1.0 \mathrm{~m}$.

Figure C2. NAEVA EM-63 target identification matrix for Area B within $1.0 \mathrm{~m}$.

Figure C3. NAEVA EM-63 target identification matrix for Area C within $1.0 \mathrm{~m}$.

Figure C4. NAEVA EM-63 size classification matrix within 1.0 m.........C22

Figure C5. Geophex, Ltd. GEM-3 size classification matrix within $1.0 \mathrm{~m}$.

Figure C6. Area A-P(det) vs. total FAR within $1.0 \mathrm{~m}$ for all demonstrators $\mathrm{C} 24$ 
Figure C7. Area B - P(det) vs. total FAR within $1.0 \mathrm{~m}$ for all demonstrators

Figure C8. Area $\mathrm{C}-\mathrm{P}(\mathrm{det})$ vs. total FAR within $1.0 \mathrm{~m}$ for all demonstrators

Figure C9. Area $\mathrm{A}-\mathrm{P}(\mathrm{det})$ vs. total FAR within $1.0 \mathrm{~m}$ for demonstrators without (wo) $20 / 40 \mathrm{~mm}$

Figure $\mathrm{C} 10$. Area $\mathrm{B}-\mathrm{P}(\mathrm{det}) \mathrm{vs}$. total FAR within $1.0 \mathrm{~m}$ for demonstrators wo $20 / 40 \mathrm{~mm}$

Figure $\mathrm{C} 11$. Area $\mathrm{C}-\mathrm{P}(\mathrm{det}) \mathrm{vs}$. total FAR within $1.0 \mathrm{~m}$ for demonstrators wo 20/40 $\mathrm{mm}$

Figure C12. Area A wo 20/40 mm - P(det) vs. total FAR within $1.0 \mathrm{~m}$ for all demonstrators.

Figure C13. Area B wo 20/40 mm - P(det) vs. total FAR within $1.0 \mathrm{~m}$ for all demonstrators

Figure C14. Area $\mathrm{C}$ wo $20 / 40 \mathrm{~mm}-\mathrm{P}(\mathrm{det})$ vs. total FAR within $1.0 \mathrm{~m}$ for all demonstrators.

Figure $\mathrm{C} 15 . \quad$ Area $\mathrm{A}-\mathrm{P}(\mathrm{det}) \mathrm{vs}$. Pfp within $1.0 \mathrm{~m}$ for all demonstrators

Figure C16. Area B - P(det) vs. Pfp within $1.0 \mathrm{~m}$ for all demonstrators

Figure C17. Area C - P(det) vs. Pfp within $1.0 \mathrm{~m}$ for all demonstrators

Figure $\mathrm{C} 18 . \quad$ Area $\mathrm{A}-\mathrm{P}($ disc) (prioritized by signal strength) vs. total FAR within $1.0 \mathrm{~m}$ for all demonstrators.

Figure C19. Area B - P(disc) (prioritized by signal strength) vs. total FAR within $1.0 \mathrm{~m}$ for all demonstrators.

Figure $\mathrm{C} 20$. Area $\mathrm{C}-\mathrm{Pdisc}$ (prioritized by signal strength) vs. total FAR within $1.0 \mathrm{~m}$ for all demonstrators. C39

Figure D1. Area A - Mean location error by emplaced item ..................... D2

Figure D2. Area B - Mean location error by emplaced item ......................D3

Figure D3. Area C-Mean location error by emplaced item ......................D4

Figure D4. Area A - Mean location error by demonstrator......................... D5

Figure D5. Area B - Mean location error by demonstrator.........................D6

Figure D6. Area C - Mean location error by demonstrator.........................D7

Figure E1.1. Stacked profiles of test grid lane 0-gates 1:3:10 .....................E3

Figure E1.2. Basalt EM-63 Amplitude Response Decay $(\sim 1 / \mathrm{T}) \ldots \ldots \ldots \ldots \ldots \ldots . . . . . . . . .64$ 
Figure E1.3. Console comparison of drift .................................................. E5

Figure E1.4. Zeroing of the EM-63 before surveying ...................................E6

Figure E1.5. Traversing Kaho'olawe Demonstration Grid B with the EM-63 (Ropes at 2-m intervals) ......................................... 7

Figure E1.6. Site map of demonstration area .............................................. E7

Figure E1.7. Ordnance samples available on Kaho'olawe.............................E8

Figure E1.8. Check Ashtech GPS positions, Lane B18-28 .......................... E9

Figure E3.1. EM survey of the Kaho'olawe Calibration Grid ..................... E14

Figure E3.2. EM survey of the QA Site at Kaho'olawe..............................E15

Figure E3.3. Southwest Quadrant of the Calibration Site ............................E16

Figure E3.4. Calibration Site. Interpolated image from the magnetometry survey using a 15 -point demedian filter .......... E20

Figure E3.5. Pixel presentation of part of Grid 1E in the QA Site from the EM survey .................................................................... E21

Figure E3.6. QA Site, Grid 1C. Pixel presentation of the EM survey showing targets selected for analysis. E22

\section{List of Tables}

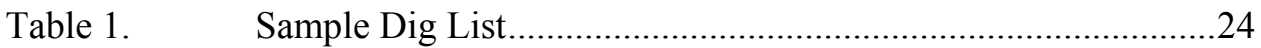

Table 2. $\quad \mathrm{P}(\mathrm{det})$ by Area within $0.5 \mathrm{~m}$..................................................28

Table 3. $\quad \mathrm{P}(\mathrm{det})$ by Area within $0.5 \mathrm{~m}$ and without $20 / 40 \mathrm{~mm}$.................45

Table 4. Grid-by-Grid Detections within $0.5 \mathrm{~m}$.....................................46

Table 5. UXO Found - NAEVA …..................................................... 47

Table 6. UXO Found - GTL .........................................................4

Table 7. UXO Found - Geophex \& Geophex without 20/40 mm............48

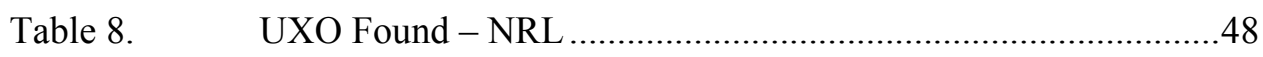

Table 9. UXO Found - NRL without 20/40 mm..................................49

Table 10. UXO Found - Parsons EM-61 EM \& Flag ...............................49

Table 11. UXO Found - Parsons EM-61 Digital .......................................50

Table 12. UXO Found - Parsons EM-61 TM-5 EMU …...........................50 
Table 13. UXO Target Location (x, y) Estimation Performance of the Demonstrators

Table 14. UXO Target Depth Estimation Performance of the

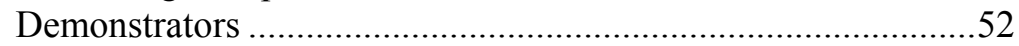

Table 15. Summary of Discrimination Performance...................................53

Table 16. Breakdown of Discrimination - QA Range and Area A -

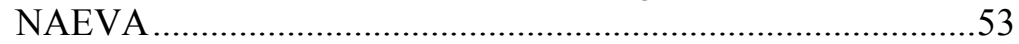

Table 17. Breakdown of Discrimination - Area B and Area C -

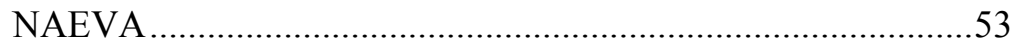

Table 18. Breakdown of Discrimination - QA Range - GTL....................54

Table 19. Breakdown of Discrimination - QA Range and Area A Geophex \& Geophex without 20/40 mm .................................54

Table 20. Breakdown of Discrimination - Area B and Area C Geophex \& Geophex without 20/40 mm ...................................55

Table 21. Breakdown of Discrimination - QA Range and Area A -

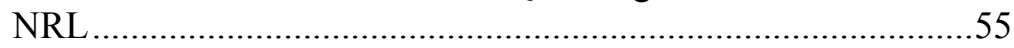

Table 22. Breakdown of Discrimination - Area B and Area C -

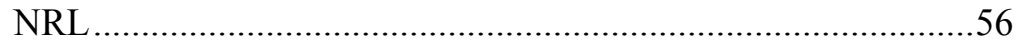

Table 23. Breakdown of Discrimination - QA Range and Area A NRL without $20 / 40 \mathrm{~mm}$......................................................56

Table 24. Breakdown of Discrimination - Area B and Area C NRL without 20/40 mm ...

Table 25. Breakdown of Discrimination - QA Range and Area A Parsons (EM61) EM and Flag ................................................57

Table 26. Breakdown of Discrimination - Area B and Area C Parsons (EM-61) EM and FLAG ...... .58

Table 27. Breakdown of Discrimination - QA Range and Area A Parsons EM-61 Digital ..............................................................58

Table 28. Breakdown of Discrimination - Area B and Area C Parsons EM-61 Digital.

Table 29. Breakdown of Discrimination - QA Range and Area A Parsons TM-5 EMU

Table 30. Breakdown of Discrimination - Area B and Area C Parsons TM-5 EMU .60

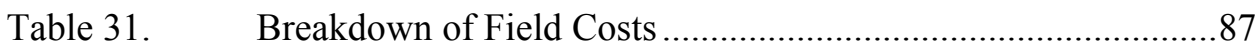

Table 32. Demonstrator Costs Including Penalties for False Alarms and for Leaving UXO Targets in Ground .88 


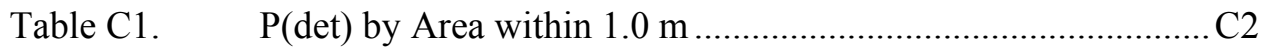

Table C2. $\quad \mathrm{P}(\mathrm{det})$ by Area within $1.0 \mathrm{~m}$ and without $20 / 40 \mathrm{~mm}$.................. 3

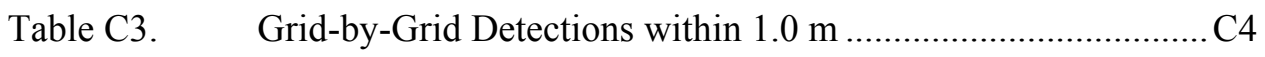

Table C4. UXO Found - NAEVA …............................................................

Table C5. UXO Found - GTL ............................................................. 5

Table C6. UXO Found - Geophex \& Geophex without 20/40 mm............ 5

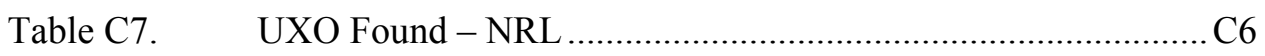

Table C8. UXO Found - NRL without 20/40 mm.................................... 6

Table C9. UXO Found - Parsons EM-61 EM \& Flag ............................... 7

Table C10. UXO Found - Parsons EM-61 Digital ...................................... C

Table C11. UXO Found - Parsons EM-61 TM-5 EMU …............................ 8

Table C12. Summary of Discrimination Performance................................. 9

Table C13. Breakdown of Discrimination - QA Range and Area A -

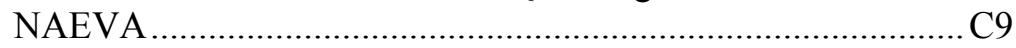

Table C14. Breakdown of Discrimination - Area B and Area C -

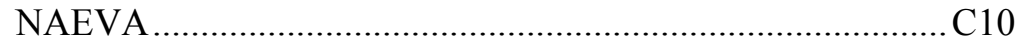

Table C15. Breakdown of Discrimination - QA Range - GTL................. 10

Table C16. Breakdown of Discrimination - QA Range and Area A -

Geophex \& Geophex without 20/40 mm ............................... 11

Table C17. Breakdown of Discrimination - Area B and Area C Geophex \& Geophex without 20/40 mm .

Table C18. Breakdown of Discrimination - QA Range and Area A -

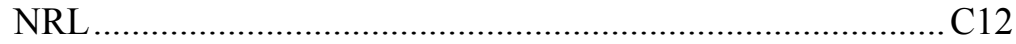

Table C19. Breakdown of Discrimination - Area B and Area C -

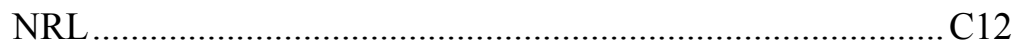

Table C20. Breakdown of Discrimination - QA Range and Area A NRL without 20/40 $\mathrm{mm}$

Table C21. Breakdown of Discrimination - Area B and Area C NRL without $20 / 40 \mathrm{~mm}$.

Table C22. Breakdown of Discrimination - QA Range and Area A Parsons (EM-61) EM and Flag. C14

Table C23. Breakdown of Discrimination - Area B - Parsons (EM-61) EM and Flag..... 
Table C24. Breakdown of Discrimination - QA Range and Area A Parsons EM-61 Digital ......................................................... 15

Table C25. Breakdown of Discrimination - Area B and Area C Parsons EM-61 Digital ............................................................ 15

Table C26. Breakdown of Discrimination - QA Range and Area A -

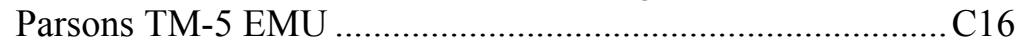

Table C27. Breakdown of Discrimination - Area B and Area C -

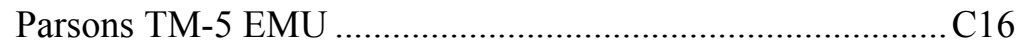

Table E3.1. MTADS TARGET REPORT (Edited), Kaho'olawe Calibration Site, 20-Point Smooth. E17 


\section{Preface}

The work documented in this report was performed during the period 3 September through 30 November 2001 as part of the Unexploded Ordnance (UXO) Technology Demonstration Program. This project was funded by the Department of Defense's Environmental Security Technology Certification Program (ESTCP) directed by Dr. Jeffrey Marqusee and managed by Dr. Anne Andrews, UXO Program Manager. Funding for the analysis documented in this report was provided by the Installation Restoration Research Program, Project AF-25, U.S. Army Corps of Engineers, directed by Dr. M. John Cullinane, Environmental Laboratory (EL), U.S. Army Engineer Research and Development Center (ERDC), Vicksburg, MS.

Mr. Hien Dinh, Naval Explosive Ordnance Disposal Technology Division, Indian Head, Maryland, had overall responsibility for the ESTCP project, was responsible for procuring and preparing the UXO test targets and emplaced clutter items used for this study, participated in the field demonstration activities, and was responsible for postdemonstration excavation and groundtruth verification activities. Dr. Ernesto R. Cespedes, EL, ERDC, was responsible for planning and coordinating the field demonstrations. He developed the metrics used to evaluate the performance of the demonstrators, participated in the site preparation and field demonstration activities, and directed the analysis of the results. Mr. George Robitaille, U.S. Army Environmental Center, was responsible for providing UXO targets from the UXO Standardized Test Site project inventory.

This project was performed under the general supervision of Dr. David Tazik, Chief, Ecosystems Evaluation and Engineering Division, and Dr. Elizabeth C. Fleming, Acting Director, EL. Commander and Executive Director of ERDC was COL James R. Rowan, EN. Director was Dr. James R. Houston. 


\section{Introduction}

The Environmental Security Technology Certification Program (ESTCP) funded the Naval Explosive Ordnance Disposal Technology Division (NAVEODTECHDIV) (lead agency), the U.S. Army Engineer Research and Development Center (ERDC), and the U.S. Army Environmental Center (AEC) to design and conduct controlled demonstrations of advanced unexploded ordnance (UXO) detection and discrimination technologies (Appendix A). The demonstrations were conducted at two prepared sites located on the island of Kaho'olawe in Hawaii (Figure 1) during the period 3 September through 30 November 2001 and were designed to evaluate the capabilities of state-of-theart technologies to detect, discriminate, and identify buried UXO in areas containing high concentrations of natural (magnetic rocks/soils) and man-made (munitions fragments) clutter. This report documents the results of these demonstrations and provides data to aid the Government in selecting effective and efficient systems for UXO detection and discrimination in difficult magnetic sites such as those encountered at Kaho'olawe Island.

\section{Background Information}

The Department of Defense (DoD) is currently involved in a number of UXO site remediation efforts where rapid transition of advanced technologies can potentially improve UXO detection efficiency, save substantial sums of money by reducing false alarms, and significantly expedite the transfer of lands for reuse. One of the most prominent of these efforts is the ongoing UXO cleanup of the Kaho'olawe bombing ranges. The major difficulty with this site is that the significant magnetic anomalies from geologic sources and near-surface metal fragments make traditional magnetometer-based surveys impractical. Active electromagnetic induction (EMI) sensors such as the Geonics EM-61 and the Geographical Technology Limited (GTL) TM-5 EMU are currently the primary sensors being used by the contractors at Kaho'olawe. Even though these EMI sensors have proven more effective at this site than passive magnetometers, their detection performance at Kaho'olawe sites has not been quantified, and they have been subject to very high false alarm rates. Parsons-UXB, the prime UXO contractor at Kaho'olawe, reports that as of 14 November 2001 they have detected 61,261 subsurface anomalies and, after digging, they have found that only 2.7 percent are UXO, 27 percent are false positives from geologic sources, and 70.3 percent are the result of buried metal from both UXO and non-UXOrelated materials. It should be noted that it is not possible to evaluate the detection performance (Probability of Detection or Pd) from these findings, since the 


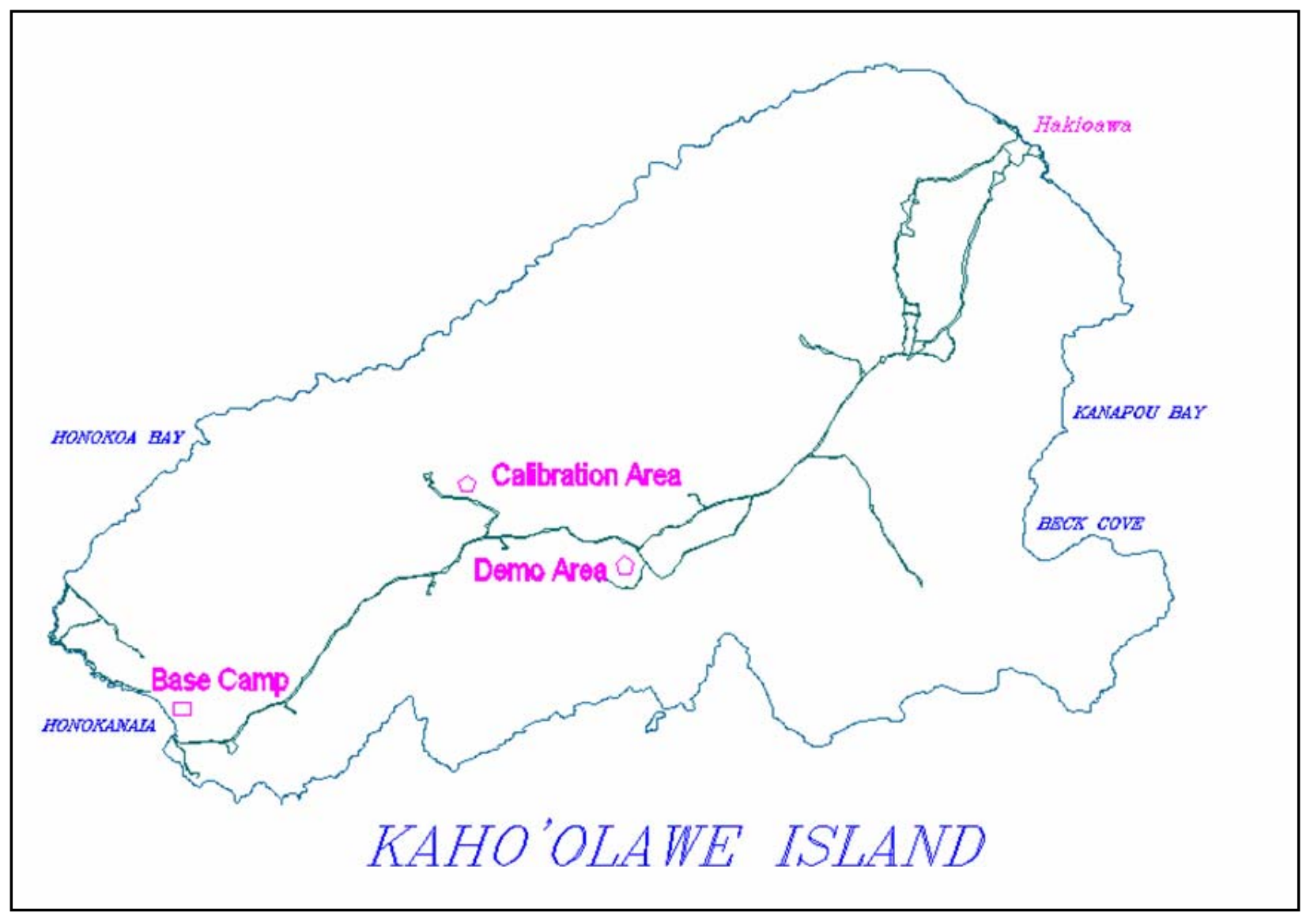

Figure 1. Overview of Kaho'olawe Island

actual number of buried UXO (groundtruth) is not known. ESTCP funded this project to address the critical need for more effective and efficient UXO technologies at sites such as Kaho'olawe.

The first phase of this ESTCP project was conducted at Jefferson Proving Ground (JPG), Indiana, during the period July through November 2000. This phase involved three advanced EMI sensing system demonstrators: (a) North American Exploration of Virginia, Inc. (NAEVA), Geophysics employing the Geonics EM-63 multi-channel, time-domain EMI system, (b) the Naval Research Lab (NRL) employing the single-channel, time-domain Electromagnetic Manportable System (EMMS), and (c) Geophex Ltd. employing the multifrequency, frequency-domain GEM-3 system. In addition, a commercial UXO surveying firm, Explosive Ordnance Disposal Technology, Inc. (EODT), was contracted to conduct standard "mag and flag" surveys of the JPG test areas in order to compare the EMI systems' performance with conventional techniques. The results of this first phase of demonstrations are documented by Cespedes (2001) and indicate that the EMI systems perform considerably better (higher detection rates, lower false alarms) than standard "mag and flag" surveys, especially in areas containing high levels of magnetic clutter from geologic sources. Since the first phase results provided strong indications that these technologies can significantly reduce false alarms resulting from high magnetic 
permeability in the soils and rocks, it was decided to evaluate the three systems under the more realistic and difficult geologic conditions found in Kaho'olawe.

In addition to the three advanced EMI systems demonstrated during the first phase, the ESTCP Program Office agreed to allow Geophysical Technology Limited (GTL) to demonstrate its advanced EMI sensor system, the TM-5 Electromagnetic Unit (EMU), as part of the second phase tests at Kaho'olawe. GTL provided its own funding to participate in this phase, and ESTCP agreed to fund the additional costs associated with monitoring the GTL field surveys and evaluating their performance. It should be noted that GTL has participated in previous demonstrations conducted at JPG and was among the top performers in several of the test scenarios including the small UXO sites (e.g., grenades and submunitions) (U.S. Army Environmental Center (USAEC) and

NAVEODTECHDIV 1997). In addition, GTL has considerable operational experience with the TM-5 EMU in Kaho'olawe live sites.

For baseline comparisons with technologies currently used at Kaho'olawe sites, Parsons-UXB conducted standard EM-61 digital surveys as well as "EM and Flag" surveys using the EM-61 and the TM-5 EMU in a real-time detection/discrimination mode.

The focus of this demonstration project was to evaluate these advanced EMI technologies under realistic and difficult field conditions in order to quantify their detection, discrimination, cost, and production rates while operating at several areas within Kaho'olawe with varying degrees of target/clutter densities and magnetic noise levels. The purpose of this report is to aid managers of UXO cleanup projects as well as regulators and other stakeholders to make informed decisions concerning the capabilities, costs, and risks associated with applying these technologies to their site-specific UXO remediation problems.

\section{Official DoD Requirement Statement(s)}

This project addresses the Tri-Service Environmental Quality Research, Development, Test, and Evaluation Strategic Plan, UXO requirements, and more specifically, the U.S. Army requirement A(1.6a), titled: Unexploded Ordnance (UXO) Screening, Detection, and Discrimination. Additionally, the project describes the FY99 Army Environmental Requirements and Technology Assessments (AERTA). This Army requirement has been ranked as the highest priority user need in the Environmental Cleanup Pillar. In addition, this project addresses the UXO detection and discrimination requirements and recommendations described in the Defense Science Board Task Force Final Report on UXO Clearance and Remediation published in 1998 and will provide data to support the development of more accurate estimates of the overall DoD UXO environmental remediation costs.

The advanced technologies demonstrated as part of this effort address all aspects of the requirements for land-based, man-portable buried UXO detection and discrimination systems. The results of these demonstrations will be used to quantify the capability of state-of-the-art systems to detect, discriminate, locate, 
and identify buried targets. The performance of the advanced systems was compared with the baseline capability demonstrated by the onsite contractor, Parsons-UXB.

This technology demonstration creates a framework for the evaluation of state-of-the art sensor technologies to detect, locate, and identify UXO. Baseline technology performance is established, and technology capabilities and limitations are assessed. Results from this program will be widely distributed to aid in the selection and utilization of sensors and data analysis techniques for UXO characterization and restoration efforts.

\section{Objectives of the Demonstration}

The primary technical objective of this demonstration project was to evaluate the detection and discrimination capabilities (including production rates and costs) of advanced UXO systems in difficult magnetic clutter environments such as those encountered at Kaho'olawe. For this phase of the project, one $90-\mathrm{m}$ by 111.1-m (1-hectare) area and 10 (not necessarily contiguous) $30-\mathrm{m}$ by $30-\mathrm{m}$ test grids within the Kaho'olawe Quality Assurance (QA) Range were prepared to present a limited range of target/clutter/topography/vegetation/magnetic background conditions to the various demonstrators.

The evaluation objectives for this phase of demonstrations were as follows:

a. To evaluate the demonstrators' detection and discrimination capabilities by means of surveys of ten $30-\mathrm{m}$ by $30-\mathrm{m}$ grids and one 1-hectare area within the Kaho'olawe QA Range under realistic target/ geologic clutter/ man-made clutter/topography scenarios while operating as efficiently as possible (minimizing time, manpower, and costs).

$b$. To evaluate the demonstrators' ability to analyze survey data in a timely manner and provide prioritized "dig lists" with associated confidence levels.

c. To collect data on manpower and time required to collect field data necessary to produce their final products (prioritized dig sheets and georeferenced anomaly maps).

$d$. To compare the performance of the advanced systems with the baseline technologies that are currently employed at Kaho'olawe.

e. To provide high quality, well ground-truthed, georeferenced data for postdemonstration analysis and development of Receiver Operating Characteristic (ROC) curves.

\section{Regulatory Issues}

The principal regulatory issue affecting UXO detection and discrimination technologies is gaining confidence and approval from Federal, state, and local regulators, stakeholders, and users. In addition, acceptance of these innovative 
technologies from agencies such as the U.S. Army Corps of Engineers and the Naval Facilities and Engineering Command is needed to ensure that future Requests for Proposals (RFPs) for UXO cleanup projects will be written in a manner that will either sanction these technologies, or at least allow their inclusion in proposals for site work. Members of the regulatory community who are aware of these technology demonstrations are listed in Appendix A.

\section{Previous Testing of the Technology}

Versions of the technologies demonstrated under this effort have been previously tested as part of other DoD and Army sponsored demonstrations including the Defense Advanced Research Projects Agency (DARPA) Clutter Experiment (FY97), the Jefferson Proving Ground Phases II through IV Demonstrations, and a number of ESTCP-funded field demonstration projects. However, this ESTCP project represents the first set of controlled field experiments at an actual remediation site where these advanced technologies have been tested under realistic conditions that allowed for side-by-side comparison of detection/discrimination performance, production rates, and costs. 


\section{Technology Description}

\section{Description}

The five electromagnetic induction sensing systems that participated in this demonstration project at Kaho'olawe included the following (in chronological order):

a. Geonics Ltd. EM-63, a multichannel time domain EMI sensor operated by personnel from NAEVA Geophysics

b. GTL TM-5 EMU, a multiperiod time-domain EMI sensor operated by GTL and Parsons-UXB Technology personnel

c. Geophex Ltd. GEM-3, a multichannel frequency-domain EMI sensor system operated by Geophex Ltd. Personnel

d. NRL Man-Portable EM System (EMMS) adjunct to the MTADS system, a single-channel time-domain EMI sensor operated by personnel from NRL with processing support from AETC Corp.

e. Geonics EM-61, a single channel time domain EMI system operated by Parsons-UXB.

Each of the five sensors was integrated into a man-portable platform that included data acquisition/storage that merged the sensor data with position data collected by differential Global Positioning System (GPS) receivers. In addition, Parsons Technology operated the TM-5 EMU and the EM-61 in a field discrimination ("EM and Flag") mode. In this mode, the systems did not record digital sensor data, and the only permanent record consists of the identified UXO locations that were marked by a separate GPS survey crew. These sensor systems are shown in Figures 2 through 7 conducting surveys at the Kaho'olawe QA range. Detailed descriptions of these sensing systems are included in studies by Geonics Limited (1999 and 2000), GTL (2002), Won et al. (1998), and NRL (2001).

\section{Strengths, Advantages, and Weaknesses}

The following paragraphs represent a summary of the perceived, claimed, and documented capabilities of each of the sensors employed by the technology demonstrators. 


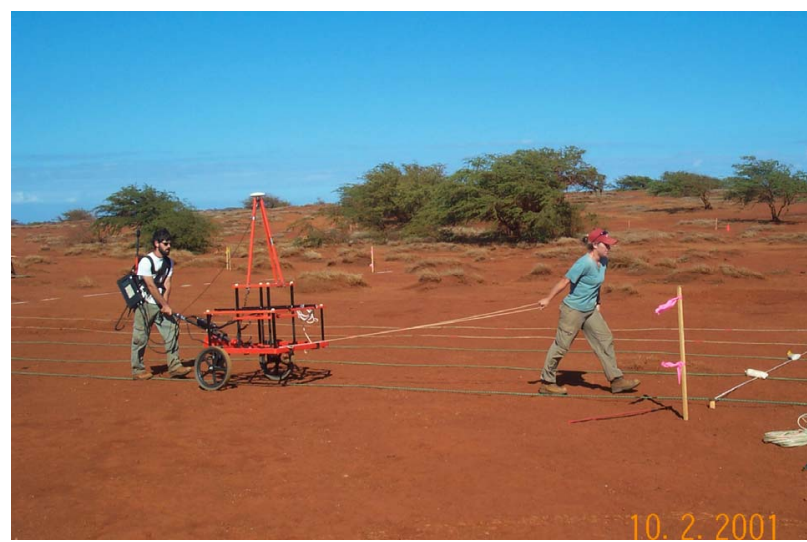

Figure 2. EM-63 operated by NAEVA

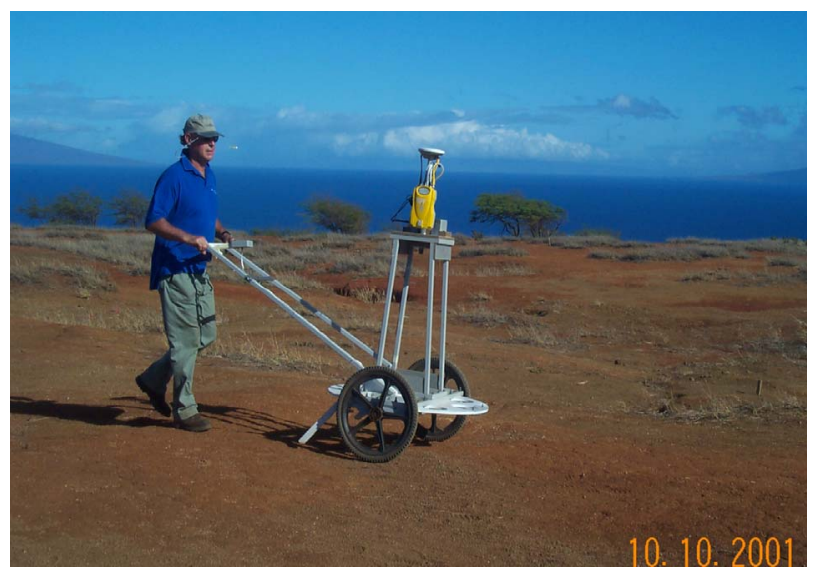

Figure 4. GEM-3 operated by Geophex, Ltd.

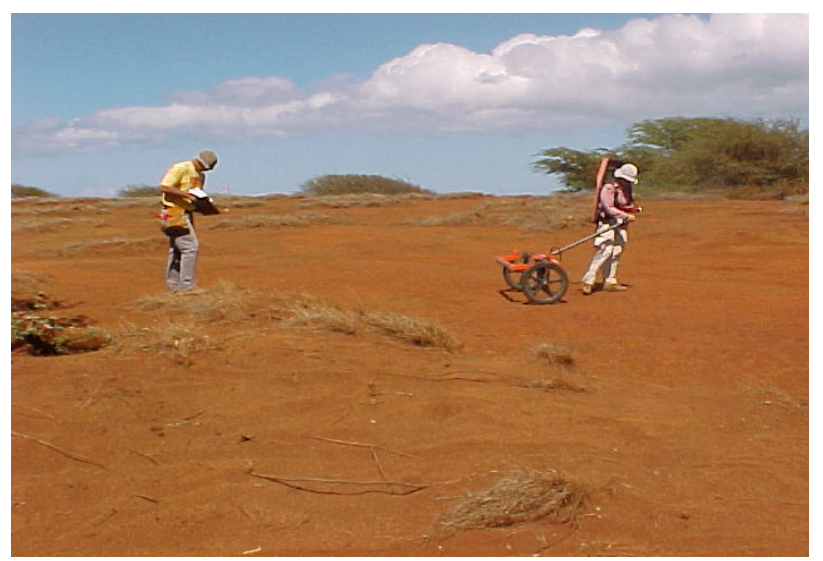

Figure 6. EM-61 operated by Parsons

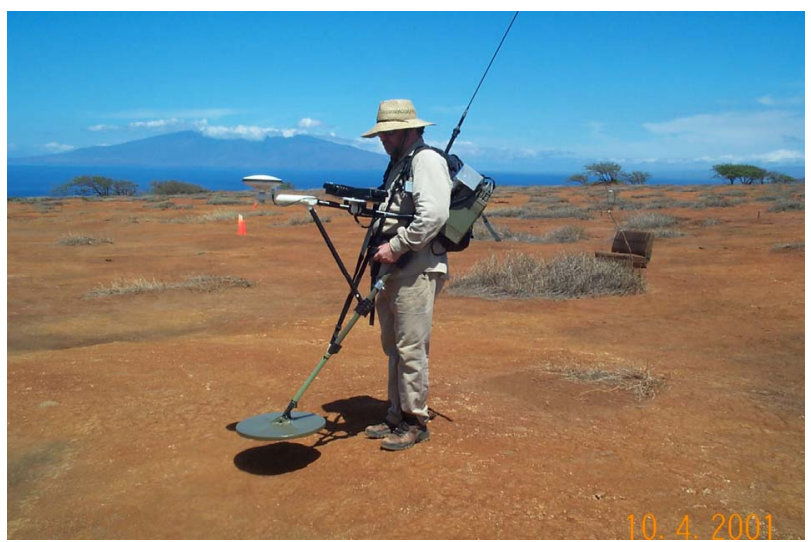

Figure 3. TM-5 EMU operated by GTL

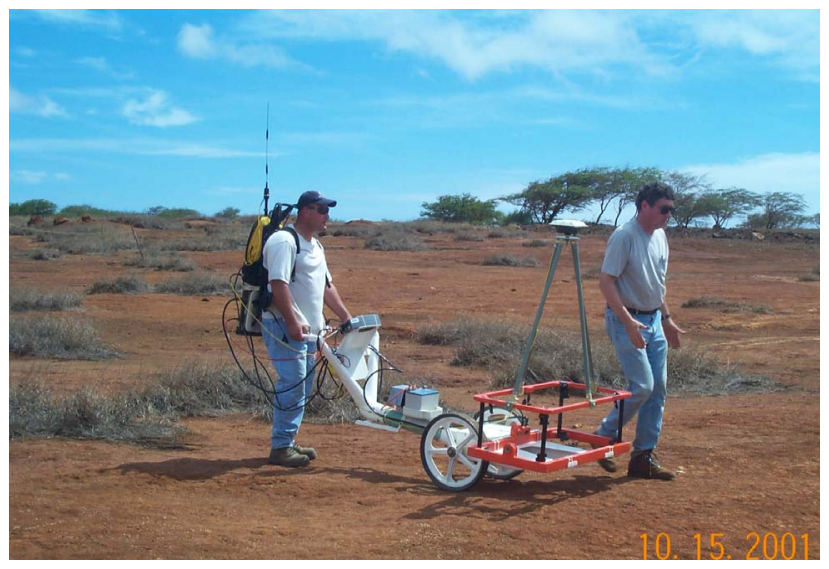

Figure 5. EMMS operated by NRL

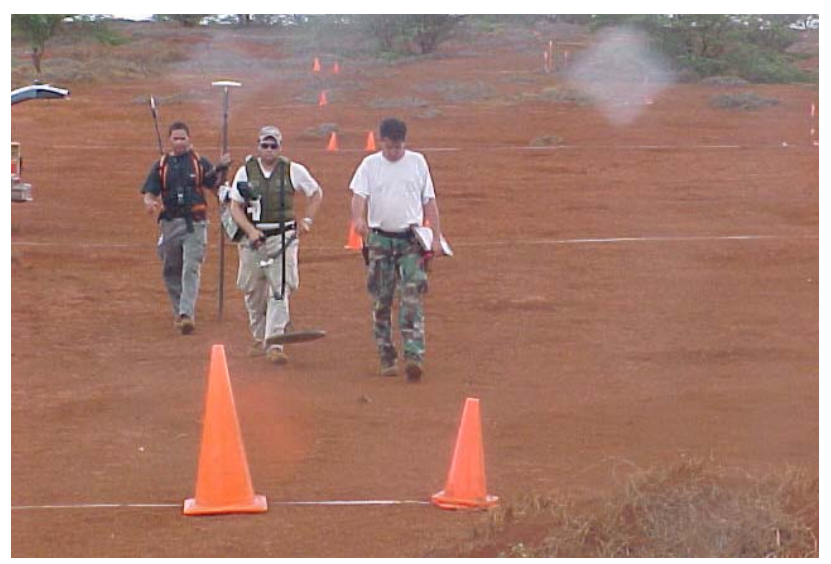

Figure 7. TM-5 EMU operated by Parsons 


\section{Geonics EM-63}

The claimed strengths of the EM-63 lie in its ability to rapidly collect multiple channels of time-domain information at each survey point. The EM-63 collects up to 26 geometrically spaced time gates covering the time decay response in the range from $180 \mu \mathrm{sec}$ to $25 \mathrm{msec}$ after pulsing of the transmitter coil (Geonics Limited 1999). Since the shape of the decay curve is dependent on the shape, size, orientation, and metal composition of the buried object, it is hypothesized that the EM-63 data channels can provide the information required to detect, discriminate, and identify the buried UXO targets, and to significantly reject responses from geologic materials and metallic clutter. The EM-63 is a commercially available sensor (produced by Geonics Ltd. which also manufactures the EM-61) and has been ruggedized for field use. Another significant strength of the system demonstrated at JPG is the processing expertise of NAEVA personnel. During previous JPG demonstrations, NAEVA has consistently ranked among the top performers, even though they had employed sensor data like other less-successful demonstrators. Perceived weakness of the NAEVA EM-63 demonstration system was that NAEVA had limited experience with the EM-63, since it has only recently become available and has undergone a number of hardware and software modifications. In addition, the analysis techniques were still under development and had not been fully tested in difficult conditions such as those encountered in Kaho'olawe.

\section{GTL TM-5 EMU}

The GTL TM-5 EMU is a multiperiod, time-domain EMI system integrated with real-time processing that is claimed to provide automatic background leveling capability for enhanced detection and discrimination capabilities in sites containing high levels of magnetic interference (GTL 2002). Unlike the other EMI systems tested during this demonstration, the TM-5 is a monocoil sensor with one element acting as both transmitter and receiver. The TM-5's transmitted waveform is referred to as "multiperiod" because it consists of a wavetrain with a single, longer pulse followed by three shorter pulses with the same length, all of which are repeated at a rate of approximately $1,200 \mathrm{~Hz}$. The long pulse is four times wider than each of the short pulses. Each decay period following each of the four pulses is sampled twice, with the specific details of gate timing, periods, and the method of combining them for analysis being proprietary information of MineLab Electronics and not available for publication. GTL has taken the sensor and electronics provided by MineLab and integrated advanced processing and positioning information to produce the TM-5 EMU specifically for UXO detection applications. The TM-5 EMU can perform UXO discrimination in real time, or the data can be recorded in digital form and postprocessed to allow for more in-depth analysis and interpretation. Unfortunately, descriptions of the real-time and postprocessing techniques are also proprietary to GTL and not releasable. In spite of GTL's assurance that these details would be fully disclosed in return for the Government's agreeing to allow their participation in the Kaho'olawe demonstrations, such information has not been provided. Thus, claims as to the TM-5 EMU's automatic background leveling capabilities and its superior ability to operate in highly conductive and/or magnetic environments cannot be fully evaluated. 


\section{Geophex GEM-3}

The strength of the GEM-3 system is claimed to lie in its ability to rapidly collect multiple channels of complex frequency domain EMI data over a wide range of audio frequencies $(30 \mathrm{~Hz}$ to over $20 \mathrm{kHz})$. This allows for performing what Geophex Ltd., the developer of the system, calls Electromagnetic Induction Spectroscopy (EMIS) on buried objects (Won et al. 1998). EMIS provides a method to discriminate UXO targets from natural and manmade clutter objects by means of their unique, complex (inphase and quadrature) frequency responses. The GEM-3 system was the top performer in the discrimination and identification tests conducted during JPG Phase IV. A concern and possible weakness of the GEM-3 is that, to accomplish a wide-area detection and discrimination survey in a reasonable time, it must keep the number of frequencies to a relatively low number (seven in the case of these Kaho'olawe tests) and must transmit them simultaneously rather than sequentially as was done during the static JPG IV tests. This simultaneous transmission of multiple frequencies may reduce the power dedicated to each frequency, and this could affect the depth capability of the GEM-3 system. Another possible weakness of the GEM-3 system is that high-accuracy position information is required to perform the discrimination. In the previous JPG IV demonstrations, this high degree of position accuracy was obtained by means of templates placed over specified target locations allowing static point measurements to be made. That approach was not viable for the widearea search requirements of the current project, and Geophex had to rely on GPS position information, which results in significantly greater position errors and sparser data sets. Finally, it was observed that the GEM-3 system is still undergoing development, and the sensor design, platform, data acquisition system, and analysis approaches have not been finalized or optimized.

\section{NRL EMMS}

The EMMS is derived from the highly successful Multi-Sensor Towed Array Detection System (MTADS) development effort and thus incorporates many of its sensing, navigation, and data analysis system (DAS) advances demonstrated and documented in a number of ESTCP-funded field demonstrations. The specifications and performance improvements incorporated into the version of the EMMS demonstrated at Kaho'olawe are fully described in the ESTCP report titled "Man-Portable Adjuncts for the MTADS" (NRL 2001). Based on a modified version of the commercially available Geonics EM-61 (with the $0.5-\mathrm{m}$ by $1.0-\mathrm{m}$ transmitter coil), the most widely used EMI system for UXO detection applications, the EMMS sensor is expected to have good UXO detection capability to the maximum depths of the objects emplaced at Kaho'olawe. Coupled with the very high accuracy of the MTADS-derived, digital inclinometer/GPS system, the EMMS is expected to produce high quality georeferenced EMI data. A potential limitation of the EMMS is the single channel of data available, which may limit the discrimination performance compared to what can ultimately be achieved by multichannel systems. 


\section{Geonics EM-61}

The Geonics EM-61 system is a single-channel time-domain metal detector. It is the most mature and widely applied EMI technology for UXO detection surveys (Geonics Limited). The system is available with different coil configurations and the one used at Kaho'olawe by Parsons-UXB incorporated the large $1-\mathrm{m}$ by $1-\mathrm{m}$ coils. As is the case with other time-domain systems, the transmitter coil generates a pulsed primary magnetic field in the earth, which induces eddy currents in nearby metallic objects. The eddy current decay produces a secondary magnetic field measured by the receiver coil. By taking the measurement at a relatively long time after the start of the decay, the designers of the EM-61 predict that the currents induced in the ground have fully dissipated and only the current in buried metal objects is still producing a secondary field. The responses are recorded and displayed by an integrated data logger. Another advantage of the EM-61 system's reputation as the EMI system of choice at UXO sites is that it is supported by a number of software development firms. For example, Geosoft, one of the leading designers of software for geophysical applications, produces a version of its UX-Detect software specifically tailored to accept and analyze EM-61 data. Similar to the EMMS described above, the primary perceived weakness of the EM-61 system is that its single-channel time decay window does not provide adequate information to discriminate buried UXO from other conductive or highly permeable buried objects (both manmade and naturally occurring).

\section{Factors Influencing Cost and Performance}

Data on the various factors that influence the overall cost and performance of each of these systems in actual UXO remediation efforts were collected as part of this field demonstration effort, and include the following:

a. Equipment setup, calibration time, and man-hour requirements.

$b$. Time and man-hour requirements to survey the demonstration test areas.

c. Downtime resulting from system malfunctions and maintenance requirements.

d. Reacquisition/resurvey time and man-hour requirements.

e. Accuracy of georeferenced maps and prioritized dig lists with respect to:

(1) Probability of Detection (P(det)).

(2) False alarm rates (Pfp, FAR, Total FAR).

(3) Discrimination capability $(\mathrm{P}($ disc $))$.

(4) Identification capability.

(5) Target location accuracy.

The Demonstration Workplan (Naval Explosive Ordnance Disposal Technology Division (NAVEODTECHDIV) 2001a) and Chapter 4 of this report 
include detailed descriptions of the methods and metrics used to evaluate each of the cost and performance factors. 


\section{Site/Facility Description}

\section{Background}

Site selection criteria, site description, and site preparation activities are described in detail in the Site Preparation Plan (NAVEODTECHDIV 2001b), but short descriptions of the island, the calibration and demonstration sites, and the emplaced targets are presented in this section.

Kaho'olawe Island consists of the summit of a single volcanic dome that reaches a peak elevation of $450 \mathrm{~m}(1,477 \mathrm{ft})$ above mean sea level (Lua Makika point at the northeastern part of the island). It is one of the oldest of the main group of Hawaiian Islands and is separated from Maui by the 11-km (6.9-mile) wide Alalake'ike Channel and from Lana'i by the $28 \mathrm{~km}$ (17.5 mile)

Kealaikahiki Channel. It is $18 \mathrm{k}$ (11 miles) long and $10 \mathrm{~km}$ (6 miles) wide with an area of 28,776 acres. Kaho'olawe Island was used as a weapons range and military training area from 1941 until 1990. Title X of the FY1994 Department of Defense Appropriations Act was enacted in November 1993 and directed the cleanup of ranges in Kaho'olawe Island. Title X allocated $\$ 400 \mathrm{M}$ for UXO remediation starting in 1993 and required that Kaho'olawe be transferred to a Native Hawaiian sovereign entity no later than November 2003.

The island's geology consists predominantly of basalt, hardpan, and sand. The magnetite-containing basaltic rocks and soils have precluded the use of magnetometers for UXO detection and have been the source of a significant number of false alarms encountered by the EMI sensors currently used by the UXO remediation contractors. Kaho'olawe's surface features consist primarily of dry land vegetation and hardpan (Figures 8 and 9). The island's climate is windy and very dry, averaging only 25.4 to $50.8 \mathrm{~cm}$ (10 to $20 \mathrm{in}$.) of rainfall per year (mostly on the eastern side of the island). The island has had a history of overgrazing by sheep and cattle, destruction of vegetation by goats, deforestation by settlers, and finally, damage caused by target bombing and shelling by the United States Military.

\section{Site/Facility Characteristics}

The previously established Kaho'olawe UXO Remediation Project's Quality Control (QC) and Quality Assurance (QA) ranges, shown in Figure 10, were selected for this project's calibration (Figure 11) and demonstration areas, respectively. The calibration and demonstration sites were surveyed using 


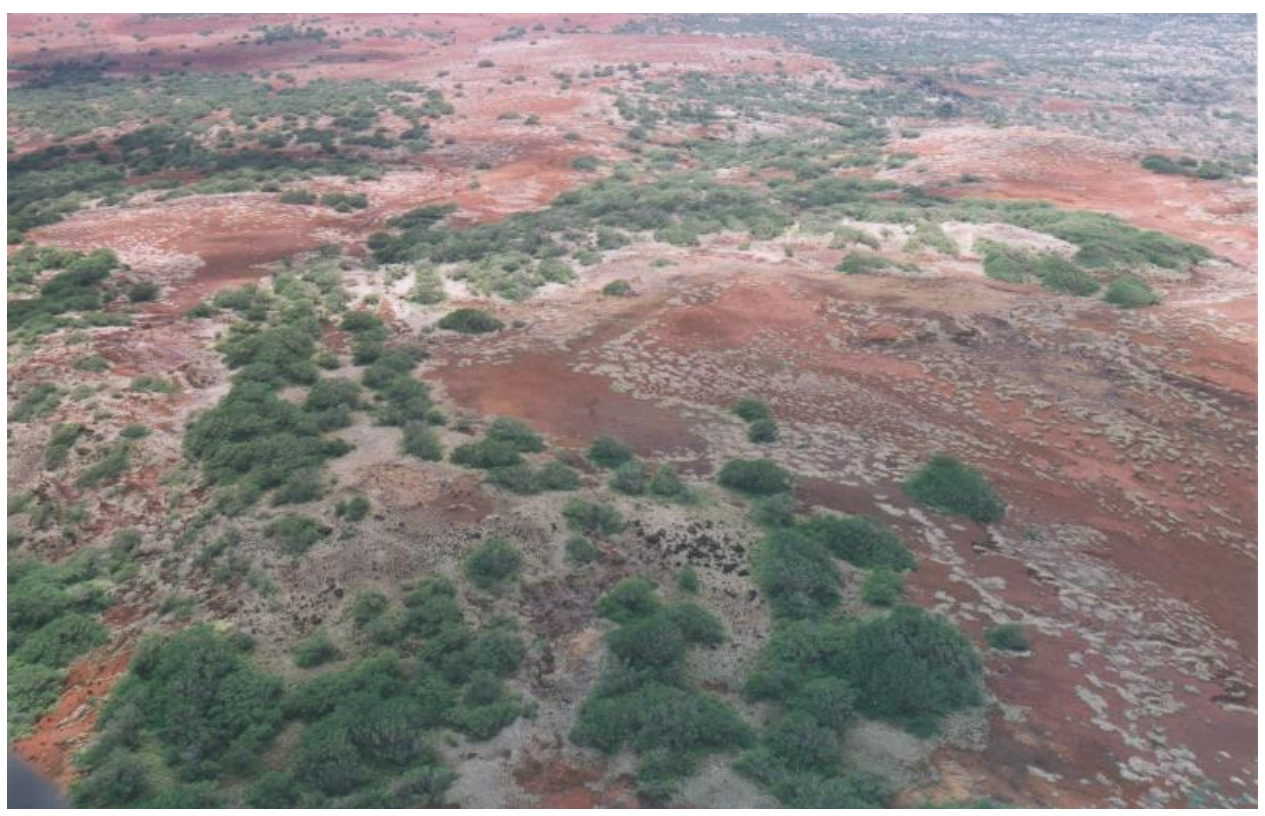

Figure 8. Overview of Kaho'olawe showing erosion of terrain

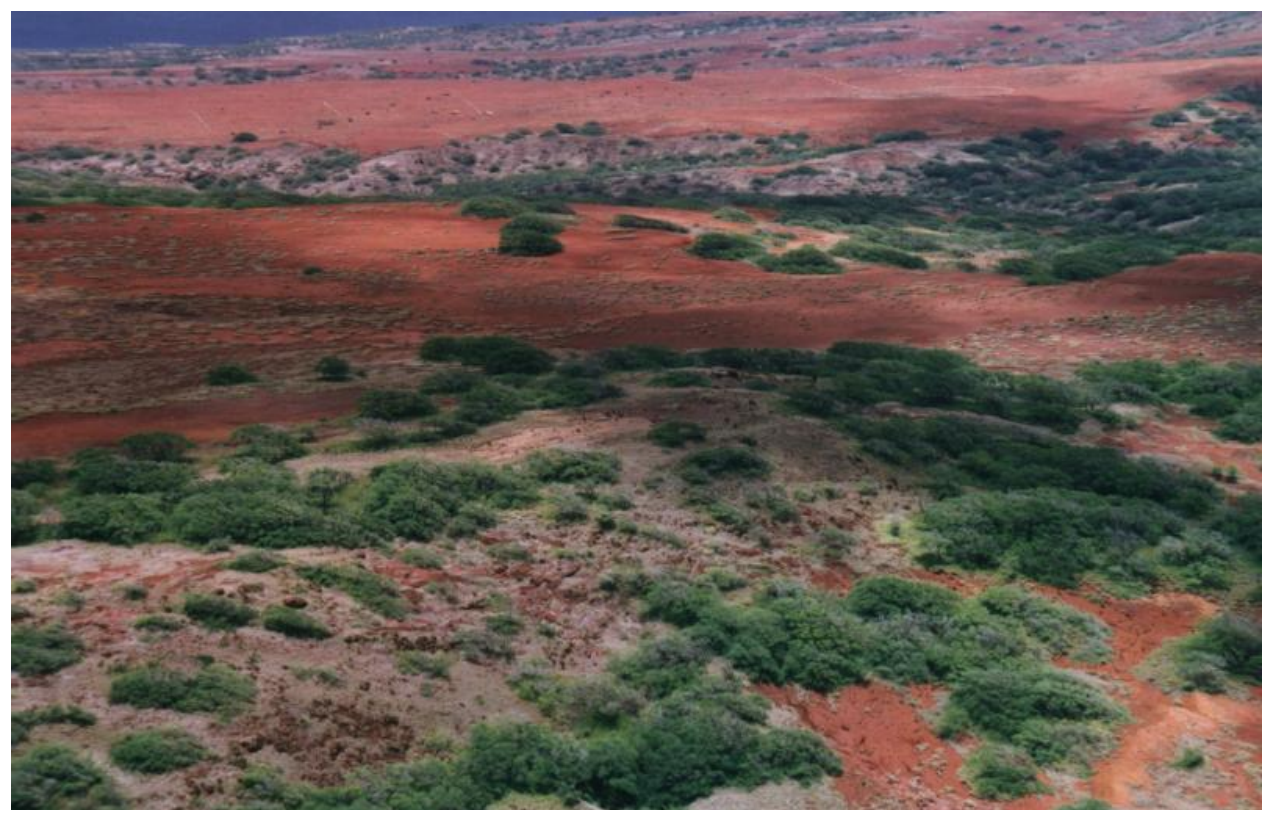

Figure 9. Overview of Kaho'olawe vegetation and hardpan terrain

electronic theodolites (Leica Model TCA 1102) and real-time kinematic differential global positioning system (RTK-DGPS) survey equipment. Both sites were oriented to true north and each corner of each range was staked with a ferrous rod and its coordinates recorded. The magnetic variation at the Kaho'olawe site is $9^{\circ}-59^{\prime}$ East. Monuments near the calibration and demonstration areas were brought up to first-order accuracy during the initial site preparation activities, and updated coordinates were provided to the demonstrators prior to the scheduled demonstrations. 


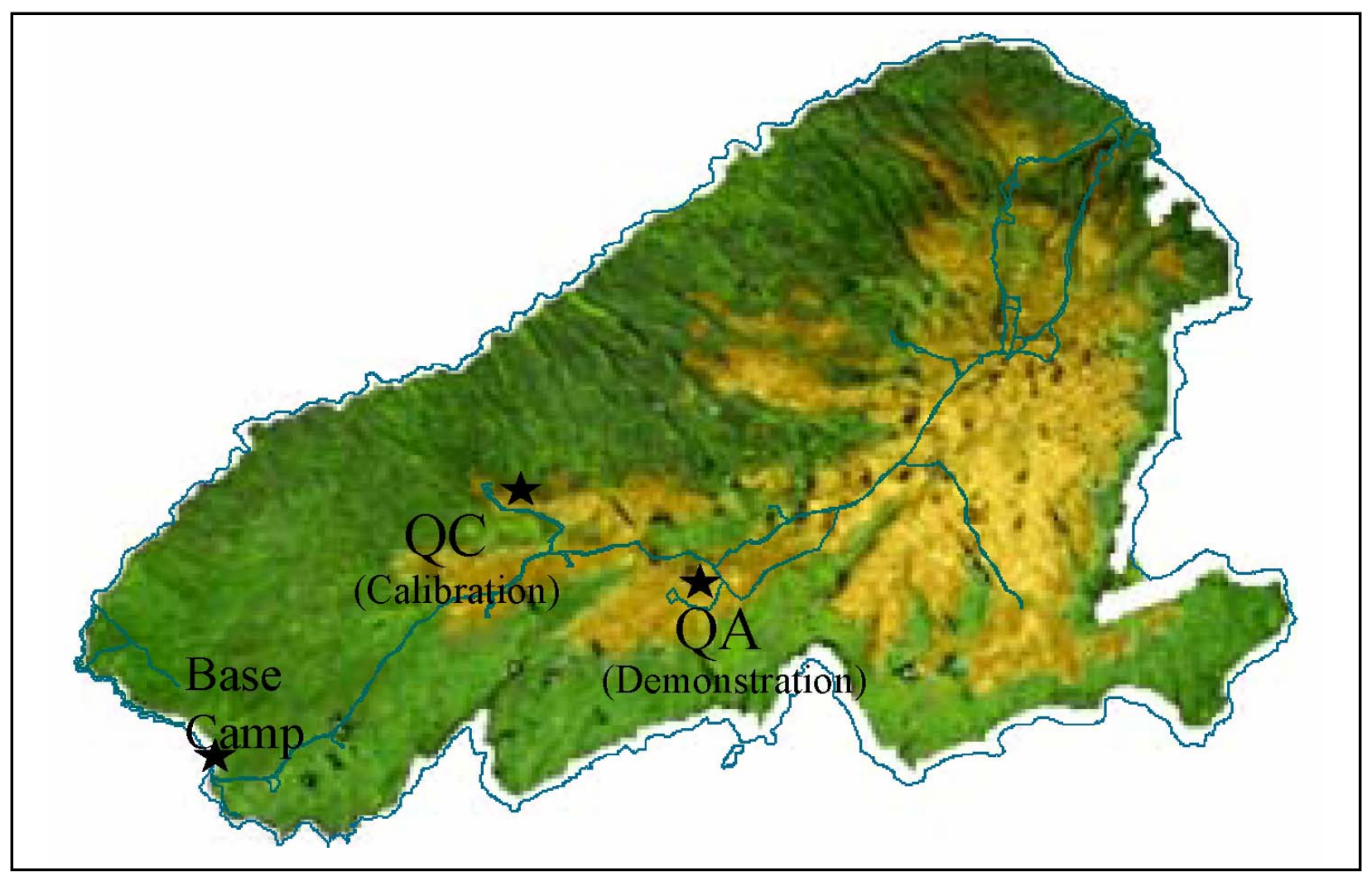

Figure 10. Topography map with outline of Base Camp, calibration, and demonstration ranges

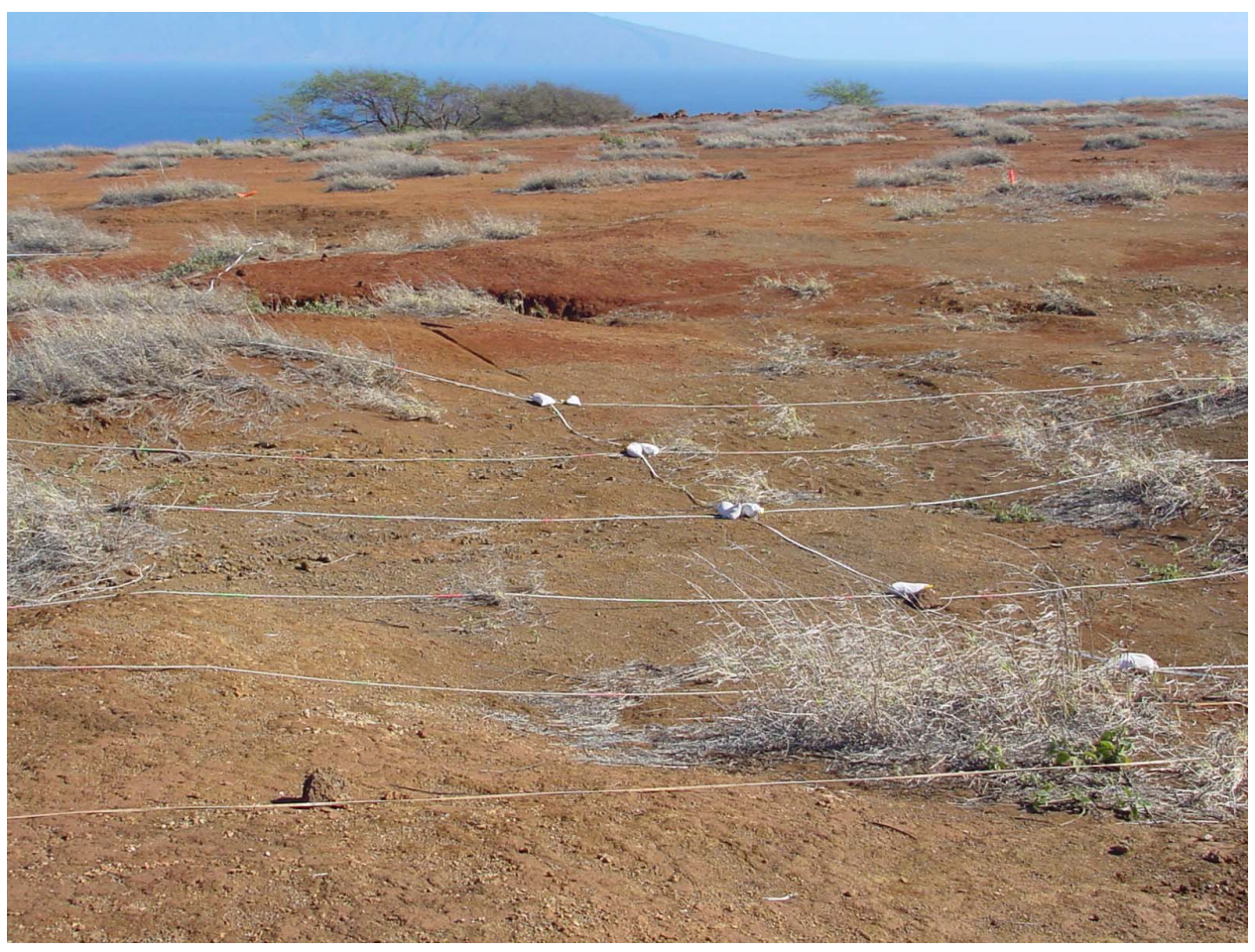

Figure 11. Calibration area within the QC range 


\section{Calibration area}

The calibration area consisted of three $30-\mathrm{m}$ by 30 -m grids and was established to allow the demonstrators to conduct system calibration, signature collection, and algorithm development prior to participating in the blind tests conducted in the demonstration areas. The complete groundtruth of all items emplaced in the calibration area was made available to each demonstrator prior to arrival on Kaho'olawe.

The demonstration area was subdivided into three areas: Area A consisted of four $30-\mathrm{m}$ by $30-\mathrm{m}$ grids, Area B consisted of nine $30-\mathrm{m}$ by $30-\mathrm{m}$ grids and three partial grids (totaling 1 hectare), and Area $\mathrm{C}$ consisted of six $30-\mathrm{m}$ by $30-\mathrm{m}$ grids (Figure 12). Figure 13 shows the QA range looking from grid 5B to grid 1E.

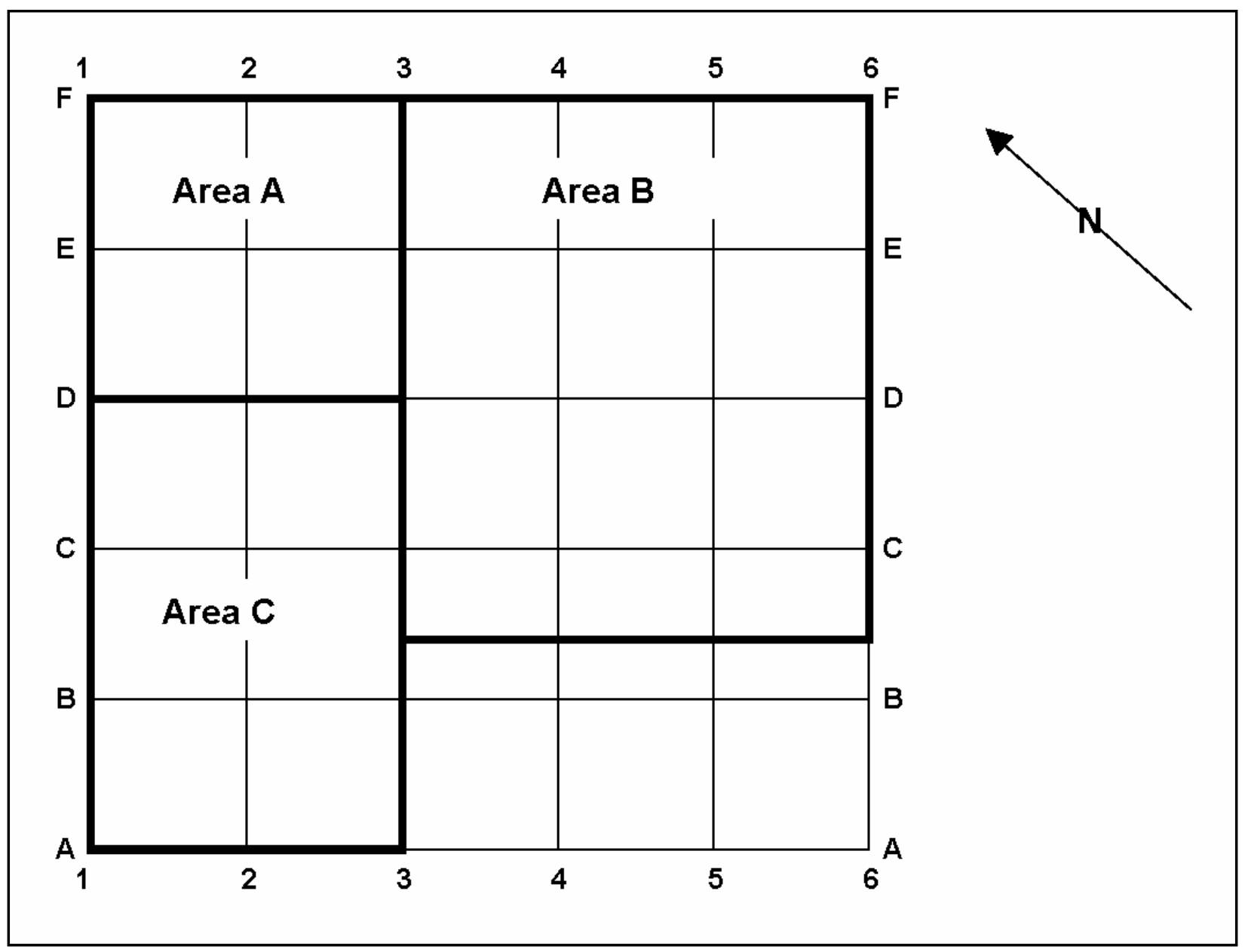

Figure 12. Layout of demonstration areas 


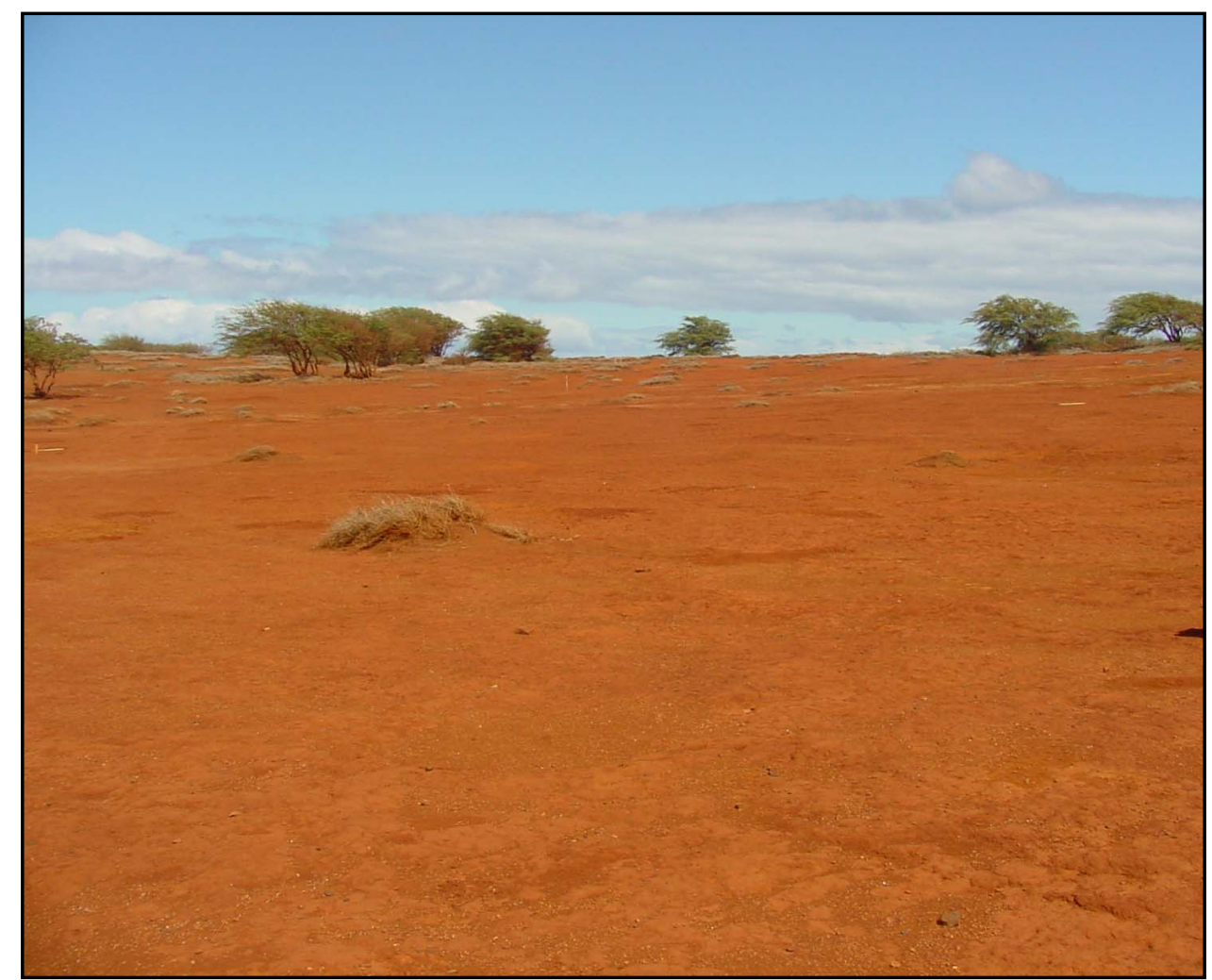

Figure 13. Photo of Demonstration area within the QA range

\section{Target emplacement}

The target list consisted of the following 18 items (items in bold were taken from the Standard Test Sites Program stockpile and the rest are from Kaho'olawe UXO cleanup operations):

- 20-millimeter $(\mathrm{mm})$ projectile

- 40-mm projectile

- 60-mm mortar

- 81-mm mortar

- 2.25-in. rocket

- 2.75-in. rocket warhead

- 3-in. projectile

- 5-in. projectile

- $\quad$ 105-mm projectile

- SMAW rocket

- LAAW 
- BDU-3 practice bomb

- BDU-33 practice bomb

- MK-82 practice bomb

- MK-3 practice bomb

- MK-81 practice bomb

- MK-106 practice bomb

- MK-83 practice bomb

All unfired, inert UXO items were thoroughly degaussed either at NAVEODTECHDIV, at Aberdeen Proving Ground, Maryland, or in the field prior to emplacement in order to remove any remnant magnetic moment. Each degaussed item was checked for remnant magnetization using a G- 858 total field magnetometer. Target and clutter items were weighed, measured, and photographed, and the excavation crew emplaced the preselected target/clutter item at the location, orientation, and approximate burial depth specified in the Site Preparation Plan.

Prior to emplacing any targets, all UXO target emplacement locations (larger than $20 \mathrm{~mm}$ ) were surveyed with a Geonics EM-61 HH and a Geometrics G-858 in order to record background noise prior to emplacement and also to allow removal of any metal objects in the vicinity (within a 2-m by 2-m area) of the target location. In addition, one $30-\mathrm{m}$ by $30-\mathrm{m}$ grid within the Demonstration area that contained no preemplaced items was surveyed with both the EM-61 and the G-858. These preemplacement surveys are included in the data archive described in Appendix B. Figures 14 and 15 show overviews of the clutter and target items in the calibration and demonstration areas, respectively. Detailed information on these items is included in the Site Preparation Plan. 


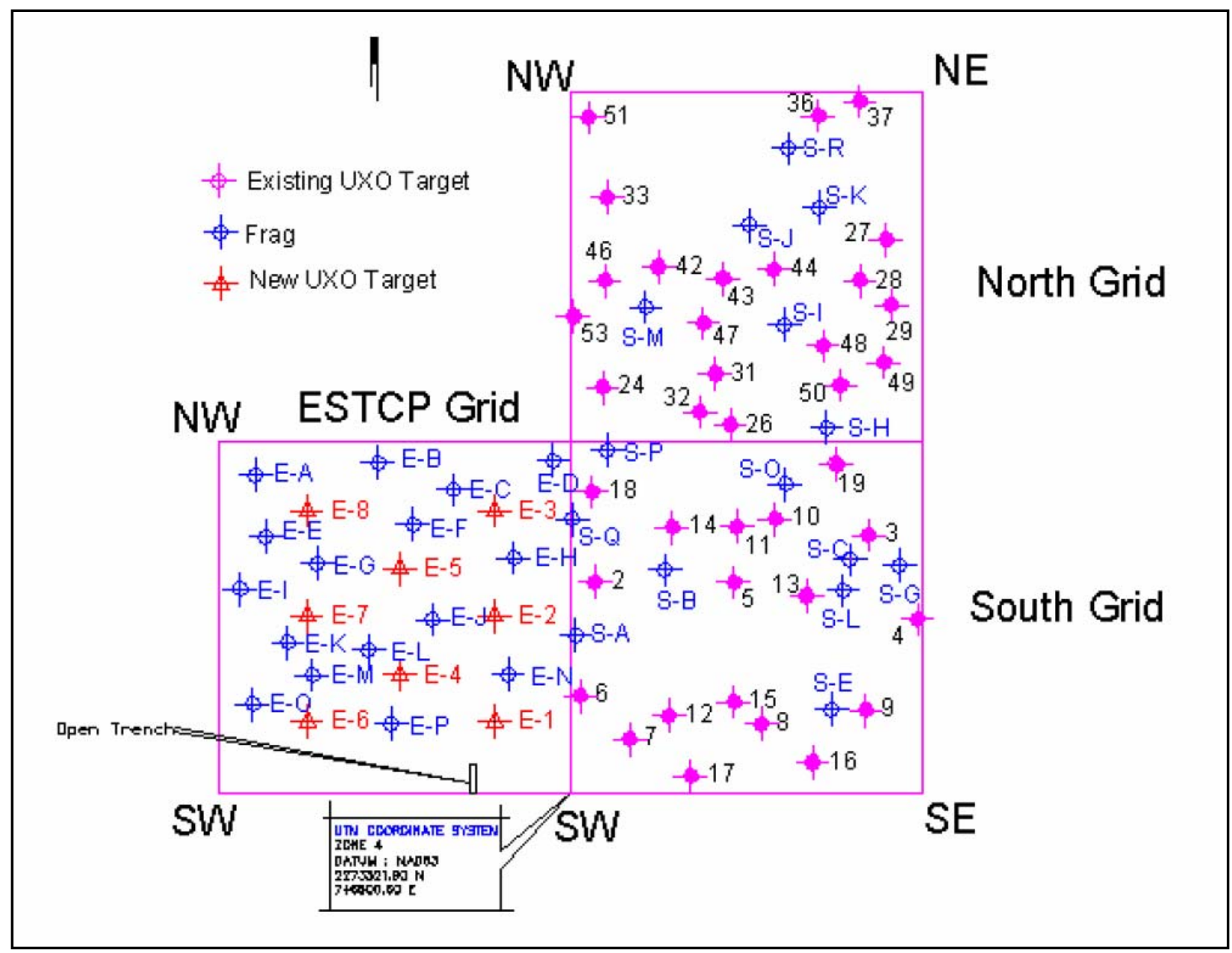

Figure 14. Kaho'olawe calibration area target emplacement plan 


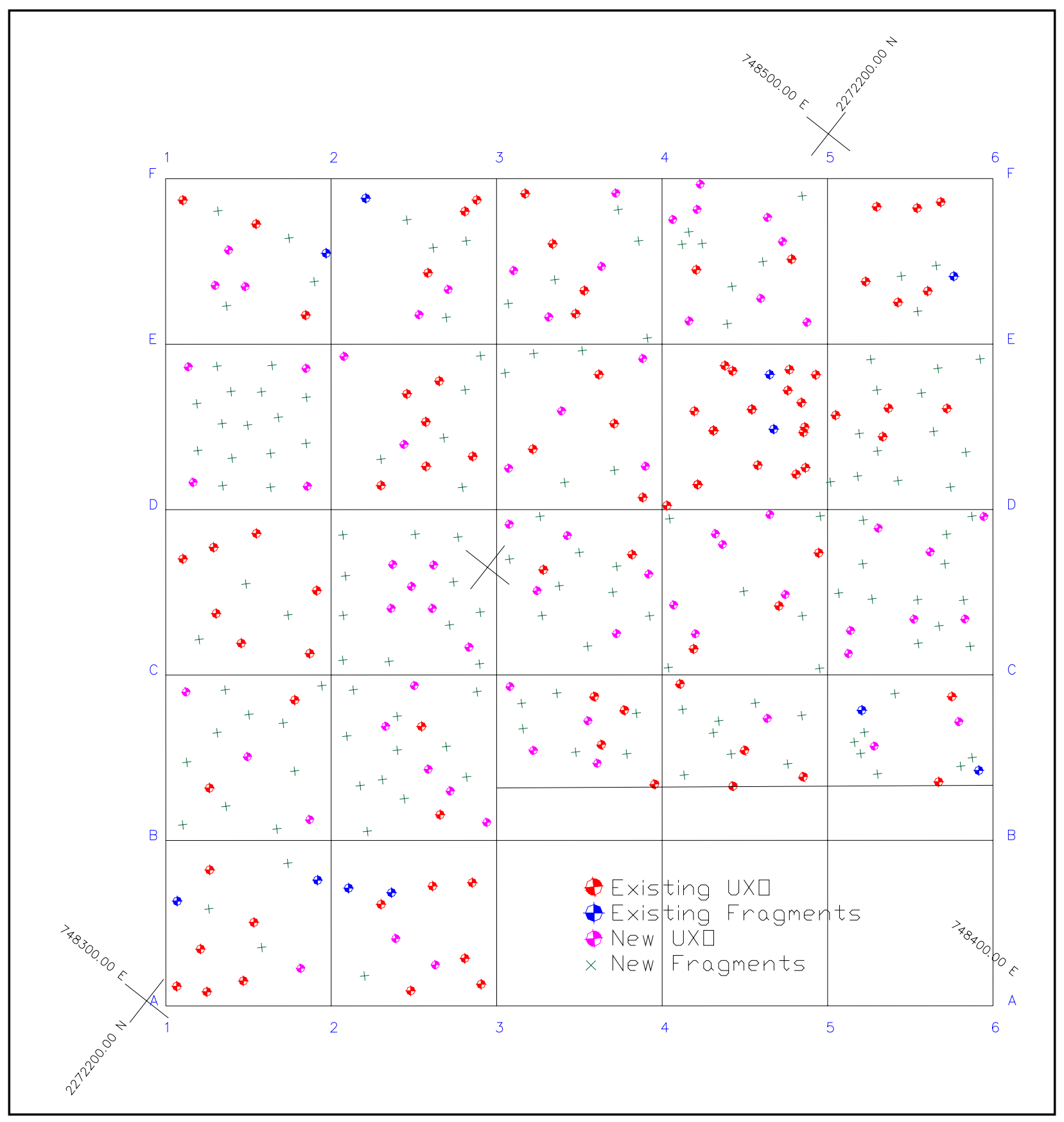

Figure 15. Kaho'olawe site map showing emplaced items in Areas A, B, and C of the demonstration site 


\section{Demonstration Approach}

\section{Performance Objectives}

The objective of this demonstration was to evaluate the performance of advanced EMI technologies in very difficult UXO target, clutter, and geologic noise scenarios such as those commonly encountered in Kaho'olawe UXO remediation efforts. As was the case at JPG during the first phase of this project, this demonstration attempted to evaluate the detection, discrimination, and identification capabilities of advanced UXO systems while simultaneously evaluating their production rates, manpower requirements, and costs.

The scope of this effort was to collect sufficient information from a limited range of test scenarios in order to quantify the advantages and disadvantages of each of the four EMI technologies so that they may be properly applied to specific UXO cleanup problems. The immediate goal of this effort is to quantify any performance and cost improvements resulting from the application of advanced EMI technologies so that they can be rapidly transitioned to Kaho'olawe-type operations where natural (magnetic rocks/soils) and man-made (munitions fragments) clutter have rendered conventional UXO cleanup efforts both expensive and ineffective. A longer-term objective of this demonstration is to collect and archive high-quality, georeferenced data to support future sensor development and improvements in UXO analysis technologies.

The performance objectives for this demonstration are as follows:

a. To conduct surveys of one 1-hectare area and ten $30-\mathrm{m}$ by $30-\mathrm{m}$ areas within Kaho'olawe Island under very difficult but realistic target/ geologic clutter/ man-made clutter scenarios while operating as efficiently as possible (minimizing time, manpower, and costs).

$b$. To analyze survey data in a timely manner (prior to departing Hawaii) and provide "dig lists" that include detection, discrimination, and identification estimates with associated confidence levels as well as georeferenced anomaly maps. Note that unlike the first phase demonstrations at JPG, this demonstration phase did not require that the processing be performed onsite.

c. To detect and locate all buried ordnance while minimizing false alarms resulting from geologic and man-made clutter sources. Ordnance location accuracy was originally specified to be within a $0.5-\mathrm{m}$ halo (horizontally) around the emplaced munition (this corresponds to 
excavating a $0.5-\mathrm{m}$ horizontal radius hole centered at the declared location and striking any part of the UXO). Because of the highly cluttered environment, difficult site conditions (wind, terrain, heat), and uncertainties regarding the actual position of the preexisting buried UXO targets, the performance of demonstrators was also evaluated using a 1.0-m radius (Appendix C). Refer to Appendix D for analysis of mean location error scatter plots for setting the halo at $0.5 \mathrm{~m}$.

d. To provide high quality, georeferenced data for postdemonstration analysis and development of ROC curves and for broader use in the UXO technology development community.

e. To prepare technical reports to evaluate and document performance and to aid the Government in selecting effective and efficient systems for UXO detection and discrimination in difficult magnetic clutter sites such as Kaho'olawe.

\section{Physical Setup and Operation}

Because Kaho'olawe Island contains numerous live UXO, rough terrain, and some areas of thick vegetation, the sites available for testing and demonstration were very limited. As a result, this project was restricted to operating in previously cleared sites that fell within the existing QC and QA areas. Operating under these constraints, personnel from NAVEODTECHDIV and the U.S. Army Engineer Research and Development Center (ERDC), with support from onsite Navy and Contractor personnel, set up a Calibration site at the QC area and a limited set of demonstration scenarios at the QA area as shown in Chapter 3.

The UXO targets for the calibration and demonstration areas consisted of the following:

a. Inert UXO items that have been previously used at Kaho'olawe for QA/QC purposes and whose age, weathering, and condition can vary.

b. Pristine inert munitions from the Standard UXO Test Site Program which have been fabricated/procured to be as identical as feasible.

Several demonstration grids were prepared so that the only UXO targets were selected from this set of standard test targets. Representative samples of ordnance emplaced at the calibration site were made available to demonstrators for viewing and for conducting free-air and buried measurements during the demonstration phase.

The QC grids were expanded to allow installation of the new UXO items obtained from the standardized UXO Test Site stockpile. The demonstration areas were prepared by trimming and removing unwanted trees and tall grasses and by watering around any newly excavated areas to reduce evidence of site disturbance. No other physical alterations were made to the existing ranges as part of the site preparation activities conducted during 3 through 26 September 2001 . 
Descriptions of the inert UXO targets and the clutter items used for this demonstration are included in the Site Preparation Plan (NAVEODTECHDIV 2001b) which is available online as described in Appendix B. Photographs, descriptions, dimensions, and emplacement information of each target and clutter item are available as part of the information archived at this site. Briefly, the UXO targets ranged from $20-\mathrm{mm}$ projectiles buried near the surface, to $226.8 \mathrm{~kg}$ (500-lb) practice bombs buried to $2.5 \mathrm{~m}$ below the surface. Clutter items emplaced ranged from small (less than $0.5 \mathrm{~kg}$ ) to large (over $5 \mathrm{~kg}$ ) munitions fragments retrieved during UXO cleanup operations on Kaho'olawe.

Based on concerns raised during previous UXO detection and discrimination demonstrations, no unrealistic (fabricated) clutter items were used; instead actual munitions fragments from past Kaho'olawe cleanup operations were used. Also, based on previous JPG experience, all inert UXO targets that had not been previously fired or air dropped were demagnetized prior to emplacement to simulate the magnetic properties of ordnance that has been employed. In addition, precautions were taken during target emplacement to minimize surface disturbances (e.g., "bathtub" effect) that could alert the demonstrators to the presence of a buried object.

\section{Sampling Procedures}

The Demonstration Work Plan describes the procedures required for each of the demonstrations. Demonstrators were responsible for developing their specific survey plans (including lane spacing, sampling rate, number of channels recorded, calibration methods, etc.) and these procedures, together with their analysis techniques, are described in Appendix E.

Each of the demonstrators was allotted one 3-week period (Monday through Thursday) during 17 September through 30 November 2001 to complete the following activities:

a. System calibration and signature data collection activities at the calibration area during the first week.

$b$. Algorithm development and testing on Maui during the second week.

c. Field surveys of the demonstration areas and data analysis/preparation of dig lists and georeferenced maps during the third week.

\section{Analytical Procedures}

The evaluation factors, metrics, products, and procedures related to this demonstration are described in the Demonstration Workplan (NAVEODTECHDIV 2001a) and include the following information:

Factors that were measured and evaluated as part of this demonstration include:

a. Equipment setup and calibration time and man-hour requirements. 
b. Actual survey time and man-hour requirements for each of the demonstration test areas.

c. Downtime resulting from system malfunctions and maintenance requirements.

d. Reacquisition/resurvey time and man-hour requirements (if any).

e. Prioritized dig lists with associated signal strength and confidence levels.

f. Discrimination capability (ability to separate detected anomalies into UXO and non-UXO objects).

g. Identification capability (ability to classify UXO targets by class (e.g., mortar, projectile) and type (e.g., 105-mm projectile).

$h$. Predicted target location accuracy (including depth estimates).

i. Georeferenced anomaly maps.

j. Probability of Detection: $\mathrm{P}(\mathrm{det})$.

k. Probability of Discrimination: $\mathrm{P}($ disc $)$.

l. Probability of False Positives (Pfp) resulting only from the emplaced clutter items.

m. False Alarm Rates (FAR) resulting from nonemplaced items (e.g., geology and/or unknown items).

n. Total False Alarm Rates (Total FAR) resulting from emplaced clutter items and nonemplaced clutter items.

o. Operational costs.

The method for determining and documenting items $a$, $b$, and $c$ consisted of the Government onsite representatives tracking and recording the number of personnel and time spent performing each of these tasks. Adequate rest and lunch/snack breaks were provided and these times were included in the performance metrics calculations. If the demonstrator determined that he needed to resurvey any part of the test areas or any previously detected anomalies, all setup, calibration, survey, downtime, and reacquisition times and man-hour requirements were recorded individually (as in items a through c) but were compiled separately as reacquisition/resurvey time (item d).

Development and evaluation of items $e$ through $i$ were as follows:

(1) Each demonstrator was to combine the EM sensor data with the GPS and other position information to develop 2-D anomaly maps (item i) of a test area consisting of four contiguous $30-\mathrm{m}$ by $30-\mathrm{m}$ grids (Area A), a 1-hectare contiguous test area (Area B), and a test area consisting of six $30-\mathrm{m}$ by $30-\mathrm{m}$ grids (Area $\mathrm{C}$ ) as shown in Figure 12 . These anomaly maps, together with the corresponding digital geophysical sensor data were to be analyzed to identify all detected anomalies that could potentially be a buried UXO target for each of the test areas. All of these anomalies were to be tabulated into one preliminary dig sheet for each test area and were to include a suitable "signal strength" value determined and defined by each of 
the demonstrators (e.g., 1,100 ppm @ 930 Hz/quadrature phase for the GEM-3 system). Each demonstrator was required to submit a detailed description of and rationale for selecting this parameter as part of the prioritized dig list submissions described later in this document. The objective of the detection stage was to include as many anomalies in these lists as required to ensure as high a $\mathrm{P}(\mathrm{det})$ as possible for the full range of UXO targets considered.

(2) Each anomaly in each list was then to be further analyzed to develop the final dig sheets as illustrated in Table 1. The demonstrators were to refine the location $(\mathrm{x}, \mathrm{y})$ and estimate the depth $(\mathrm{z})$ of each object, attempt to separate (discriminate) UXO from clutter items, identify UXO by class and type (if possible), and rank the list in the following descending order: UXO - high confidence, UXO medium confidence, UXO - low confidence, Clutter - low confidence, Clutter - medium confidence, and Clutter - high confidence. In addition, the list was to include predicted ordnance class and type (e.g., mortar $/ 81 \mathrm{~mm}$ ) for all anomalies declared as UXO with high and medium confidence levels, and, if possible, UXO orientation (Azimuth and Inclination).

\section{Table 1}

\section{Sample Dig List}

DIG LIST: 1 Demonstrator: EMMS Test Area: A Including 20/40 mm ?: NO

\begin{tabular}{||l|l|l|l|l|l|l|l|l|l|l||}
\hline \hline Ranking & $\begin{array}{l}\text { Northing } \\
\mathrm{m}\end{array}$ & $\begin{array}{l}\text { Easting } \\
\mathrm{m}\end{array}$ & $\begin{array}{l}\text { Depth } \\
\mathrm{m}\end{array}$ & $\begin{array}{l}\text { Type } \\
\text { ordnance } \\
\text { Iclutter }\end{array}$ & Confidence & $\begin{array}{l}\text { Size/ } \\
\text { Weight }\end{array}$ & $\begin{array}{l}\text { Azimuth } \\
\text { deg }\end{array}$ & $\begin{array}{l}\text { Inclination } \\
\text { deg }\end{array}$ & Class & Type \\
\hline \hline 01 & 4309738.557 & 641594.2038 & 0.9144 & ordnance & high & large & 180 & 20 & projectile & $152 \mathrm{~mm}$ \\
\hline & & & & & & & & & & \\
\hline & & & & & & & & & & \\
\hline & & & & & & & & & & \\
\hline & & & & & & & & & & \\
\hline 050 & 4309689.964 & 641519.4151 & 0.89042 & ordnance & low & small & - & - & projectile & unknown \\
\hline & & & & & & & & & & \\
\hline \\
\hline 165
\end{tabular}

(3) Each demonstrator was then required to select a point (threshold) on each prioritized list where he would recommend that all objects at or above that point be excavated and the remainder left in place. We refer to this as the stop-dig-point. The goal of this step was to maximize the number of UXO targets above the threshold while minimizing the number of clutter items. In order to add realism to this demonstration, the following cost penalties were to be applied to this product: For every clutter item selected for "digging," a \$200 cost penalty was assigned (the average cost of excavating items at actual UXO remediation sites). If the demonstrator included any UXO items in the "no dig" portion of the list, it was assumed that the area (i.e., either the 1-hectare area, four 30-m grids, or six 30-m grids) would fail the Quality Assurance and/or regulatory 
acceptance, and a cost penalty equal to the cost of a resurvey was assigned. Missed UXO targets, that is those not included in the dig lists either as clutter or UXO, were also penalized the cost of a resurvey. Note that the same resurvey penalty was applied only once for each area whether only one or any number of UXO items were left in the ground through misdiscrimination, or by failing to detect it, or a combination of both.

(4) In order to better approximate real-world UXO cleanup operations, the Government offered to furnish ground-truth information of Area A within $24 \mathrm{hr}$ of a demonstrator's submitting prioritized dig lists for that specified portion of the test area. This procedure was intended to correspond to the additional information that is normally available to the UXO survey contractors when surveyed grids are excavated. It should be noted that groundtruth corresponding only to anomalies included in the prioritized dig lists were provided. No information on missed targets was made available until the full groundtruth was released after the completion of demonstrations. In order to provide this information as early as possible during the blind demonstrations, the following procedure was followed. At the beginning of the demonstration phase, each demonstrator was directed to survey Area A, consisting of four 30-m by $30-\mathrm{m}$ grids. The demonstrator was then to proceed to develop a prioritized dig list of this area while his survey crew continued to collect field survey data of the remaining test areas. As soon as the demonstrator submitted the prioritized dig list, the Government representative evaluated the results and provided groundtruth information on the declared target and clutter items. The demonstrator could then use that information to modify his analysis and/or survey techniques during the remainder of the blind demonstration. The goal was for each demonstrator to have this information prior to starting the analysis of the 1-hectare site. It should be noted that the majority of demonstrators did not submit the dig lists for Area A until the end of the demonstrations and, therefore, did not receive groundtruth information to aid in the analysis of Areas B and C.

(5) Items $j$ through $n$ were calculated from the prioritized dig lists as follows: Maximum achievable $\mathrm{P}(\mathrm{det})$ for each area was calculated as the number of items in the entire list that correspond to emplaced UXO targets (even though they may have been misdiscriminated as clutter) divided by the actual number of UXO targets emplaced in that site. Similarly, maximum achievable P(disc) was calculated as the number of anomalies in the dig list that were correctly classified as UXO divided by the total number of emplaced UXO targets. In addition, the single point probability of discrimination ( $\mathrm{P}($ disc $)$ ) was determined by calculating the number of actual UXO targets that are correctly classified as UXO and are included in the list at or above the specified dig point. The operating (single point) FAR was calculated as the number of items per surveyed area that were included above the dig threshold and which did not correspond to emplaced target or emplaced clutter locations. FAR is therefore a 
measure of the false positives because of natural geologic/ environmental factors and any preexisting metal objects. In addition, Pfp was calculated as the ratio of the number of clutter items declared as UXO to the number of clutter items emplaced. Total False Alarm Rates (item $n$ ) were computed by combining both false alarm sources that make up items $l$ and $m$. ROC-like curves were developed by the Government by varying the dig threshold until the maximum $\mathrm{P}($ det $)$ and $\mathrm{P}$ (disc) were reached, and plotting $\mathrm{P}($ det $), \mathrm{P}($ disc $)$ as the ordinate and Pfp and FAR as the abscissa at each increment. ROC-like curves of $\mathrm{P}(\mathrm{det})$ and $\mathrm{P}($ disc $)$ vs. Total FAR were also developed by using the specified "signal strength" parameter as the thresholding variable. Performance comparisons between systems include using the ROC-like curves to determine Pfp and FAR at the P(det) required for Kaho'olawe Tier II clearance (85 percent).

(6) After each demonstrator had submitted the dig sheets described above, a total of three dig lists, he/she was given the opportunity to reanalyze the data from the 1-hectare site and the six $30-\mathrm{m}$ by $30-\mathrm{m}$ grids to develop two additional prioritized dig sheets that take into account only targets larger than 40-mm projectiles. All dig sheets were to be submitted to the Government representative within 3 days after completing the field demonstration and prior to departing Hawaii. After all field demonstrations were completed, the ESTCP Program Office provided each of the demonstrators with the complete groundtruth for all of the test areas at both JPG and Kaho'olawe. Each demonstrator was then required to reanalyze his/her results, identify problems and potential improvements, and submit a self-evaluation draft report to the ESTCP Program Office. This draft report will be due no later than 2 months after release of the groundtruth. The ESTCP Program Office will review the draft report and return it to the demonstrator with comments. A final report will be due no later than 30 days after receiving comments from the ESTCP Program Office.

(7) Item $o$, Operational costs were estimated using the cost factors and procedures described in Chapter 6. 


\section{Performance Assessment}

\section{Introduction}

In accordance with the Demonstration Plan, each of the demonstrators was responsible for determining the best method of employing his system in order to: (a) ensure full coverage of each demonstration area, (b) collect high-quality sensor data to support detection and discrimination requirements, (c) achieve high production rates, and (d) minimize man-hour requirements and costs. All demonstrators were able to complete the field surveys within the allotted time periods.

There was a wide range in the demonstrators' survey data quality, data density, quality of analysis, and compliance with the data submission requirements specified in the Demonstration Plan (NAVEODTECHDIV 2001a). For example, a number of demonstrators failed to include required dig list information such as recommended stop dig point, appropriate confidence levels, and signal strength levels, and most demonstrators failed to reanalyze their data and prepare dig lists that excluded the small $20 \mathrm{~mm}$ and $40 \mathrm{~mm}$ targets. This lack of adherence to the requirements of the Demonstration Plan has made the interpretation of results and adequate across-demonstrator performance comparisons very difficult. This Chapter presents a summary of the data submitted by the demonstrators and the Government's assessment of their performance.

It should be noted that because of the very high numbers of false alarms submitted by all demonstrators, the Government was not able to fully investigate the sources of all of them. Nevertheless, during April 2002, NAVEODTECHDIV personnel conducted extensive surveys and excavation activities in the calibration and demonstration areas in order to verify the emplaced target locations and to attempt to identify the sources of a large percentage of the false alarms. Information from these postdemonstration activities was incorporated into the groundtruth data used to evaluate the demonstrators' performance.

During the verification of the emplaced target locations, the locations of the following targets were updated: 1A 87, 1A 204, 1A 225, 1E 95, 1E 103, 2E 96, 3D 691, 4D 116, 4D 169, and 4D 89. All of these targets were previously emplaced targets on the site.

While identifying sources of false alarms, 16 items were added to the groundtruth. Eleven of the items were considered to be no-grade items and were removed from evaluation. Their labels are $1 \mathrm{C} \mathrm{C} 8,1 \mathrm{C} \mathrm{C} 48,1 \mathrm{C} \mathrm{C} 49,1 \mathrm{D}$ A7, 1D 
$\mathrm{A} 11,2 \mathrm{~A} \mathrm{C} 15,2 \mathrm{~B} \mathrm{C} 34,2 \mathrm{~B} \mathrm{C} 42,3 \mathrm{~B} \mathrm{~B} 16,3 \mathrm{E} \mathrm{B} 6$, and 3E B7. The other five items were graded as ordnance. Their labels are 3B B14, 3B B17, 4B B3, 5B B32, and $5 \mathrm{C}$ B36.

\section{Anomaly Maps}

Figures 16 through 31 show the geo-referenced anomaly maps produced by each of the systems used during these demonstrations. Parsons did not submit an anomaly map of Area A for EM-61 Digital; however, they did submit a dig sheet for this area.

\section{Detection Results}

Table 2 summarizes the number of UXO targets detected within $0.5-\mathrm{m}$ circular error by each demonstrator. The maximum achievable probability of detection, $\mathrm{P}(\mathrm{det})$, is also given in the table. The $\mathrm{P}(\mathrm{det})$ is calculated as the number of declared items that correspond to emplaced UXO targets (even though they may have been misclassified as clutter) divided by the actual number of UXO targets emplaced in the demonstration site.

\begin{tabular}{|c|c|c|c|c|c|}
\hline \multicolumn{6}{|c|}{$\begin{array}{l}\text { Table } 2 \\
\text { P(det) by Area within } 0.5 \mathrm{~m}\end{array}$} \\
\hline & & \multicolumn{4}{|c|}{ Within $0.5 \mathrm{~m}$} \\
\hline & & Area A & Area B & Area C & Total \\
\hline & $\begin{array}{l}\text { Number of Actual } \\
\text { Targets }\end{array}$ & 24 & 81 & 34 & 139 \\
\hline \multirow[t]{2}{*}{ NAEVA } & Targets Detected & 13 & 33 & 10 & 56 \\
\hline & $P($ det $)$ & 0.542 & 0.407 & 0.294 & 0.403 \\
\hline \multirow[t]{2}{*}{ GTL } & Targets Detected & 10 & 30 & 3 & 43 \\
\hline & $P($ det $)$ & 0.417 & 0.370 & 0.088 & 0.309 \\
\hline \multirow[t]{2}{*}{ Geophex } & Targets Detected & 16 & 33 & 10 & 59 \\
\hline & $\mathrm{P}(\mathrm{det})$ & 0.667 & 0.407 & 0.294 & 0.424 \\
\hline \multirow[t]{2}{*}{ NRL } & Targets Detected & 8 & 17 & 6 & 31 \\
\hline & $\mathrm{P}($ det $)$ & 0.333 & 0.210 & 0.176 & 0.223 \\
\hline \multirow[t]{2}{*}{ NRL without $20 / 40 \mathrm{~mm}$} & Targets Detected & 9 & 20 & 8 & 37 \\
\hline & $P($ det $)$ & 0.375 & 0.247 & 0.235 & 0.266 \\
\hline \multirow[t]{2}{*}{$\begin{array}{l}\text { Parsons EM-61 } \\
\text { EM and Flag }\end{array}$} & Targets Detected & 12 & 33 & 15 & 60 \\
\hline & $\mathrm{P}($ det $)$ & 0.500 & 0.407 & 0.441 & 0.432 \\
\hline \multirow[t]{2}{*}{ Parsons EM-61 Digital } & Targets Detected & 12 & 25 & 15 & 52 \\
\hline & $\mathrm{P}($ det $)$ & 0.500 & 0.309 & 0.441 & 0.374 \\
\hline \multirow[t]{2}{*}{ Parsons TM-5 EMU } & Targets Detected & 6 & 13 & 1 & 20 \\
\hline & $P($ det $)$ & 0.250 & 0.160 & 0.029 & 0.144 \\
\hline
\end{tabular}




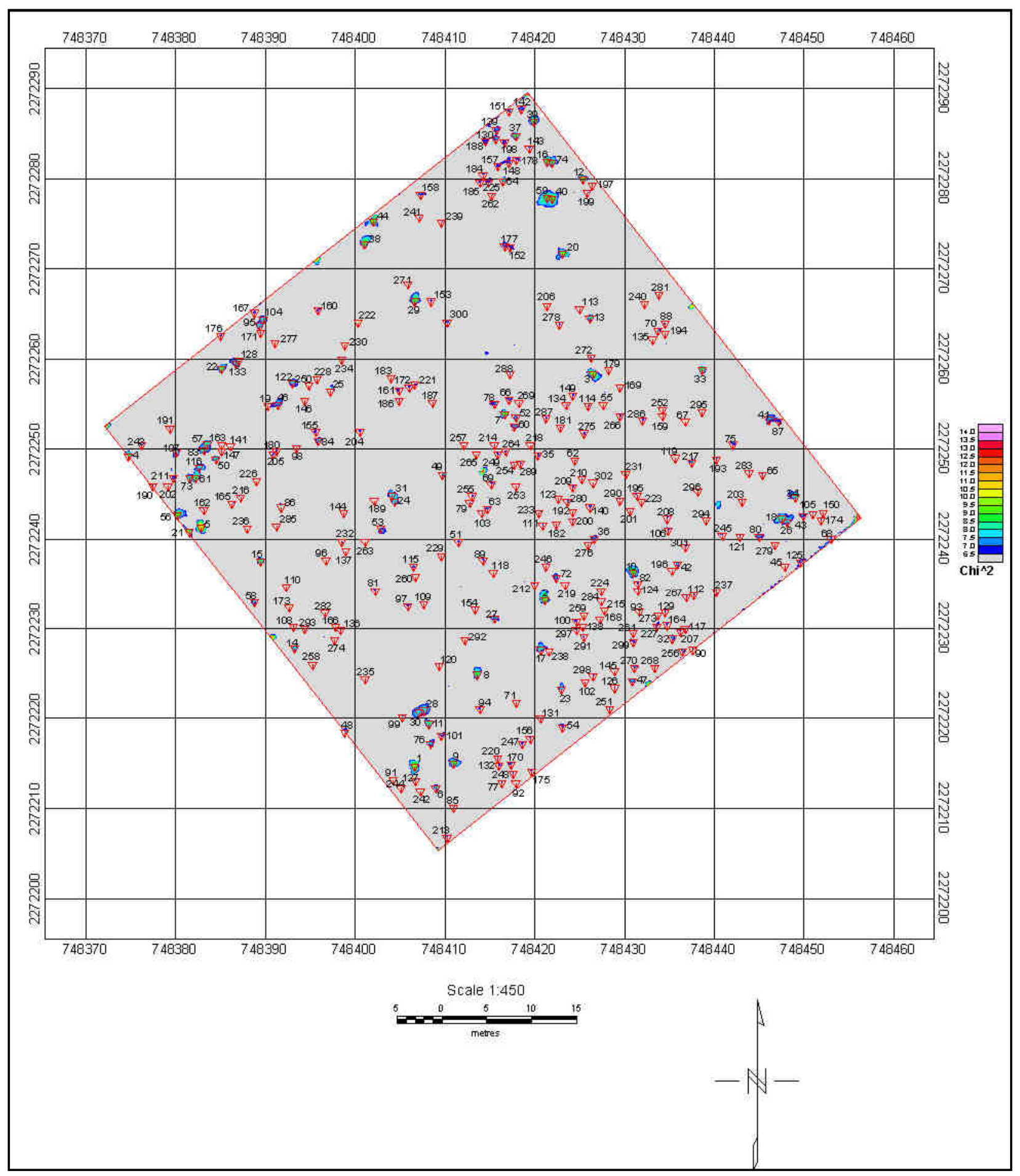

Figure 16. NAEVA EM-63 survey of Area A 


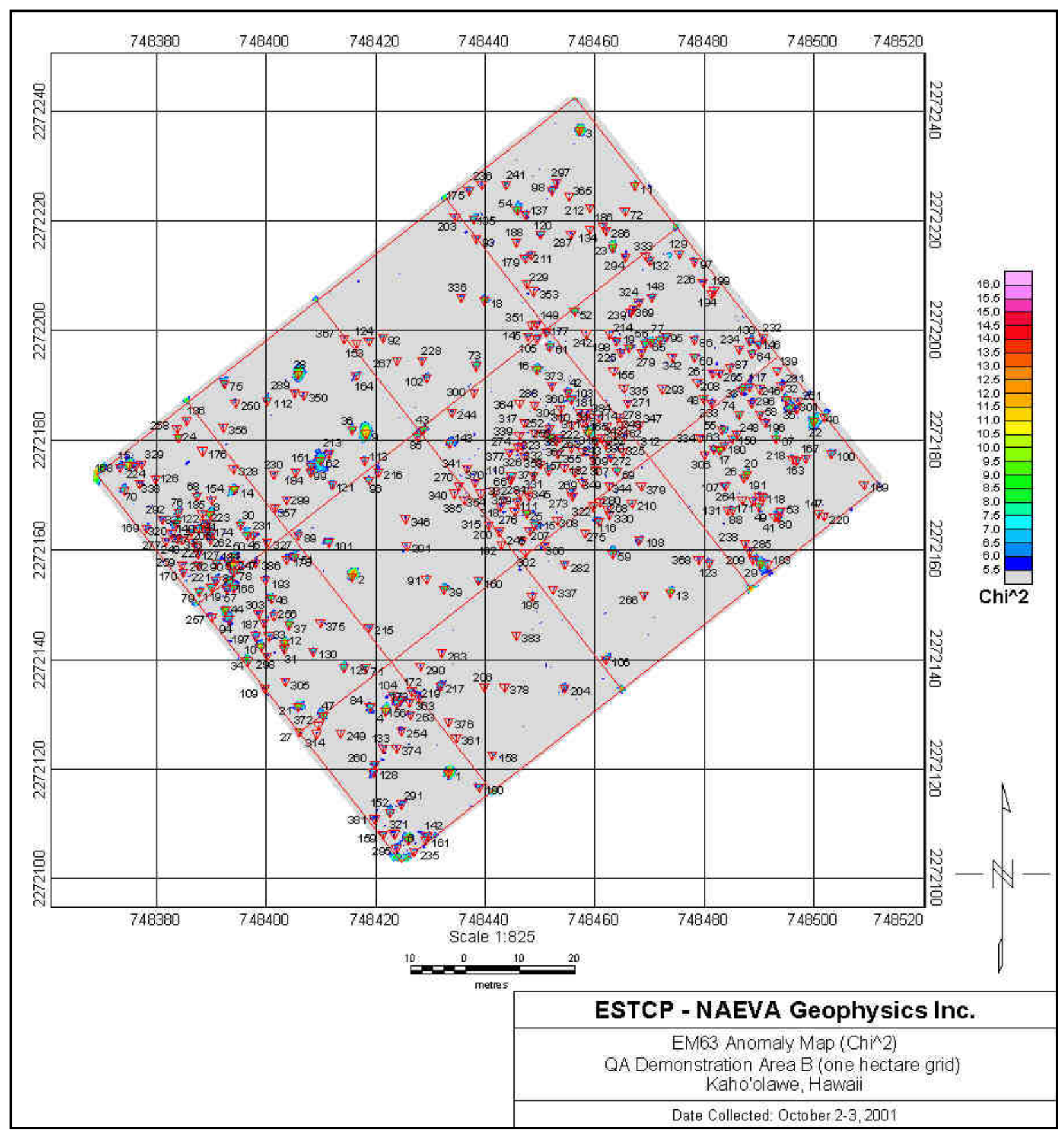

Figure 17. NAEVA EM-63 survey of Area B 


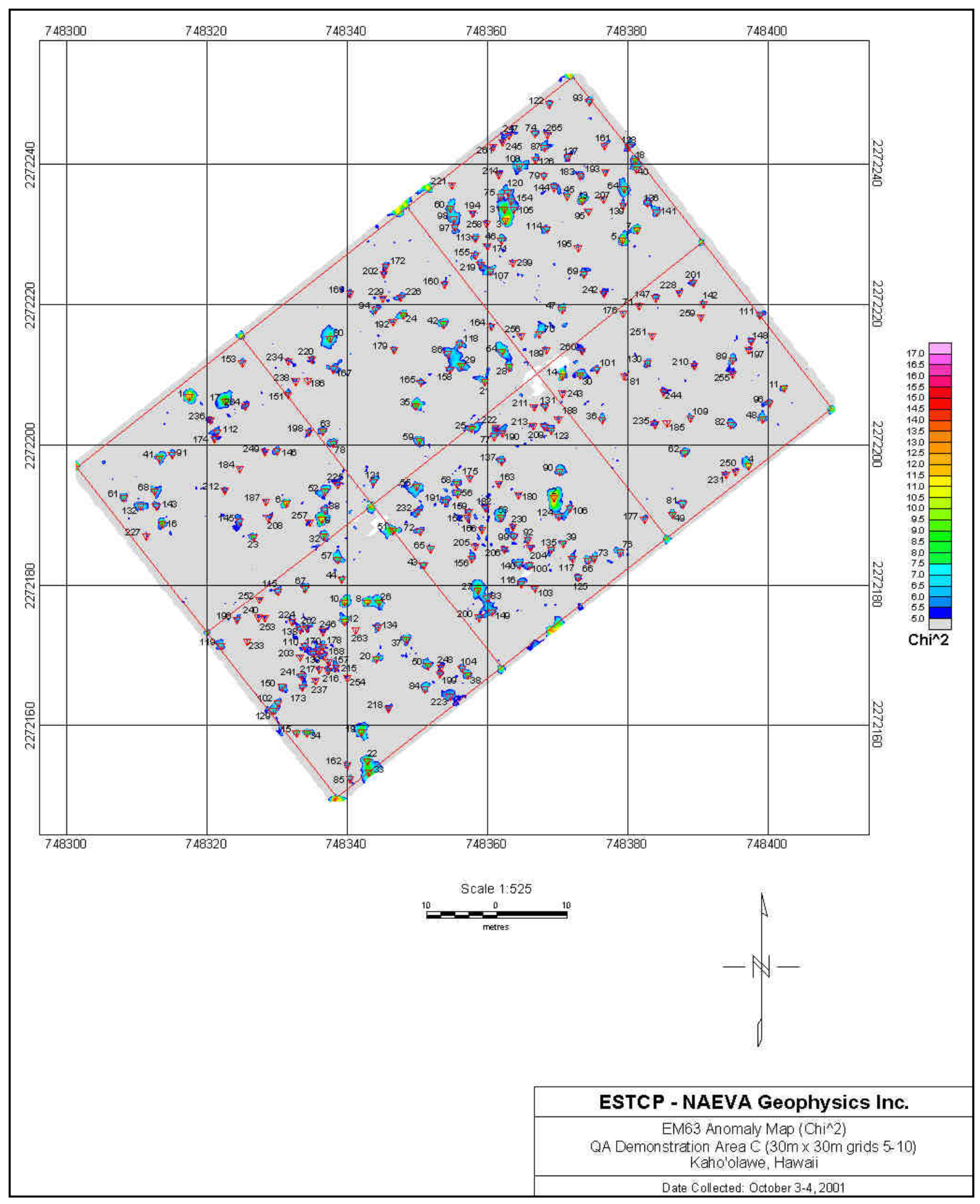

Figure 18. NAEVA EM-63 survey of Area C 


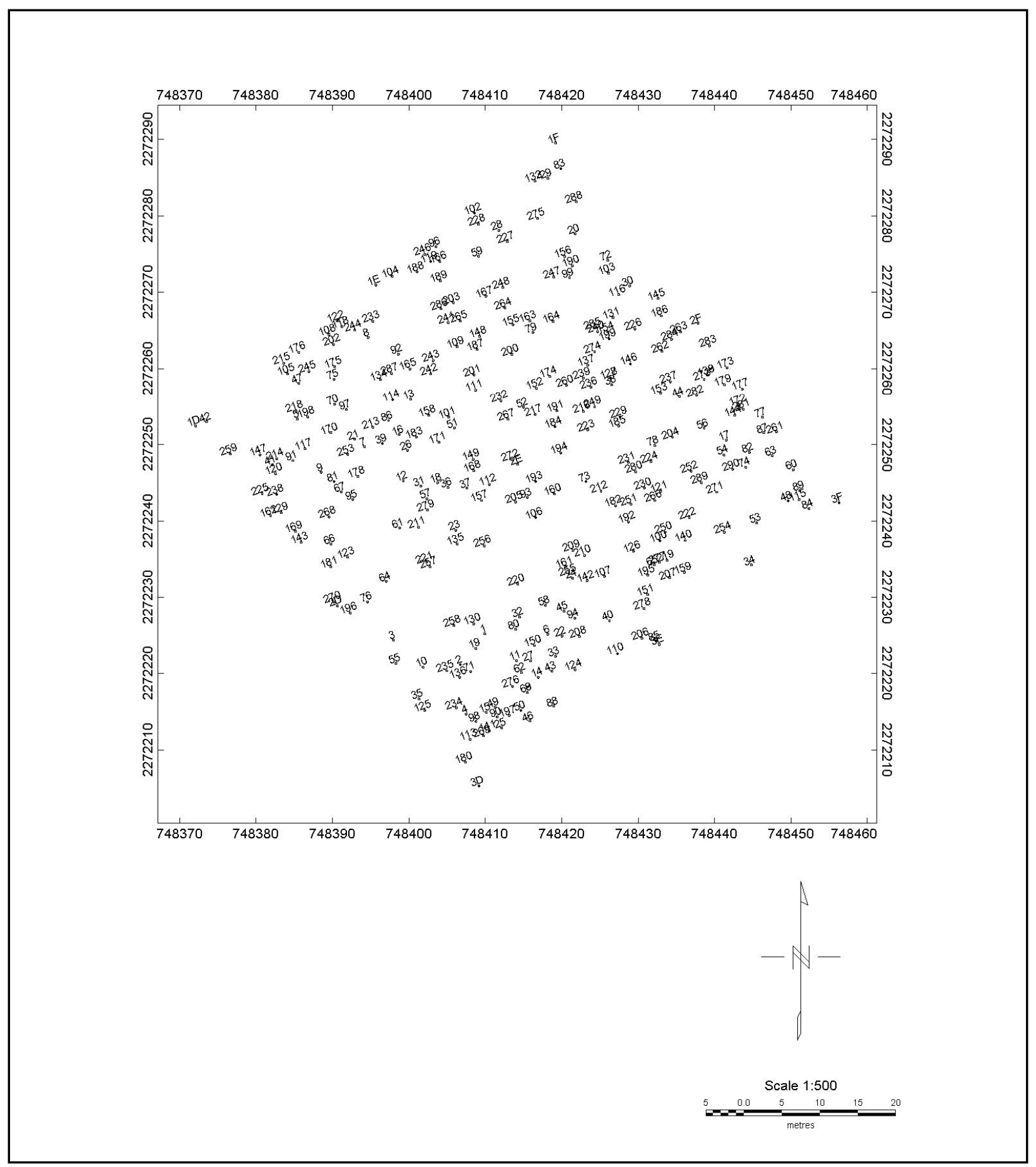

Figure 19. GTL TM-5 EMU survey of Area A 


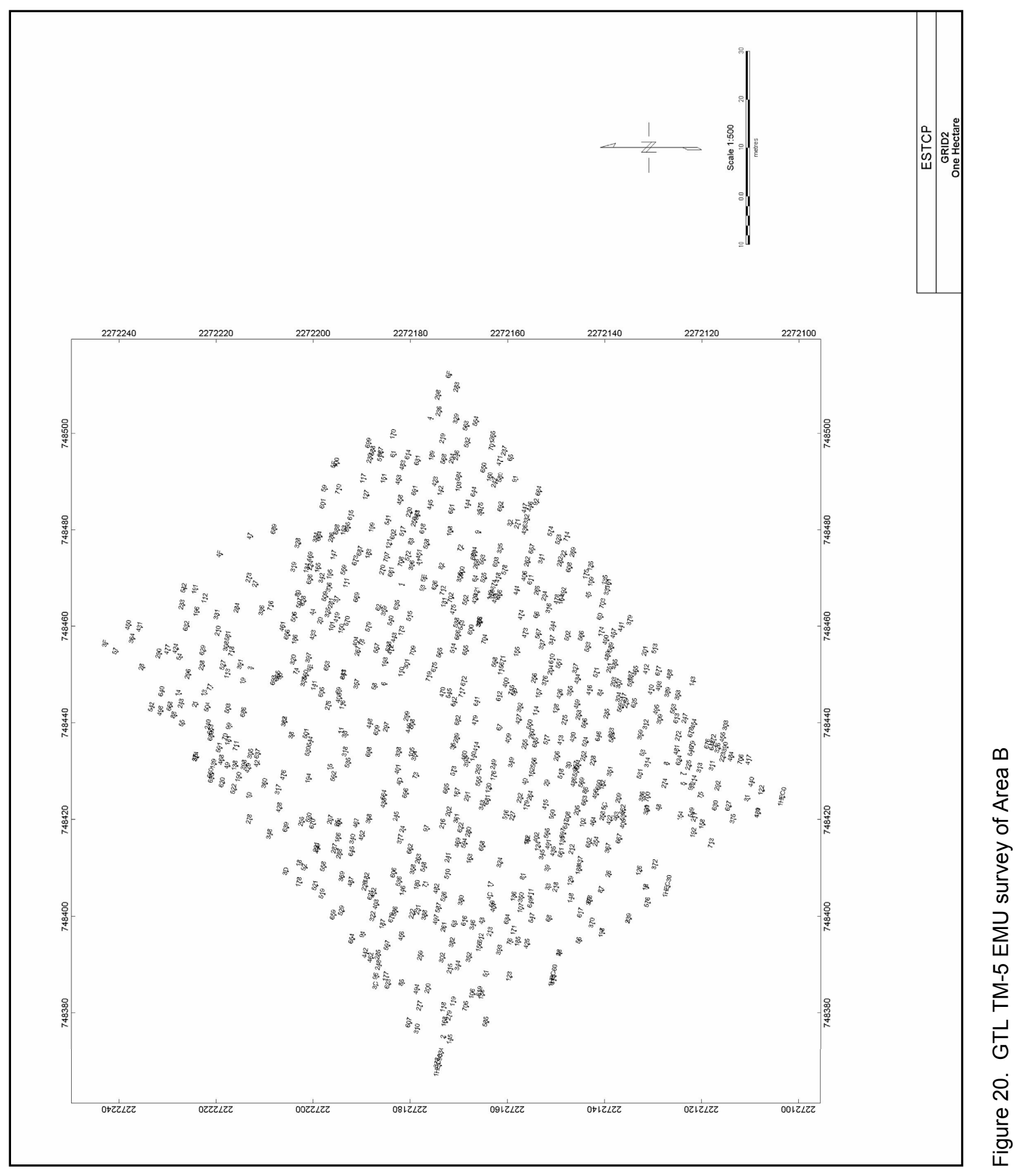




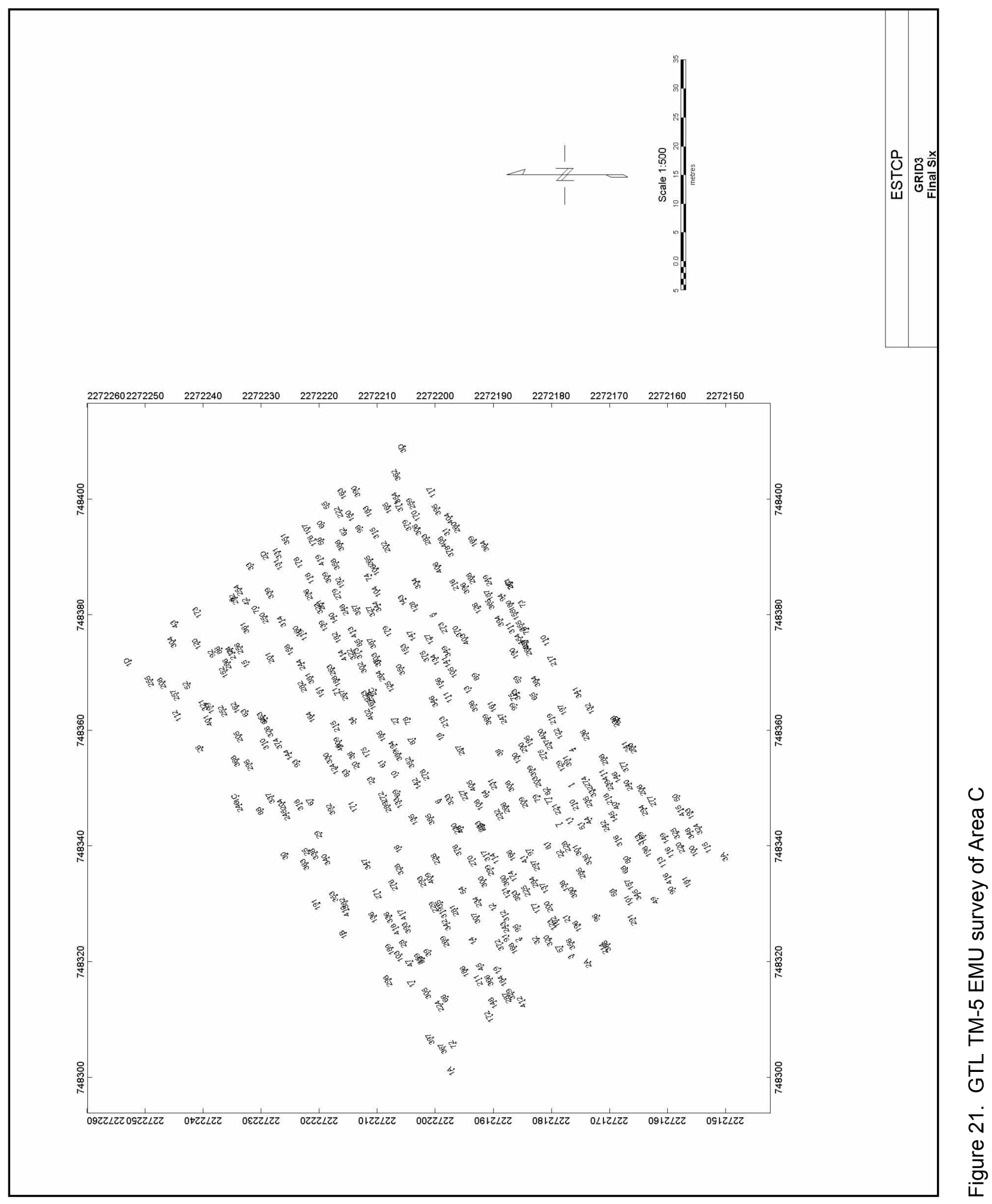




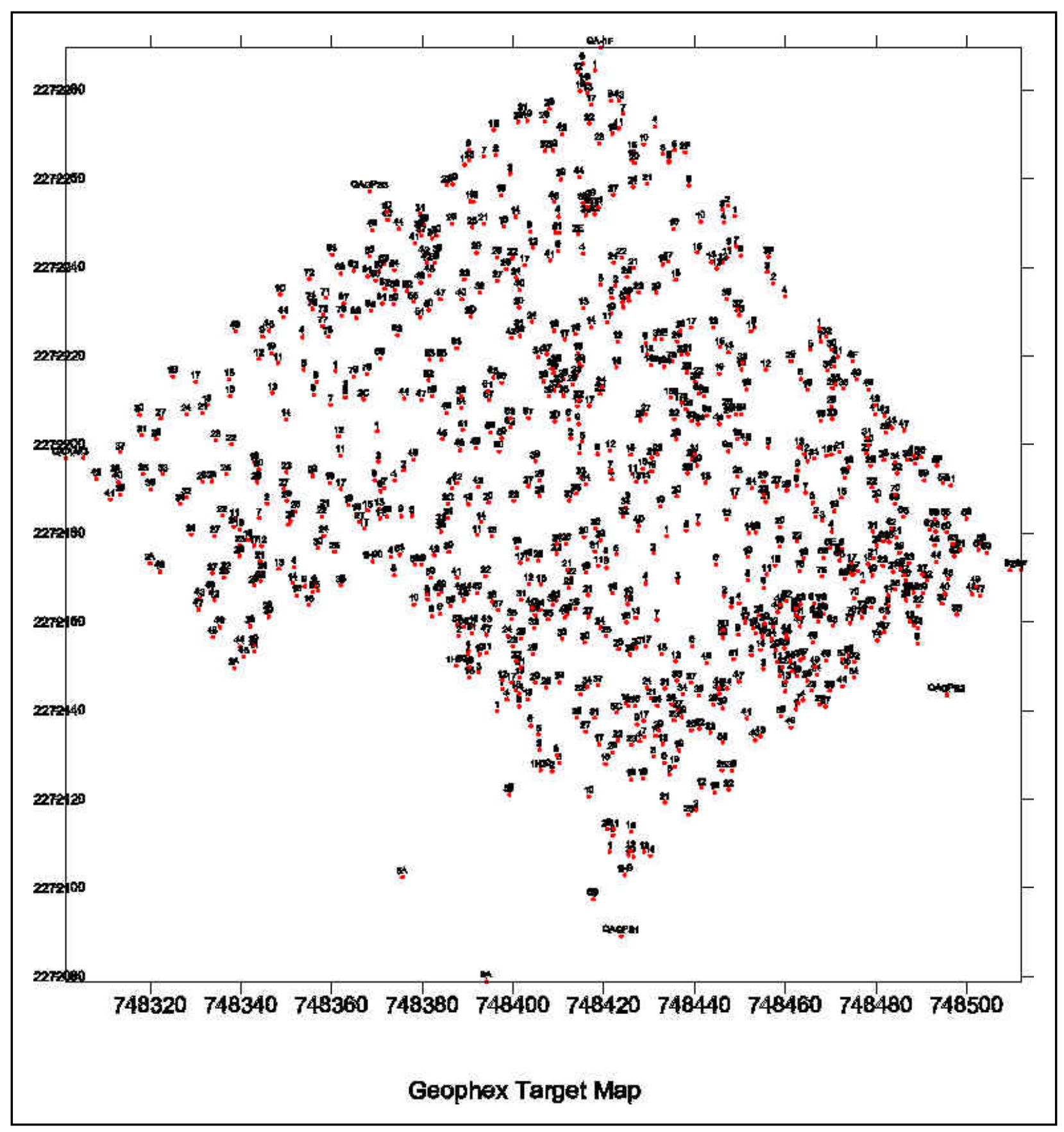

Figure 22. Geophex, Ltd. GEM-3 survey of Areas A, B, and C 


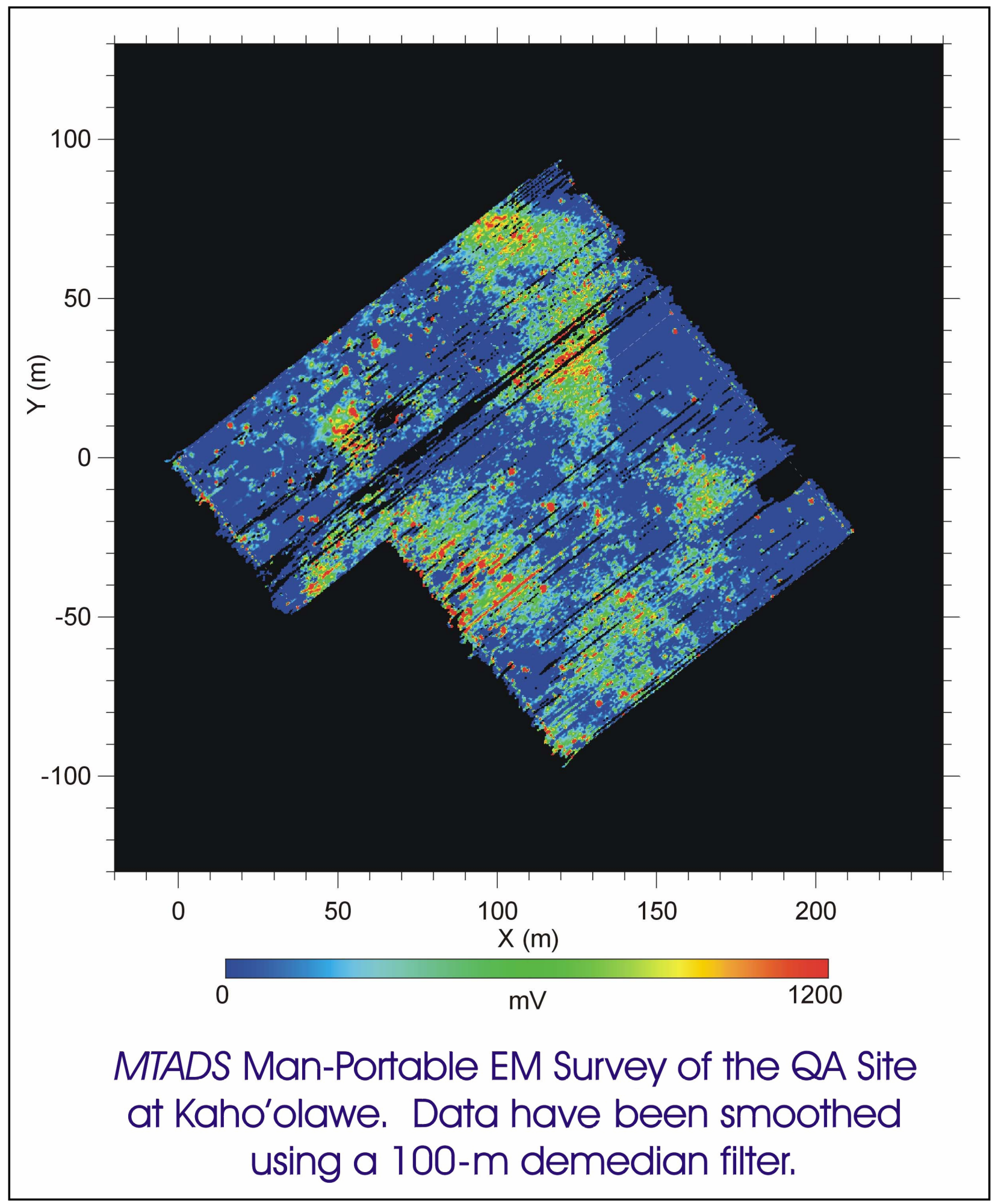

Figure 23. NRL EMMS survey of Areas A, B, and C 


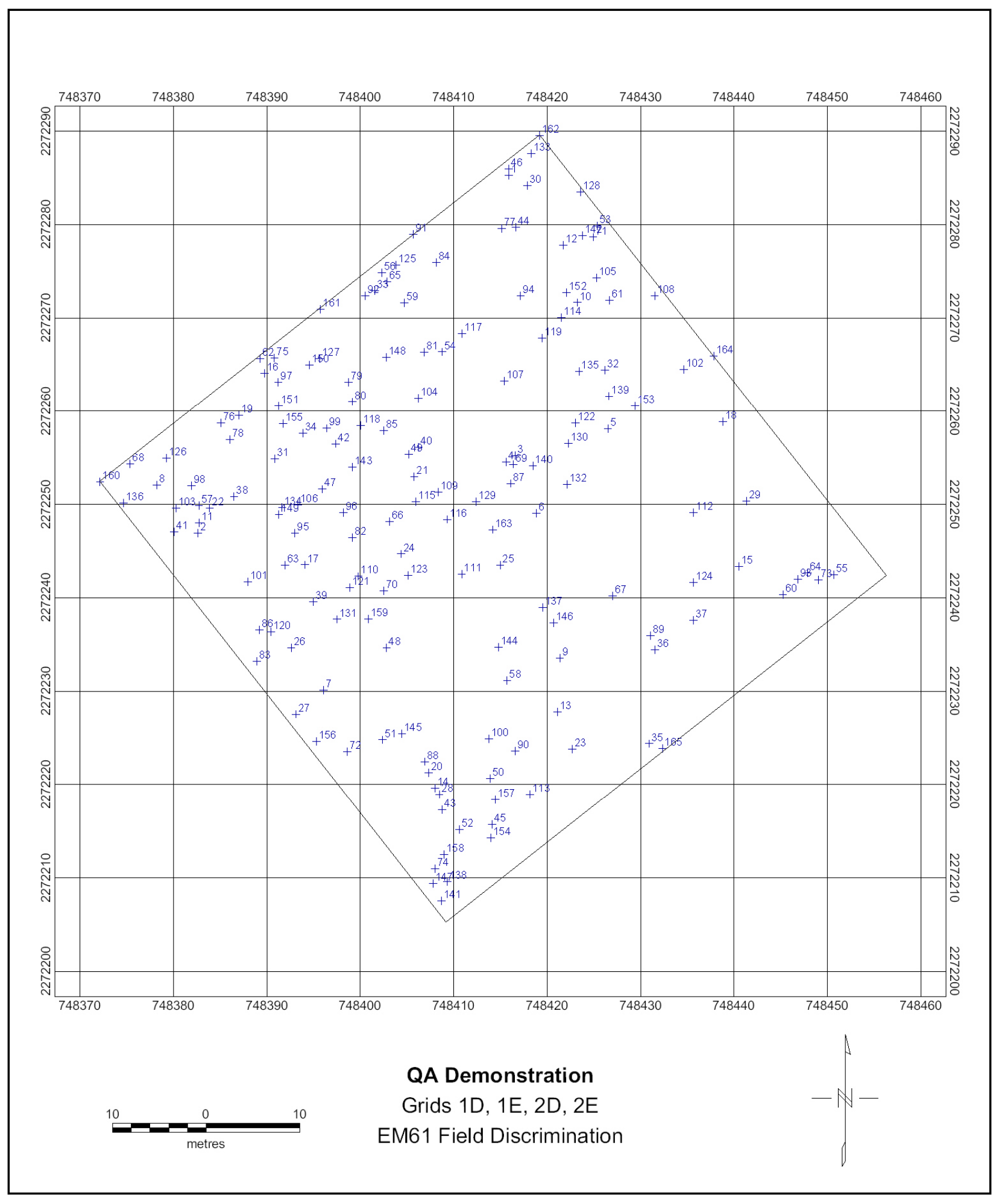

Figure 24. Parsons EM and Flag survey of Area A 


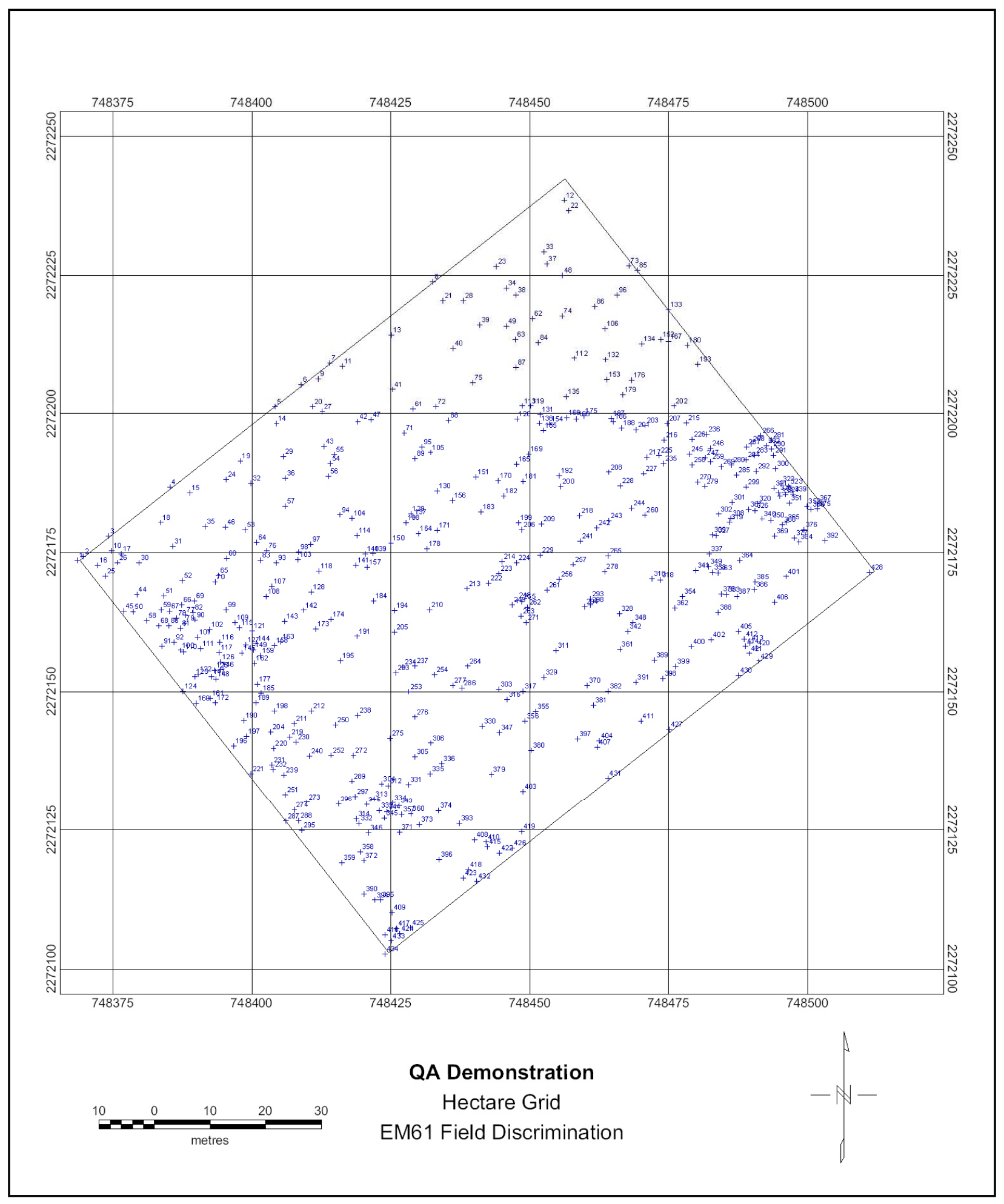

Figure 25. Parsons EM and Flag survey of Area B 


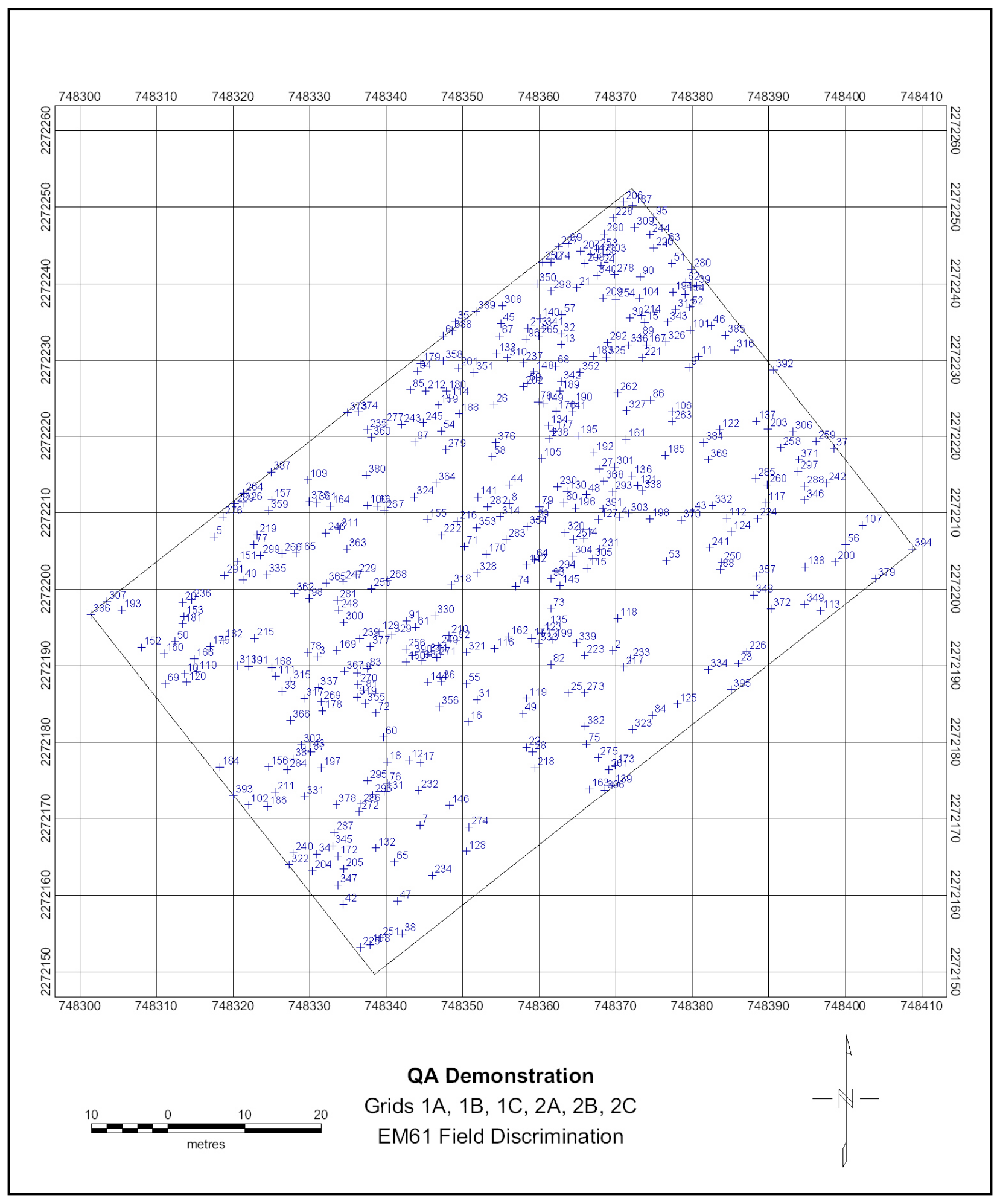

Figure 26. Parsons EM and Flag survey of Area C 


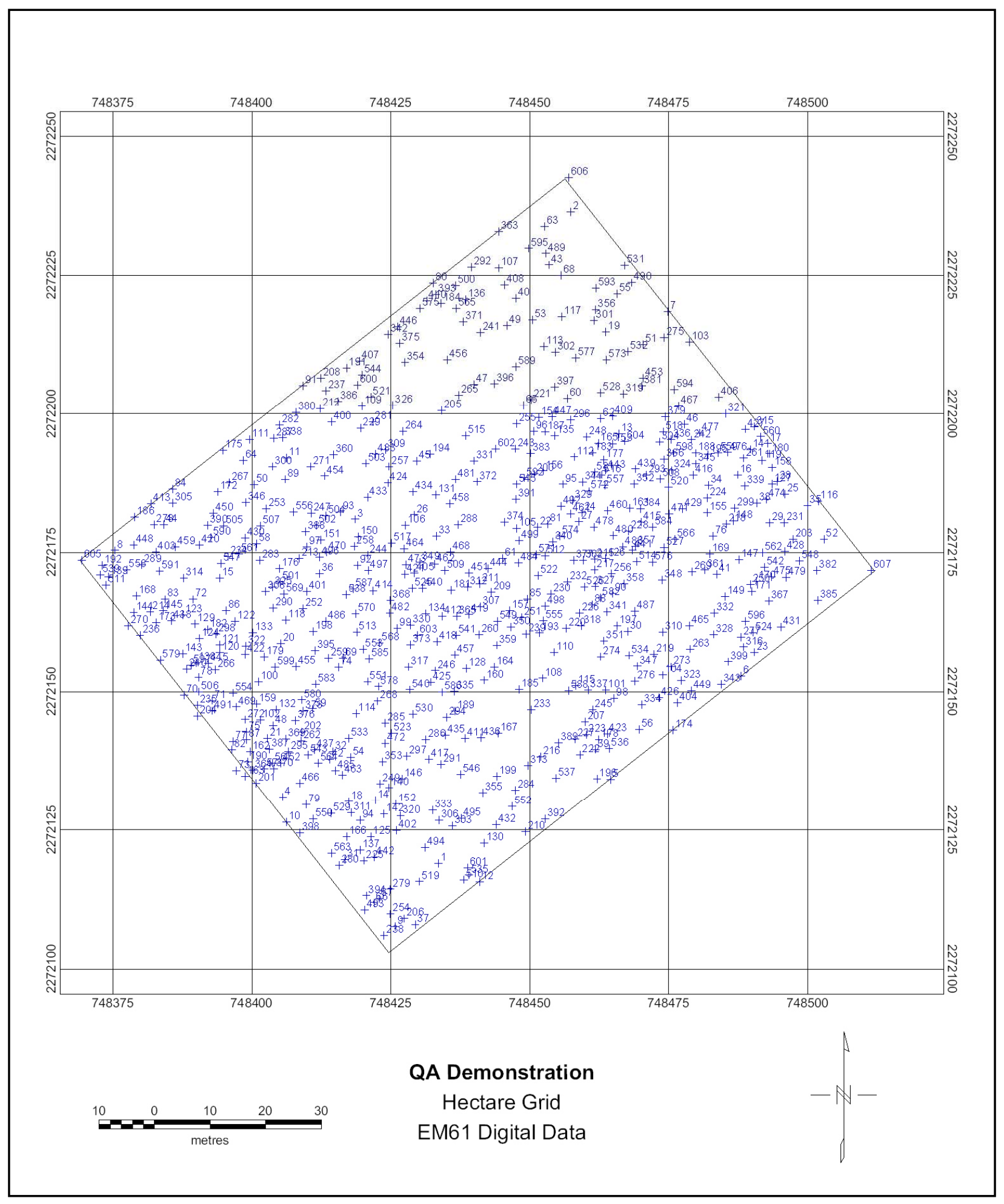

Figure 27. Parsons EM-61 Digital survey of Area B 


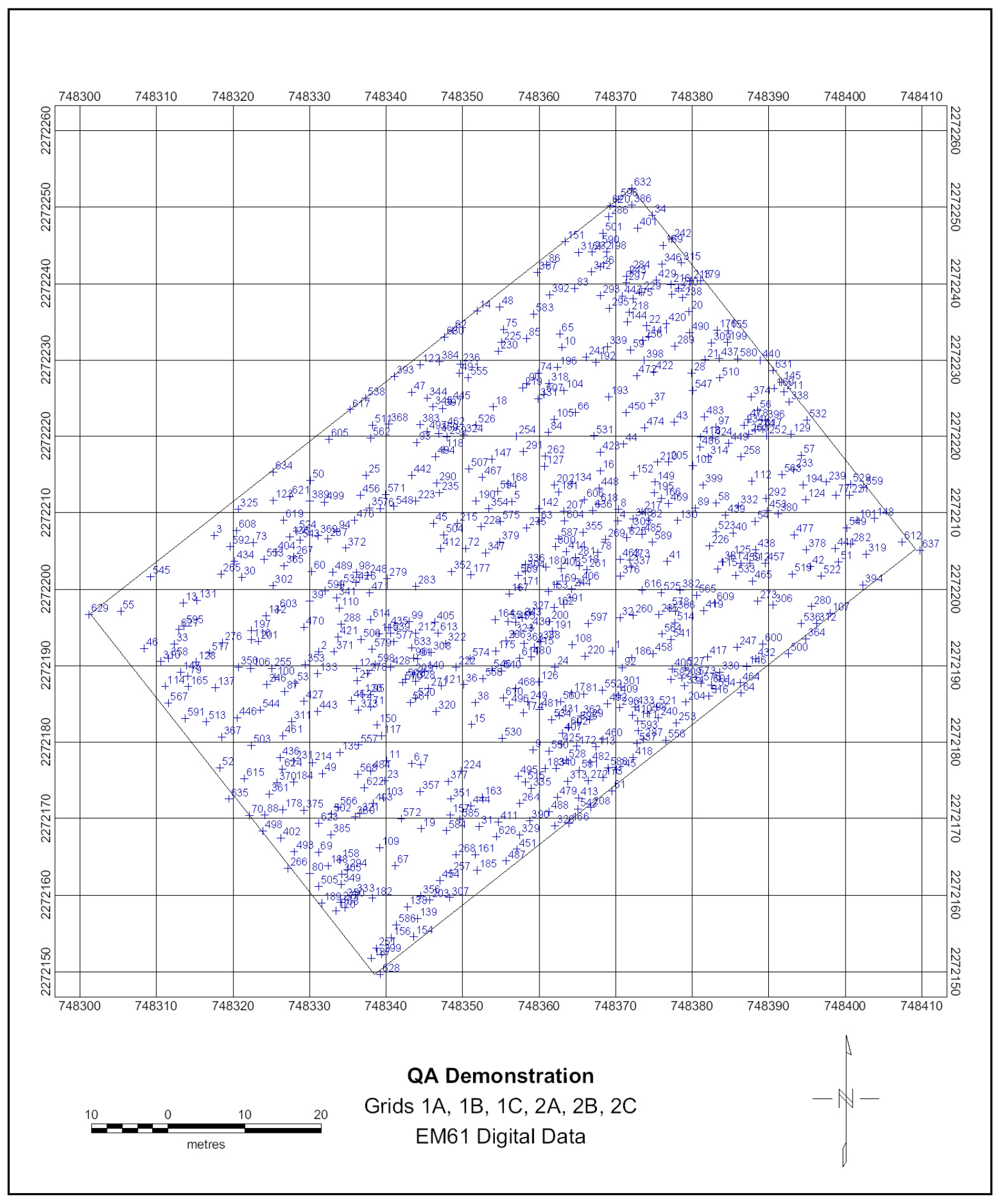

Figure 28. Parsons EM-61 Digital survey of Area C 


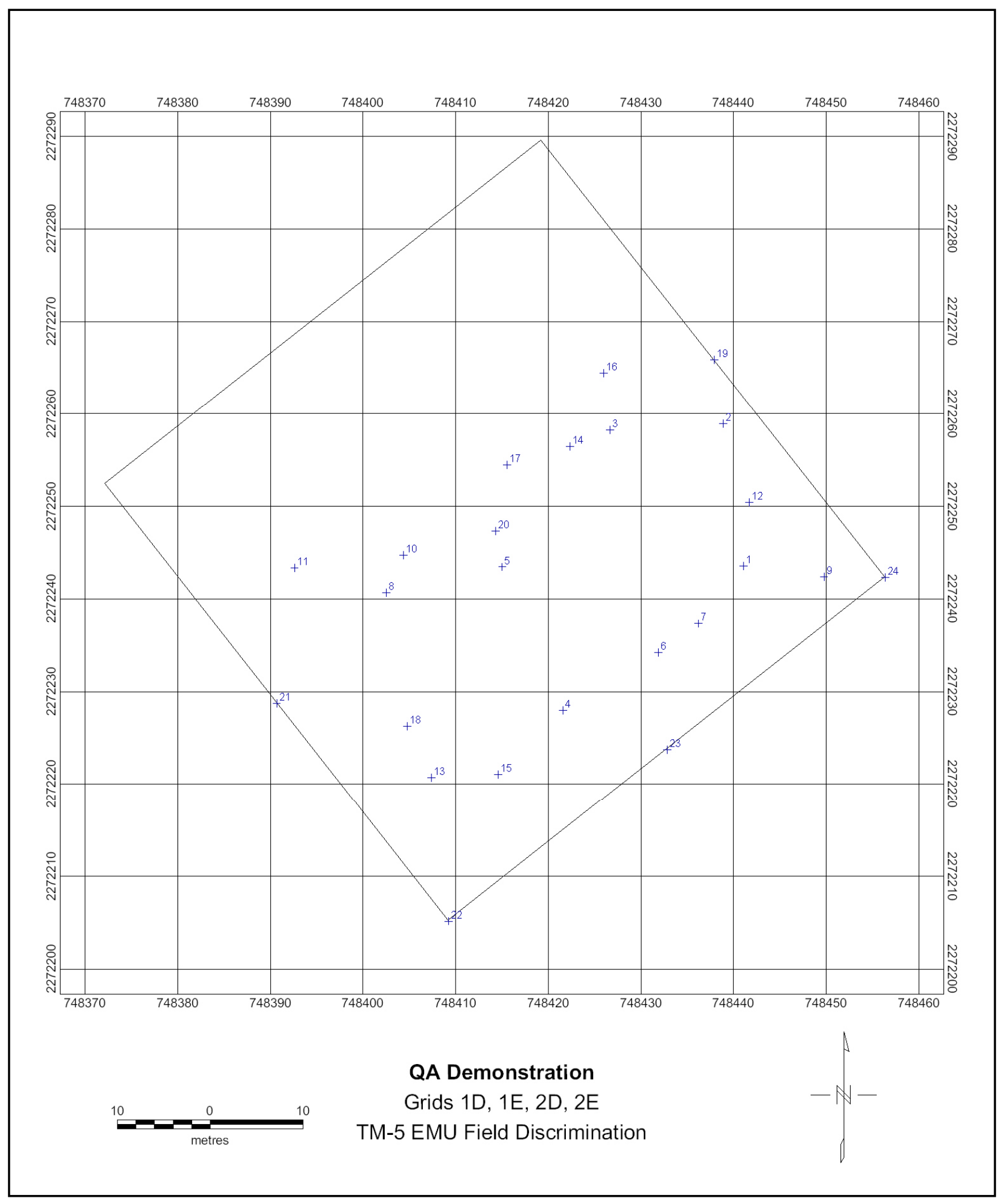

Figure 29. Parsons TM-5 EMU survey of Area A 


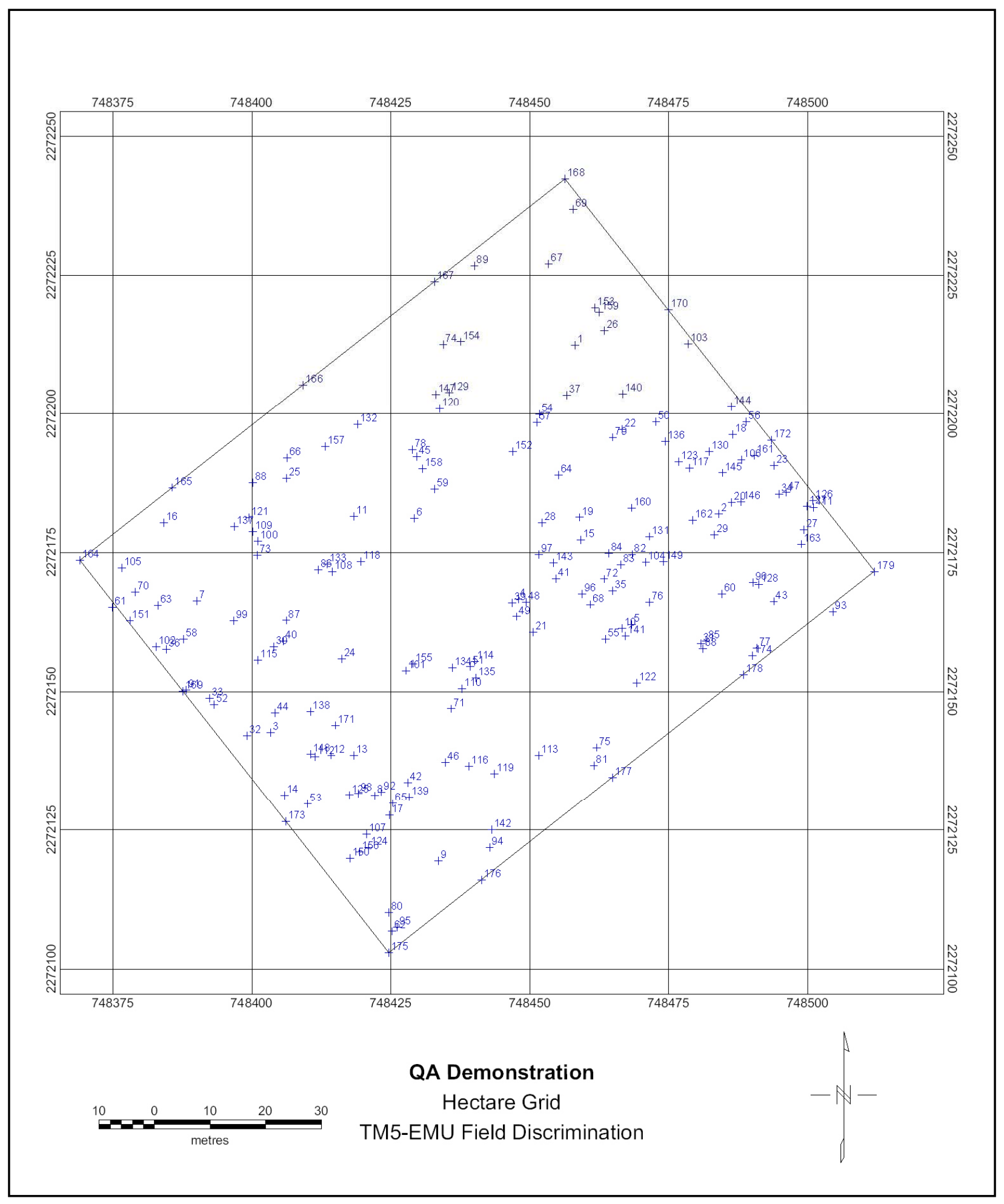

Figure 30. Parsons TM-5 EMU survey of Area B 


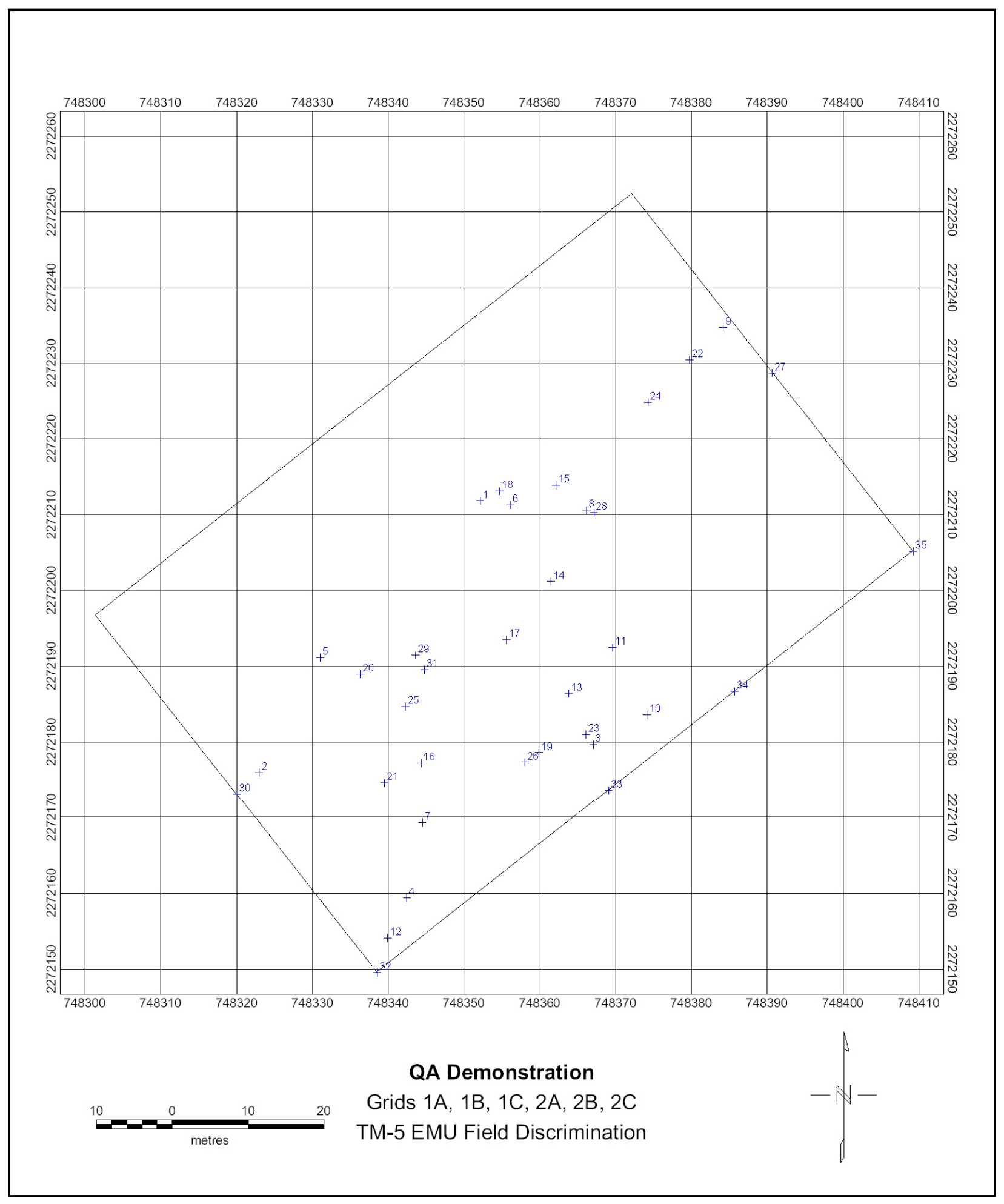

Figure 31. Parsons TM-5 EMU survey of Area C 
Table 3 summarizes the detection results achieved when the small UXO targets (20-mm and 40-mm projectiles) were excluded from the evaluation. The detection results within a 1.0-m circular error can be found in Appendix C. These results indicate that no demonstrator was able to achieve the Kaho'olawe Tier II clearance requirements of $0.85 \mathrm{P}(\mathrm{det})$ with a $0.5-\mathrm{m}$ location accuracy at any of the three demonstration areas. Only when the requirements were relaxed by expanding the allowable position error to $1.0 \mathrm{~m}$ and also deleting the smaller UXO targets did any of the demonstrators meet the $\mathrm{P}(\mathrm{det})$ requirements (see Appendix C, Table C1-C2). Even then, acceptable P(det) levels were only obtained in Area A which had considerably lower levels of geologic noise and metallic clutter than the other two Areas.

\begin{tabular}{|c|c|c|c|c|c|}
\hline \multicolumn{6}{|c|}{$\begin{array}{l}\text { Table } 3 \\
\mathrm{P} \text { (det) by Area within } 0.5 \mathrm{~m} \text { and without } 20 / 40 \mathrm{~mm}\end{array}$} \\
\hline & & \multicolumn{3}{|c|}{ Within $0.5 \mathrm{~m}$} & \multirow[b]{2}{*}{ Total } \\
\hline & & Area A & Area B & Area C & \\
\hline & $\begin{array}{l}\text { Number of Actual } \\
\text { Targets without } \\
20 / 40 \mathrm{~mm}\end{array}$ & 19 & 55 & 28 & 102 \\
\hline \multirow[t]{2}{*}{ NAEVA } & Targets Detected & 11 & 26 & 9 & 46 \\
\hline & $\mathrm{P}(\mathrm{det})$ & 0.579 & 0.473 & 0.321 & 0.451 \\
\hline \multirow[t]{2}{*}{ GTL } & Targets Detected & 9 & 24 & 3 & 36 \\
\hline & $P($ det $)$ & 0.474 & 0.436 & 0.107 & 0.353 \\
\hline \multirow[t]{2}{*}{ Geophex } & Targets Detected & 13 & 28 & 9 & 50 \\
\hline & $\mathrm{P}(\mathrm{det})$ & 0.684 & 0.509 & 0.321 & 0.490 \\
\hline \multirow[t]{2}{*}{ NRL } & Targets Detected & 7 & 17 & 6 & 30 \\
\hline & $P($ det $)$ & 0.368 & 0.309 & 0.214 & 0.294 \\
\hline \multirow[t]{2}{*}{ NRL without $20 / 40 \mathrm{~mm}$} & Targets Detected & 8 & 20 & 8 & 36 \\
\hline & $P($ det $)$ & 0.421 & 0.364 & 0.286 & 0.353 \\
\hline \multirow[t]{2}{*}{$\begin{array}{l}\text { Parsons EM-61 } \\
\text { EM and Flag }\end{array}$} & Targets Detected & 11 & 26 & 15 & 52 \\
\hline & $P($ det $)$ & 0.579 & 0.473 & 0.536 & 0.510 \\
\hline \multirow[t]{2}{*}{ Parsons EM-61 Digital } & Targets Detected & 10 & 24 & 15 & 49 \\
\hline & $P($ det $)$ & 0.526 & 0.436 & 0.536 & 0.480 \\
\hline \multirow[t]{2}{*}{ Parsons TM-5 EMU } & Targets Detected & 6 & 12 & 1 & 19 \\
\hline & $\mathrm{P}($ det $)$ & 0.316 & 0.218 & 0.036 & 0.186 \\
\hline
\end{tabular}

Table 4 shows the number of UXO targets detected within $0.5 \mathrm{~m}$ by each demonstrator in each $30-\times 30-\mathrm{m}$ grid. Shaded entries indicate the highest number of detections for that grid. The last column of the table indicates the number of UXO targets not detected by any of the demonstrators. Tables 5 through 12 show detections by ordnance type for each demonstrator. Sixteen different ordnance types are listed in each table. No UXO targets of the other two types listed in the demonstration plan (BDU 3 practice bomb and 3-in. projectile) were emplaced for this demonstration. 


\begin{tabular}{|c|c|c|c|c|c|c|c|c|c|}
\hline \multicolumn{10}{|c|}{$\begin{array}{l}\text { Table } 4 \\
\text { Grid-by-Grid Detections within } 0.5 \mathrm{~m}\end{array}$} \\
\hline Grids & $\begin{array}{l}\text { Total Items } \\
\text { Buried } \\
\end{array}$ & NAEVA & GTL & Geophex & NRL & $\begin{array}{ll}\text { Parsons } \\
\text { EM-61 } \\
\text { EM and } \\
\text { Flag } \\
\end{array}$ & \begin{tabular}{|l} 
Parsons \\
EM-61 \\
Digital \\
\end{tabular} & \begin{tabular}{|l} 
Parsons \\
TM-5 EMU
\end{tabular} & $\begin{array}{l}\text { Items Not } \\
\text { Found }\end{array}$ \\
\hline $1 \mathrm{~A}$ & 6 & 4 & 0 & 4 & 3 & 4 & 5 & 0 & 0 \\
\hline $1 \mathrm{~B}$ & 5 & 1 & 1 & 2 & 1 & 3 & 2 & 0 & 2 \\
\hline $1 \mathrm{C}$ & 5 & 0 & 0 & 0 & 1 & 2 & 3 & 0 & 1 \\
\hline $1 \mathrm{D}$ & 4 & 3 & 2 & 4 & 0 & 2 & 1 & 0 & 0 \\
\hline $1 \mathrm{E}$ & 7 & 4 & 2 & 5 & 5 & 4 & 4 & 2 & 0 \\
\hline $2 \mathrm{~A}$ & 7 & 3 & 1 & 3 & 0 & 2 & 2 & 1 & 2 \\
\hline $2 \mathrm{~B}$ & 5 & 1 & 0 & 0 & 1 & 3 & 3 & 0 & 2 \\
\hline $2 \mathrm{C}$ & 6 & 1 & 1 & 1 & 0 & 1 & 0 & 0 & 4 \\
\hline $2 \mathrm{D}$ & 7 & 3 & 3 & 5 & 2 & 4 & 3 & 2 & 0 \\
\hline $2 \mathrm{E}$ & 6 & 3 & 3 & 2 & 1 & 2 & 4 & 2 & 2 \\
\hline $3 \mathrm{~B}$ & 9 & 7 & 3 & 5 & 1 & 3 & 4 & 0 & 1 \\
\hline $3 C$ & 7 & 3 & 3 & 4 & 1 & 2 & 4 & 1 & 2 \\
\hline $3 \mathrm{D}$ & 6 & 1 & 3 & 1 & 0 & 2 & 2 & 0 & 3 \\
\hline $3 \mathrm{E}$ & 7 & 2 & 4 & 2 & 2 & 3 & 4 & 1 & 2 \\
\hline $4 \mathrm{~B}$ & 6 & 5 & 4 & 4 & 5 & 4 & 2 & 4 & 1 \\
\hline $4 \mathrm{C}$ & 9 & 3 & 2 & 5 & 0 & 5 & 0 & 1 & 1 \\
\hline $4 \mathrm{D}$ & 10 & 4 & 4 & 3 & 3 & 6 & 2 & 3 & 3 \\
\hline $4 \mathrm{E}$ & 10 & 3 & 4 & 2 & 2 & 4 & 2 & 1 & 5 \\
\hline $5 \mathrm{~B}$ & 2 & 1 & 0 & 1 & 1 & 0 & 1 & 0 & 1 \\
\hline $5 \mathrm{C}$ & 8 & 3 & 2 & 4 & 1 & 0 & 2 & 1 & 3 \\
\hline $5 \mathrm{D}$ & 3 & 1 & 1 & 1 & 1 & 2 & 1 & 0 & 1 \\
\hline $5 \mathrm{E}$ & 4 & 0 & 0 & 1 & 0 & 2 & 1 & 1 & 1 \\
\hline Totals & 139 & 56 & 43 & 59 & 31 & 60 & 52 & 20 & 37 \\
\hline
\end{tabular}

\section{Target Location Performance}

In order to evaluate the demonstrator's field survey data, a computer program was compiled in SAS software. This program calculated an evaluation variable by sequentially going from the top of the prioritized dig list and determining if each object on the list (whether classified as target or clutter) corresponds to an emplaced target location within $0.5 \mathrm{~m}$ (a detection) or not (a false alarm). Similarly, Appendix C considers a detection if it is within $1.0 \mathrm{~m}$.

The location (x,y) performance (Table 13) of each of the seven demonstrators was evaluated by comparing each item in the dig list with the groundtruth, determining the closest item to an emplaced UXO target location, and computing the error. The ability to locate clutter items was not one of the evaluation criteria, but the raw data were available for such analysis if deemed useful. 


\begin{tabular}{|c|c|c|c|c|c|c|c|c|}
\hline \multicolumn{9}{|c|}{$\begin{array}{l}\text { Table } 5 \\
\text { UXO Found - NAEVA }\end{array}$} \\
\hline \multirow[b]{3}{*}{ Description } & \multicolumn{8}{|c|}{ Area } \\
\hline & \multicolumn{4}{|c|}{ Actual Targets } & \multicolumn{4}{|c|}{ Within $0.5 \mathrm{~m}$} \\
\hline & $\mathbf{A}$ & B & C & Total & A & B & C & Total \\
\hline 2.25-in. & 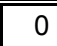 & 3 & 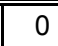 & 3 & 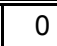 & 2 & 0 & 2 \\
\hline 2.75-in. & 4 & 5 & 4 & 13 & 4 & 1 & 1 & 6 \\
\hline 5-in. & 2 & 4 & 2 & 8 & 0 & 2 & 0 & 2 \\
\hline $20-\mathrm{mm}$ & 4 & 12 & 1 & 17 & 1 & 0 & 0 & 1 \\
\hline 40-mm & 1 & \begin{tabular}{|l|}
14 \\
\end{tabular} & 5 & 20 & 1 & 7 & 1 & 9 \\
\hline 60-mm & 2 & 11 & 3 & 16 & 2 & 5 & 1 & 8 \\
\hline 81-mm & 1 & 8 & 3 & 12 & 1 & 5 & 1 & 7 \\
\hline 105-mm & 1 & 3 & 1 & 5 & 0 & 2 & 1 & 3 \\
\hline BDU-33 & 3 & 4 & 3 & 10 & 2 & 2 & 2 & 6 \\
\hline Mk-3 & 1 & 4 & 3 & 8 & 1 & 3 & 2 & 6 \\
\hline Mk-81 & 1 & 2 & 1 & 4 & 0 & 0 & 0 & 0 \\
\hline Mk-82 & 1 & 4 & 7 & 12 & 0 & 2 & 1 & 3 \\
\hline Mk-83 & 0 & 1 & 0 & 1 & 0 & 1 & 0 & 1 \\
\hline Mk-106 & 0 & 4 & 0 & 4 & 0 & 2 & 0 & 2 \\
\hline LAAW & 2 & 1 & 0 & 3 & 1 & 0 & 0 & 1 \\
\hline SMAW & 1 & 1 & 1 & 3 & 0 & 0 & 0 & 0 \\
\hline Total & 24 & 81 & 34 & 139 & 13 & 33 & 10 & 56 \\
\hline
\end{tabular}

\begin{tabular}{|c|c|c|c|c|c|c|c|c|}
\hline \multicolumn{9}{|c|}{$\begin{array}{l}\text { Table } 6 \\
\text { UXO Found - GTL }\end{array}$} \\
\hline \multirow[b]{3}{*}{ Description } & \multicolumn{8}{|c|}{ Area } \\
\hline & \multicolumn{4}{|c|}{ Actual Targets } & \multicolumn{4}{|c|}{ Within $0.5 \mathrm{~m}$} \\
\hline & A & B & C & Total & A & B & C & Total \\
\hline 2.25-in. & 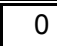 & 3 & 0 & 3 & 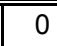 & 2 & 0 & 2 \\
\hline 2.75-in. & 4 & 5 & 4 & 13 & 2 & 0 & 1 & 3 \\
\hline 5-in. & 2 & 4 & 2 & 8 & 0 & 3 & 0 & 3 \\
\hline $20-\mathrm{mm}$ & 4 & 12 & 1 & 17 & 1 & 1 & 0 & 2 \\
\hline 40-mm & 1 & 14 & 5 & 20 & 0 & 6 & 0 & 6 \\
\hline 60-mm & 2 & 11 & 3 & 16 & 2 & 4 & 1 & 7 \\
\hline $81-\mathrm{mm}$ & 1 & 8 & 3 & 12 & 1 & 5 & 1 & 7 \\
\hline $105-\mathrm{mm}$ & 1 & 3 & 1 & 5 & 1 & 1 & 0 & 2 \\
\hline BDU-33 & 3 & 4 & 3 & 10 & 2 & 1 & 0 & 3 \\
\hline Mk-3 & 1 & 4 & 3 & 8 & 0 & 2 & 0 & 2 \\
\hline Mk-81 & 1 & 2 & 1 & 4 & 0 & 0 & 0 & 0 \\
\hline Mk-82 & 1 & 4 & 7 & 12 & 0 & 2 & 0 & 2 \\
\hline Mk-83 & 0 & 1 & 0 & 1 & 0 & 1 & 0 & 1 \\
\hline Mk-106 & 0 & 4 & 0 & 4 & 0 & 3 & 0 & 3 \\
\hline LAAW & 2 & 1 & 0 & 3 & 1 & 0 & 0 & 1 \\
\hline SMAW & 1 & 1 & 1 & 3 & 0 & 0 & 0 & 0 \\
\hline Total & 24 & 81 & 34 & 139 & 10 & 30 & 3 & 43 \\
\hline
\end{tabular}




\begin{tabular}{|c|c|c|c|c|c|c|c|c|}
\hline \multicolumn{9}{|c|}{$\begin{array}{l}\text { Table } 7 \\
\text { UXO Found - Geophex \& Geophex without } 20 / 40 \text { mm }\end{array}$} \\
\hline \multirow[b]{3}{*}{ Description } & \multicolumn{8}{|c|}{ Area } \\
\hline & \multicolumn{4}{|c|}{ Actual Targets } & \multicolumn{4}{|c|}{ Within $0.5 \mathrm{~m}$} \\
\hline & A & B & C & Total & A & $\mathrm{B}$ & C & Total \\
\hline 2.25-in. & 0 & 3 & 0 & 3 & 0 & 2 & 0 & 2 \\
\hline 2.75-in. & 4 & 5 & 4 & 13 & 2 & 1 & 1 & 4 \\
\hline 5-in. & 2 & 4 & 2 & 8 & 1 & 3 & 0 & 4 \\
\hline $20-\mathrm{mm}$ & 4 & 12 & 1 & 17 & 2 & 1 & 0 & 3 \\
\hline 40-mm & 1 & 14 & 5 & 20 & 1 & 4 & 1 & 6 \\
\hline 60-mm & 2 & 11 & 3 & 16 & 1 & 3 & 2 & 6 \\
\hline 81-mm & 1 & 8 & 3 & 12 & 1 & 6 & 1 & 8 \\
\hline 105-mm & 1 & 3 & 1 & 5 & 1 & 3 & 0 & 4 \\
\hline BDU-33 & 3 & 4 & 3 & 10 & 3 & 2 & 2 & 7 \\
\hline Mk-3 & 1 & 4 & 3 & 8 & 1 & 3 & 2 & 6 \\
\hline Mk-81 & 1 & 2 & 1 & 4 & 1 & 0 & 0 & 1 \\
\hline Mk-82 & 1 & 4 & 7 & 12 & 0 & 2 & 1 & 3 \\
\hline Mk-83 & 0 & 1 & 0 & 1 & 0 & 1 & 0 & 1 \\
\hline Mk-106 & 0 & 4 & 0 & 4 & 0 & 3 & 0 & 3 \\
\hline LAAW & 2 & 1 & 0 & 3 & 1 & 0 & 0 & 1 \\
\hline SMAW & 1 & 1 & 1 & 3 & 1 & 0 & 0 & 1 \\
\hline Total & 24 & 81 & 34 & 139 & 16 & 33 & 10 & 59 \\
\hline
\end{tabular}

\begin{tabular}{|c|c|c|c|c|c|c|c|c|}
\hline \multicolumn{9}{|c|}{$\begin{array}{l}\text { Table } 8 \\
\text { UXO Found - NRL }\end{array}$} \\
\hline \multirow[b]{3}{*}{ Description } & \multicolumn{8}{|c|}{ Area } \\
\hline & \multicolumn{4}{|c|}{ Actual Targets } & \multicolumn{4}{|c|}{ Within $0.5 \mathrm{~m}$} \\
\hline & A & B & C & Total & A & B & C & Total \\
\hline 2.25-in. & 0 & 3 & 0 & 3 & 0 & 2 & 0 & 2 \\
\hline 2.75-in. & 4 & 5 & 4 & 13 & 1 & 1 & 1 & 3 \\
\hline 5-in. & 2 & 4 & 2 & 8 & 1 & 2 & 0 & 3 \\
\hline 20-mm & 4 & 12 & 1 & 17 & 1 & 0 & 0 & 1 \\
\hline 40-mm & 1 & 14 & 5 & 20 & 0 & 0 & 0 & 0 \\
\hline 60-mm & 2 & 11 & 3 & 16 & 1 & 2 & 1 & 4 \\
\hline $81-\mathrm{mm}$ & 1 & 8 & 3 & 12 & 0 & 3 & 0 & 3 \\
\hline 105-mm & 1 & 3 & 1 & 5 & 0 & 2 & 0 & 2 \\
\hline BDU-33 & 3 & 4 & 3 & 10 & 3 & 1 & 1 & 5 \\
\hline Mk-3 & 1 & 4 & 3 & 8 & 0 & 1 & 0 & 1 \\
\hline Mk-81 & 1 & 2 & 1 & 4 & 0 & 0 & 1 & 1 \\
\hline Mk-82 & 1 & 4 & 7 & 12 & 0 & 1 & 2 & 3 \\
\hline Mk-83 & 0 & 1 & 0 & 1 & 0 & 1 & 0 & 1 \\
\hline Mk-106 & 0 & 4 & 0 & 4 & 0 & 2 & 0 & 2 \\
\hline LAAW & 2 & 1 & 0 & 3 & 1 & 0 & 0 & 1 \\
\hline SMAW & 1 & 1 & 1 & 3 & 0 & 0 & 0 & 0 \\
\hline Total & 24 & 81 & 34 & 139 & 8 & 17 & 6 & 31 \\
\hline
\end{tabular}




\begin{tabular}{|c|c|c|c|c|c|c|c|c|}
\hline \multicolumn{9}{|c|}{$\begin{array}{l}\text { Table } 9 \\
\text { UXO Found - NRL without } 20 / 40 \mathrm{~mm}\end{array}$} \\
\hline \multirow[b]{3}{*}{ Description } & \multicolumn{8}{|c|}{ Area } \\
\hline & \multicolumn{4}{|c|}{ Actual Targets } & \multicolumn{4}{|c|}{ Within $0.5 \mathrm{~m}$} \\
\hline & A & B & C & Total & A & B & C & Total \\
\hline 2.25-in. & 0 & 3 & 0 & 3 & 0 & 2 & 0 & 2 \\
\hline 2.75-in. & 4 & 5 & 4 & 13 & 1 & 1 & 1 & 3 \\
\hline 5-in. & 2 & 4 & 2 & 8 & 1 & 2 & 0 & 3 \\
\hline $20-\mathrm{mm}$ & 4 & 12 & 1 & 17 & 1 & 0 & 0 & 1 \\
\hline 40-mm & 1 & 14 & 5 & 20 & 0 & 0 & 0 & 0 \\
\hline 60-mm & 2 & 11 & 3 & 16 & 2 & 3 & 1 & 6 \\
\hline 81-mm & 1 & 8 & 3 & 12 & 0 & 3 & 1 & 4 \\
\hline $105-\mathrm{mm}$ & 1 & 3 & 1 & 5 & 0 & 2 & 0 & 2 \\
\hline BDU-33 & 3 & 4 & 3 & 10 & 3 & 1 & 1 & 5 \\
\hline Mk-3 & 1 & 4 & 3 & 8 & 0 & 2 & 0 & 2 \\
\hline Mk-81 & 1 & 2 & 1 & 4 & 0 & 0 & 1 & 1 \\
\hline \begin{tabular}{|l|} 
Mk-82 \\
\end{tabular} & 1 & 4 & 7 & 12 & 0 & 1 & 2 & 3 \\
\hline Mk-83 & 0 & 1 & 0 & 1 & 0 & 1 & 0 & 1 \\
\hline Mk-106 & 0 & 4 & 0 & 4 & 0 & 2 & 0 & 2 \\
\hline LAAW & 2 & 1 & 0 & 3 & 1 & 0 & 0 & 1 \\
\hline SMAW & 1 & 1 & 1 & 3 & 0 & 0 & 1 & 1 \\
\hline \begin{tabular}{|l} 
Total \\
\end{tabular} & 24 & 81 & 34 & 139 & 9 & 19 & 8 & 36 \\
\hline
\end{tabular}

\begin{tabular}{|c|c|c|c|c|c|c|c|c|}
\hline \multicolumn{9}{|c|}{\begin{tabular}{|l|l|} 
Table 10 \\
UXO Found - Parsons EM-61 EM \& Flag \\
\end{tabular}} \\
\hline \multirow[b]{3}{*}{ Description } & \multicolumn{8}{|c|}{ Area } \\
\hline & \multicolumn{4}{|c|}{ Actual Targets } & \multicolumn{4}{|c|}{ Within $0.5 \mathrm{~m}$} \\
\hline & A & B & C & Total & A & B & C & Total \\
\hline 2.25-in. & 0 & 3 & 0 & 3 & 0 & 2 & 0 & 2 \\
\hline 2.75-in. & 4 & 5 & 4 & 13 & 2 & 2 & 1 & 5 \\
\hline 5-in. & 2 & 4 & 2 & 8 & 2 & 3 & 1 & 6 \\
\hline $20-\mathrm{mm}$ & 4 & 12 & 1 & 17 & 0 & 1 & 0 & 1 \\
\hline 40-mm & 1 & 14 & 5 & 20 & 1 & 6 & 0 & 7 \\
\hline 60-mm & 2 & 11 & 3 & 16 & 0 & 4 & 2 & 6 \\
\hline 81-mm & 1 & 8 & 3 & 12 & 1 & 3 & 2 & 6 \\
\hline 105-mm & 1 & 3 & 1 & 5 & 1 & 2 & 1 & 4 \\
\hline BDU-33 & 3 & 4 & 3 & 10 & 3 & 2 & 2 & 7 \\
\hline $\mid$ Mk-3 & 1 & 4 & 3 & 8 & 1 & 3 & 1 & 5 \\
\hline Mk-81 & 1 & 2 & 1 & 4 & 0 & 1 & 0 & 1 \\
\hline Mk-82 & 1 & 4 & 7 & 12 & 1 & 1 & 4 & 6 \\
\hline Mk-83 & 0 & 1 & 0 & 1 & 0 & 1 & 0 & 1 \\
\hline Mk-106 & 0 & 4 & 0 & 4 & 0 & 3 & 0 & 3 \\
\hline LAAW & 2 & 1 & 0 & 3 & 0 & 0 & 0 & 0 \\
\hline SMAW & 1 & 1 & 1 & 3 & 0 & 0 & 1 & 1 \\
\hline |Total & 24 & 81 & 34 & 139 & 12 & 33 & 15 & 60 \\
\hline
\end{tabular}




\begin{tabular}{|c|c|c|c|c|c|c|c|c|}
\hline \multicolumn{9}{|c|}{$\begin{array}{l}\text { Table } 11 \\
\text { UXO Found - Parsons EM-61 Digital }\end{array}$} \\
\hline \multirow[b]{3}{*}{ Description } & \multicolumn{8}{|c|}{ Area } \\
\hline & \multicolumn{4}{|c|}{ Actual Targets } & \multicolumn{4}{|c|}{ Within $0.5 \mathrm{~m}$} \\
\hline & A & B & C & Total & A & B & C & Total \\
\hline 2.25-in. & 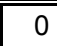 & 3 & 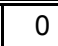 & 3 & 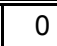 & 1 & 0 & 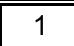 \\
\hline \begin{tabular}{|l}
2.75 -in. \\
\end{tabular} & 4 & 5 & 4 & 13 & 1 & 1 & 1 & 3 \\
\hline 5-in. & 2 & 4 & 2 & 8 & 2 & 3 & 1 & 6 \\
\hline $20-\mathrm{mm}$ & 4 & 12 & 1 & 17 & 1 & 0 & 0 & 1 \\
\hline 40-mm & 1 & 14 & 5 & 20 & 1 & 1 & 0 & 2 \\
\hline 60-mm & 2 & 11 & 3 & 16 & 1 & 5 & 1 & 7 \\
\hline 81-mm & 1 & 8 & 3 & 12 & 1 & 6 & 1 & 8 \\
\hline \begin{tabular}{|l|}
$105-\mathrm{mm}$ \\
\end{tabular} & 1 & 3 & 1 & 5 & 1 & 2 & 1 & 4 \\
\hline BDU-33 & 3 & 4 & 3 & 10 & 2 & 1 & 2 & 5 \\
\hline Mk-3 & 1 & 4 & 3 & 8 & 0 & 1 & 3 & 4 \\
\hline Mk-81 & 1 & 2 & 1 & 4 & 1 & 1 & 0 & 2 \\
\hline Mk-82 & 1 & 4 & 7 & 12 & 0 & 1 & 4 & 5 \\
\hline Mk-83 & 0 & 1 & 0 & 1 & 0 & 1 & 0 & 1 \\
\hline Mk-106 & 0 & 4 & 0 & 4 & 0 & 2 & 0 & 2 \\
\hline LAAW & 2 & 1 & 0 & 3 & 1 & 0 & 0 & 1 \\
\hline SMAW & 1 & 1 & 1 & 3 & 0 & 0 & 1 & 1 \\
\hline Total & 24 & 81 & 34 & 139 & 12 & 25 & 15 & 52 \\
\hline
\end{tabular}

\begin{tabular}{|c|c|c|c|c|c|c|c|c|}
\hline $\begin{array}{l}\text { Table } 12 \\
\text { UXO Fou }\end{array}$ & d - & 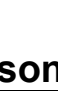 & 1-6 & $-5 E$ & & & & \\
\hline & & & & & rea & & & \\
\hline & & & Targ & & & & 0.5 & \\
\hline Description & A & $B$ & C & Total & A & B & C & Total \\
\hline 2.25-in. & 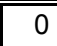 & 3 & 0 & 3 & 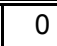 & 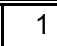 & 0 & 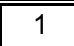 \\
\hline 2.75-in. & 4 & 5 & 4 & 13 & 0 & 2 & 0 & 2 \\
\hline 5-in. & 2 & 4 & 2 & 8 & 0 & 1 & 0 & 1 \\
\hline $20-\mathrm{mm}$ & 4 & 12 & 1 & 17 & 0 & 0 & 0 & 0 \\
\hline 40-mm & 1 & 14 & 5 & 20 & 0 & 1 & 0 & 1 \\
\hline 60-mm & 2 & 11 & 3 & 16 & 1 & 3 & 0 & 4 \\
\hline $81-\mathrm{mm}$ & 1 & 8 & 3 & 12 & 1 & 0 & 1 & 2 \\
\hline 105-mm & 1 & 3 & 1 & 5 & 1 & 1 & 0 & 2 \\
\hline BDU-33 & 3 & 4 & 3 & 10 & 0 & 0 & 0 & 0 \\
\hline Mk-3 & 1 & 4 & 3 & 8 & 3 & 1 & 0 & 4 \\
\hline Mk-81 & 1 & 2 & 1 & 4 & 0 & 1 & 0 & 1 \\
\hline Mk-82 & 1 & 4 & 7 & 12 & 0 & 1 & 0 & 1 \\
\hline Mk-83 & 0 & 1 & 0 & 1 & 0 & 1 & 0 & 1 \\
\hline Mk-106 & 0 & 4 & 0 & 4 & 0 & 0 & 0 & 0 \\
\hline \begin{tabular}{|l|} 
LAAW \\
\end{tabular} & 2 & 1 & 0 & 3 & 0 & 1 & 0 & 1 \\
\hline SMAW & 1 & 1 & 1 & 3 & 0 & 0 & 0 & 0 \\
\hline Total & 24 & 81 & 34 & 139 & 6 & 13 & 1 & 20 \\
\hline
\end{tabular}




\begin{tabular}{|c|c|c|c|c|c|}
\hline \multicolumn{6}{|c|}{$\begin{array}{l}\text { Table } 13 \\
\text { UXO Target Location }(x, y) \text { Estimation Performance of the } \\
\text { Demonstrators }\end{array}$} \\
\hline Area & Demonstrator & \begin{tabular}{|l|} 
Minimum \\
Error, $\mathrm{m}$ \\
\end{tabular} & \begin{tabular}{|l|} 
Maximum \\
Error, $\mathrm{m}$
\end{tabular} & $\begin{array}{l}\text { Mean Error, } \\
\text { m }\end{array}$ & \begin{tabular}{|l} 
Standard \\
Deviation
\end{tabular} \\
\hline \multirow[t]{8}{*}{ A } & $\overline{\text { NAEVA }}$ & 0.065 & 0.968 & 0.444 & 0.233 \\
\hline & GTL & 0.139 & 0.856 & 0.395 & 0.249 \\
\hline & Geophex & 0.105 & 0.941 & 0.357 & 0.217 \\
\hline & NRL & 0.146 & 0.842 & 0.395 & 0.213 \\
\hline & NRL without $20 / 40 \mathrm{~mm}$ & 0.123 & 0.911 & 0.406 & 0.271 \\
\hline & Parson (EM-61) EM and Flag & 0.074 & 0.737 & 0.403 & 0.198 \\
\hline & Parson EM-61 Digital & 0.020 & 0.993 & 0.513 & 0.270 \\
\hline & Parson TM-5 EMU & 0.108 & 0.534 & 0.322 & 0.161 \\
\hline \multirow[t]{8}{*}{$B$} & NAEVA & 0.071 & 0.960 & 0.444 & 0.232 \\
\hline & GTL & 0.036 & 0.877 & 0.400 & 0.258 \\
\hline & Geophex & 0.025 & 0.984 & 0.403 & 0.246 \\
\hline & NRL & 0.080 & 0.979 & 0.445 & 0.279 \\
\hline & NRL without $20 / 40 \mathrm{~mm}$ & 0.080 & 0.999 & 0.530 & 0.289 \\
\hline & Parson (EM-61) EM and Flag & 0.040 & 0.991 & 0.394 & 0.247 \\
\hline & Parson EM-61 Digital & 0.028 & 0.970 & 0.435 & 0.259 \\
\hline & Parson TM-5 EMU & 0.200 & 0.886 & 0.629 & 0.302 \\
\hline \multirow[t]{8}{*}{ C } & NAEVA & 0.074 & 0.992 & 0.505 & 0.302 \\
\hline & GTL & 0.260 & 0.981 & 0.673 & 0.203 \\
\hline & Geophex & 0.114 & 0.820 & 0.394 & 0.244 \\
\hline & NRL & 0.234 & 0.718 & 0.434 & 0.199 \\
\hline & NRL without $20 / 40 \mathrm{~mm}$ & 0.234 & 0.771 & 0.461 & 0.193 \\
\hline & Parson (EM-61) EM and Flag & 0.064 & 0.876 & 0.405 & 0.225 \\
\hline & Parson EM-61 Digital & 0.074 & 0.863 & 0.419 & 0.225 \\
\hline & Parson TM-5 EMU & 0.089 & 0.914 & 0.438 & 0.273 \\
\hline
\end{tabular}

The ability of the demonstrators to estimate the depth of the UXO targets is summarized in Table 14. These results indicate that, while the performance of each demonstrator varied significantly between each demonstration area, the mean depth estimation errors were within the $0.5-\mathrm{m}$ allowable error. Overall, the PARSON TM-5 EMU system achieved the best depth estimation accuracy for Areas A and C. The accuracy was the worst in Area B, with the largest maximum and mean depth estimation errors demonstrated. The ranking of the demonstrators differed in each area.

\section{Discrimination Results}

Tables 15 through 30 illustrate the comparison of the demonstrator's declarations versus the groundtruth emplaced targets. Table 15 summarizes the performance of the demonstrators over the entire demonstration site within the QA Range. In this table, the ordnance items left in the ground represent the items declared by the demonstrator as clutter with high confidence that are actually groundtruth emplaced ordnance plus the number of undetected ordnance items. The variable Correct Discrimination is the total of the correctly identified ordnance items. The False Alarm number is the total of the other detections plus the total of the Groundtruth Fragment Matches minus the number of objects identified as Clutter with High confidence. 


\begin{tabular}{|c|c|c|c|c|c|}
\hline \multicolumn{6}{|c|}{$\begin{array}{l}\text { Table } 14 \\
\text { UXO Target Depth Estimation Performance of the Demonstrators }\end{array}$} \\
\hline Area & Demonstrator & \begin{tabular}{|l} 
Minimum \\
Error, $\mathrm{m}$
\end{tabular} & \begin{tabular}{|l} 
Maximum \\
Error, $\mathrm{m}$
\end{tabular} & \begin{tabular}{|l} 
Mean Error, \\
m
\end{tabular} & $\begin{array}{l}\text { Standard } \\
\text { Deviation }\end{array}$ \\
\hline \multirow[t]{7}{*}{ 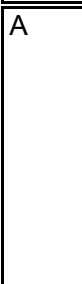 } & Naeva & 0.010 & 0.394 & 0.233 & 0.118 \\
\hline & GTL & 0.000 & 0.970 & 0.208 & 0.198 \\
\hline & Geophex & 0.000 & 1.250 & 0.278 & 0.244 \\
\hline & NRL & 0.015 & 0.677 & 0.360 & 0.180 \\
\hline & Parson (EM-61) EM and Flag & 0.000 & 1.020 & 0.232 & 0.237 \\
\hline & Parson EM-61 Digital & 0.020 & 0.550 & 0.219 & 0.128 \\
\hline & Parson TM-5 EMU & & & & \\
\hline \multirow[t]{7}{*}{$B$} & Naeva & 0.006 & 1.392 & 0.218 & 0.188 \\
\hline & GTL & 0.004 & 0.560 & 0.184 & 0.140 \\
\hline & Geophex & 0.000 & 1.150 & 0.286 & 0.255 \\
\hline & NRL & 0.015 & 0.830 & 0.370 & 0.204 \\
\hline & Parson (EM-61) EM and Flag & 0.000 & 1.832 & 0.212 & 0.261 \\
\hline & Parson EM-61 Digital & 0.010 & 0.570 & 02138 & 0.131 \\
\hline & Parson TM-5 EMU & & & & \\
\hline \multirow[t]{7}{*}{ C } & Naeva & 0.010 & 0.552 & 0.207 & 0.134 \\
\hline & GTL & 0.010 & 0.921 & 0.217 & 0.211 \\
\hline & Geophex & 0.000 & 1.274 & 0.223 & 0.234 \\
\hline & NRL & 0.039 & 0.652 & 0.313 & 0.187 \\
\hline & Parson (EM-61) EM and Flag & 0.000 & 1.324 & 0.233 & 0.267 \\
\hline & Parson EM-61 Digital & 0.000 & 0.840 & 0.202 & 0.132 \\
\hline & Parson TM-5 EMU & & & & \\
\hline
\end{tabular}

Tables 16 through 30 show more detailed breakdowns of the results by area for each demonstrator. The left most column of each of these tables represents the emplaced targets. These items are matched with the demonstrator's dig list. When the demonstrator reported the object as ordnance and the emplaced target was a groundtruth ordnance match then it is considered a correct discrimination. The confidence of the demonstrator's declaration is used to determine which items from his dig list are to be excavated. In lieu of a demonstrators' defined "stop dig point," only objects that are declared as clutter with high confidence would be left in the ground.

For example, NAEVA (under $0.5 \mathrm{~m}$ ) discrimination performance is illustrated in Table 16. NAEVA reported 295 items as Ordnance. Of that number, 37 actually matched the ground-truth ordnance items emplaced in the ground, 34 matched the ground-truth-emplaced fragments, and 224 detected something other than the targets emplaced in the ground. NAEVA reported 630 items as clutter. Only 25 actually matched the Groundtruth Fragments emplaced in the ground, 19 matched the groundtruth emplaced ordnance items, and 586 detected something other than the targets emplaced in the ground. NAEVA reported 250 $(241+4+5)$ with high confidence of the 630 clutter items, therefore, 40 percent of the identified clutter were designated as "do not dig." There were five ordnance items that would have been left in the ground as a result of being classified as high confidence clutter. Also, from Table 15, there were 88 undetected ordnance items. NAEVA reported a total of 810 items which did not match any emplaced ordnance, and 59 items that matched emplaced fragments and 56 detections of emplaced ordnances. 


\begin{tabular}{|c|c|c|c|c|c|}
\hline \multicolumn{6}{|c|}{$\begin{array}{l}\text { Table } 15 \\
\text { Summary of Discrimination Performance }\end{array}$} \\
\hline & \multicolumn{2}{|c|}{$\begin{array}{c}\text { Ordnance Left in the } \\
\text { Ground }\end{array}$} & \multicolumn{2}{|c|}{ Correct Discrimination } & $\begin{array}{c}\begin{array}{c}\text { False Alarm } \\
\text { Number }\end{array} \\
\end{array}$ \\
\hline & \multicolumn{2}{|c|}{ Within $0.5 \mathrm{~m}$} & \multicolumn{2}{|c|}{ Within $0.5 \mathrm{~m}$} & Within $0.5 \mathrm{~m}$ \\
\hline & Num & Percent & Num & Percent & \\
\hline Naeva & 88 & $63.31 \%$ & 51 & $36.69 \%$ & 624 \\
\hline GTL & 96 & $69.06 \%$ & 43 & $30.94 \%$ & 1,283 \\
\hline Geophex & 80 & $57.55 \%$ & 59 & $42.45 \%$ & 772 \\
\hline Geophex without $20 / 40 \mathrm{~mm}$ & 80 & $57.55 \%$ & 59 & $42.45 \%$ & 772 \\
\hline $\mathrm{NRL}$ & 108 & $77.70 \%$ & 31 & $22.30 \%$ & 342 \\
\hline NRL without $20 / 40 \mathrm{~mm}$ & 102 & $73.38 \%$ & 37 & $26.62 \%$ & 602 \\
\hline Parson (EM-61) EM \& Flag & 79 & $56.83 \%$ & 60 & $43.17 \%$ & 872 \\
\hline Parson EM-61 Digital & 87 & $62.59 \%$ & 52 & $37.41 \%$ & 1,405 \\
\hline Parson TM-5 EMU & 119 & $85.61 \%$ & 20 & $14.39 \%$ & 172 \\
\hline
\end{tabular}

\begin{tabular}{|c|c|c|c|c|c|c|c|}
\hline \multicolumn{8}{|c|}{$\begin{array}{l}\text { Table } 16 \\
\text { Breakdown of Discrimination - QA Range and Area A - NAEVA }\end{array}$} \\
\hline \multirow[b]{3}{*}{$\begin{array}{l}\text { Ground- } \\
\text { truth }\end{array}$} & \multicolumn{7}{|c|}{ Demonstrator Classification } \\
\hline & \multirow[b]{2}{*}{ Confidence } & \multicolumn{3}{|c|}{ QA Range } & \multicolumn{3}{|c|}{ Area A } \\
\hline & & \begin{tabular}{|l|} 
Total \\
Anomalies
\end{tabular} & $\begin{array}{l}\text { Declared } \\
\text { Ordnance }\end{array}$ & $\begin{array}{l}\text { Declared } \\
\text { Clutter }\end{array}$ & \begin{tabular}{|l|} 
Total \\
Anomalies
\end{tabular} & $\begin{array}{l}\text { Declared } \\
\text { Ordnance }\end{array}$ & $\begin{array}{l}\text { Declared } \\
\text { Clutter }\end{array}$ \\
\hline \multirow{3}{*}{$\begin{array}{l}\text { Non- } \\
\text { emplaced }\end{array}$} & High & 381 & 140 & 241 & 171 & 31 & 140 \\
\hline & Low & 429 & 84 & 345 & 99 & 23 & 76 \\
\hline & Total & 810 & 224 & 586 & 270 & 54 & 216 \\
\hline \multirow[t]{3}{*}{ Clutter } & High & 30 & 26 & 4 & 9 & 6 & 3 \\
\hline & Low & 29 & 8 & 21 & 7 & 2 & 5 \\
\hline & Total & 59 & 34 & 25 & 16 & 8 & 8 \\
\hline \multirow[t]{3}{*}{ Ordnance } & High & 31 & 26 & 5 & 8 & 6 & 2 \\
\hline & Low & 25 & 11 & 14 & 5 & 1 & 4 \\
\hline & Total & 56 & 37 & 19 & 13 & 7 & 6 \\
\hline Total & & 925 & 295 & 630 & 299 & 69 & 230 \\
\hline
\end{tabular}

\begin{tabular}{|c|c|c|c|c|c|c|c|}
\hline $\begin{array}{l}\text { Table } 1 \\
\text { Breakd }\end{array}$ & wn of Di & criminati & on - Are & a B and & Area C - & NAEVA & \\
\hline & & & Demonst & ator Class & ification & & \\
\hline & & & Area B & & & Area C & \\
\hline $\begin{array}{l}\text { Ground- } \\
\text { truth }\end{array}$ & Confidence & \begin{tabular}{|l|} 
Total \\
Anomalies
\end{tabular} & $\begin{array}{l}\text { Declared } \\
\text { Ordnance }\end{array}$ & $\begin{array}{l}\text { Declared } \\
\text { Clutter }\end{array}$ & $\begin{array}{l}\text { Total } \\
\text { Anomalies }\end{array}$ & $\begin{array}{l}\text { Declared } \\
\text { Ordnance }\end{array}$ & $\begin{array}{l}\text { Declared } \\
\text { Clutter }\end{array}$ \\
\hline Non- & High & 127 & 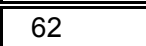 & 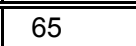 & 83 & 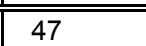 & 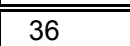 \\
\hline emplaced & Low & 185 & 32 & 153 & 145 & 29 & 116 \\
\hline & \begin{tabular}{|l|} 
Total \\
\end{tabular} & 312 & 94 & 218 & 228 & 76 & 152 \\
\hline Clutter & High & 14 & 13 & 1 & 7 & 7 & 0 \\
\hline & Low & 13 & 2 & 11 & 9 & 4 & 5 \\
\hline & \begin{tabular}{|l|} 
Total \\
\end{tabular} & 27 & 15 & 12 & 16 & 11 & 5 \\
\hline Ordnance & High & 17 & 15 & 2 & 6 & 5 & 1 \\
\hline & Low & 16 & 8 & 8 & 4 & 2 & 2 \\
\hline & Total & 33 & 23 & 10 & 10 & 7 & 3 \\
\hline Total & & 372 & 132 & 240 & 254 & 94 & 160 \\
\hline
\end{tabular}




\begin{tabular}{|c|c|c|c|c|c|}
\hline \multicolumn{6}{|c|}{\begin{tabular}{|l} 
Table 18 \\
Breakdown of Discrimination - QA Range - GTL
\end{tabular}} \\
\hline \multirow[b]{3}{*}{$\begin{array}{l}\text { Ground- } \\
\text { truth }\end{array}$} & \multicolumn{5}{|c|}{ Demonstrator Classification } \\
\hline & & Total & Area A & Area B & Area C \\
\hline & Confidence & \begin{tabular}{|l|} 
Declared \\
Ordnance \\
\end{tabular} & \begin{tabular}{|l|} 
Declared \\
Ordnance \\
\end{tabular} & \begin{tabular}{|l|} 
Declared \\
Ordnance \\
\end{tabular} & \begin{tabular}{|l|} 
Declared \\
Ordnance \\
\end{tabular} \\
\hline \multirow{4}{*}{\begin{tabular}{|l|} 
Non- \\
emplaced
\end{tabular}} & High & 682 & 96 & 324 & 262 \\
\hline & Low & 307 & 66 & 167 & 74 \\
\hline & Medium & 234 & 56 & 122 & 56 \\
\hline & Total & 1,223 & 218 & 613 & 392 \\
\hline \multirow[t]{4}{*}{ Clutter } & High & 46 & 13 & 23 & 10 \\
\hline & Low & 8 & 2 & 4 & 2 \\
\hline & Medium & 6 & 3 & 2 & 1 \\
\hline & Total & 60 & 18 & 29 & 13 \\
\hline \multirow[t]{4}{*}{ Ordnance } & High & 35 & 8 & 25 & 2 \\
\hline & Low & 3 & 1 & 1 & 1 \\
\hline & Medium & 5 & 1 & 4 & 0 \\
\hline & \begin{tabular}{|l|} 
Total \\
\end{tabular} & 43 & 10 & 30 & 3 \\
\hline Total & & 1,326 & 246 & 672 & 408 \\
\hline
\end{tabular}

\begin{tabular}{|c|c|c|c|c|c|c|c|}
\hline \multicolumn{8}{|c|}{$\begin{array}{l}\text { Table } 19 \\
\text { Breakdown of Discrimination - QA Range and Area A- Geophex \& } \\
\text { Geophex without } 20 / 40 \mathrm{~mm}\end{array}$} \\
\hline \multirow[b]{3}{*}{$\begin{array}{l}\text { Ground- } \\
\text { truth }\end{array}$} & \multicolumn{7}{|c|}{ Demonstrator Classification } \\
\hline & \multirow[b]{2}{*}{ Confidence } & \multicolumn{3}{|c|}{ QA Range } & \multicolumn{3}{|c|}{ Area A } \\
\hline & & $\begin{array}{l}\text { Total } \\
\text { Anomalies }\end{array}$ & $\begin{array}{l}\text { Declared } \\
\text { Ordnance }\end{array}$ & $\begin{array}{l}\text { Declared } \\
\text { Clutter }\end{array}$ & $\begin{array}{l}\text { Total } \\
\text { Anomalies }\end{array}$ & $\begin{array}{l}\text { Declared } \\
\text { Ordnance }\end{array}$ & $\begin{array}{l}\text { Declared } \\
\text { Clutter }\end{array}$ \\
\hline \multirow{4}{*}{$\begin{array}{l}\text { Non- } \\
\text { emplaced }\end{array}$} & High & 104 & 100 & 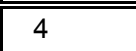 & 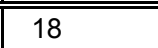 & 18 & 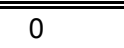 \\
\hline & Low & 291 & 248 & 43 & 53 & 47 & 6 \\
\hline & Medium & \begin{tabular}{|l|}
317 \\
\end{tabular} & 281 & 36 & 48 & 44 & 4 \\
\hline & Total & 712 & 629 & 83 & 119 & 109 & 10 \\
\hline \multirow[t]{4}{*}{ Clutter } & High & 21 & 21 & 0 & 8 & 8 & 0 \\
\hline & Low & 13 & 11 & 2 & 2 & 2 & 0 \\
\hline & Medium & 30 & 28 & 2 & 5 & 5 & 0 \\
\hline & Total & 64 & 60 & 4 & 15 & 15 & 0 \\
\hline \multirow[t]{4}{*}{ Ordnance } & High & 19 & 19 & 0 & 5 & 5 & 0 \\
\hline & Low & 16 & 16 & 0 & 4 & 4 & 0 \\
\hline & Medium & 24 & 24 & 0 & 7 & 7 & 0 \\
\hline & Total & 59 & 59 & 0 & 16 & 16 & 0 \\
\hline Total & & 835 & 748 & 87 & 150 & 140 & 10 \\
\hline
\end{tabular}




\begin{tabular}{|c|c|c|c|c|c|c|c|}
\hline \multicolumn{8}{|c|}{$\begin{array}{l}\text { Table } 20 \\
\text { Breakdown of Discrimination - Area B and Area C - Geophex \& } \\
\text { Geophex without } 20 / 40 \mathrm{~mm}\end{array}$} \\
\hline \multirow[b]{3}{*}{$\begin{array}{l}\text { Ground- } \\
\text { truth }\end{array}$} & \multicolumn{7}{|c|}{ Demonstrator Classification } \\
\hline & \multirow[b]{2}{*}{ Confidence } & \multicolumn{3}{|c|}{ Area B } & \multicolumn{3}{|c|}{ Area C } \\
\hline & & $\begin{array}{l}\text { Total } \\
\text { Anomalies }\end{array}$ & $\begin{array}{l}\text { Declared } \\
\text { Ordnance }\end{array}$ & $\begin{array}{l}\text { Declared } \\
\text { Clutter }\end{array}$ & $\begin{array}{l}\text { Total } \\
\text { Anomalies }\end{array}$ & $\begin{array}{l}\text { Declared } \\
\text { Ordnance }\end{array}$ & $\begin{array}{l}\text { Declared } \\
\text { Clutter }\end{array}$ \\
\hline \multirow{4}{*}{$\begin{array}{l}\text { Non- } \\
\text { emplaced }\end{array}$} & High & 67 & 64 & 3 & 19 & 18 & 1 \\
\hline & Low & 174 & 155 & 19 & 64 & 46 & 18 \\
\hline & Medium & 199 & 174 & 25 & 70 & 63 & 7 \\
\hline & Total & 440 & 393 & 47 & 153 & 127 & 26 \\
\hline \multirow[t]{4}{*}{ Clutter } & High & 10 & 10 & 0 & 3 & 3 & 0 \\
\hline & Low & 8 & 6 & 2 & 3 & 3 & 0 \\
\hline & Medium & 16 & 14 & 2 & 9 & 9 & 0 \\
\hline & Total & 34 & 30 & 4 & 15 & 15 & 0 \\
\hline \multirow[t]{4}{*}{ Ordnance } & High & 9 & 9 & 0 & 5 & 5 & 0 \\
\hline & Low & 10 & 10 & 0 & 2 & 2 & 0 \\
\hline & \begin{tabular}{|l|} 
Medium \\
\end{tabular} & 14 & 14 & 0 & 3 & 3 & 0 \\
\hline & Total & 33 & 33 & 0 & 10 & 10 & 0 \\
\hline Total & & 507 & 456 & 51 & 178 & 152 & 26 \\
\hline
\end{tabular}

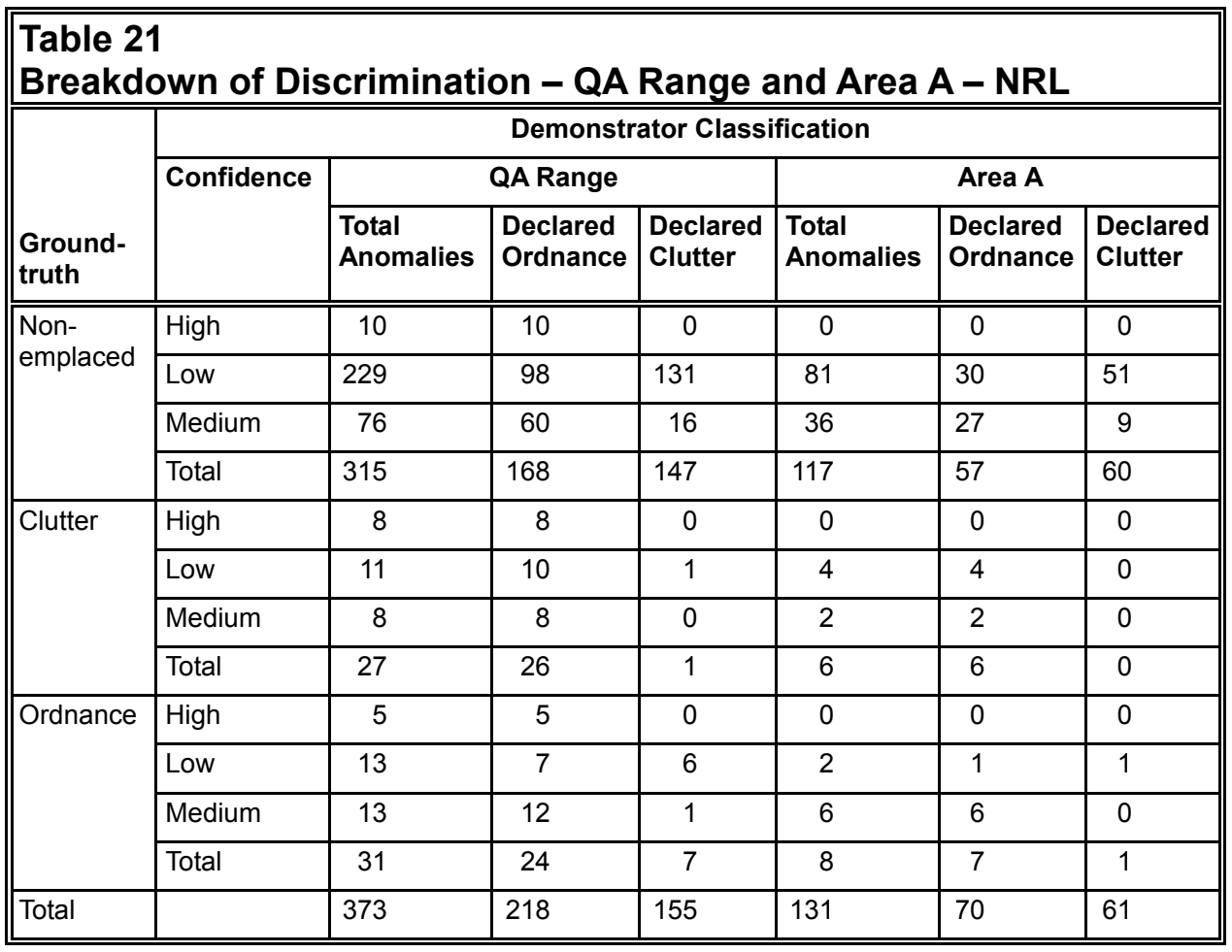




\begin{tabular}{|c|c|c|c|c|c|c|c|}
\hline \multicolumn{8}{|c|}{$\begin{array}{l}\text { Table } 22 \\
\text { Breakdou }\end{array}$} \\
\hline \multirow[b]{3}{*}{$\begin{array}{l}\text { Ground- } \\
\text { truth }\end{array}$} & \multicolumn{7}{|c|}{ Demonstrator Classification } \\
\hline & \multirow[b]{2}{*}{ Confidence } & \multicolumn{3}{|c|}{ Area B } & \multicolumn{3}{|c|}{ Area C } \\
\hline & & \begin{tabular}{|l} 
Total \\
Anomalies
\end{tabular} & $\begin{array}{l}\text { Declared } \\
\text { Ordnance }\end{array}$ & $\begin{array}{l}\text { Declared } \\
\text { Clutter }\end{array}$ & \begin{tabular}{|l} 
Total \\
Anomalies
\end{tabular} & $\begin{array}{l}\text { Declared } \\
\text { Ordnance }\end{array}$ & $\begin{array}{l}\text { Declared } \\
\text { Clutter }\end{array}$ \\
\hline \multirow{4}{*}{$\begin{array}{l}\text { Non- } \\
\text { emplaced }\end{array}$} & High & 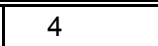 & 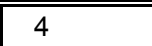 & 0 & 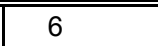 & 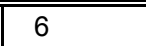 & 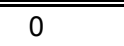 \\
\hline & Low & 83 & 42 & 41 & 65 & 26 & 39 \\
\hline & Medium & 17 & 13 & 4 & 23 & 20 & 3 \\
\hline & Total & 104 & 59 & 45 & 94 & 52 & 42 \\
\hline \multirow[t]{4}{*}{ Clutter } & High & 6 & 6 & 0 & 2 & 2 & 0 \\
\hline & Low & 4 & 3 & 1 & 3 & 3 & 0 \\
\hline & Medium & 4 & 4 & 0 & 2 & 2 & 0 \\
\hline & Total & 14 & 13 & 1 & 7 & 7 & 0 \\
\hline \multirow[t]{4}{*}{ Ordnance } & High & 4 & 4 & 0 & 1 & 1 & 0 \\
\hline & \begin{tabular}{|l} 
Low \\
\end{tabular} & 7 & 4 & 3 & 4 & 2 & 2 \\
\hline & Medium & 6 & 5 & 1 & 1 & 1 & 0 \\
\hline & \begin{tabular}{|l|} 
Total \\
\end{tabular} & 17 & 13 & 4 & 6 & 4 & 2 \\
\hline $\mid$\begin{tabular}{||l|} 
Total \\
\end{tabular} & & 135 & 85 & 50 & 107 & 63 & 44 \\
\hline
\end{tabular}

\begin{tabular}{|c|c|c|c|c|c|c|c|}
\hline \multicolumn{8}{|c|}{$\begin{array}{l}\text { Table } 23 \\
\text { Breakdown of Discrimination - QA Range and Area A- NRL without } \\
20 / 40 \mathrm{~mm}\end{array}$} \\
\hline \multirow[b]{3}{*}{$\begin{array}{l}\text { Ground- } \\
\text { truth }\end{array}$} & \multicolumn{7}{|c|}{ Demonstrator Classification } \\
\hline & \multirow[b]{2}{*}{ Confidence } & \multicolumn{3}{|c|}{ QA Range } & \multicolumn{3}{|c|}{ Area A } \\
\hline & & \begin{tabular}{|l} 
Total \\
Anomalies
\end{tabular} & $\begin{array}{l}\text { Declared } \\
\text { Ordnance }\end{array}$ & $\begin{array}{l}\text { Declared } \\
\text { Clutter }\end{array}$ & \begin{tabular}{|l} 
Total \\
Anomalies
\end{tabular} & $\begin{array}{l}\text { Declared } \\
\text { Ordnance }\end{array}$ & $\begin{array}{l}\text { Declared } \\
\text { Clutter }\end{array}$ \\
\hline \multirow{4}{*}{$\begin{array}{l}\text { Non- } \\
\text { emplaced }\end{array}$} & High & 25 & 25 & 0 & 2 & 2 & 0 \\
\hline & Low & 345 & 163 & 182 & 99 & 46 & 53 \\
\hline & Medium & 197 & 66 & 131 & 62 & 21 & 41 \\
\hline & Total & 567 & 254 & 313 & 163 & 69 & 94 \\
\hline \multirow[t]{4}{*}{ Clutter } & High & 9 & 9 & 0 & 0 & 0 & 0 \\
\hline & Low & 16 & 10 & 6 & 4 & 4 & 0 \\
\hline & Medium & 10 & 10 & 0 & 2 & 2 & 0 \\
\hline & Total & 35 & 29 & 6 & 6 & 6 & 0 \\
\hline \multirow[t]{4}{*}{ Ordnance } & High & 10 & 10 & 0 & 2 & 2 & 0 \\
\hline & Low & 16 & 13 & 3 & 5 & 5 & 0 \\
\hline & Medium & 11 & 7 & 4 & 2 & 1 & 1 \\
\hline & \begin{tabular}{|l|} 
Total \\
\end{tabular} & 37 & 30 & 7 & 9 & 8 & 1 \\
\hline Total & & 639 & 313 & 326 & 178 & 83 & 95 \\
\hline
\end{tabular}




\begin{tabular}{|c|c|c|c|c|c|c|c|}
\hline \multicolumn{8}{|c|}{$\begin{array}{l}\text { Table } 24 \\
\text { Breakdown of Discrimination - Area B and Area C - NRL without } \\
20 / 40 \mathrm{~mm}\end{array}$} \\
\hline \multirow[b]{3}{*}{$\begin{array}{l}\text { Ground- } \\
\text { truth }\end{array}$} & \multicolumn{7}{|c|}{ Demonstrator Classification } \\
\hline & \multirow[b]{2}{*}{ Confidence } & \multicolumn{3}{|c|}{ Area B } & \multicolumn{3}{|c|}{ Area C } \\
\hline & & \begin{tabular}{|l|} 
Total \\
Anomalies
\end{tabular} & $\begin{array}{l}\text { Declared } \\
\text { Ordnance }\end{array}$ & $\begin{array}{l}\text { Declared } \\
\text { Clutter }\end{array}$ & $\begin{array}{l}\text { Total } \\
\text { Anomalies }\end{array}$ & $\begin{array}{l}\text { Declared } \\
\text { Ordnance }\end{array}$ & $\begin{array}{l}\text { Declared } \\
\text { Clutter }\end{array}$ \\
\hline \multirow{4}{*}{$\begin{array}{l}\text { Non- } \\
\text { emplaced }\end{array}$} & High & 5 & 5 & 0 & 18 & 18 & 0 \\
\hline & Low & 163 & 71 & 92 & 83 & 46 & 37 \\
\hline & Medium & 88 & 28 & 60 & 47 & 17 & 30 \\
\hline & Total & 256 & 104 & 152 & 148 & 81 & 67 \\
\hline \multirow[t]{4}{*}{ Clutter } & High & 5 & 5 & 0 & 4 & 4 & 0 \\
\hline & Low & 9 & 5 & 4 & 3 & 1 & 2 \\
\hline & Medium & 5 & 5 & 0 & 3 & 3 & 0 \\
\hline & Total & 19 & 15 & 4 & 10 & 8 & 2 \\
\hline \multirow[t]{4}{*}{ Ordnance } & High & 5 & 5 & 0 & 3 & 3 & 0 \\
\hline & Low & 8 & 6 & 2 & 3 & 2 & 1 \\
\hline & Medium & 7 & 4 & 3 & 2 & 2 & 0 \\
\hline & Total & 20 & 15 & 5 & 8 & 7 & 1 \\
\hline Total & & 295 & 134 & 161 & 166 & 96 & 70 \\
\hline
\end{tabular}

\begin{tabular}{|c|c|c|c|c|c|c|c|}
\hline $\begin{array}{l}\text { Table 2 } \\
\text { Breakd } \\
\text { (EM61) }\end{array}$ & $\begin{array}{l}5 \\
\text { own of Dis } \\
\text { EM and F }\end{array}$ & crimina & $\mathbf{n}-\mathbf{Q A}$ & Range & and Area & A- Parsc & \\
\hline & & & Demonstr & ator Classi & fication & & \\
\hline & & & QA Range & & & Area A & \\
\hline $\begin{array}{l}\text { Ground- } \\
\text { truth }\end{array}$ & Confidence & $\begin{array}{l}\text { Total } \\
\text { Anomalies }\end{array}$ & $\begin{array}{l}\text { Declared } \\
\text { Ordnance }\end{array}$ & $\begin{array}{l}\text { Declared } \\
\text { Clutter }\end{array}$ & \begin{tabular}{|l} 
Total \\
Anomalies
\end{tabular} & $\begin{array}{l}\text { Declared } \\
\text { Ordnance }\end{array}$ & $\begin{array}{l}\text { Declared } \\
\text { Clutter }\end{array}$ \\
\hline Non- & Undeclared & 789 & 783 & 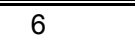 & 123 & 122 & 1 \\
\hline emplaced & High & 8 & 1 & 7 & 1 & 0 & 1 \\
\hline & Low & 1 & 0 & 1 & 1 & 0 & 1 \\
\hline & Medium & 2 & 2 & 0 & 1 & 1 & 0 \\
\hline & Total & 800 & 786 & 14 & 126 & 123 & 3 \\
\hline Clutter & Undeclared & 76 & 76 & 0 & 17 & 17 & 0 \\
\hline & High & 1 & 0 & 1 & 1 & 0 & 1 \\
\hline & Low & 0 & 0 & 0 & 0 & 0 & 0 \\
\hline & Medium & 1 & 1 & 0 & 0 & 0 & 0 \\
\hline & Total & 78 & 77 & 1 & 18 & 17 & 1 \\
\hline Ordnance & Undeclared & 58 & 58 & 0 & 12 & 12 & 0 \\
\hline & High & 0 & 0 & 0 & 0 & 0 & 0 \\
\hline & Low & 1 & 1 & 0 & 0 & 0 & 0 \\
\hline & Medium & 1 & 1 & 0 & 0 & 0 & 0 \\
\hline & Total & 60 & 60 & 0 & 12 & 12 & 0 \\
\hline Total & & 938 & 923 & 15 & 156 & 152 & 4 \\
\hline
\end{tabular}




\begin{tabular}{|c|c|c|c|c|c|c|c|}
\hline \multicolumn{8}{|c|}{$\begin{array}{l}\text { Table } 26 \\
\text { Breakdown of Discrimination - Area B and Area C - Parsons (EM- } \\
\text { 61) EM and FLAG }\end{array}$} \\
\hline \multirow[b]{3}{*}{$\begin{array}{l}\text { Ground- } \\
\text { truth }\end{array}$} & \multicolumn{7}{|c|}{ Demonstrator Classification } \\
\hline & \multirow[b]{2}{*}{ Confidence } & \multicolumn{3}{|c|}{ Area B } & \multicolumn{3}{|c|}{ Area C } \\
\hline & & \begin{tabular}{|l|} 
Total \\
Anomalies \\
\end{tabular} & \begin{tabular}{|l|} 
Declared \\
Ordnance \\
\end{tabular} & \begin{tabular}{|l|} 
Declared \\
Clutter
\end{tabular} & \begin{tabular}{|l|} 
Total \\
Anomalies
\end{tabular} & $\begin{array}{l}\text { Declared } \\
\text { Ordnance }\end{array}$ & $\begin{array}{l}\text { Declared } \\
\text { Clutter }\end{array}$ \\
\hline \multirow{5}{*}{$\begin{array}{l}\text { Non- } \\
\text { emplaced }\end{array}$} & Undeclared & 333 & 328 & 5 & 333 & 333 & 0 \\
\hline & High & 4 & 1 & 3 & 3 & 0 & 3 \\
\hline & Low & 0 & 0 & 0 & 0 & 0 & 0 \\
\hline & Medium & 1 & 1 & 0 & 0 & 0 & 0 \\
\hline & Total & 338 & 330 & 8 & 336 & 333 & 3 \\
\hline \multirow[t]{5}{*}{ Clutter } & Undeclared & 35 & 35 & 0 & 24 & 24 & 0 \\
\hline & High & 0 & 0 & 0 & 0 & 0 & 0 \\
\hline & Low & 0 & 0 & 0 & 0 & 0 & 0 \\
\hline & \begin{tabular}{|l|} 
Medium \\
\end{tabular} & 1 & 1 & 0 & 0 & 0 & 0 \\
\hline & Total & 36 & 36 & 0 & 24 & 24 & 0 \\
\hline \multirow[t]{5}{*}{ Ordnance } & Undeclared & 31 & 31 & 0 & 15 & 15 & 0 \\
\hline & High & 0 & 0 & 0 & 0 & 0 & 0 \\
\hline & Low & 1 & 1 & 0 & 0 & 0 & 0 \\
\hline & \begin{tabular}{|l|} 
Medium \\
\end{tabular} & 1 & 1 & 0 & 0 & 0 & 0 \\
\hline & Total & 33 & 33 & 0 & 15 & 15 & 0 \\
\hline Total & & 407 & 399 & 8 & 375 & 372 & 3 \\
\hline
\end{tabular}

\begin{tabular}{|c|c|c|c|c|c|c|c|}
\hline \multicolumn{8}{|c|}{$\begin{array}{l}\text { Table } 27 \\
\text { Breakdown of Discrimination - QA Range and Area A - Parsons } \\
\text { EM-61 Digital }\end{array}$} \\
\hline \multirow[b]{3}{*}{$\begin{array}{l}\text { Ground- } \\
\text { Truth }\end{array}$} & \multicolumn{7}{|c|}{ Demonstrator Classification } \\
\hline & \multirow[b]{2}{*}{ Confidence } & \multicolumn{3}{|c|}{ QA Range } & \multicolumn{3}{|c|}{ Area A } \\
\hline & & $\begin{array}{l}\text { Total } \\
\text { Anomalies }\end{array}$ & $\begin{array}{l}\text { Declared } \\
\text { Ordnance }\end{array}$ & $\begin{array}{l}\text { Declared } \\
\text { Clutter }\end{array}$ & \begin{tabular}{|l} 
Total \\
Anomalies
\end{tabular} & $\begin{array}{l}\text { Declared } \\
\text { Ordnance }\end{array}$ & $\begin{array}{l}\text { Declared } \\
\text { Clutter }\end{array}$ \\
\hline \multirow{4}{*}{$\begin{array}{l}\text { Non- } \\
\text { emplaced }\end{array}$} & High & 249 & 96 & 153 & 61 & 18 & 43 \\
\hline & Low & 109 & 0 & 109 & 62 & 0 & 62 \\
\hline & Medium & 1,149 & 1 & 1,148 & 258 & 1 & 257 \\
\hline & Total & 1,507 & 97 & 1,410 & 381 & 19 & 362 \\
\hline \multirow[t]{4}{*}{ Clutter } & High & 33 & 23 & 10 & 13 & 10 & 3 \\
\hline & Low & 1 & 0 & 1 & 1 & 0 & 1 \\
\hline & Medium & 27 & 0 & 27 & 3 & 0 & 3 \\
\hline & Total & 61 & 23 & 38 & 17 & 10 & 7 \\
\hline \multirow[t]{4}{*}{ Ordnance } & High & 37 & 26 & 11 & 8 & 5 & 3 \\
\hline & Low & 1 & 0 & 1 & 1 & 0 & 1 \\
\hline & Medium & 14 & 0 & 14 & 3 & 0 & 3 \\
\hline & Total & 52 & 26 & 26 & 12 & 5 & 7 \\
\hline Total & & 1,620 & 146 & 1,474 & 410 & 34 & 376 \\
\hline
\end{tabular}




\begin{tabular}{|c|c|c|c|c|c|c|c|}
\hline \multicolumn{8}{|c|}{$\begin{array}{l}\text { Table } 28 \\
\text { Breakdown of Discrimination - Area B and Area C - Parsons EM-61 } \\
\text { Digital }\end{array}$} \\
\hline \multirow[b]{3}{*}{$\begin{array}{l}\text { Ground- } \\
\text { truth }\end{array}$} & \multicolumn{7}{|c|}{ Demonstrator Classification } \\
\hline & \multirow[b]{2}{*}{ Confidence } & \multicolumn{3}{|c|}{ Area B } & \multicolumn{3}{|c|}{ Area C } \\
\hline & & \begin{tabular}{|l|} 
Total \\
Anomalies
\end{tabular} & \begin{tabular}{|l|} 
Declared \\
Ordnance
\end{tabular} & \begin{tabular}{|l|} 
Declared \\
Clutter
\end{tabular} & \begin{tabular}{|l|} 
Total \\
Anomalies
\end{tabular} & \begin{tabular}{|l|} 
Declared \\
Ordnance
\end{tabular} & $\begin{array}{l}\text { Declared } \\
\text { Clutter }\end{array}$ \\
\hline \multirow{4}{*}{$\begin{array}{l}\text { Non- } \\
\text { emplaced }\end{array}$} & High & 81 & 44 & 37 & 107 & 34 & 73 \\
\hline & Low & 3 & 0 & 3 & 44 & 0 & 44 \\
\hline & Medium & 458 & 0 & 458 & 433 & 0 & 433 \\
\hline & Total & 542 & 44 & 498 & 584 & 34 & 550 \\
\hline \multirow[t]{4}{*}{ Clutter } & High & 9 & 3 & 6 & 11 & 10 & 1 \\
\hline & Low & 0 & 0 & 0 & 0 & 0 & 0 \\
\hline & Medium & 17 & 0 & 17 & 7 & 0 & 7 \\
\hline & Total & 26 & 3 & 23 & 18 & 10 & 8 \\
\hline \multirow[t]{4}{*}{ Ordnance } & High & 18 & 13 & 5 & 11 & 8 & 3 \\
\hline & Low & 0 & 0 & 0 & 0 & 0 & 0 \\
\hline & Medium & 7 & 0 & 7 & 4 & 0 & 4 \\
\hline & \begin{tabular}{|l|} 
Total \\
\end{tabular} & 25 & 13 & 12 & 15 & 8 & 7 \\
\hline Total & & 593 & 60 & 533 & 617 & 52 & 565 \\
\hline
\end{tabular}

\begin{tabular}{|c|c|c|c|c|c|c|c|}
\hline \multicolumn{8}{|c|}{$\begin{array}{l}\text { Table } 29 \\
\text { Breakdown of Discrimination - QA Range and Area A - Parsons } \\
\text { TM-5 EMU }\end{array}$} \\
\hline \multirow[b]{3}{*}{$\begin{array}{l}\text { Ground } \\
\text { Truth }\end{array}$} & \multicolumn{7}{|c|}{ Demonstrator Classification } \\
\hline & \multirow[b]{2}{*}{ Confidence } & \multicolumn{3}{|c|}{ QA Range } & \multicolumn{3}{|c|}{ Area A } \\
\hline & & \begin{tabular}{|l} 
Total \\
Anomalies
\end{tabular} & $\begin{array}{l}\text { Declared } \\
\text { Ordnance }\end{array}$ & $\begin{array}{l}\text { Declared } \\
\text { Clutter }\end{array}$ & $\begin{array}{l}\text { Total } \\
\text { Anomalies }\end{array}$ & $\begin{array}{l}\text { Declared } \\
\text { Ordnance }\end{array}$ & $\begin{array}{l}\text { Declared } \\
\text { Clutter }\end{array}$ \\
\hline \multirow{3}{*}{$\begin{array}{l}\text { Non- } \\
\text { emplaced }\end{array}$} & Undeclared & 135 & 131 & 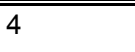 & 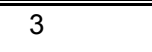 & 3 & 0 \\
\hline & High & 3 & 1 & 2 & 0 & 0 & 0 \\
\hline & Total & 138 & 132 & 6 & 3 & 3 & 0 \\
\hline \multirow[t]{3}{*}{ Clutter } & Undeclared & 36 & 36 & 0 & 8 & 8 & 0 \\
\hline & High & 0 & 0 & 0 & 0 & 0 & 0 \\
\hline & Total & 36 & 36 & 0 & 8 & 8 & 0 \\
\hline \multirow[t]{3}{*}{ Ordnance } & Undeclared & 20 & 20 & 0 & 6 & 6 & 0 \\
\hline & High & 0 & 0 & 0 & 0 & 0 & 0 \\
\hline & Total & 20 & 20 & 0 & 6 & 6 & 0 \\
\hline Total & & 194 & 188 & 6 & 17 & 17 & 0 \\
\hline
\end{tabular}




\begin{tabular}{|c|c|c|c|c|c|c|c|}
\hline \multicolumn{8}{|c|}{$\begin{array}{l}\text { Table } 30 \\
\text { Breakdown of Discrimination - Area B and Area C - Parsons TM-5 } \\
\text { EMU }\end{array}$} \\
\hline \multirow[b]{3}{*}{$\begin{array}{l}\text { Ground- } \\
\text { truth }\end{array}$} & \multicolumn{7}{|c|}{ Demonstrator Classification } \\
\hline & \multirow[b]{2}{*}{ Confidence } & \multicolumn{3}{|c|}{ Area B } & \multicolumn{3}{|l|}{ Area C } \\
\hline & & $\begin{array}{l}\text { Total } \\
\text { Anomalies }\end{array}$ & $\begin{array}{l}\text { Declared } \\
\text { Ordnance }\end{array}$ & $\begin{array}{l}\text { Declared } \\
\text { Clutter }\end{array}$ & $\begin{array}{l}\text { Total } \\
\text { Anomalies }\end{array}$ & $\begin{array}{l}\text { Declared } \\
\text { Ordnance }\end{array}$ & $\begin{array}{l}\text { Declared } \\
\text { Clutter }\end{array}$ \\
\hline \multirow{3}{*}{$\begin{array}{l}\text { Non- } \\
\text { emplaced }\end{array}$} & Undeclared & 112 & 109 & 3 & 20 & 19 & $\overline{1}$ \\
\hline & High & 3 & 1 & 2 & 0 & 0 & 0 \\
\hline & Total & 115 & 110 & 5 & 20 & 19 & 1 \\
\hline \multirow[t]{3}{*}{ Clutter } & Undeclared & 25 & 25 & 0 & 3 & 3 & 0 \\
\hline & High & 0 & 0 & 0 & 0 & 0 & 0 \\
\hline & Total & 25 & 25 & 0 & 3 & 3 & 0 \\
\hline \multirow[t]{3}{*}{ Ordnance } & Undeclared & 13 & 13 & 0 & 1 & 1 & 0 \\
\hline & High & 0 & 0 & 0 & 0 & 0 & 0 \\
\hline & Total & 13 & 13 & 0 & 1 & 1 & 0 \\
\hline Total & & 153 & 148 & 5 & 24 & 23 & 1 \\
\hline
\end{tabular}

\section{Identification Results}

The UXO identification performance of each demonstrator is based on the UXO type declarations included in each of the required prioritized dig lists. The results presented in this section have been adjusted to account for UXO-related items that were present in Areas A, B, and C from previous demonstration work. Figures 32 through 36 include classification matrices that detail and summarize the identification performance of each demonstrator. There are two types of classification matrices presented.

The first type of classification matrix is the target classification matrix, which shows how well the demonstrator identified specific ordnance types that were detected. This classification matrix includes the following entries:

a. The entry below the demonstrator's name indicates the demonstration Area. Area A is the four 30-m grid, Area B is the 1-hectare site, and Area $\mathrm{C}$ is the six $30-\mathrm{m}$ grid.

$b$. The classes across the top of the matrix are the classification types of the actual target classes. A list of these types was given to the demonstrators for use in classifying their detected ordnance. A clutter class is listed as a separate entry and corresponds to a false alarm if classified as a UXO target and to a correct discrimination when classified as non-UXO by the demonstrator.

c. The classification column on the left side of the matrix lists the demonstrator's declaration for each detected UXO item.

d. The totals in the right-hand column of the matrix correspond to the total number of items declared by the demonstrator as a particular class. 
e. The "\% Classified" row indicates the percentage of detected targets, for a given target class, that were correctly classified.

$f$. The "\% Classified by Class" indicates the percentage of detected targets, for the given classes of projectile, mortar, rocket, and practice bomb, that were correctly classified.

g. The "\% of Total Detected Targets Classified as Nonordnance Low/Med Confidence" is the percentage of detected ordnance that the demonstrator incorrectly classified as nonordnance with low or medium confidence.

h. The "\% of Total Detected Targets Classified as Nonordnance High Confidence" is the percentage of detected ordnance that the demonstrator incorrectly classified as nonordnance with high confidence. This classification error carried the highest cost penalty (equivalent to the cost of a complete resurvey of the area).

Note that only one demonstrator classified their declarations with the given target classes.

The second type of classification matrix is the aggregate size classification matrix. This matrix includes the following entries:

a. The entry below the demonstrator's name indicates the demonstration Area.

$b$. The classes across the top of the matrix are the aggregate size classes of the items emplaced. The targets are grouped into three classes. The small target class contains the $20-\mathrm{mm}$ projectile, the $40-\mathrm{mm}$ projectile, and the BDU 3 practice bomb. The medium class contains the $60-\mathrm{mm}$ mortar, the 81-mm mortar, the 2.25-in. rocket, the 2.75-in. rocket warhead, the SMAW rocket, the LAAW, the BDU 33 practice bomb, the MK-3 practice bomb, and the MK-106 practice bomb. The large class contains the 5-in. projectile; the $105-\mathrm{mm}$ projectile, the MK- 81 practice bomb, the MK- 82 practice bomb, and the MK- 83 practice bomb. A clutter class is listed as a separate entry and corresponds to a false alarm if classified as a UXO target and to a correct discrimination when classified as non$\mathrm{UXO}$ by the demonstrator.

c. The classification column on the left side of the matrix lists the demonstrator's declaration for each detected UXO item. Again, the declarations are grouped into three aggregate size classes as previously described in the target classes.

d. The totals in the right-hand column of the matrix correspond to the total number of items declared by the demonstrator as a particular class.

e. The "\% Classified" row indicates the percentage of detected targets, for a given aggregate size class, that were correctly classified.

f. The "\% of Total Detected Targets Classified as Nonordnance Low/Med Confidence" is the percentage of detected ordnance that the demonstrator incorrectly classified as nonordnance with low or medium confidence. 
g. The "\% of Total Detected Targets Classified as Nonordnance High Confidence" is the percentage of detected ordnance that the demonstrator incorrectly classified as nonordnance with high confidence. Again, this classification error carried the highest cost penalty (equivalent to the cost of a complete resurvey of the area).

Note that only two demonstrators classified their declarations with enough descriptive information to allow for the evaluation of aggregate size classification.

NAEVA was the only demonstrator that submitted specific target identifications. However, their classification results, shown in Figures 32 through 34, were very poor. Their results are only slightly better when aggregated by size, as shown in Figures 35. Classification matrices aggregated by size for Geophex are shown in Figures 36. Geophex correctly classified a few more ordnance by size than NAEVA, but also classified more nonordnance items as ordnance. NAEVA and Geophex are the only demonstrators who attempted to identify ordnance by size.

\section{ROC-Based Performance Assessment}

\section{Assessment of detection, discrimination, and false alarm rate performance}

One of the critical evaluation factors for this demonstration is the detection and discrimination performance of the advanced systems as a function of the number of false alarms. The metrics used to quantify this performance consist of the pseudo ROC curves, the single-point $\mathrm{P}(\mathrm{det}) /$ Total FAR, and the maximum achievable $\mathrm{P}(\mathrm{det})$. The methods used to estimate these metrics from the prioritized dig lists are described in detail in Chapter 4. Briefly, the pseudo ROC curve, which graphically represents the target detection percentage vs. the total number of false alarms (or false alarm rate in number of false alarms per hectare), is calculated by sequentially moving from the top of the prioritized dig list (i.e., the highest confidence UXO target declaration) and determining if each object on the list (whether classified as target or clutter) corresponds to an emplaced target location (a correct detection) or not (a false alarm). The singlepoint $\mathrm{P}(\mathrm{det}) /$ Total FAR performance is based on the point on the ROC curve that corresponds to the demonstrator-specified dig point on the prioritized dig list, and the maximum achievable $\mathrm{P}(\mathrm{det})$ is based on the highest point on the ROC curve. These performance metrics are presented in the following graphs. The singlepoint $\mathrm{P}(\mathrm{det}) /$ Total FAR rate is shown as a triangle on the ROC curve (stop-digpoint) and it is noted by "SD" in the legend. The probability of correct discrimination, $\mathrm{P}(\operatorname{disc})$, was plotted on the signal strength ROC curves.

\section{Interpreting pseudo-ROC curves}

There are several points to keep in mind when interpreting these pseudo ROC curves: 


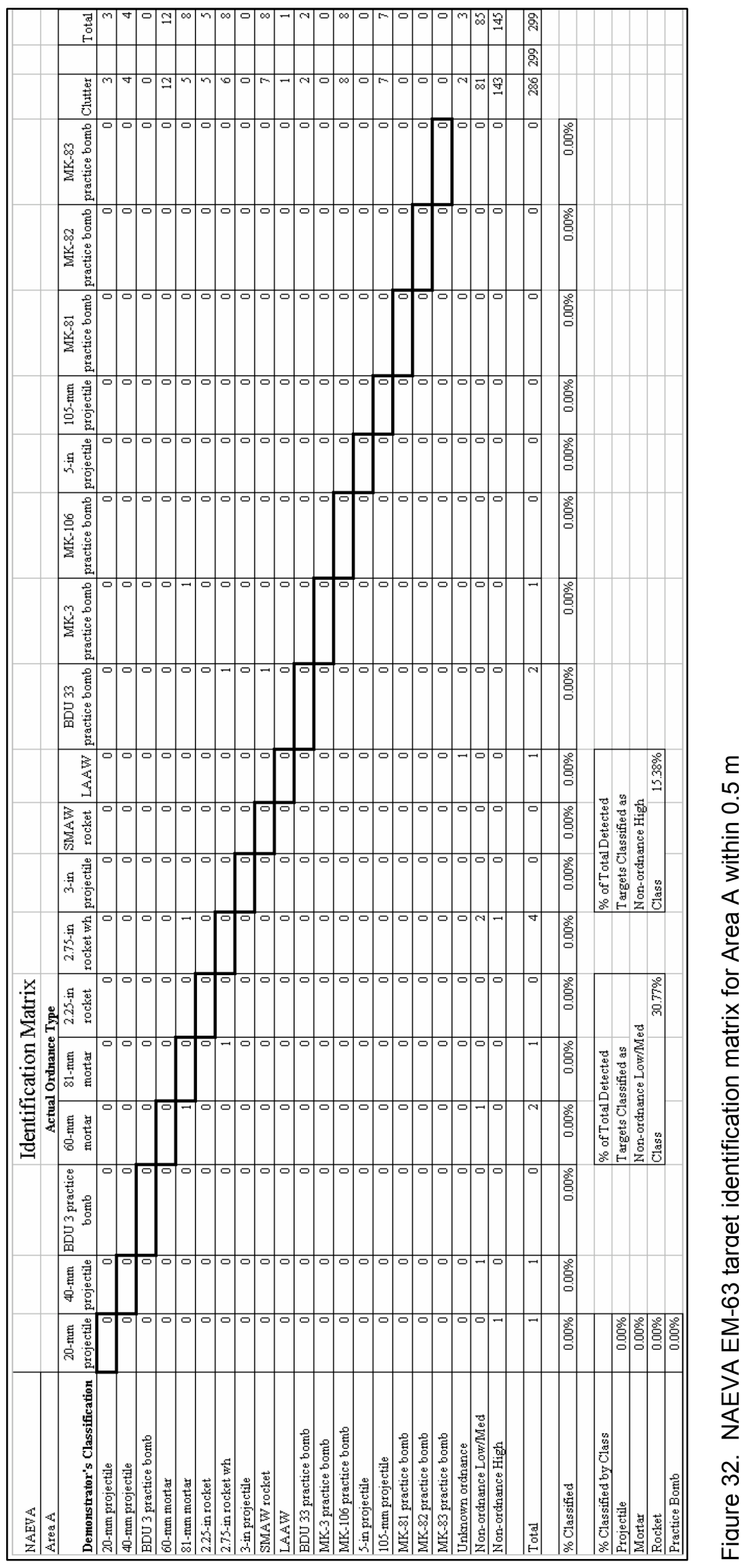




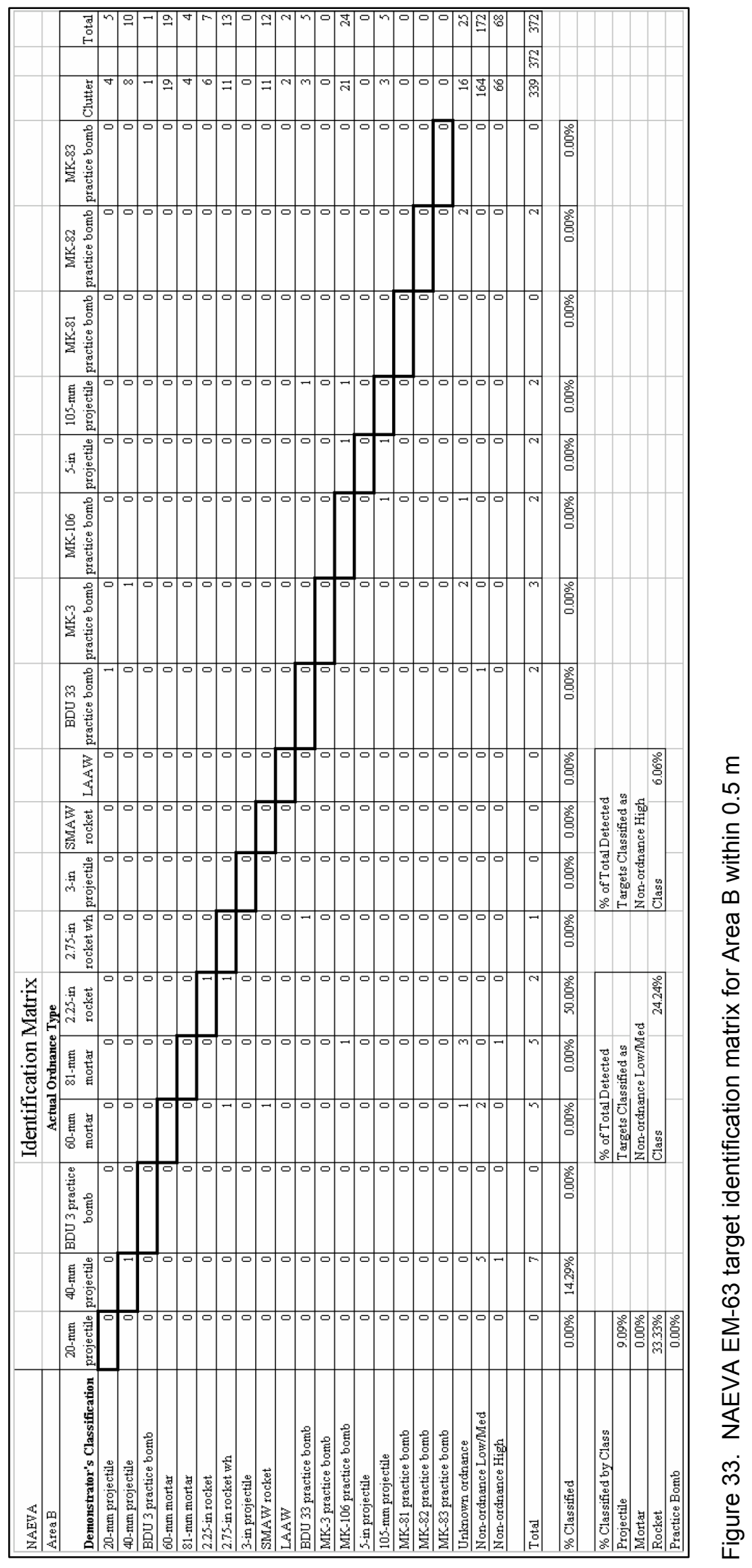




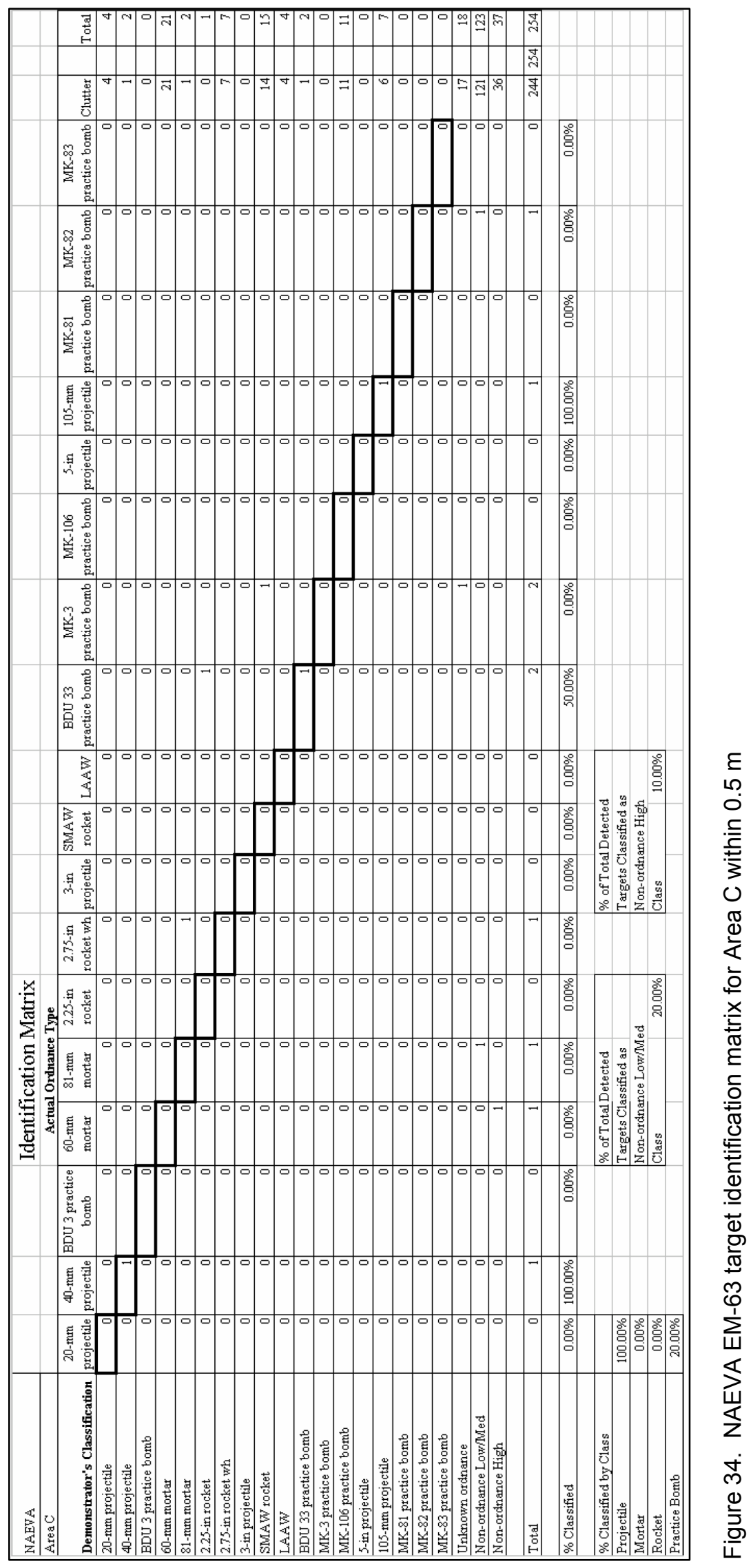




\begin{tabular}{|c|c|c|c|c|c|c|}
\hline \multirow{3}{*}{\begin{tabular}{|l} 
NAEVA \\
Area A \\
Demonstrator's Classification \\
\end{tabular}} & \multirow{2}{*}{\multicolumn{5}{|c|}{$\begin{array}{c}\text { Classification Matrix } \\
\text { Target Classification by Size }\end{array}$}} & \\
\hline & & & & & & \\
\hline & small & medium & large & Clutter & & Total \\
\hline small & 0 & 0 & 0 & 7 & & 7 \\
\hline medium & 0 & 6 & 0 & 46 & & 52 \\
\hline large & 0 & 0 & 0 & 7 & & 7 \\
\hline Unknown ordnance & 0 & 1 & 0 & 2 & & 3 \\
\hline Non-ordnance Low/Med & 1 & 3 & 0 & 81 & & 85 \\
\hline \begin{tabular}{|l|} 
Non-ordnance High \\
\end{tabular} & 1 & 1 & 0 & 143 & & 145 \\
\hline Total & 2 & 11 & 0 & 286 & 299 & 299 \\
\hline \multirow{3}{*}{$\%$ Classified } & $0.00 \%$ & $54.55 \%$ & $0.00 \%$ & & & \\
\hline & & \multicolumn{2}{|c|}{$\begin{array}{l}\% \text { of Total Detected } \\
\text { Targets Classified as } \\
\text { Non-ordnance Low/Med }\end{array}$} & & \multicolumn{2}{|c|}{$\begin{array}{l}\text { \% of Total Detected } \\
\text { Targets Classified as } \\
\text { Non-ordnance High }\end{array}$} \\
\hline & & Class & $30.77 \%$ & & Class & $15.38 \%$ \\
\hline
\end{tabular}

\begin{tabular}{|c|c|c|c|c|c|c|}
\hline \multirow{2}{*}{\begin{tabular}{|l|} 
NAEVA \\
Area B \\
\end{tabular}} & \multirow{2}{*}{\multicolumn{3}{|c|}{$\begin{array}{c}\text { Classification Matrix } \\
\text { Target Classification by Size }\end{array}$}} & & & \\
\hline & & & & & & \\
\hline Demonstrator's Classification & small & medium & large & Clutter & & Total \\
\hline small & 1 & 2 & 0 & 13 & & 16 \\
\hline medium & 0 & 6 & 3 & 77 & & 86 \\
\hline large & 0 & 1 & 1 & 3 & & 5 \\
\hline Unknown ordnance & 0 & 7 & 2 & 16 & & 25 \\
\hline Non-ordnance Low/Med & 5 & 3 & 0 & 164 & & 172 \\
\hline Non-ordnance High & 1 & 1 & 0 & 66 & & 68 \\
\hline Total & 7 & 20 & 6 & 339 & 372 & 37 \\
\hline & & & & & & \\
\hline$\%$ Classified & $14.29 \%$ & $30.00 \%$ & $16.67 \%$ & & & \\
\hline & & $\begin{array}{l}\text { \% of Total Detected } \\
\text { Targets Classified as } \\
\text { Non-ordnance Low/l }\end{array}$ & Med & & $\begin{array}{l}\% \text { of } \\
\text { Targe } \\
\text { Non-c }\end{array}$ & $\begin{array}{l}\text { Total Detected } \\
\text { ts Classified as } \\
\text { ordnance High }\end{array}$ \\
\hline & & Class & $24.24 \%$ & & Class & $6.06 \%$ \\
\hline
\end{tabular}

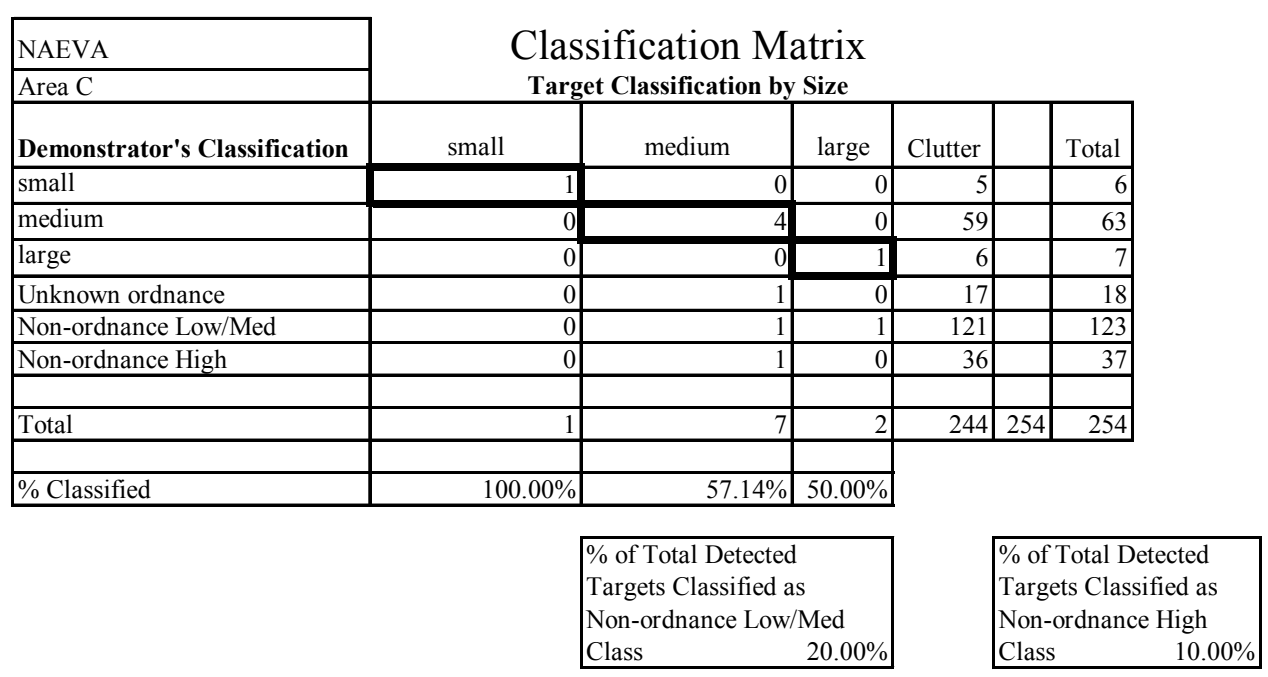

Figure 35. NAEVA EM-63 size classification matrix within $0.5 \mathrm{~m}$ 


\begin{tabular}{|c|c|c|c|c|c|c|c|}
\hline \multirow{3}{*}{\begin{tabular}{|l|} 
Geophex \\
Area A \\
Demonstrator's Classification
\end{tabular}} & \multirow{2}{*}{\multicolumn{6}{|c|}{$\begin{array}{c}\text { Classification Matrix } \\
\text { Target Classification by Size }\end{array}$}} & \\
\hline & & & & & & & \\
\hline & small & medium & large & Clutter & & Total & \\
\hline small & 2 & 1 & 0 & 23 & & 26 & \\
\hline medium & 0 & 7 & 2 & 55 & & 64 & \\
\hline large & 1 & 2 & 1 & 46 & & 50 & \\
\hline Unknown ordnance & 0 & 0 & 0 & 0 & & 0 & \\
\hline Non-ordnance Low/Med & 0 & 0 & 0 & 10 & & 10 & \\
\hline Non-ordnance High & 0 & 0 & 0 & 0 & & 0 & \\
\hline Total & 3 & 10 & 3 & 134 & 150 & 150 & \\
\hline$\%$ Classified & $66.67 \%$ & $70.00 \%$ & $33.33 \%$ & & & & \\
\hline & & $\begin{array}{l}\% \text { of Total Detected } \\
\text { Targets Classified a } \\
\text { Non-ordnance Low/ }\end{array}$ & Med & & $\begin{array}{l}\% \text { of } \\
\text { Targ } \\
\text { Non- }\end{array}$ & $\begin{array}{l}\text { Total D } \\
\text { ets Class } \\
\text { ordnanc }\end{array}$ & $\begin{array}{l}\text { eted } \\
\text { ed as } \\
\text { ligh }\end{array}$ \\
\hline & & Class & $0.00 \%$ & & Class & & $0.00 \%$ \\
\hline
\end{tabular}

\begin{tabular}{|c|c|c|c|c|c|c|c|}
\hline \multirow{3}{*}{\begin{tabular}{|l} 
Geophex \\
Area B \\
Demonstrator's Classification
\end{tabular}} & \multirow{2}{*}{\multicolumn{6}{|c|}{$\begin{array}{c}\text { Classification Matrix } \\
\text { Target Classification by Size }\end{array}$}} & \\
\hline & & & & & & & \\
\hline & small & medium & large & Clutter & & Total & \\
\hline small & 2 & 1 & 0 & 64 & & 67 & \\
\hline medium & 3 & 9 & 1 & 202 & & 215 & \\
\hline large & 0 & 10 & 7 & 157 & & 174 & \\
\hline Unknown ordnance & 0 & 0 & 0 & 0 & & 0 & \\
\hline Non-ordnance Low/Med & 0 & 0 & 0 & 51 & & 51 & \\
\hline Non-ordnance High & 0 & 0 & 0 & 0 & & 0 & \\
\hline Total & 5 & 20 & 8 & 474 & 507 & 507 & \\
\hline$\%$ Classified & $40.00 \%$ & $45.00 \%$ & $87.50 \%$ & & & & \\
\hline & & $\begin{array}{l}\% \text { of Total Detected } \\
\text { Targets Classified a } \\
\text { Non-ordnance Low/ } \\
\text { Class }\end{array}$ & Med & & $\begin{array}{l}\% \text { of } \\
\text { Targ } \\
\text { Non } \\
\text { Clas }\end{array}$ & $\begin{array}{l}\text { Total D } \\
\text { ets Clas } \\
\text { ordnan }\end{array}$ & $\begin{array}{l}\text { cted } \\
\text { ed as } \\
\text { ligh }\end{array}$ \\
\hline
\end{tabular}

\begin{tabular}{|c|c|c|c|c|c|c|c|}
\hline \multirow{3}{*}{$\begin{array}{l}\text { Geophex } \\
\text { Area C } \\
\text { Demonstrator's Classification }\end{array}$} & \multirow{2}{*}{\multicolumn{6}{|c|}{$\begin{array}{c}\text { Classification Matrix } \\
\text { Target Classification by Size }\end{array}$}} & \\
\hline & & & & & & & \\
\hline & small & medium & large & Clutter & & Total & \\
\hline small & 1 & 2 & 0 & 25 & & 28 & \\
\hline medium & 0 & 1 & 0 & 61 & & 62 & \\
\hline large & 0 & 5 & 1 & 56 & & 62 & \\
\hline Unknown ordnance & 0 & 0 & 0 & 0 & & 0 & \\
\hline Non-ordnance Low/Med & 0 & 0 & 0 & 26 & & 26 & \\
\hline Non-ordnance High & 0 & 0 & 0 & 0 & & 0 & \\
\hline Total & 1 & 8 & 1 & 168 & 178 & 178 & \\
\hline$\%$ Classified & $100.00 \%$ & $12.50 \%$ & $100.00 \%$ & & & & \\
\hline & & $\begin{array}{l}\% \text { of Total Detected } \\
\text { Targets Classified as } \\
\text { Non-ordnance Low/1 }\end{array}$ & Med & & $\begin{array}{l}\% \text { of } \\
\text { Targe } \\
\text { Non-c }\end{array}$ & $\begin{array}{l}\text { Total D } \\
\text { ets Clas } \\
\text { ordnanc }\end{array}$ & $\begin{array}{l}\text { cted } \\
\text { ed as } \\
\text { ligh }\end{array}$ \\
\hline & & Class & $0.00 \%$ & & Class & & $0.00 \%$ \\
\hline
\end{tabular}

Figure 36. Geophex, Ltd. GEM-3 size classification matrix within $0.5 \mathrm{~m}$ 
a. Abscissa in the pseudo ROC curves is not probability of false alarms but rather total number of false alarms in a hectare or, equivalently, Total FAR (number of false alarms per hectare). Therefore, since each of the Demonstration Areas A, B, and C are different sizes, the Total FARs are adjusted to the ratio of false alarms per hectare so that rate comparisons across different demonstration areas can be made.

$b$. These curves combine detection and discrimination of ordnance from nonordnance. Thus, the initial pseudo ROC curve's slope represents the anomalies that the demonstrator has declared as UXO with the highest confidence; a flat slope in this area would indicate very poor discrimination capability.

c. Similarly, the final slope of the pseudo curve represents anomalies that the demonstrator has declared as clutter with high confidence; a positive slope in this area indicates that there are UXO targets which the demonstrator would leave unexcavated.

Figures 37 through 51 show the results obtained when the location accuracy is set to $0.5 \mathrm{~m}$. In Appendix C (Figures C5 through C20), the analysis is performed with the location accuracy set to $1.0 \mathrm{~m}$. The ROC-based performance of all of the demonstrators in all cases fell below the Kaho'olawe Tier II Clearance requirements. In Figure 37, Geophex demonstrated significantly better ROC performance than the other demonstrators, and GTL demonstrated significantly poorer performance (see Figures 39, 45, 48, and 51).

Figures 37, 38, and 39 show the demonstrators' detection performance for Areas A, B, and C, respectively. The distance threshold for scoring a detection is set to $0.5 \mathrm{~m}$ for these sets of plots. Figure 37 shows that in Area A, most of the systems operated along very similar ROC curves, with the major difference being their selection of the "stop dig" or end point threshold. In the cases of NRL and Parsons TM-5 EMU, the endpoint thresholds were set so high that the operating (and maximum achievable) P(det) was much lower than those of the other demonstrators. Geophex achieved the best performance for this area; furthermore, it is apparent from the steep slope of the ROC curve, that a lower endpoint threshold would probably have resulted in increased $\mathrm{P}(\mathrm{det})$ with a relatively small increase in total FAR.

The placement of the "stop dig" point, (shown as the "SD" triangle in each of these figures) indicates the demonstrators' general lack of confidence in their discrimination capability. With the exception of NAEVA, all other demonstrators placed their "stop dig" point at the end of the dig list. NAEVA's attempt at discrimination resulted in an operating $\mathrm{P}(\mathrm{det})$ that was 10 percent lower than the maximum achievable in Area A.

Figure 38 shows that all demonstrators, with the exception of GTL, achieved very similar ROC-based performance at Area B. GTL demonstrated significantly poorer detection and total FAR performance than the others. Overall, all of the systems demonstrated poorer ROC-based performance in Area B than Area A, confirming previous reports that Area B contained significantly higher levels of 


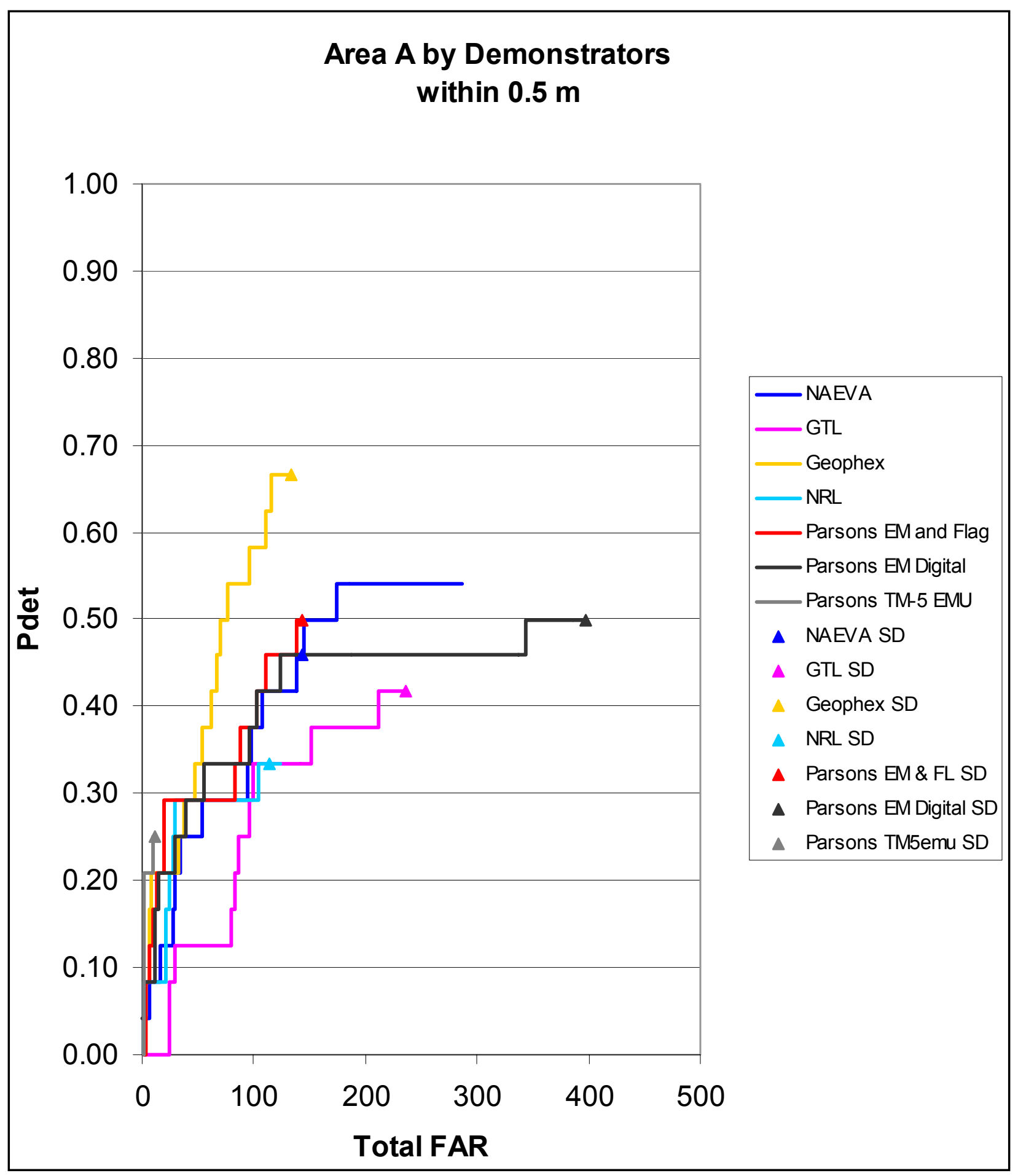

Figure 37. Area $A-P($ det $)$ vs. total $F A R$ within $0.5 \mathrm{~m}$ for all demonstrators 


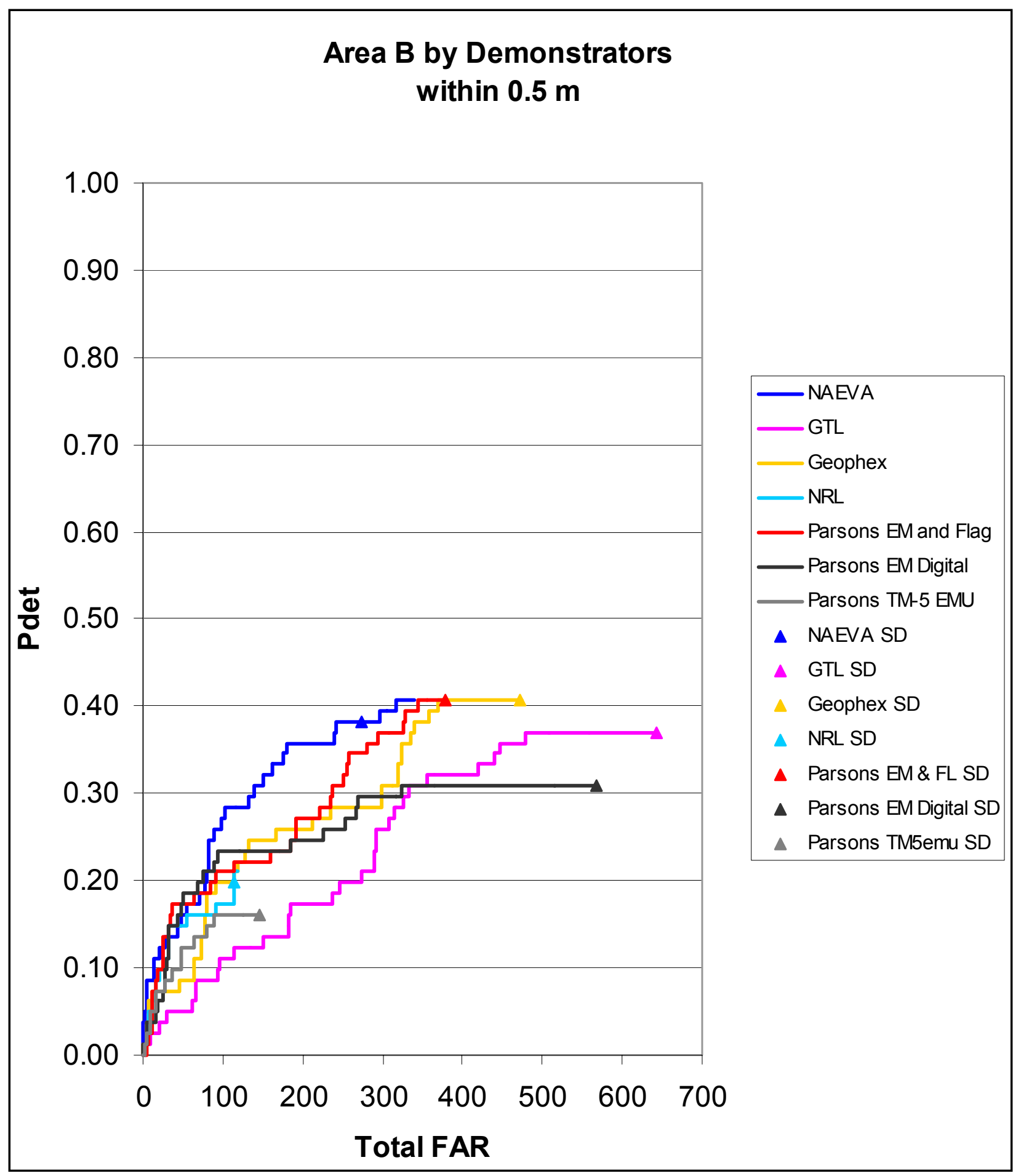

Figure 38. Area $B-P($ det $)$ vs. total $F A R$ within $0.5 \mathrm{~m}$ for all demonstrators 


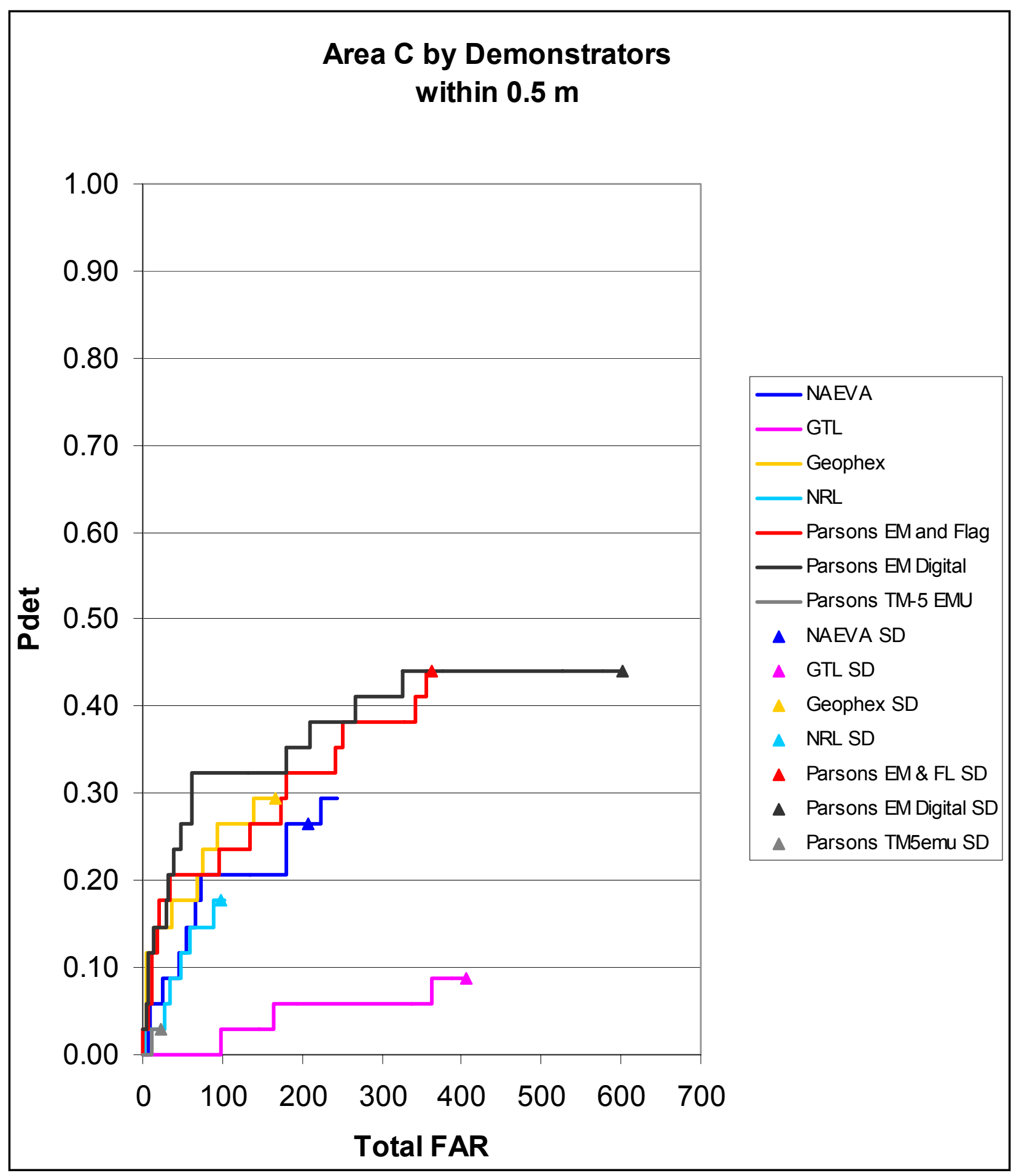

Figure 39. Area $\mathrm{C}-\mathrm{P}(\mathrm{det}) \mathrm{vs}$. total $\mathrm{FAR}$ within $0.5 \mathrm{~m}$ for all demonstrators 
geologic anomalies and metallic clutter. As in Area A, all demonstrators with the exception of NAEVA, were extremely conservative in their "stop dig" point selection.

Figure 39 shows that all of the demonstrators operated along very similar ROC curves within Area C. Again, all of the threshold selections were very conservative. With the exception of Geophex and NRL, all other demonstrators performed better in area $\mathrm{C}$ than $\mathrm{B}$, but none performed better than in Area A.

Figures 40 through 42 show the results of reanalyzing the data to include only targets larger than $40 \mathrm{~mm}$ projectiles. Only NRL and Geophex performed this analysis and resubmitted revised dig lists.

The small number of submissions and the lack of such data from Parsons precludes any comparisons across systems or with a baseline. In order to overcome this deficiency, the demonstrators dig lists that contained all targets (including 20 and $40 \mathrm{~mm}$ ) were evaluated with the $20 / 40 \mathrm{~mm}$ targets removed from the groundtruth. No detection, false alarm, or missed target was assigned to any declaration within $1.0 \mathrm{~m}$ of these small emplaced targets. Figures 43 through 45 show the results of this evaluation. These ROC curves show a slight improvement in performance for all demonstrators, but no significant change was shown in ROC curve shape or in the relative performance between demonstrators. It can be concluded that all systems have similar capability (or lack thereof) to detect the smaller targets in the Kaho'olawe environment.

Figures 46 through 48 show the $\mathrm{P}(\mathrm{det})$ performance as a function of Pfp for all demonstrators, where Pfp is computed as the ratio of the number of emplaced clutter items included on the dig list to the number of clutter items emplaced. This metric attempted to separate the effects of the geology and unknown metal clutter from those from known, emplaced clutter items. These ROC curves show very small differences between demonstrators, but most importantly, the almost consistently flat, diagonal shape of the curves indicates that none of the systems demonstrated a capability to discriminate emplaced UXO from emplaced metallic clutter in this environment.

Figures 49 through 51 show the systems' ability to discriminate ordnance from nonordnance (both geologic and metallic) based solely on signal strength. The ROC curves for all demonstrators tend to fall within a narrow band, and do not support any conclusions regarding the various systems' discrimination capability.

\section{Technology Comparison}

The detection performance of all demonstrated systems was considerably lower than expected and significantly lower than those demonstrated during prior field demonstrations such as JPG Phases II through IV and during the first phase of this project. None of the systems demonstrated ability to discriminate ordnance from metallic clutter nor to identify ordnance by size or type. It was not 


\section{Area A by Demonstrators wo $20 / 40 \mathrm{~mm}$ within $0.5 \mathrm{~m}$}

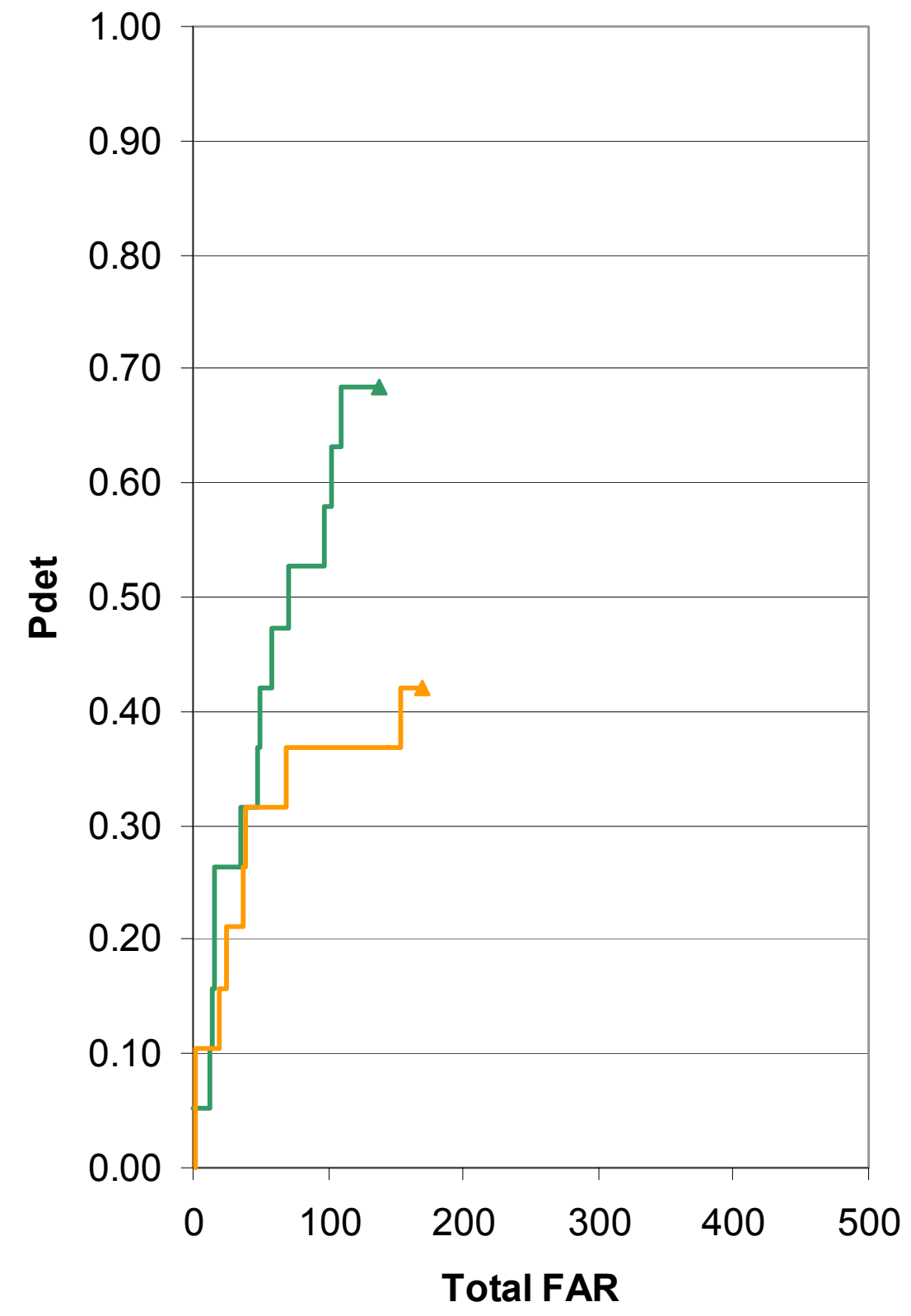

Geophex w o 20/40 mm NRL w o $20 / 40 \mathrm{~mm}$

- Geophex w o 20/40 mm SD

$\triangle \quad$ NRL w o $20 / 40 \mathrm{~mm} \mathrm{SD}$

Figure 40. Area $A-P($ det) vs. total FAR within $0.5 \mathrm{~m}$ for demonstrators without (wo) $20 / 40 \mathrm{~mm}$ 


\section{Area B by Demonstrators wo $20 / 40 \mathrm{~mm}$ within $0.5 \mathrm{~m}$}

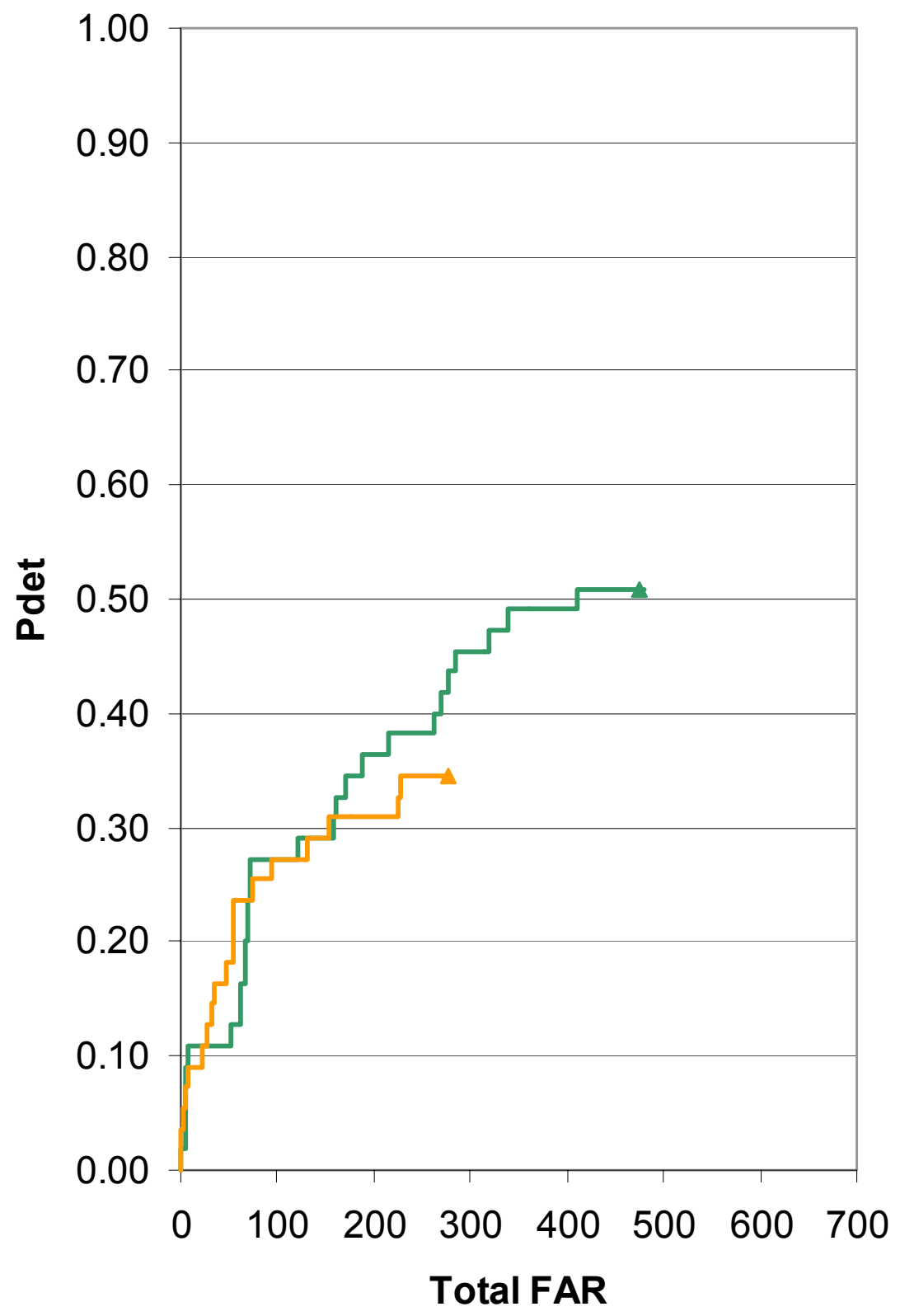

Figure 41. Area $B-P($ det $)$ vs. total $F A R$ within $0.5 \mathrm{~m}$ for demonstrators wo $20 / 40 \mathrm{~mm}$ 


\section{Area C by Demonstrators wo $20 / 40 \mathrm{~mm}$ within $0.5 \mathrm{~m}$}

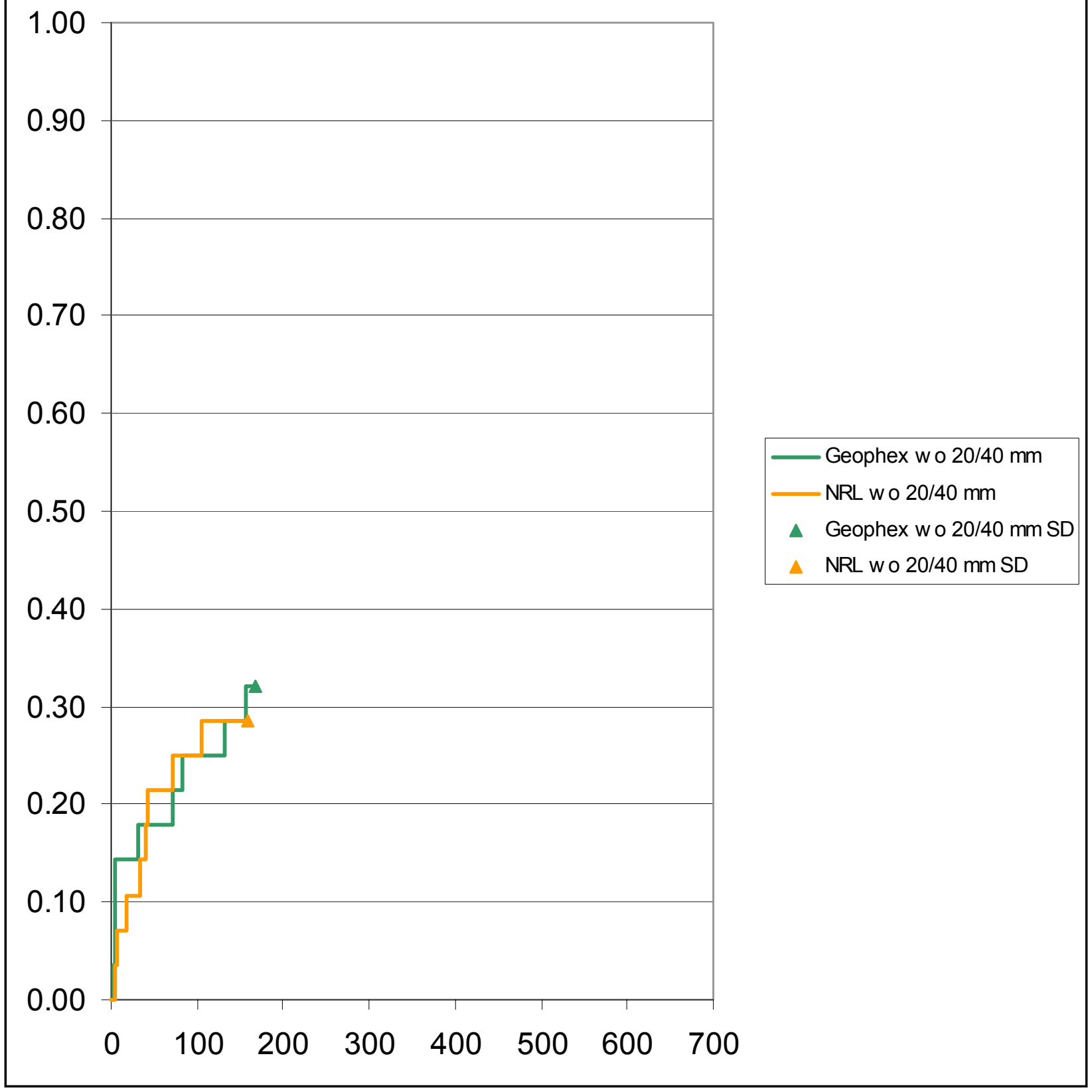

Figure 42. Area $\mathrm{C}-\mathrm{P}(\mathrm{det}) \mathrm{vs}$. total $\mathrm{FAR}$ within $0.5 \mathrm{~m}$ for demonstrators wo $20 / 40 \mathrm{~mm}$ 


\section{Area A wo $20 / 40 \mathrm{~mm}$ by Demonstrators within $0.5 \mathrm{~m}$}

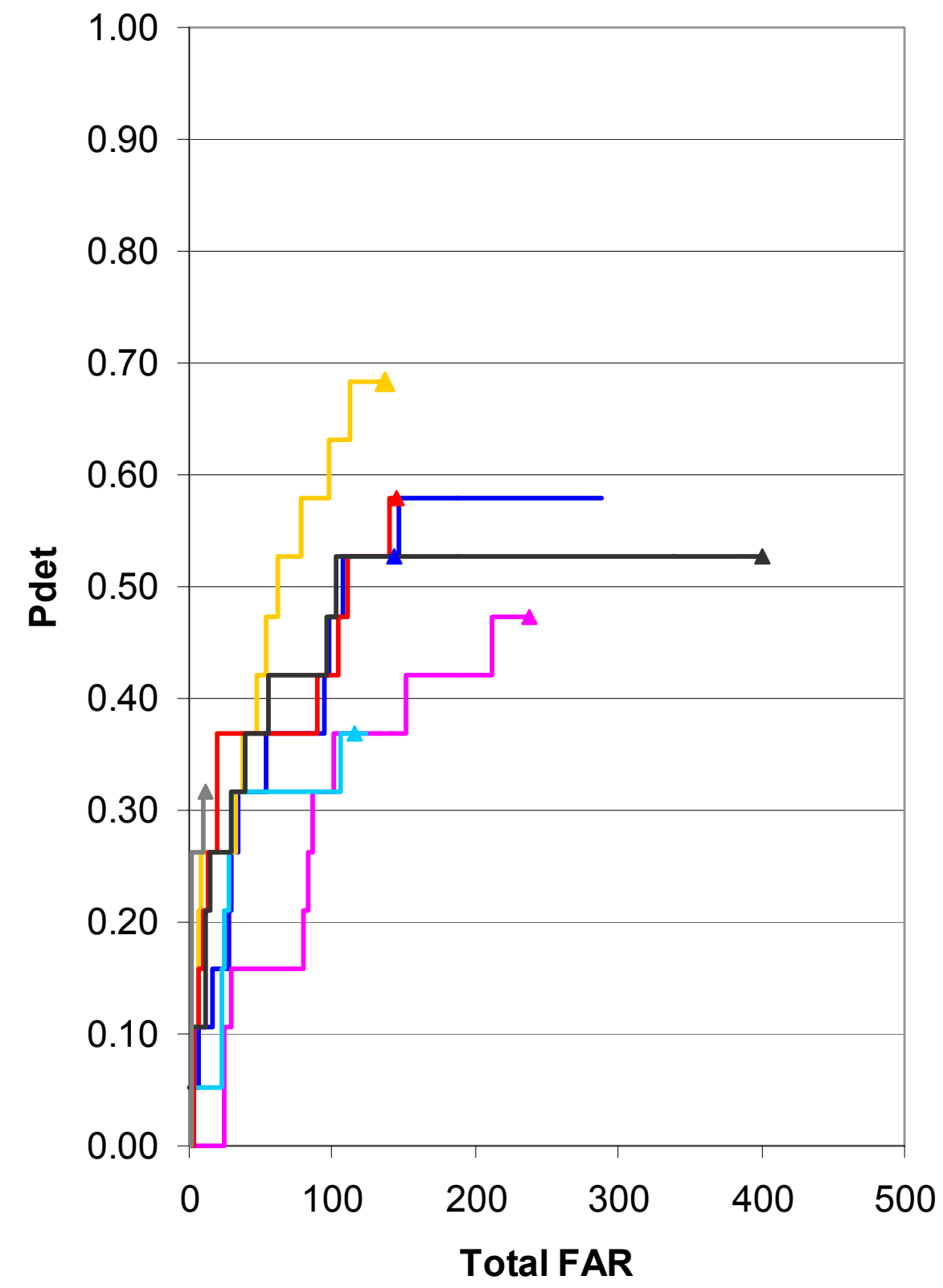

Figure 43. Area A wo $20 / 40 \mathrm{~mm}-\mathrm{P}$ (det) vs. total FAR within $0.5 \mathrm{~m}$ for all demonstrators 


\section{Area B wo $20 / 40 \mathrm{~mm}$ by Demonstrators within $0.5 \mathrm{~m}$}

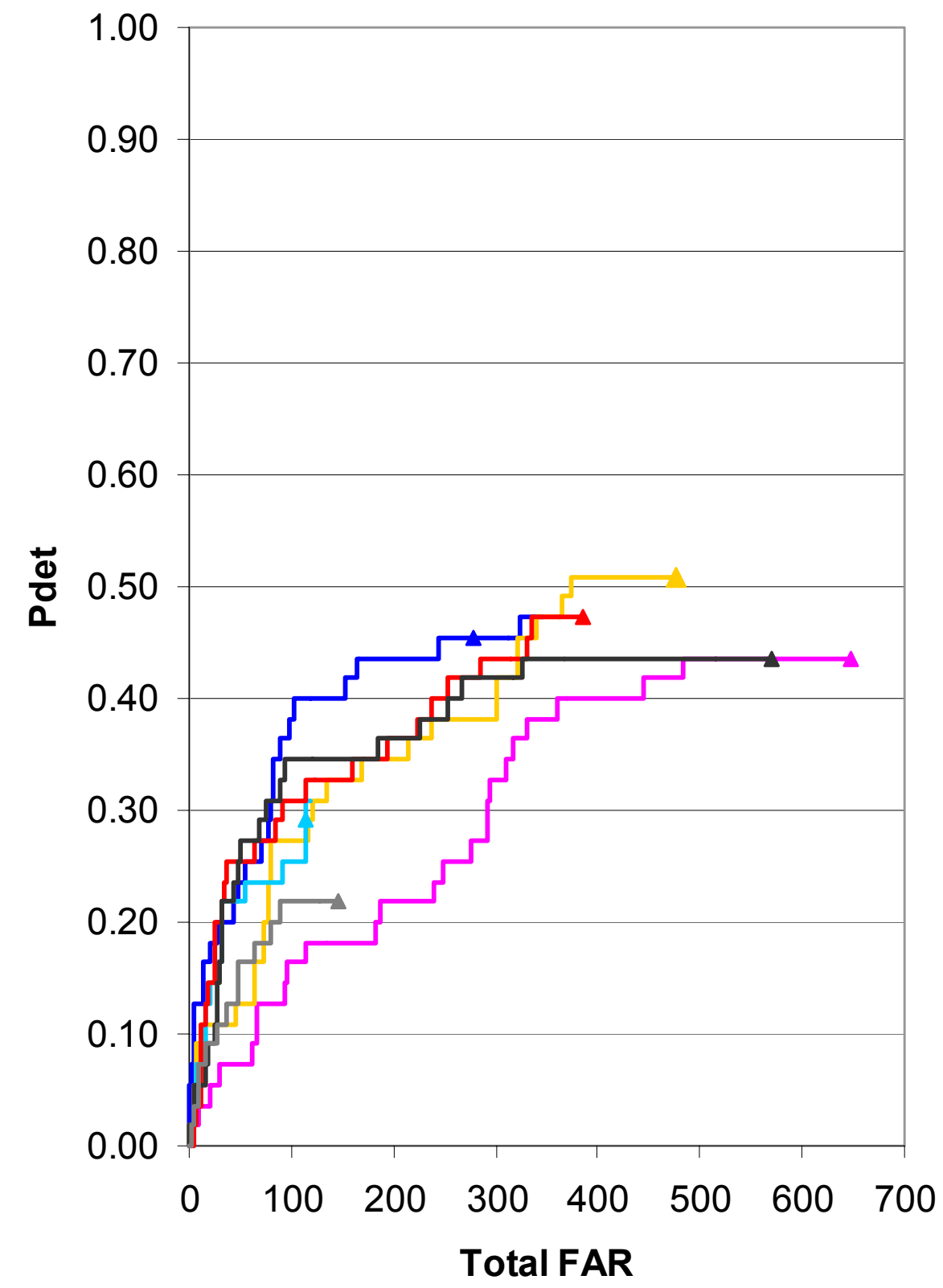

Figure 44. Area $B$ wo $20 / 40 \mathrm{~mm}-\mathrm{P}$ (det) vs. total FAR within $0.5 \mathrm{~m}$ for all demonstrators 


\section{Area C wo $20 / 40 \mathrm{~mm}$ by Demonstrators within $0.5 \mathrm{~m}$}

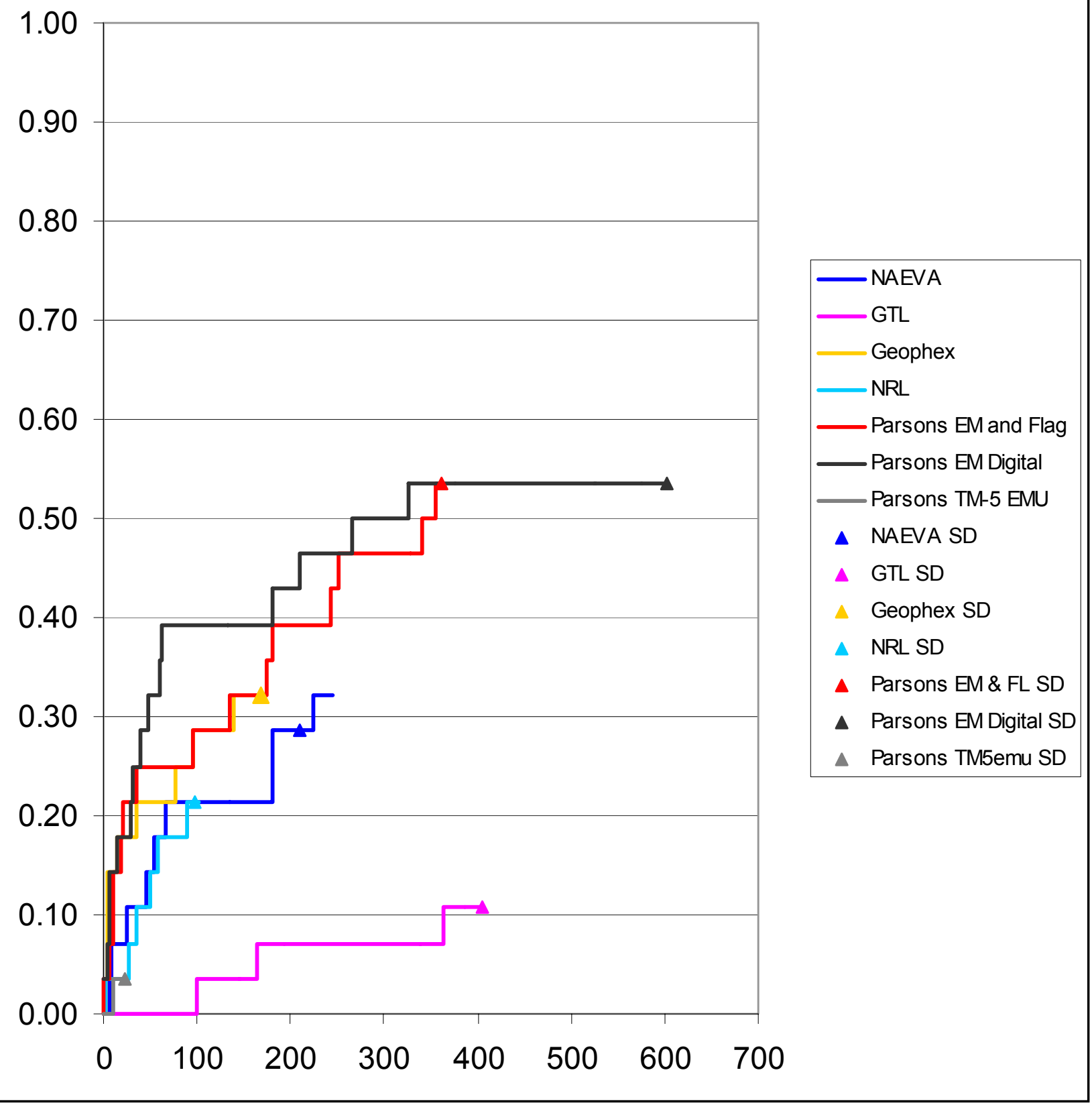

Figure 45. Area $\mathrm{C}$ wo $20 / 40 \mathrm{~mm}-\mathrm{P}$ (det) vs. total $\mathrm{FAR}$ within $0.5 \mathrm{~m}$ for all demonstrators 


\section{Area A by Demonstrators within $0.5 \mathrm{~m}$}

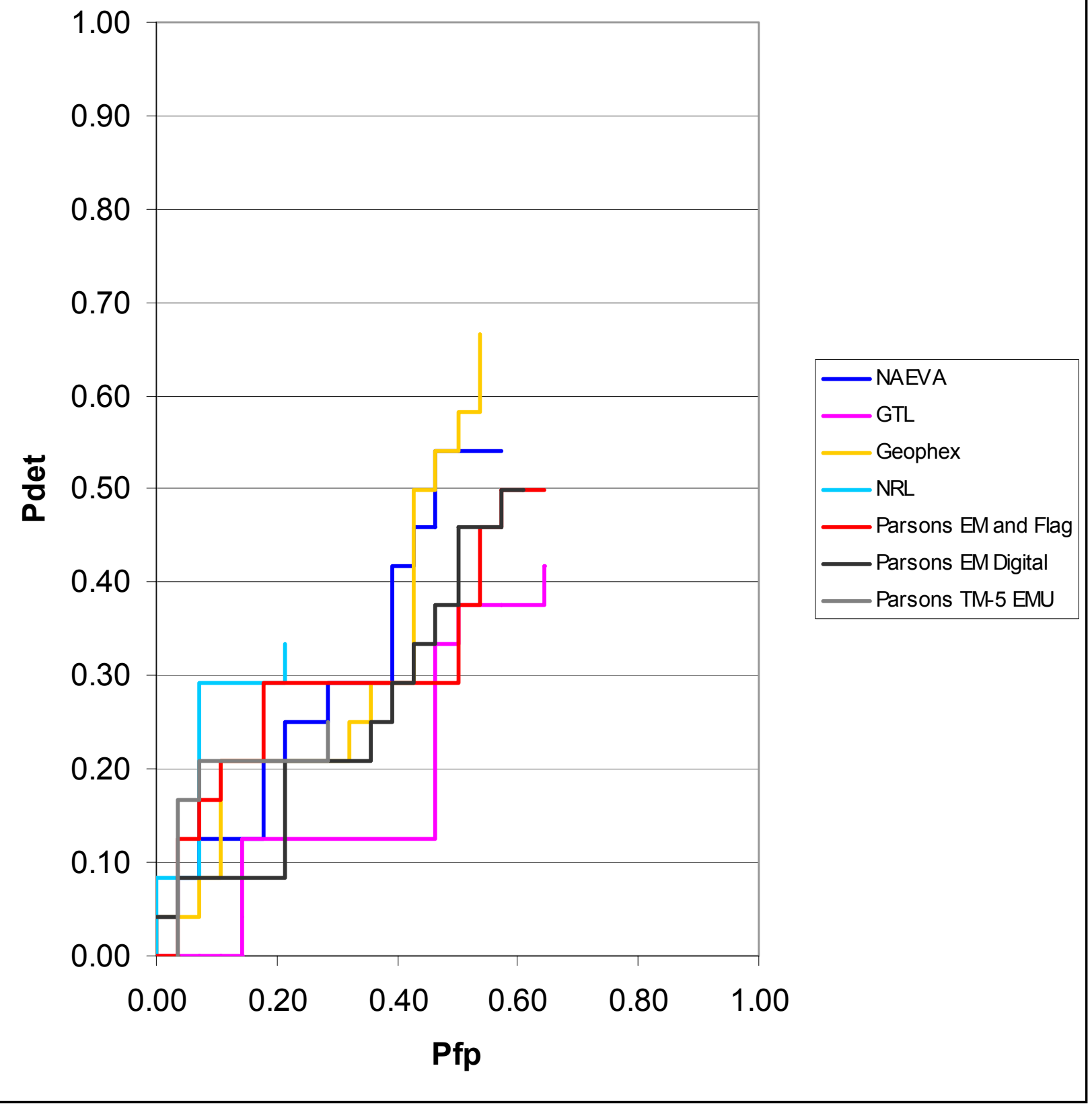

Figure 46. Area $A-P($ det) vs. Pfp within $0.5 \mathrm{~m}$ for all demonstrators 


\section{Area B by Demonstrators within $0.5 \mathrm{~m}$}

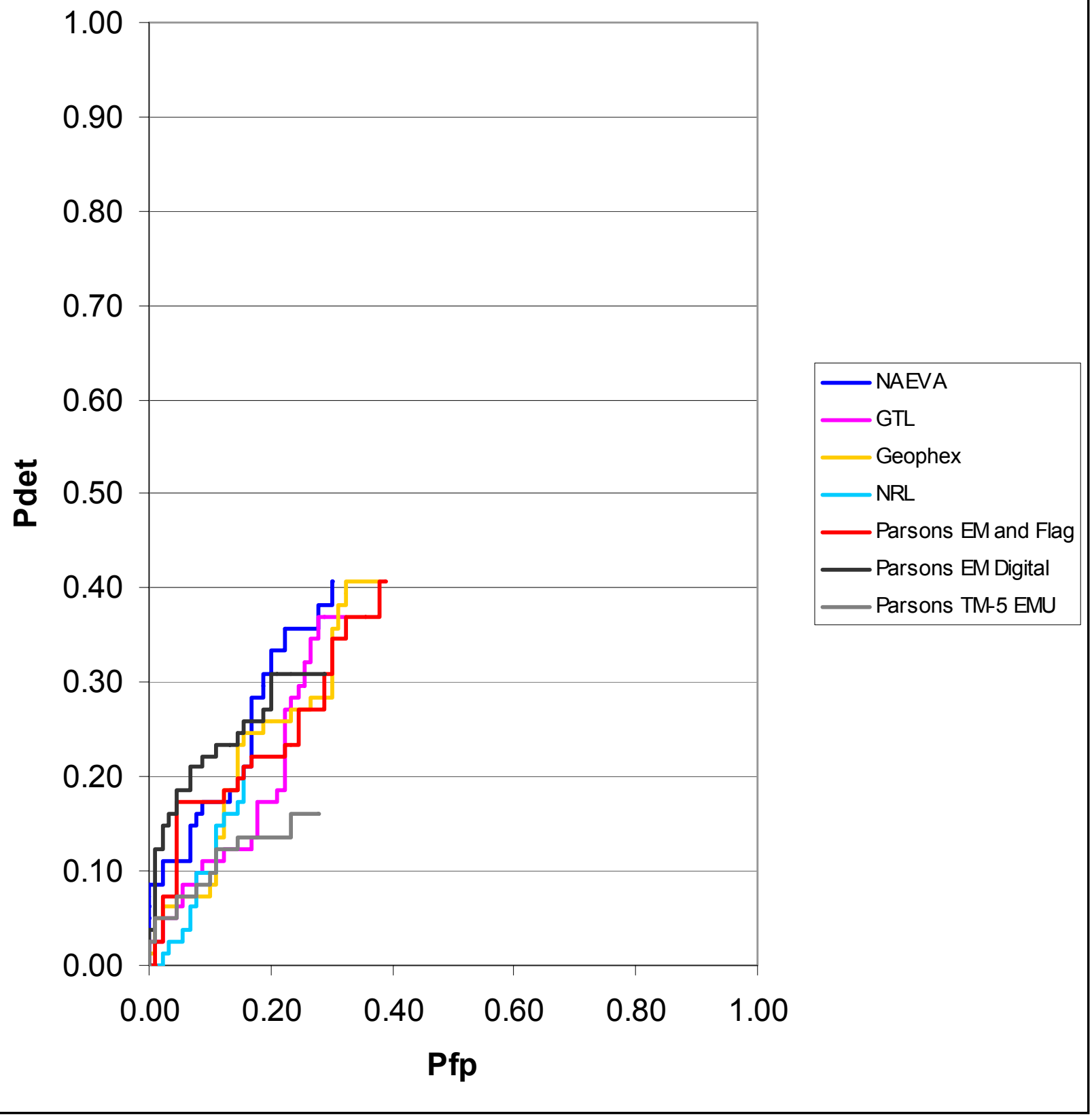

Figure 47. Area $B-P($ det $)$ vs. Pfp within $0.5 \mathrm{~m}$ for all demonstrators 


\section{Area C by Demonstrators within $0.5 \mathrm{~m}$}

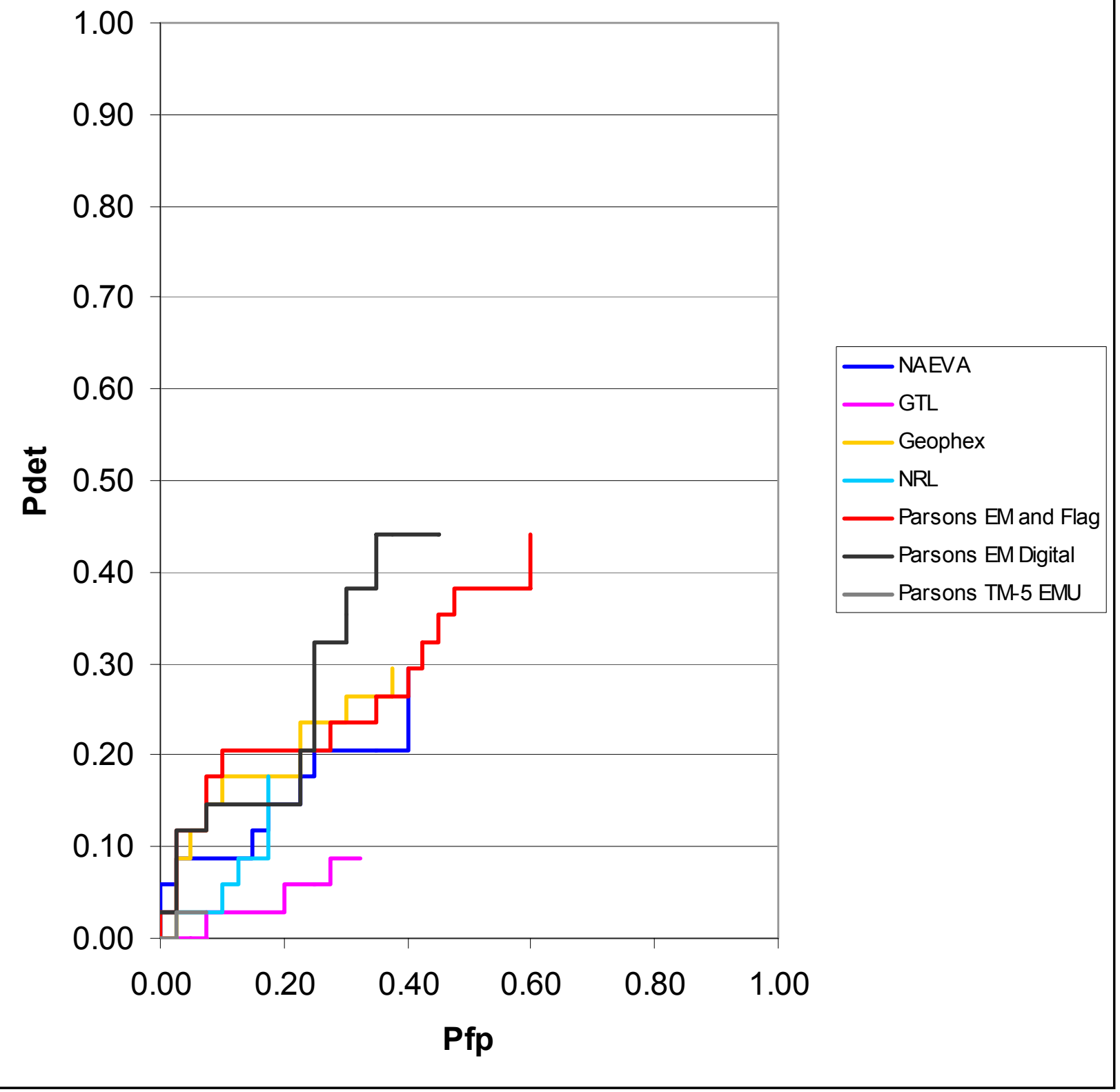

Figure 48. Area $\mathrm{C}-\mathrm{P}(\mathrm{det}) \mathrm{vs.}$ Pfp within $0.5 \mathrm{~m}$ for all demonstrators 


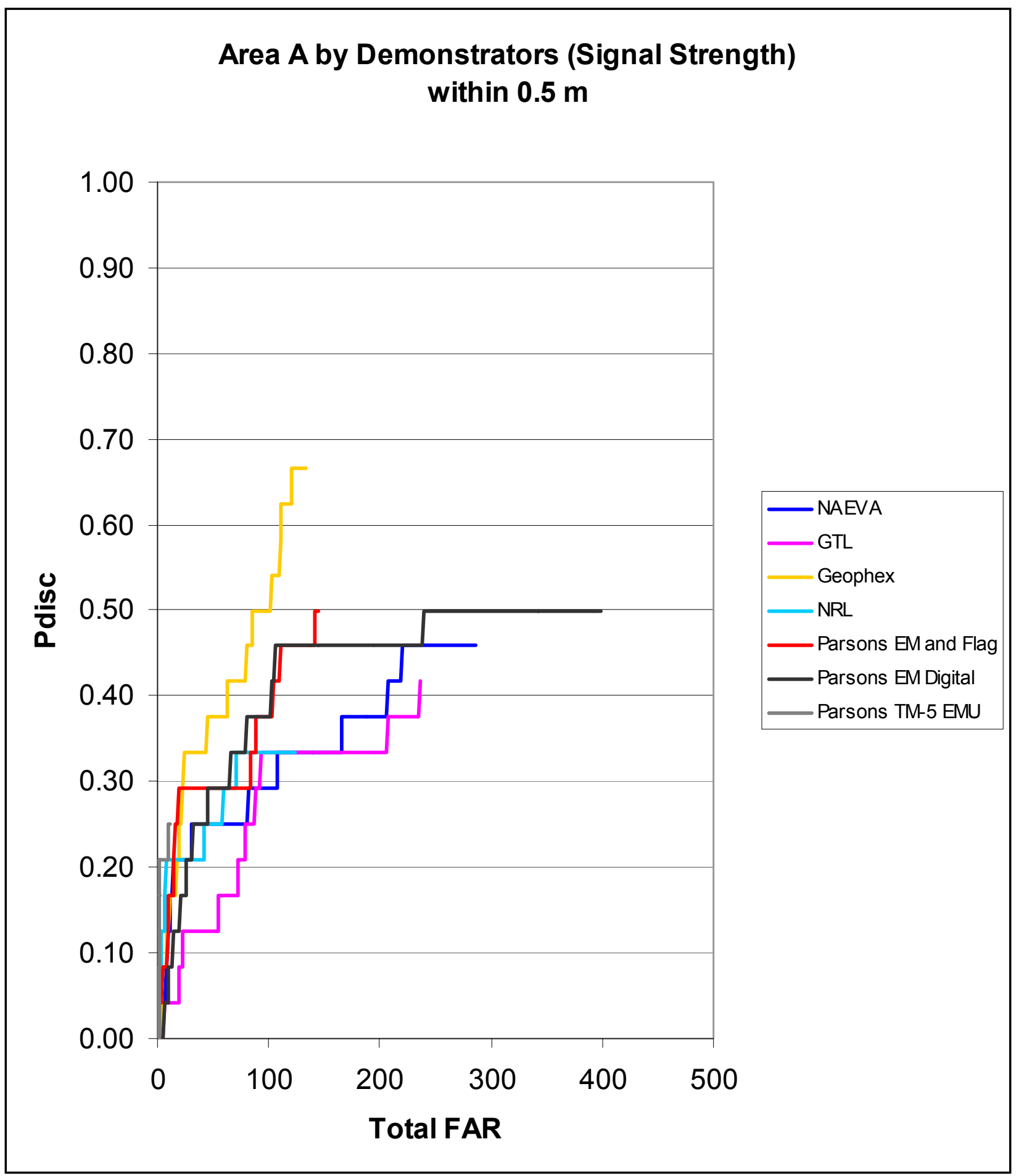

Figure 49. Area A - Pdisc (prioritized by signal strength) vs. total FAR within $0.5 \mathrm{~m}$ for all demonstrators 


\section{Area B by Demonstrators (Signal Strength) within $0.5 \mathrm{~m}$}

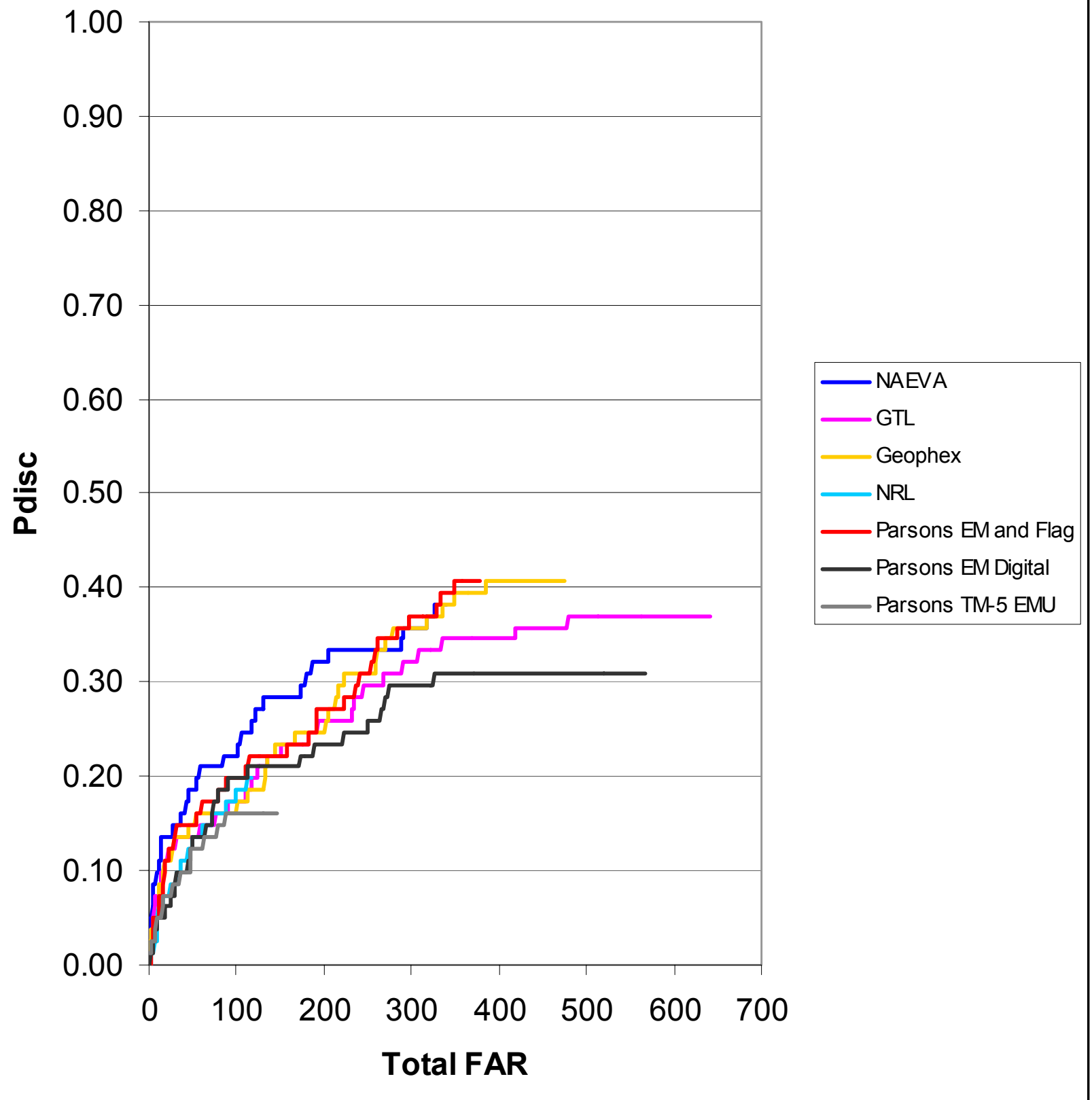

Figure 50. Area B - Pdisc (prioritized by signal strength) vs. total FAR within $0.5 \mathrm{~m}$ for all demonstrators 


\section{Area $\mathrm{C}$ by Demonstrators (Signal Strength) within $0.5 \mathrm{~m}$}

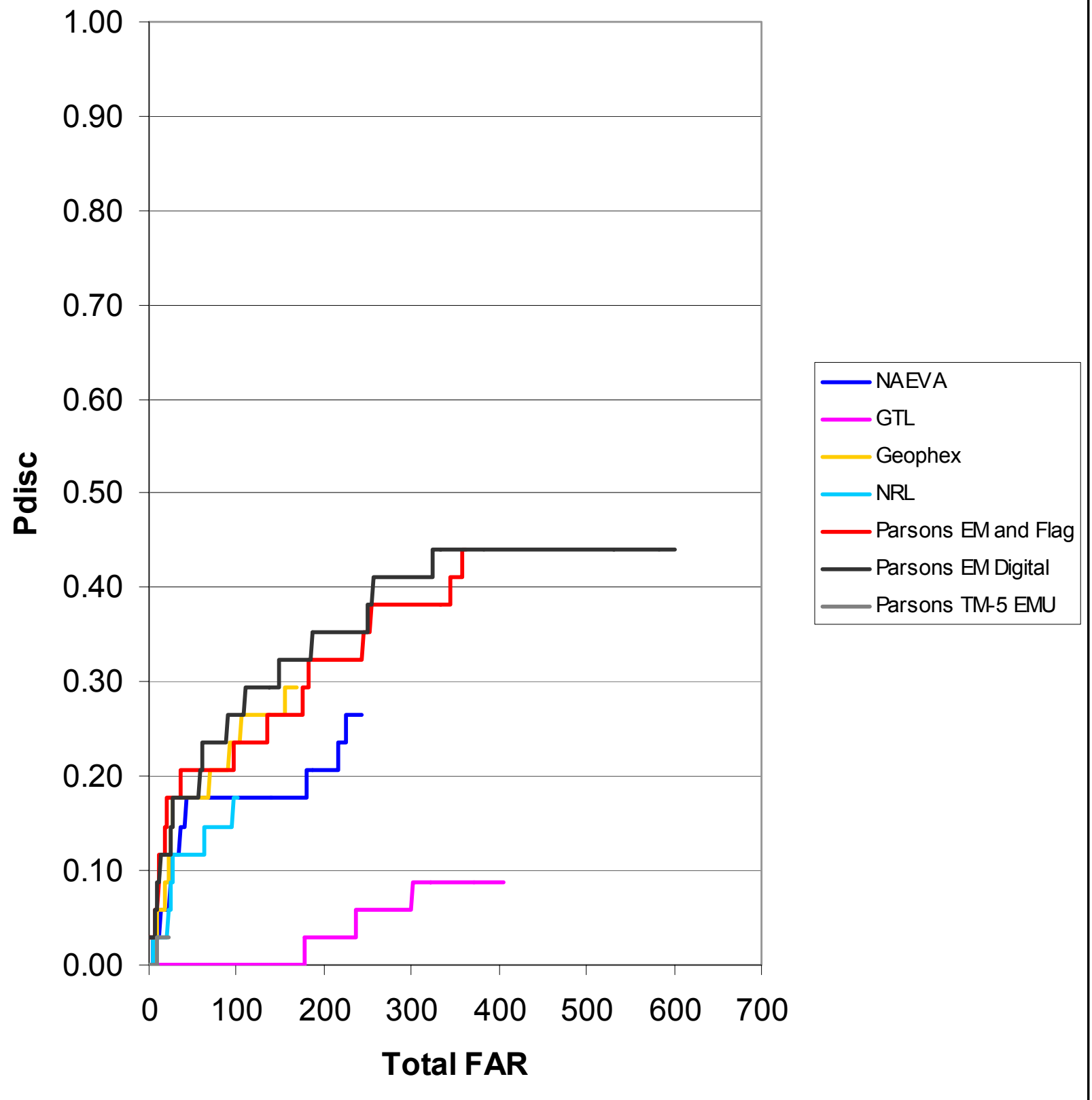

Figure 51. Area $\mathrm{C}-\mathrm{Pdisc}$ (prioritized by signal strength) vs. total FAR within $0.5 \mathrm{~m}$ for all demonstrators 
possible to evaluate the systems' ability to discriminate ordnance from geologic anomalies, because the demonstration area was so cluttered with unknown metallic objects that the effects of geology could not be reliably separated. Some obvious reasons for the decreased overall performance include the facts that, unlike the fairly benign, low noise environment of test sites such as JPG, Kaho'olawe presents an extreme in clutter density, geologic noise, and difficult operating environment.

Overall, the best performances were achieved in Area A where NAEVA, GTL, Geophex, Parson EM and flag, and Parson EM-61 digital had a maximum achievable $\mathrm{P}(\mathrm{det})$ of at least 50 percent when including detections within $0.5 \mathrm{~m}$. Also, the Total FAR was the lowest for all demonstrators in Area A. However, none of the demonstrators reached the $\mathrm{P}(\mathrm{det})$ required for Kaho'olawe Tier II clearance (85 percent). The $\mathrm{P}(\mathrm{det})$ in Areas B and C were significantly lower for all demonstrators and their Total FAR was higher. Most significantly, none of the advanced EMI systems demonstrated significant improved capability over the baseline EM-61 system operated in the "EM and flag" mode. 


\section{Cost Assessment}

\section{Field Cost Performance}

Labor costs associated with each field task were computed by applying the cost factors described in the Demonstration Work Plan and detailed in Table 31. It should also be noted that since all of the demonstrated systems were manportable or handheld, with similar support equipment and capital cost requirements, it was assumed that mobilization/demobilization and life cycle costs would be equal and could be omitted from this relative cost performance evaluation.

Analysis of this table indicates that the field labor costs of most of the demonstrators fall within a fairly narrow range, with the two exceptions being the Parsons TM-5 EMU, which demonstrated significantly, lower costs and NAEVA, which achieved significantly higher costs. These differences may be attributable to the fact that the TM-5EMU survey conducted by Parsons did not appear to adequately cover the required areas (based on their very low number of detected anomalies and on the fact that GTL, using the same sensor, required significantly more time to complete the surveys). A further reason for these differences could be the fact that NAEVA approached this field demonstration effort from a more scientific perspective where one of their primary objectives was to collect very high quality field data to support their ongoing algorithm development efforts. With the exception of NAEVA, all of the other advanced EMI systems compare favorably against the EM-61 "EM and flag" baseline technology.

\section{Weighted Field Cost Performance}

Table 32 summarizes the operational costs of the demonstrator systems after the cost penalties described in Chapter 4 were applied. These penalties consisted of $\$ 200$ for each false alarm (clutter item selected for digging by the demonstrator) and the cost of a complete resurvey for one or more UXO targets missed (not included in the dig list) or erroneously declared as clutter with high confidence. This table highlights the fact that false alarms have (by a large margin) the greatest impact on the cost performance of each system. Table 32 indicates that all seven demonstrators were penalized with the cost of a resurvey at each of the three demonstration areas because their dig lists indicate that UXO would have had been left in the ground. It is difficult to draw conclusions regarding the performance of each system based solely on these results. For example, one may 


\begin{tabular}{|c|c|c|c|c|c|}
\hline \multicolumn{6}{|c|}{$\begin{array}{l}\text { Table } 31 \\
\text { Breakdown of Field Costs }\end{array}$} \\
\hline Demonstrator & $\begin{array}{l}\text { Number of } \\
\text { Operators }\end{array}$ & Categories & Cost & $\begin{array}{l}\text { Time } \\
\text { (hrs, min) }\end{array}$ & Cost to job \\
\hline \multirow[t]{6}{*}{ NAEVA } & 1 & Supervisor & $\$ \$ 95.00$ & $35: 45$ & $\$ \$ 3,396.25$ \\
\hline & 3 & Logistic/Field Setup & 28.50 & $15: 30$ & 441.75 \\
\hline & 3 & Logistic/Field Survey & 28.50 & $83: 00$ & $2,365.50$ \\
\hline & 3 & Logistic/Field Downtime & 28.50 & $1: 30$ & 42.75 \\
\hline & 3 & Logistic/Field Resurvey & 28.50 & $6: 30$ & 185.25 \\
\hline & 4 & Total & & & $\$ 6,431.50$ \\
\hline \multirow[t]{6}{*}{ GTL } & 1 & Supervisor & $\$ 95.00$ & $21: 40$ & $\$ 2,058.33$ \\
\hline & 1 & Logistic/Field Setup & 28.50 & $3: 45$ & 106.88 \\
\hline & 1 & Logistic/Field Survey & 28.50 & $13: 43$ & 390.92 \\
\hline & 1 & Logistic/Field Downtime & 28.50 & $3: 34$ & 101.65 \\
\hline & 1 & Logistic/Field Resurvey & 28.50 & & \\
\hline & 2 & Total & & & $\$ 2,657.78$ \\
\hline \multirow[t]{6}{*}{ Geophex } & 1 & Supervisor & $\$ 95.00$ & $24: 30$ & $\$ 2,327.50$ \\
\hline & 2 & Logistic/Field Setup & 28.50 & $10: 30$ & 299.25 \\
\hline & 2 & Logistic/Field Survey & 28.50 & $24: 25$ & 688.75 \\
\hline & 2 & Logistic/Field Downtime & 28.50 & $2: 20$ & 66.50 \\
\hline & 2 & Logistic/Field Resurvey & 28.50 & $2: 55$ & 83.12 \\
\hline & 3 & Total & & & $\$ 3,465.12$ \\
\hline \multirow[t]{6}{*}{ NRL } & 1 & Supervisor & $\$ 95.00$ & $20: 02$ & $\$ 1,903.17$ \\
\hline & 3 & Logistic/Field Setup & 28.50 & $9: 00$ & 256.50 \\
\hline & 3 & Logistic/Field Survey & 28.50 & $34: 06$ & 977.55 \\
\hline & 3 & Logistic/Field Downtime & 28.50 & 19:00 & 541.50 \\
\hline & 3 & Logistic/Field Resurvey & 28.50 & & \\
\hline & 4 & Total & & & $\$ 3,678.72$ \\
\hline \multirow{6}{*}{$\begin{array}{l}\text { Parson (EM-61) } \\
\text { EM and Flag }\end{array}$} & 1 & Supervisor & $\$ 95.00$ & $28: 01$ & $\$ 2,661.58$ \\
\hline & 2 & Logistic/Field Setup & 28.50 & $18: 57$ & 540.08 \\
\hline & 2 & Logistic/Field Survey & 28.50 & $58: 30$ & $1,667.25$ \\
\hline & 2 & Logistic/Field Downtime & 28.50 & $17: 00$ & 484.50 \\
\hline & 2 & Logistic/Field Resurvey & 28.50 & & \\
\hline & 3 & Total & & & $\$ 5,353.41$ \\
\hline \multirow{6}{*}{$\begin{array}{l}\text { Parson EM-61 } \\
\text { Digital }\end{array}$} & 1 & Supervisor & $\$ 95.00$ & $17: 55$ & $\$ 1,702.08$ \\
\hline & \begin{tabular}{|l|}
1 \\
\end{tabular} & Logistic/Field Setup & 28.50 & $16: 44$ & 476.60 \\
\hline & 1 & Logistic/Field Survey & 28.50 & $15: 04$ & 446.50 \\
\hline & 1 & Logistic/Field Downtime & 28.50 & $7: 16$ & 207.10 \\
\hline & 1 & Logistic/Field Resurvey & 28.50 & $2: 50$ & 80.75 \\
\hline & 2 & Total & & & $\$ 2,913.33$ \\
\hline \multirow{6}{*}{$\begin{array}{l}\text { Parson TM-5 } \\
\text { EMU }\end{array}$} & 1 & Supervisor & $\$ 95.00$ & $6: 56$ & $\$ 658.67$ \\
\hline & 3 & Logistic/Field Setup & 28.50 & $12: 10$ & 345.75 \\
\hline & 3 & Logistic/Field Survey & 28.50 & $5: 00$ & 142.50 \\
\hline & 3 & Logistic/Field Downtime & 28.50 & $5: 30$ & 156.75 \\
\hline & 3 & Logistic/Field Resurvey & 28.50 & & \\
\hline & 4 & Total & & & $\$ 1,304.67$ \\
\hline
\end{tabular}




\begin{tabular}{|c|c|c|c|c|c|c|c|}
\hline \multicolumn{8}{|c|}{\begin{tabular}{|l} 
Table 32 \\
Demonstrator Costs Including Penalties for False Alarms and for \\
Leaving UXO Targets in Ground
\end{tabular}} \\
\hline & NAEVA & GTL & Geophex & NRL & $\begin{array}{l}\text { Parsons } \\
\text { (EM-61) } \\
\text { (EM and } \\
\text { Flag }\end{array}$ & \begin{tabular}{|l|} 
Parsons \\
TM-5 EMU
\end{tabular} & \begin{tabular}{|l} 
Parsons \\
Digital
\end{tabular} \\
\hline $\begin{array}{l}\text { Cost to } \\
\text { Survey }\end{array}$ & $\$ \quad 2,366$ & 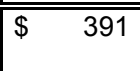 & $\begin{array}{ll}\$ & 689\end{array}$ & 978 & \$ \$ 1,667 & 143 & $4 \quad 447$ \\
\hline \begin{tabular}{|l|} 
Cost of \\
Resurvey
\end{tabular} & 2,366 & 391 & 689 & 978 & 1,667 & 143 & 447 \\
\hline \begin{tabular}{|l|} 
Cost of \\
False \\
Alarms \\
\end{tabular} & 128,000 & 260,600 & 157,800 & 123,600 & 176,200 & 43,600 & 319,400 \\
\hline Total Cost & \begin{tabular}{|l|}
$\$ 132,732$ \\
\end{tabular} & \begin{tabular}{|l|}
$\$ 261,382$ \\
\end{tabular} & \begin{tabular}{|l|}
$\$ 159,178$ \\
\end{tabular} & $\$ 125,556$ & \begin{tabular}{|l|}
$\$ 179,534$ \\
\end{tabular} & $\$ 43,886$ & $\$ 320,294$ \\
\hline
\end{tabular}

conclude that the Parson TM-5 EMU system is superior because of its significantly lower costs, but analysis of their detection performance would show that it failed to detect a very large percentage of the UXO present. Since the cost of false alarms dominates this type of analysis in very highly cluttered areas such as Kaho'olawe, any system could achieve low costs by operating on a very low point on the ROC curve. Thus, any cost comparisons between systems have to be correlated with their respective ROC curve performance in order to reach reasonable conclusions. Assuming that the systems are operated at reasonable points of the ROC curve, Table 31 would indicate that Parsons EM-61 EM and flag and NAEVA are the most cost-effective performers. Another factor to be considered in future evaluations is the fact that the $\$ 200$ cost per false alarm is excessive for sites such as Kaho'olawe where the high density and fairly shallow depths of most metallic clutter from munitions fragments allows for rapid removal with minimal manpower requirements. 


\section{Regulatory Issues - Compliance and Acceptance}

Members of the regulatory community who are aware of these technology demonstrations are listed in Appendix A. 


\section{Technology Transition/ Implementation}

The next step in this effort consists of the preparation of self-evaluation reports by each of the demonstrators and preparation of the Project Final Report by the Government. These reports, together with implementation recommendations will be forwarded to the Navy Facilities Command (NAVFAC) and Parsons/UXB personnel currently managing and conducting UXO remediation operations at Kaho'olawe. Mr. Jim Putnam, (808) 474-0559, extension 224, is the project manager for NAVFAC.

The planned schedule for remaining activities under this ESTCP-funded project are as follows:

FY02 Milestones................................................ Est. Completion

a. Complete Demonstrator Self-Evaluation Reports......................... 06/30/02

b. Complete Project Final Report................................................... 08/31/02 


\section{Lessons Learned}

The most surprising conclusion from this demonstration was the fact that none of the advanced EMI systems demonstrated significant performance and/or cost improvements over the baseline technology consisting of a standard EM-61 system operated in an "EM and Flag" mode. The relatively good performance achieved by this system in this mode would indicate that there may be advantages to providing real-time feedback to the UXO survey crews so that they may collect additional sensor data (in orthogonal directions) over suspected anomalies, rather than blindly surveying lanes with fixed lane widths and sampling rates. Such a survey method would also allow the crew to visually identify and mark surface anomalies that could otherwise be misinterpreted as UXO during the postsurvey data analysis.

Another important lesson learned from this demonstration is the difficulty in setting up test sites and conducting demonstrations at live UXO sites that are in the process of being remediated. Even though the calibration and demonstration areas had been cleared numerous times, there were still excessive amounts of metallic clutter from unknown sources and even live ordnance that remained in these areas. As a result, the accuracy of the groundtruth available for such test sites is always in doubt. In addition, the presence of unknown metallic clutter prevented the evaluation of the advanced EMI systems' assumed capability to mitigate the effects of geologic noise. Finally, the safety and logistics problems associated with conducting technology demonstrations concurrent with actual UXO cleanup operations proved to be a very inefficient, costly, and time consuming process.

It can be concluded from these demonstrations that additional research, development, and demonstration work is needed in order to produce UXO technologies that meet reasonable detection, discrimination, and false alarm performance goals, especially in difficult sites such as those encountered at Kaho'olawe. 


\section{References}

Cespedes, E. R. (2001). "Advanced UXO detection/discrimination technology demonstration - U.S. Army Jefferson Proving Ground, Madison, Indiana," Technical Report ERDC/EL TR-01-20, U.S. Army Engineer Research and Development Center, Vicksburg, MS.

Geonics Limited. (1999). "EM-63 full time domain electromagnetic UXO detector operating instructions," Ontario, Canada.

(2000). "EM61 metal detector for unexploded ordnance (UXO) detection and characterization," Ontario, Canada.

Geophysical Technology Limited. (2002). "Requested information regarding the GTL TM-5 EMU," Commercial-In-Confidence correspondence to the U.S. Army Corps of Engineers.

Naval Research Laboratory. (2001). "Man-portable adjuncts for the MTADS," Technical Report NRL/PU/6110-01-434, Washington DC.

Naval Explosive Ordnance Disposal Technology Division (NAVEODTECHDIV). (2001a). "Technology demonstration plan Advanced UXO detection/discrimination technology demonstration, Kaho'olawe Island, Hawaii," unpublished.

(2001b). "Site preparation plan - Advanced UXO detection/ discrimination technology demonstration, Kaho'olawe Island, Hawaii," unpublished.

U.S. Army Environmental Center and Naval Explosive Ordnance Disposal Technology Division. (1997). "UXO technology demonstration program at Jefferson Proving Ground, Phase III," Report No. SFIM-AEC-ET-CR-97011.

Won, I. J., Keiswetter, K., and Novikova, E. (1998). "Electromagnetic induction spectroscopy," Journal of Environmental and Engineering Geophysics 3(1), $27-40$. 


\section{Appendix A Points of Contact}

\section{The NAVEODTECHDIV POC is:}

Mr. Hien Dinh NAVEODTECHDIV

2008 Stump Neck Road

Indian Head, MD 20640-5070

Phone: (301) 7446850 ext. 267

FAX: (301) 744-6947

E-mail:dinh@eodpoe2.navsea.navy.mil

The ERDC POC is:

Dr. Ernesto Cespedes

U.S. Army Engineer Research and Development Center 3909 Halls Ferry Road

Vicksburg, MS 39180-6199

Phone: (601) 634-2655

FAX: (601) 634-2732

E-mail: cespede@wes.army.mil

\section{The AEC POC is:}

Mr. George Robitaille

U.S. Army Environmental Center

ATTN: SFIM-AEC-P2/ETD

Bldg E4430

Aberdeen Proving Ground, MD 21010-5401

Phone: (410) 436-6850

FAX: (410) 436-6836

E-mail: George.Robitaille@aec.apgea.army.mil 
Important points of contact in the regulatory and user community who have knowledge of the demonstration include:

\section{Interstate Technology Regulatory Cooperation (ITRC)}

\section{ITRC UXO WORKGROUP}

\section{Workgroup Co-Leaders}

Jim Austreng (Team Co-Lead)

California EPA

Department of Toxic Substances Control

10151 Croydon Way, Suite 3

Sacramento, CA 95827-2106

P: 916-255-3702

jaustren@dtsc.ca.gov

Jennifer Roberts (Team Co-Lead)

Alaska Department of Environmental Conservation

555 Cordova Street

Anchorage, AK 99501

P: 907-269-7553

Jennifer_Roberts@envircon.state.ak.us

\section{Workgroup Members}

David Asiello

U.S. Department of Defense

3400 Defense Pentagon

Washington, DC 20301-3400

P: 703-697-7363

F: 703-695-4981

asielldj@acq.osd.mil

Tim Bahr

Florida Department of Environmental Protection

2600 Blair Stone Road

Tallahassee, FL 32399-2400

P: 850-921-9984

F: 850-922-4939

Tim.Bahr@dep.state.fl.us

Geoff Cullison

Office of the Chief of Naval Operations

Navy Environmental Restoration Program

2211 South Clark Street

Arlington, VA 22202-3735

P: 703-602-5329

F: 703-602-2676

cullison.geoffrey@hq.navy.mil 
Jeff Edson

Colorado Department of Public Health \& Environment

4300 Cherry Creek Drive, South

Denver, CO 80246

P: 303-692-3388

F: 303-759-5355

Jeff.edson@state.co.us

Dwight Hempel

Department of Interior

Bureau of Land Management

1849 C Street, NW, MS 1000LS

Washington, DC 20240

P: 202-452-7778

F: $202-452-7708$

dwight_hempel@blm.gov

Aimee Houghton

Center for Public Environmental Oversight

122 C Street NW, Suite 700

Washington, DC 20001-2109

P: 202-662-1888

F: 202-628-1825

aimeeh@cpeo.org

Dave Larsen

Utah Department of Environmental Quality

288 N. 1460 West

Salt Lake City, UT 84116

P: 801-538-6749

F: 801-538-6715

Dlarsen@deq.state.ut.us

Mike Liberati

DuPont Corporate Remediation Group

2000 Cannonball Road

Pompton Lakes, NJ 07442

P: 302-892-7421

michael.r.liberati@usa.dupont.com

Chris Maurer

Washington Department of Ecology

P.O. Box 47600

Olympia, WA 98504

P: 360-407-7223

Cmau461@ecy.wa.gov

Marshall Nay

TRW

6001 Indian School Road

Albuquerque, NM 87110

P: 505-998-8359

F: 505-998-8125

marshall.nay@trw.com 
Eric Noack

Nevada Division of Environmental Protection

333 Nye Lane, ES-111

Carson City, NV 89710

P: 775-687-4670 x3032

enoack@ndep.carson-city.nv.us

Steve Nussbaum

Illinois Environmental Protection Agency

1021 N. Grand Ave. East

Springfield, IL 62794-9276

P: 217-782-9803

F: 217-524-3291

epa4129@epa.state.il.us

James Hersey

Joint UXO Coordination Office

Attn: AMSEL-RD-UXO-CO

10221 Burbeck Road

Ft. Belvoir, VA 22060-5806

P: 703-704-2609

F: 703 704-2074

jhersey@nvl.army.mil

Rodney Sobin

Virginia Department of Environmental Quality

629 East Main Street

Richmond, VA 23233

P: 804-698-4382

F: 804-698-4264

rsobin@deq.state.va.us

Jerry Stamps

Environmental Engineer

South Carolina Department of Health and Environmental Conservation

2600 Bull Street

Columbia, SC 29201

P: 803-896-4285

F: 803-896-4002

stampsjm@columb34.dhec.state.sc.us

Philip Stroud

Alabama Department of Environmental Management

P.O. Box 301463

Montgomery, AL 36130

P: 334-271-7750

F: $334-279-3050$

pns@adem.state.al.us

Jeff Swanson

Colorado Department of Health and the Environment, Hazardous Materials

Division

4300 Cherry Creek Drive, South

Denver, CO 80246-1530

P: 303-692-3416

F: 303-759-5355

Jeffrey.swanson@state.co.us 
Julie Wanslow

New Mexico Department of the Environment

Harold S. Runnels Building

1190 St. Francis Dr.

Santa Fe, NM 87505-4182

P: 505-827-1536

julie_wanslow@nmenv.state.nm.us

Greg Zalaskus

New Jersey Department of Environmental Protection

P.O. Box 028

401 East State Street

Trenton, NJ 08625-0413

P: 609-984-2065

.F: 609-633-1545

gzalasku@dep.state.nj.us 


\section{Appendix B Data Archiving}

This appendix contains digital archive directory/subdirectory information about the sensor data, maps, and results by each demonstrator for the Advanced UXO Detection/Discrimination Technology Demonstration, Kaho'olawe, Hawaii. The Technology Demonstration Plan, the Site Preparation Plan, and the Geophysical Background Survey report are contained in the root directory of the digital archive. To obtain a copy of this archive, a written request must be submitted to one of the authors of this report.

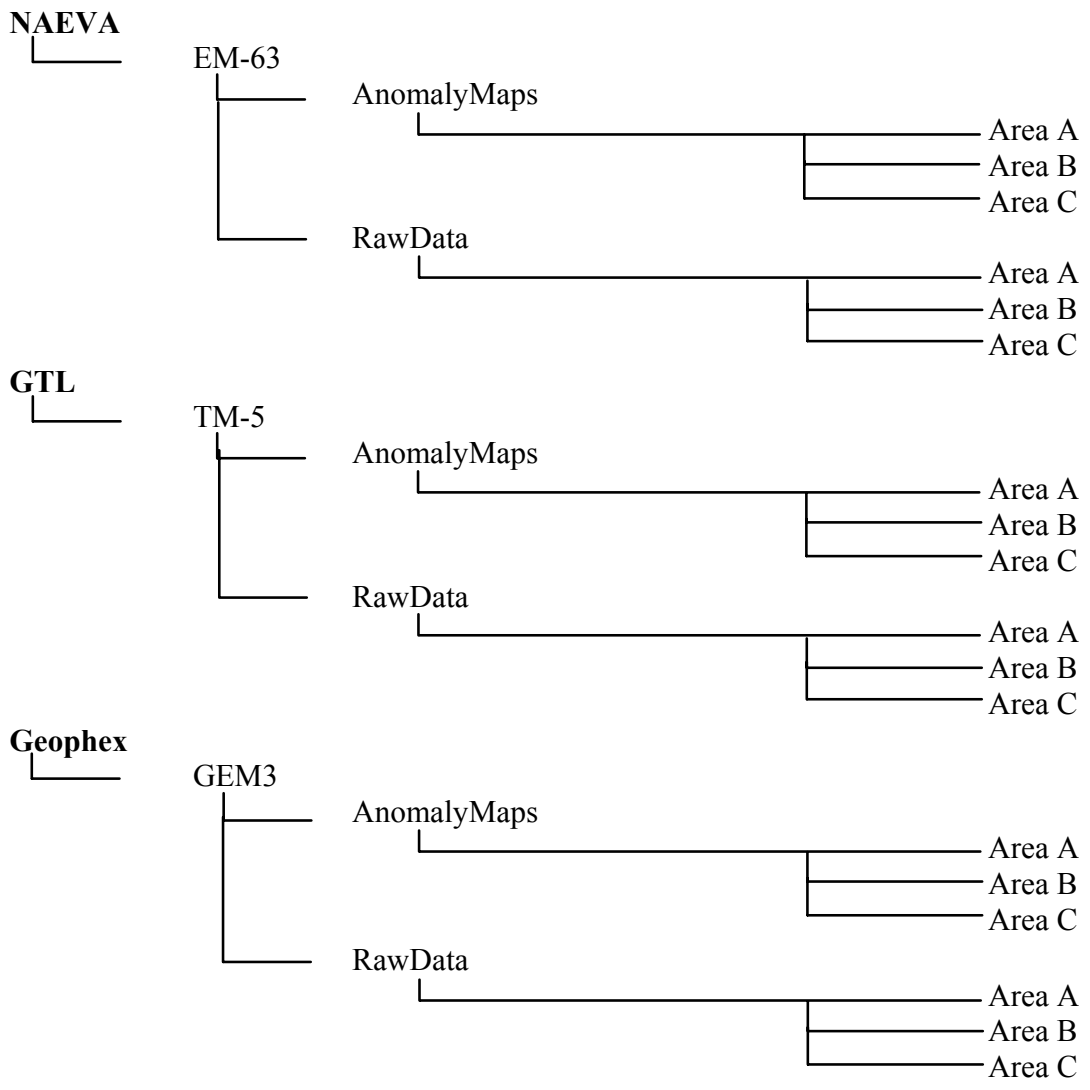

Directory map (continued on next page) 
Directory map (continued)

NRL
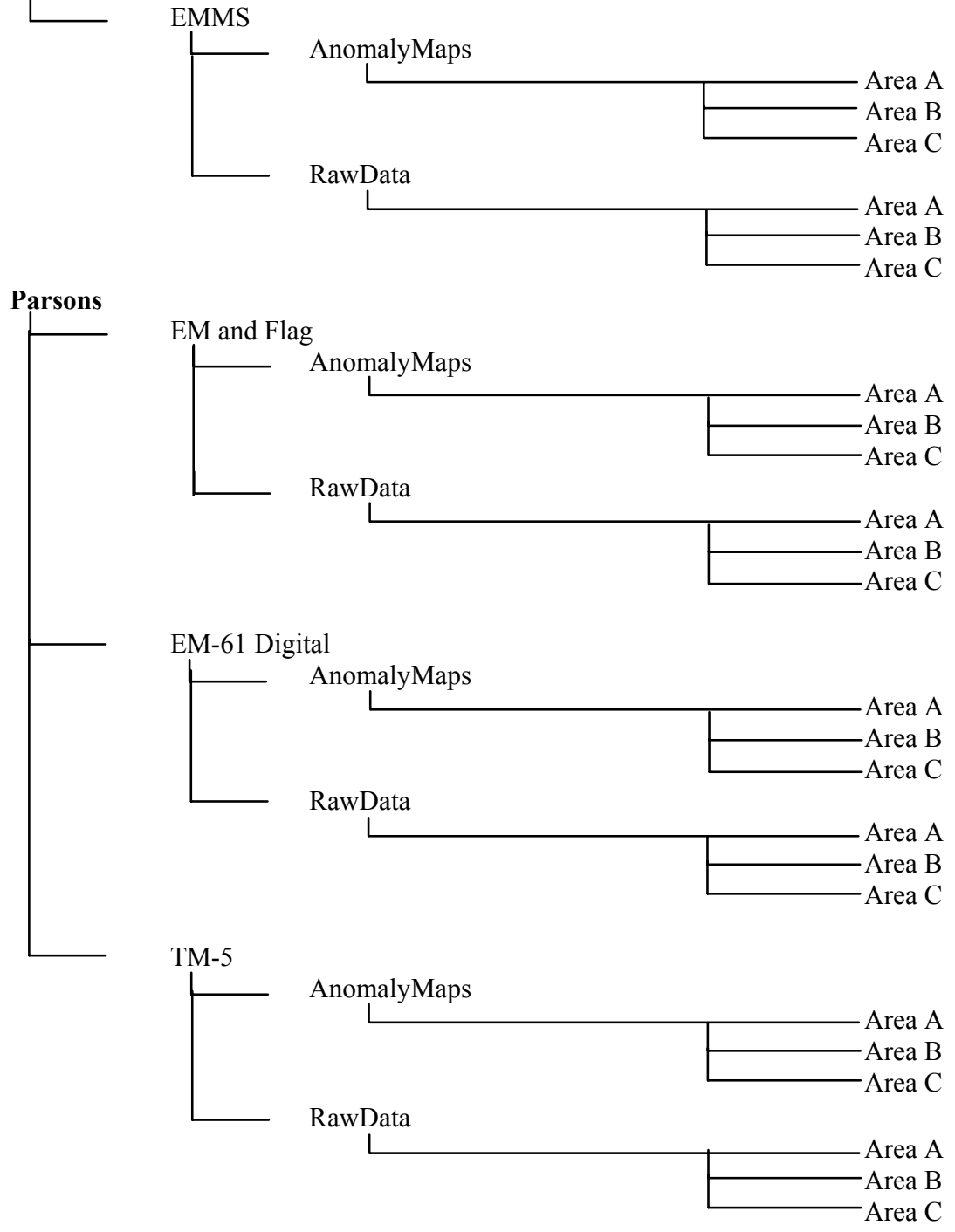

Directory

AnomalyMaps

RawData

\section{Description}

Image of demonstrators' anomaly maps, each area is in its own subdirectory

Raw data for each area collected by demonstrator 


\section{Appendix C Evaluation of Data at $1.0 \mathrm{~m}$}

This Appendix contains the same analysis done in the report using a radius of $1.0 \mathrm{~m}$ instead of the $0.5 \mathrm{~m}$.

\section{Performance Data}

In accordance with the Demonstration Plan, each of the demonstrators was responsible for determining the best method of employing his system in order to:

a. Ensure full coverage of each demonstration area.

$b$. Collect high-quality sensor data to support detection and discrimination requirements.

c. Achieve high production rates.

d. Minimize man-hour requirements and costs.

All demonstrators were able to complete the field surveys within the allotted time periods. There was a wide range in the demonstrators' survey data quality, data density, quality of analysis, and compliance with the data submission requirements specified in the Demonstration Plan (NAVEODTECHDIV 2001a). ${ }^{1}$ For example, a number of demonstrators failed to include required dig list information such as recommended stop dig point, appropriate confidence levels, and signal strength levels, and most demonstrators failed to reanalyze their data and prepare dig lists that excluded the small 20- and 40-mm targets. This lack of adherence to the requirements of the Demonstration Plan has made the interpretation of results and adequate across-demonstrator performance comparisons very difficult. This appendix presents a summary of the data submitted by the demonstrators and the Government's assessment of their performance.

It should be noted that because of the very high numbers of false alarms submitted by all demonstrators, the Government was not able to fully investigate the sources of all of them. Nevertheless, during April 2002, NAVEODTECHDIV personnel conducted extensive surveys and excavation activities in the calibration and demonstration areas in order to verify the emplaced target locations and

1 Reference information follows the main text. 
to attempt to identify the sources of a large percentage of the false alarms. Information from these postdemonstration activities was incorporated into the groundtruth data used to evaluate the demonstrators' performance.

\section{Detection results}

Table $\mathrm{C} 1$ summarizes the number of UXO targets detected within a 1.0-m circular error by each demonstrator. The maximum achievable probability of detection, $\mathrm{P}(\mathrm{det})$, is also given in the table. The $\mathrm{P}(\mathrm{det})$ is calculated as the number of declared items that correspond to emplaced UXO targets (even though they may have been misclassified as clutter) divided by the actual number of UXO targets emplaced in the demonstration site. Table $\mathrm{C} 2$ summarizes the detection results achieved when the small UXO targets (20- and 40-mm projectiles) were excluded from the evaluation.

\begin{tabular}{|c|c|c|c|c|c|}
\hline \multicolumn{6}{|c|}{\begin{tabular}{|l} 
Table C1 \\
P(det) by Area within $1.0 \mathrm{~m}$ \\
\end{tabular}} \\
\hline & & \multicolumn{3}{|c|}{ Within $1.0 \mathrm{~m}$} & \multirow[b]{2}{*}{ Total } \\
\hline & & Area A & Area B & Area C & \\
\hline & \begin{tabular}{|l|}
$\begin{array}{l}\text { Number of Actual } \\
\text { Targets }\end{array}$ \\
\end{tabular} & 24 & 81 & 34 & 139 \\
\hline \multirow[t]{2}{*}{ NAEVA } & Targets Detected & 18 & 45 & 20 & 83 \\
\hline & $\mathrm{P}($ det $)$ & 0.750 & 0.556 & 0.588 & 0.597 \\
\hline \multirow[t]{2}{*}{ GTL } & Targets Detected & 18 & 41 & 18 & 77 \\
\hline & $P($ det $)$ & 0.750 & 0.506 & 0.529 & 0.554 \\
\hline \multirow[t]{2}{*}{ Geophex } & Targets Detected & 20 & 46 & 15 & 81 \\
\hline & $\mathrm{P}($ det $)$ & 0.833 & 0.568 & 0.441 & 0.583 \\
\hline \multirow[t]{2}{*}{ NRL } & Targets Detected & 10 & 23 & 8 & 37 \\
\hline & $\mathrm{P}(\mathrm{det})$ & 0.417 & 0.284 & 0.235 & 0.266 \\
\hline \multirow[t]{2}{*}{$\mathrm{NRL}$ without $20 / 40 \mathrm{~mm}$} & Targets Detected & 11 & 33 & 12 & 56 \\
\hline & $P($ det $)$ & 0.458 & 0.407 & 0.353 & 0.403 \\
\hline \multirow[t]{2}{*}{$\begin{array}{l}\text { Parsons EM-61 } \\
\text { EM and Flag }\end{array}$} & Targets Detected & 18 & 50 & 22 & 90 \\
\hline & $P($ det $)$ & 0.750 & 0.617 & 0.647 & 0.647 \\
\hline \multirow[t]{2}{*}{ Parsons EM-61 Digital } & Targets Detected & 18 & 49 & 23 & 90 \\
\hline & $P($ det $)$ & 0.750 & 0.605 & 0.676 & 0.647 \\
\hline \multirow[t]{2}{*}{ Parsons TM-5 EMU } & Targets Detected & 7 & 22 & 4 & 33 \\
\hline & $P($ det $)$ & 0.292 & 0.272 & 0.118 & 0.237 \\
\hline
\end{tabular}

These results indicate that no demonstrator was able to achieve the Kaho'olawe Tier II clearance requirements of $0.85 \mathrm{P}(\mathrm{det})$ with 0.5 -m location accuracy at any of the three demonstration areas. Only when the requirements were relaxed by expanding the allowable position error to $1.0 \mathrm{~m}$ and also deleting the smaller UXO targets did any of the demonstrators meet the P(det) requirements. Even then, acceptable P(det) levels were only obtained in Area A. Area A had considerably lower levels of geologic noise and metallic clutter than the other two areas. 


\begin{tabular}{|c|c|c|c|c|c|}
\hline \multicolumn{6}{|c|}{$\begin{array}{l}\text { Table C2 } \\
\text { P(det) by Area within } 1.0 \mathrm{~m} \text { and without } 20 / 40 \mathrm{~mm}\end{array}$} \\
\hline & & \multicolumn{3}{|c|}{ Within $1.0 \mathrm{~m}$} & \multirow[b]{2}{*}{ Total } \\
\hline & & Area A & Area B & Area C & \\
\hline & $\begin{array}{l}\text { Number of Actual } \\
\text { Targets without } \\
20 / 40 \mathrm{~mm} \\
\end{array}$ & 19 & 55 & 28 & 102 \\
\hline \multirow[t]{2}{*}{ NAEVA } & Targets Detected & 16 & 38 & 19 & 73 \\
\hline & $P($ det $)$ & 0.842 & 0.691 & 0.679 & 0.716 \\
\hline \multirow[t]{2}{*}{ GTL } & Targets Detected & 17 & 31 & 15 & 63 \\
\hline & $\mathrm{P}(\mathrm{det})$ & 0.895 & 0.564 & 0.536 & 0.618 \\
\hline \multirow[t]{2}{*}{ Geophex } & Targets Detected & 17 & 39 & 14 & 70 \\
\hline & $\mathrm{P}$ (det) & 0.895 & 0.709 & 0.500 & 0.686 \\
\hline \multirow[t]{2}{*}{ NRL } & Targets Detected & 9 & 22 & 8 & 37 \\
\hline & $\mathrm{P}(\mathrm{det})$ & 0.474 & 0.400 & 0.286 & 0.363 \\
\hline \multirow[t]{2}{*}{ NRL without $20 / 40 \mathrm{~mm}$} & Targets Detected & 10 & 32 & 11 & 53 \\
\hline & $P$ (det) & 0.526 & 0.582 & 0.393 & 0.520 \\
\hline \multirow[t]{2}{*}{$\begin{array}{l}\text { Parsons EM-61 } \\
\text { EM and Flag }\end{array}$} & Targets Detected & 17 & 42 & 21 & 80 \\
\hline & $\mathrm{P}($ det $)$ & 0.895 & 0.764 & 0.750 & 0.784 \\
\hline \multirow[t]{2}{*}{ Parsons EM-61 Digital } & Targets Detected & 16 & 39 & 22 & 77 \\
\hline & $P($ det $)$ & 0.842 & 0.709 & 0.786 & 0.755 \\
\hline \multirow[t]{2}{*}{ Parsons TM-5 EMU } & Targets Detected & 7 & 19 & 4 & 30 \\
\hline & $\mathrm{P}($ det $)$ & 0.368 & 0.345 & 0.143 & 0.294 \\
\hline
\end{tabular}

Table $\mathrm{C} 3$ shows the number of UXO targets detected within $1.0 \mathrm{~m}$ by each demonstrator in each $30-\times 30-\mathrm{m}$ grid. Shaded entries indicate the highest number of detections for that grid. The last column of the table indicates the number of UXO targets not detected by any of the demonstrators. Tables 4 through 11 show detections by ordnance type for each demonstrator. Sixteen different ordnance types are listed in each table. No UXO targets of the other two types listed in the demonstration plan (BDU 3 practice bomb and 3-in. projectile practice bomb) were emplaced for this demonstration.

\section{Discrimination results}

Tables C12 through 27 illustrate the comparison of the demonstrators' declarations versus the ground-truth emplaced targets. Table C15 summarizes the performance of the demonstrators over the entire demonstration site within the QA Range. In this table, the ordnance items left in the ground represent the items declared by the demonstrator as clutter with high confidence that are actually groundtruth emplaced ordnance plus the number of undetected ordnance items. The variable Correct Discrimination is the total of the correctly identified ordnance items. The False Alarm number is the total of the other detections plus the total of the Clutter minus the number of objects identified as Clutter with High confidence. 


\begin{tabular}{|c|c|c|c|c|c|c|c|c|c|}
\hline \multicolumn{10}{|c|}{$\begin{array}{l}\text { Table C3 } \\
\text { Grid-by-Grid Detections within } 1.0 \text { m }\end{array}$} \\
\hline Grids & $\begin{array}{l}\text { Total } \\
\text { Items } \\
\text { Buried } \\
\end{array}$ & NAEVA & GTL & Geophex & NRL & $\begin{array}{l}\text { Parsons } \\
\text { EM-61 } \\
\text { EM and } \\
\text { Flag } \\
\end{array}$ & $\begin{array}{l}\text { Parsons } \\
\text { EM-61 } \\
\text { Digital } \\
\end{array}$ & $\begin{array}{l}\text { Parsons } \\
\text { TM-5 EMU }\end{array}$ & \begin{tabular}{|l} 
Items \\
Not \\
Found \\
\end{tabular} \\
\hline $1 \mathrm{~A}$ & 6 & 5 & 4 & 5 & 4 & 5 & 6 & 0 & 0 \\
\hline 1B & 5 & 2 & 1 & 2 & 1 & 3 & 3 & 0 & 2 \\
\hline $1 \mathrm{C}$ & 5 & 4 & 2 & 1 & 1 & 4 & 5 & 0 & 0 \\
\hline $1 \mathrm{D}$ & 4 & 4 & 3 & 4 & 0 & 3 & 3 & 0 & 0 \\
\hline $1 \mathrm{E}$ & 7 & 5 & 5 & 6 & 5 & 5 & 5 & 2 & 0 \\
\hline $2 \mathrm{~A}$ & 7 & 5 & 5 & 5 & 0 & 5 & 3 & 2 & 0 \\
\hline $2 B$ & 5 & 3 & 4 & 1 & 2 & 3 & 4 & 2 & 0 \\
\hline $2 \mathrm{C}$ & 6 & 1 & 2 & 1 & 0 & 2 & 2 & 0 & 2 \\
\hline $2 \mathrm{D}$ & 7 & 5 & 6 & 7 & 4 & 5 & 5 & 3 & 0 \\
\hline $2 \mathrm{E}$ & 6 & 4 & 4 & 3 & 1 & 5 & 5 & 2 & 1 \\
\hline $3 B$ & 9 & 9 & 4 & 5 & 2 & 6 & 7 & 1 & 0 \\
\hline $3 C$ & 7 & 4 & 5 & 6 & 2 & 3 & 5 & 2 & 0 \\
\hline $3 \mathrm{D}$ & 6 & 2 & 4 & 3 & 1 & 4 & 3 & 0 & 1 \\
\hline $3 \mathrm{E}$ & 7 & 4 & 4 & 5 & 3 & 4 & 5 & 1 & 2 \\
\hline $4 \mathrm{~B}$ & 6 & 5 & 4 & 5 & 5 & 5 & 5 & 4 & 1 \\
\hline $4 \mathrm{C}$ & 9 & 3 & 5 & 6 & 0 & 5 & 5 & 3 & 1 \\
\hline $4 \mathrm{D}$ & 10 & 7 & 4 & 5 & 4 & 8 & 5 & 5 & 1 \\
\hline $4 \mathrm{E}$ & 10 & 4 & 4 & 3 & 2 & 6 & 4 & 2 & 3 \\
\hline $5 \mathrm{~B}$ & 2 & 1 & 0 & 1 & 1 & 1 & 2 & 0 & 0 \\
\hline $5 C$ & 8 & 3 & 4 & 4 & 1 & 2 & 4 & 1 & 3 \\
\hline $5 \mathrm{D}$ & 3 & 1 & 1 & 2 & 1 & 3 & 2 & 1 & 0 \\
\hline $5 \mathrm{E}$ & 4 & 2 & 2 & 1 & 1 & 3 & 2 & 2 & 0 \\
\hline Totals & 139 & 83 & 77 & 81 & 41 & 90 & 90 & 33 & 17 \\
\hline
\end{tabular}

\begin{tabular}{|c|c|c|c|c|c|c|c|c|}
\hline \multicolumn{9}{|c|}{\begin{tabular}{|l|} 
Table C4 \\
UXO Found - NAEVA
\end{tabular}} \\
\hline \multirow[b]{3}{*}{ Description } & \multicolumn{8}{|c|}{ Area } \\
\hline & \multicolumn{4}{|c|}{ Actual Targets } & \multicolumn{4}{|c|}{ Within $1.0 \mathrm{~m}$} \\
\hline & A & B & C & Total & A & $\mathrm{B}$ & C & Total \\
\hline 2.25-in. & 0 & 3 & 0 & 3 & 0 & 3 & 0 & 3 \\
\hline 2.75-in. & 4 & 5 & 4 & 13 & 4 & 1 & 2 & 7 \\
\hline 5-in. & 2 & 4 & 2 & 8 & 2 & 3 & 2 & 7 \\
\hline 20-mm & 4 & 12 & 1 & 17 & 1 & 0 & 0 & 1 \\
\hline 40-mm & 1 & 14 & 5 & 20 & 1 & 7 & 1 & 9 \\
\hline 60-mm & 2 & 11 & 3 & 16 & 2 & 7 & 1 & 10 \\
\hline $81-\mathrm{mm}$ & 1 & 8 & 3 & 12 & 1 & 7 & 1 & 9 \\
\hline 105-mm & 1 & 3 & 1 & 5 & 1 & 2 & 1 & 4 \\
\hline BDU-33 & 3 & 4 & 3 & 10 & 3 & 3 & 2 & 8 \\
\hline Mk-3 & 1 & 4 & 3 & 8 & 1 & 4 & 3 & 8 \\
\hline Mk-81 & 1 & 2 & 1 & 4 & 1 & 0 & 1 & 2 \\
\hline Mk-82 & 1 & 4 & 7 & 12 & 0 & 3 & 5 & 8 \\
\hline Mk-83 & 0 & 1 & 0 & 1 & 0 & 0 & 0 & 0 \\
\hline \begin{tabular}{|l|} 
Mk-106 \\
\end{tabular} & 0 & 4 & 0 & 4 & 0 & 4 & 0 & 4 \\
\hline LAAW & 2 & 1 & 0 & 3 & 1 & 0 & 0 & 1 \\
\hline SMAW & 1 & 1 & 1 & 3 & 0 & 1 & 1 & 2 \\
\hline Total & 24 & 81 & 34 & 139 & 18 & 45 & 20 & 83 \\
\hline
\end{tabular}




\begin{tabular}{|c|c|c|c|c|c|c|c|c|}
\hline \multirow[b]{4}{*}{ Description } & d - & & & & & & & \\
\hline & \multicolumn{8}{|c|}{ Area } \\
\hline & \multicolumn{4}{|c|}{ Actual Targets } & \multicolumn{4}{|c|}{ Within $1.0 \mathrm{~m}$} \\
\hline & $\mathbf{A}$ & B & C & Total & A & B & C & Total \\
\hline 2.25-in. & 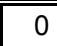 & 3 & 0 & 3 & 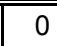 & 2 & 0 & 2 \\
\hline 2.75-in. & 4 & 5 & 4 & 13 & 4 & 0 & 1 & 5 \\
\hline 5-in. & 2 & 4 & 2 & 8 & 2 & 3 & 2 & 7 \\
\hline $20-\mathrm{mm}$ & 4 & 12 & 1 & 17 & 1 & 3 & 1 & 5 \\
\hline 40-mm & 1 & 14 & 5 & 20 & 0 & 9 & 2 & 11 \\
\hline 60-mm & 2 & 11 & 3 & 16 & 2 & 5 & 3 & 10 \\
\hline 81-mm & 1 & 8 & 3 & 12 & 1 & 7 & 1 & 9 \\
\hline $105 \mathrm{~mm}$ & 1 & 3 & 1 & 5 & 1 & 2 & 1 & 4 \\
\hline BDU-33 & 3 & 4 & 3 & 10 & 3 & 2 & 2 & 7 \\
\hline Mk-3 & 1 & 4 & 3 & 8 & 1 & 2 & 1 & 4 \\
\hline Mk-81 & 1 & 2 & 1 & 4 & 1 & 0 & 0 & 1 \\
\hline Mk-82 & 1 & 4 & 7 & 12 & 1 & 2 & 4 & 7 \\
\hline Mk-83 & & 1 & & 1 & 0 & 0 & 0 & 0 \\
\hline Mk-106 & 0 & 4 & 0 & 4 & 0 & 4 & 0 & 4 \\
\hline LAAW & 2 & 1 & 0 & 3 & 1 & 0 & 0 & 1 \\
\hline SMAW & 1 & 1 & 1 & 3 & 0 & 0 & 0 & 0 \\
\hline Total & 24 & 81 & 34 & 139 & 18 & 41 & 18 & 77 \\
\hline
\end{tabular}

\begin{tabular}{|c|c|c|c|c|c|c|c|c|}
\hline $\begin{array}{l}\text { Table C6 } \\
\text { UXO Fou }\end{array}$ & d - & pr & 4 & ex & 10u & 40 & & \\
\hline & & & & & rea & & & \\
\hline & & & Targ & & & & 1.0 & \\
\hline Description & A & B & C & Total & A & B & C & Total \\
\hline 2.25-in. & 0 & 3 & 0 & 3 & 0 & 3 & 0 & 3 \\
\hline 2.75-in. & 4 & 5 & 4 & 13 & 4 & 2 & 1 & 7 \\
\hline 5-in. & 2 & 4 & 2 & 8 & 1 & 4 & 2 & 7 \\
\hline 20-mm & 4 & 12 & 1 & 17 & 2 & 1 & 0 & 3 \\
\hline 40-mm & 1 & 14 & 5 & 20 & 1 & 6 & 1 & 8 \\
\hline 60-mm & 2 & 11 & 3 & 16 & 2 & 5 & 2 & 9 \\
\hline 81-mm & 1 & 8 & 3 & 12 & 1 & 8 & 1 & 10 \\
\hline 105-mm & 1 & 3 & 1 & 5 & 1 & 3 & 0 & 4 \\
\hline BDU-33 & 3 & 4 & 3 & 10 & 3 & 3 & 2 & 8 \\
\hline Mk-3 & 1 & 4 & 3 & 8 & 1 & 4 & 2 & 7 \\
\hline Mk-81 & 1 & 2 & 1 & 4 & 1 & 1 & 1 & 3 \\
\hline Mk-82 & 1 & 4 & 7 & 12 & 1 & 2 & 3 & 6 \\
\hline Mk-83 & & 1 & & 1 & 0 & 0 & 0 & 0 \\
\hline Mk-106 & 0 & 4 & 0 & 4 & 0 & 4 & 0 & 4 \\
\hline LAAW & 2 & 1 & 0 & 3 & 1 & 0 & 0 & 1 \\
\hline SMAW & 1 & 1 & 1 & 3 & 1 & 0 & 0 & 1 \\
\hline Total & 24 & 81 & 34 & 139 & 20 & 46 & 15 & 81 \\
\hline
\end{tabular}




\begin{tabular}{|c|c|c|c|c|c|c|c|c|}
\hline \multirow[b]{4}{*}{ Description } & d - & & & & & & & \\
\hline & \multicolumn{8}{|c|}{ Area } \\
\hline & \multicolumn{4}{|c|}{ Actual Targets } & \multicolumn{4}{|c|}{ Within $1.0 \mathrm{~m}$} \\
\hline & $\mathbf{A}$ & B & C & Total & A & B & C & Total \\
\hline 2.25-in. & 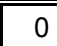 & 3 & 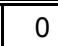 & 3 & 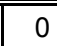 & 2 & 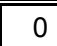 & 2 \\
\hline 2.75-in. & 4 & 5 & 4 & 13 & 1 & 1 & 1 & 3 \\
\hline 5-in. & 2 & 4 & 2 & 8 & 1 & 2 & 0 & 3 \\
\hline 20-mm & 4 & 12 & 1 & 17 & 1 & 0 & 0 & 1 \\
\hline 40-mm & 1 & 14 & 5 & 20 & 0 & 1 & 0 & 1 \\
\hline 60-mm & 2 & 11 & 3 & 16 & 1 & 2 & 1 & 4 \\
\hline $81-\mathrm{mm}$ & 1 & 8 & 3 & 12 & 0 & 4 & 0 & 4 \\
\hline $105-\mathrm{mm}$ & 1 & 3 & 1 & 5 & 0 & 2 & 0 & 2 \\
\hline BDU-33 & 3 & 4 & 3 & 10 & 3 & 3 & 1 & 7 \\
\hline Mk-3 & 1 & 4 & 3 & 8 & 1 & 2 & 1 & 4 \\
\hline Mk-81 & 1 & 2 & 1 & 4 & 1 & 0 & 1 & 2 \\
\hline Mk-82 & 1 & 4 & 7 & 12 & 0 & 2 & 3 & 5 \\
\hline Mk-83 & & 1 & & 1 & 0 & 0 & 0 & 0 \\
\hline Mk-106 & 0 & 4 & 0 & 4 & 0 & 2 & 0 & 2 \\
\hline LAAW & 2 & 1 & 0 & 3 & 1 & 0 & 0 & 1 \\
\hline SMAW & 1 & 1 & 1 & 3 & 0 & 0 & 0 & 0 \\
\hline Total & 24 & 81 & 34 & 139 & 10 & 23 & 8 & 41 \\
\hline
\end{tabular}

\begin{tabular}{|c|c|c|c|c|c|c|c|c|}
\hline \multicolumn{9}{|c|}{$\begin{array}{l}\text { Table C8 } \\
\text { UXO Found - NRL without } 20 / 40 \mathrm{~mm}\end{array}$} \\
\hline \multirow[b]{3}{*}{ Description } & \multicolumn{8}{|c|}{ Area } \\
\hline & \multicolumn{4}{|c|}{ Actual Targets } & \multicolumn{4}{|c|}{ Within $1.0 \mathrm{~m}$} \\
\hline & $\mathbf{A}$ & B & C & Total & A & B & C & Total \\
\hline 2.25-in. & 0 & 3 & 0 & 3 & 0 & 3 & 0 & 3 \\
\hline 2.75-in. & 4 & 5 & 4 & 13 & 1 & 1 & 1 & 3 \\
\hline 5-in. & 2 & 4 & 2 & 8 & 1 & 3 & 0 & 4 \\
\hline 20-mm & 4 & 12 & 1 & 17 & 1 & 0 & 0 & 1 \\
\hline 40-mm & 1 & 14 & 5 & 20 & 0 & 1 & 1 & 2 \\
\hline 60-mm & 2 & 11 & 3 & 16 & 2 & 4 & 2 & 8 \\
\hline $81-\mathrm{mm}$ & 1 & 8 & 3 & 12 & 0 & 5 & 1 & 6 \\
\hline 105-mm & 1 & 3 & 1 & 5 & 0 & 3 & 0 & 3 \\
\hline BDU-33 & 3 & 4 & 3 & 10 & 3 & 4 & 1 & 8 \\
\hline Mk-3 & 1 & 4 & 3 & 8 & 1 & 4 & 1 & 6 \\
\hline Mk-81 & 1 & 2 & 1 & 4 & 1 & 0 & 1 & 2 \\
\hline Mk-82 & 1 & 4 & 7 & 12 & 0 & 2 & 3 & 5 \\
\hline Mk-83 & & 1 & & 1 & 0 & 0 & 0 & 0 \\
\hline Mk-106 & 0 & 4 & 0 & 4 & 0 & 3 & 0 & 3 \\
\hline LAAW & 2 & 1 & 0 & 3 & 1 & 0 & 0 & 1 \\
\hline SMAW & 1 & 1 & 1 & 3 & 0 & 0 & 1 & 1 \\
\hline Total & 24 & 81 & 34 & 139 & 11 & 33 & 12 & 56 \\
\hline
\end{tabular}




\begin{tabular}{|c|c|c|c|c|c|c|c|c|}
\hline \multicolumn{9}{|c|}{$\begin{array}{l}\text { Table C9 } \\
\text { UXO Found - Parsons EM-61 EM \& Flag }\end{array}$} \\
\hline \multirow[b]{3}{*}{ Description } & \multicolumn{8}{|c|}{ Area } \\
\hline & \multicolumn{4}{|c|}{ Actual Targets } & \multicolumn{4}{|c|}{ Within $1.0 \mathrm{~m}$} \\
\hline & $\mathbf{A}$ & B & C & Total & A & B & C & Total \\
\hline 2.25-in. & 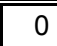 & 3 & 0 & 3 & 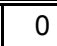 & 3 & 0 & 3 \\
\hline 2.75-in. & 4 & 5 & 4 & 13 & 4 & 3 & 1 & 8 \\
\hline 5-in. & 2 & 4 & 2 & 8 & 2 & 4 & 2 & 8 \\
\hline $20-\mathrm{mm}$ & 4 & 12 & 1 & 17 & 0 & 1 & 0 & 1 \\
\hline 40-mm & 1 & \begin{tabular}{|l|}
14 \\
\end{tabular} & 5 & 20 & 1 & 7 & 1 & 9 \\
\hline 60-mm & 2 & 11 & 3 & 16 & 1 & 6 & 3 & 10 \\
\hline 81-mm & 1 & 8 & 3 & 12 & 1 & 7 & 2 & 10 \\
\hline $105-\mathrm{mm}$ & 1 & 3 & 1 & 5 & 1 & 2 & 1 & 4 \\
\hline BDU-33 & 3 & 4 & 3 & 10 & 3 & 4 & 2 & 9 \\
\hline Mk-3 & 1 & 4 & 3 & 8 & 1 & 4 & 3 & 8 \\
\hline Mk-81 & 1 & 2 & 1 & 4 & 1 & 2 & 1 & 4 \\
\hline Mk-82 & 1 & 4 & 7 & 12 & 1 & 3 & 5 & 9 \\
\hline Mk-83 & & 1 & & 1 & 0 & 0 & 0 & 0 \\
\hline Mk-106 & 0 & 4 & 0 & 4 & 0 & 4 & 0 & 4 \\
\hline LAAW & 2 & 1 & 0 & 3 & 2 & 0 & 0 & 2 \\
\hline SMAW & 1 & 1 & 1 & 3 & 0 & 0 & 1 & 1 \\
\hline Total & 24 & 81 & 34 & 139 & 18 & 50 & 22 & 90 \\
\hline
\end{tabular}

\begin{tabular}{|c|c|c|c|c|c|c|c|c|}
\hline \multicolumn{9}{|c|}{$\begin{array}{l}\text { Table C10 } \\
\text { UXO Found - Parsons EM-61 Digital }\end{array}$} \\
\hline \multirow[b]{3}{*}{ Description } & \multicolumn{8}{|c|}{ Area } \\
\hline & \multicolumn{4}{|c|}{ Actual Targets } & \multicolumn{4}{|c|}{ Within $1.0 \mathrm{~m}$} \\
\hline & A & $B$ & C & Total & A & B & C & Total \\
\hline 2.25-in. & 0 & 3 & 0 & 3 & 0 & 2 & 0 & 2 \\
\hline 2.75-in. & 4 & 5 & 4 & 13 & 4 & 2 & 2 & 8 \\
\hline 5-in. & 2 & 4 & 2 & 8 & 2 & 3 & 1 & 6 \\
\hline 20-mm & 4 & 12 & 1 & 17 & 1 & 1 & 0 & 2 \\
\hline 40-mm & 1 & 14 & 5 & 20 & 1 & 9 & 1 & 11 \\
\hline 60-mm & 2 & 11 & 3 & 16 & 2 & 8 & 3 & 13 \\
\hline $81-\mathrm{mm}$ & 1 & 8 & 3 & 12 & 1 & 8 & 2 & 11 \\
\hline 105-mm & 1 & 3 & 1 & 5 & 1 & 3 & 1 & 5 \\
\hline BDU-33 & 3 & 4 & 3 & 10 & 2 & 3 & 3 & 8 \\
\hline Mk-3 & 1 & 4 & 3 & 8 & 1 & 2 & 3 & 6 \\
\hline Mk-81 & 1 & 2 & 1 & 4 & 1 & 1 & 1 & 3 \\
\hline Mk-82 & 1 & 4 & 7 & 12 & 0 & 2 & 5 & 7 \\
\hline Mk-83 & 0 & 1 & 0 & 1 & 0 & 0 & 0 & 0 \\
\hline Mk-106 & 0 & 4 & 0 & 4 & 0 & 4 & 0 & 4 \\
\hline LAAW & 2 & 1 & 0 & 3 & 2 & 1 & 0 & 3 \\
\hline SMAW & 1 & 1 & 1 & 3 & 0 & 0 & 1 & 1 \\
\hline Total & 24 & 81 & 34 & 139 & 18 & 49 & 23 & 90 \\
\hline
\end{tabular}




\begin{tabular}{|c|c|c|c|c|c|c|c|c|}
\hline \multicolumn{9}{|c|}{$\begin{array}{l}\text { Table C11 } \\
\text { UXO Found - Parsons EM-61 TM-5 EMU }\end{array}$} \\
\hline \multirow[b]{3}{*}{ Description } & \multicolumn{8}{|c|}{ Area } \\
\hline & \multicolumn{4}{|c|}{ Actual Targets } & \multicolumn{4}{|c|}{ Within $1.0 \mathrm{~m}$} \\
\hline & A & B & C & Total & A & $\mathrm{B}$ & C & Total \\
\hline 2.25-in. & 0 & 3 & 0 & 3 & 0 & 1 & 0 & 1 \\
\hline 2.75-in. & 4 & 5 & 4 & 13 & 0 & 3 & 0 & 3 \\
\hline 5-in. & 2 & 4 & 2 & 8 & 0 & 1 & 0 & 1 \\
\hline 20-mm & 4 & 12 & 1 & 17 & 0 & 0 & 0 & 0 \\
\hline 40-mm & 1 & 14 & 5 & 20 & 0 & 3 & 0 & 3 \\
\hline 60-mm & 2 & 11 & 3 & 16 & 1 & 4 & 1 & 6 \\
\hline 81-mm & 1 & 8 & 3 & 12 & 1 & 1 & 1 & 3 \\
\hline 105-mm & 1 & 3 & 1 & 5 & 1 & 1 & 0 & 2 \\
\hline BDU-33 & 3 & 4 & 3 & 10 & 0 & 0 & 0 & 0 \\
\hline Mk-3 & 1 & 4 & 3 & 8 & 3 & 2 & 0 & 5 \\
\hline Mk-81 & 1 & 2 & 1 & 4 & 0 & 1 & 0 & 1 \\
\hline Mk-82 & 1 & 4 & 7 & 12 & 0 & 1 & 0 & 1 \\
\hline Mk-83 & 0 & 1 & 0 & 1 & 0 & 0 & 0 & 0 \\
\hline Mk-106 & 0 & 4 & 0 & 4 & 1 & 1 & 0 & 2 \\
\hline LAAW & 2 & 1 & 0 & 3 & 0 & 3 & 2 & 5 \\
\hline SMAW & 1 & 1 & 1 & 3 & 0 & 0 & 0 & 0 \\
\hline Total & 24 & 81 & 34 & 139 & 7 & 22 & 4 & 33 \\
\hline
\end{tabular}

Table 13 through 27 show more detailed breakdowns of the results by area for each demonstrator. The left most column of each of these tables represents the emplaced targets. These items are matched with the demonstrators' dig list. When the demonstrator reported the object as ordnance and the emplaced target was a groundtruth ordnance match then it is considered a correct discrimination. The confidence of the demonstrators' declaration is used to determine which items from his dig list are to be excavated. In lieu of a demonstrators' defined "stop dig point," only objects that are declared as clutter with high confidence would be left in the ground.

For example, NAEVA (Under $1.0 \mathrm{~m}$ ) discrimination performance is illustrated in Table C13. NAEVA reported 295 items as Ordnance. Of that number, 51 actually matched the ground-truth ordnance items emplaced in the ground, 39 matched the ground-truth emplaced fragments, and 205 detected something other than the targets emplaced in the ground. NAEVA reported 630 items as clutter. Only 37 actually matched the ground-truth fragments emplaced in the ground, 32 matched the ground-truth emplaced ordnance items, and 561 detected something other than the targets emplaced in the ground. NAEVA reported $250(240+4+6)$ with high confidence of the 630 clutter items, therefore, 40 percent of the identified clutter were designated as "do not dig." There were six ordnance items that would have been left in the ground as a result of being classified as high confidence clutter. Also, from Table C12, there were 62 undetected ordnance items. NAEVA reported a total of 766 items which did not match any emplaced ordnance, and 76 items that matched emplaced fragments. 


\begin{tabular}{|c|c|c|c|c|c|}
\hline \multicolumn{6}{|c|}{$\begin{array}{l}\text { Table C12 } \\
\text { Summary of Discrimination Performance }\end{array}$} \\
\hline & \multicolumn{2}{|c|}{$\begin{array}{c}\text { Ordnance Left in the } \\
\text { Ground }\end{array}$} & \multicolumn{2}{|c|}{ Correct Discrimination } & \begin{tabular}{|l|} 
False \\
Alarm \\
Number
\end{tabular} \\
\hline & \multicolumn{2}{|c|}{ Within $1.0 \mathrm{~m}$} & \multicolumn{2}{|c|}{ Within $1.0 \mathrm{~m}$} & \multirow{2}{*}{$\begin{array}{l}\text { Within } \\
1.0 \mathrm{~m}\end{array}$} \\
\hline & Num & Percent & Num & Percent & \\
\hline Naeva & 262 & 44.60 & 77 & 64.03 & 842 \\
\hline GTL & 62 & 45.32 & 77 & 55.40 & 1,249 \\
\hline Geophex & 58 & 41.73 & 81 & 58.27 & 750 \\
\hline $\begin{array}{l}\text { Geophex without } \\
20 / 40 \mathrm{~mm}\end{array}$ & 58 & 41.73 & 81 & 58.27 & 750 \\
\hline NRL & 98 & 70.50 & 41 & 29.50 & 333 \\
\hline \begin{tabular}{|l|} 
NRL without \\
$20 / 40 \mathrm{~mm}$
\end{tabular} & 83 & 59.71 & 56 & 40.29 & 584 \\
\hline $\begin{array}{l}\text { Parson (EM-61) } \\
\text { EM \& Flag }\end{array}$ & 49 & 35.25 & 90 & 64.75 & 854 \\
\hline Parson EM-61 Digital & 49 & 35.25 & 90 & 64.75 & 1,530 \\
\hline Parson TM-5 EMU & 106 & 76.26 & 33 & 23.74 & 167 \\
\hline
\end{tabular}

\begin{tabular}{|c|c|c|c|c|c|c|c|}
\hline \multicolumn{8}{|c|}{$\begin{array}{l}\text { Table C13 } \\
\text { Breakdown of Discrimination - QA Range and Area A - NAEVA }\end{array}$} \\
\hline \multirow[b]{3}{*}{$\begin{array}{l}\text { Ground- } \\
\text { truth }\end{array}$} & \multicolumn{7}{|c|}{ Demonstrator Classification } \\
\hline & \multirow[b]{2}{*}{ Confidence } & \multicolumn{3}{|c|}{ QA Range } & \multicolumn{3}{|c|}{ Area A } \\
\hline & & $\begin{array}{l}\text { Total } \\
\text { Anomalies }\end{array}$ & $\begin{array}{l}\text { Declared } \\
\text { Ordnance }\end{array}$ & $\begin{array}{l}\text { Declared } \\
\text { Clutter }\end{array}$ & $\begin{array}{l}\text { Total } \\
\text { Anomalies }\end{array}$ & $\begin{array}{l}\text { Declared } \\
\text { Ordnance }\end{array}$ & $\begin{array}{l}\text { Declared } \\
\text { Clutter }\end{array}$ \\
\hline \multirow{3}{*}{$\begin{array}{l}\text { Non- } \\
\text { emplaced }\end{array}$} & High & 366 & 126 & 240 & 168 & 28 & 140 \\
\hline & Low & 400 & 79 & 321 & 94 & 22 & 72 \\
\hline & Total & 766 & 205 & 561 & 262 & 50 & 212 \\
\hline \multirow[t]{3}{*}{ Clutter } & High & 33 & 29 & 4 & 10 & 7 & 3 \\
\hline & Low & 43 & 10 & 33 & 9 & 2 & 7 \\
\hline & Total & 76 & 39 & 37 & 19 & 9 & 10 \\
\hline \multirow[t]{3}{*}{ Ordnance } & High & 43 & 37 & 6 & 10 & 8 & 2 \\
\hline & Low & 40 & 14 & 26 & 8 & 2 & 6 \\
\hline & Total & 83 & 51 & 32 & 18 & 10 & 8 \\
\hline Total & & 925 & 295 & 630 & 299 & 69 & 230 \\
\hline
\end{tabular}




\begin{tabular}{|c|c|c|c|c|c|c|c|}
\hline \multicolumn{8}{|c|}{$\begin{array}{l}\text { Table C14 } \\
\text { Breakdown of Discrimination - Area B and Area C - NAEVA }\end{array}$} \\
\hline \multirow[b]{3}{*}{$\begin{array}{l}\text { Ground- } \\
\text { truth }\end{array}$} & \multicolumn{7}{|c|}{ Demonstrator Classification } \\
\hline & \multirow[b]{2}{*}{ Confidence } & \multicolumn{3}{|c|}{ Area B } & \multicolumn{3}{|l|}{ Area C } \\
\hline & & $\begin{array}{l}\text { Total } \\
\text { Anomalies }\end{array}$ & $\begin{array}{l}\text { Declared } \\
\text { Ordnance }\end{array}$ & $\begin{array}{l}\text { Declared } \\
\text { Clutter }\end{array}$ & $\begin{array}{l}\text { Total } \\
\text { Anomalie } \\
\text { s }\end{array}$ & $\begin{array}{l}\text { Declared } \\
\text { Ordnance }\end{array}$ & $\begin{array}{l}\text { Declared } \\
\text { Clutter }\end{array}$ \\
\hline \multirow{3}{*}{$\begin{array}{l}\text { Non- } \\
\text { emplaced }\end{array}$} & High & 121 & 57 & 64 & 77 & 41 & 36 \\
\hline & Low & 173 & 31 & 142 & 133 & 26 & 107 \\
\hline & Total & 294 & 88 & 206 & 210 & 67 & 143 \\
\hline \multirow[t]{3}{*}{ Clutter } & High & 15 & 14 & 1 & 8 & 8 & 0 \\
\hline & Low & 18 & 3 & 15 & 16 & 5 & 11 \\
\hline & Total & 33 & 17 & 16 & 24 & 13 & 11 \\
\hline \multirow[t]{3}{*}{ Ordnance } & High & 22 & 19 & 3 & 11 & 10 & 1 \\
\hline & Low & 23 & 8 & 15 & 9 & 4 & 5 \\
\hline & Total & 45 & 27 & 18 & 20 & 14 & 6 \\
\hline Total & & 372 & 132 & 240 & 254 & 94 & \begin{tabular}{|l|}
160 \\
\end{tabular} \\
\hline
\end{tabular}

\begin{tabular}{|c|c|c|c|c|c|}
\hline $\begin{array}{l}\text { Table C } \\
\text { Breakd }\end{array}$ & vn of Disc & minatior & QA Range & GTL & \\
\hline & & & ionstrator $\mathrm{Cl}$ & ication & \\
\hline & & Total & Area A & Area B & Area C \\
\hline $\begin{array}{l}\text { Ground- } \\
\text { truth }\end{array}$ & Confidence & $\begin{array}{l}\text { Declared } \\
\text { Ordnance }\end{array}$ & $\begin{array}{l}\text { Declared } \\
\text { Ordnance }\end{array}$ & $\begin{array}{l}\text { Declared } \\
\text { Ordnance }\end{array}$ & $\begin{array}{l}\text { Declared } \\
\text { Ordnance }\end{array}$ \\
\hline Non- & High & 649 & 90 & 315 & 244 \\
\hline emplaced & Low & 293 & 65 & 158 & 70 \\
\hline & Medium & 225 & 52 & 117 & 56 \\
\hline & Total & 1,167 & 207 & 590 & 370 \\
\hline Clutter & High & 58 & 15 & 28 & 15 \\
\hline & Low & 15 & 2 & 9 & 4 \\
\hline & Medium & 9 & 4 & 4 & 1 \\
\hline & Total & 82 & 21 & 41 & 20 \\
\hline Ordnance & High & 56 & 12 & 29 & 15 \\
\hline & Low & 10 & 2 & 5 & 3 \\
\hline & Medium & 11 & 4 & 7 & 0 \\
\hline & Total & 77 & 18 & 41 & 18 \\
\hline Total & & 1,326 & 246 & 672 & 408 \\
\hline
\end{tabular}




\begin{tabular}{|c|c|c|c|c|c|c|c|}
\hline \multicolumn{8}{|c|}{$\begin{array}{l}\text { Table C16 } \\
\text { Breakdown of Discrimination - QA Range and Area A - Geophex \& } \\
\text { Geophex without } 20 / 40 \mathrm{~mm}\end{array}$} \\
\hline \multirow[b]{3}{*}{$\begin{array}{l}\text { Ground- } \\
\text { truth }\end{array}$} & \multicolumn{7}{|c|}{ Demonstrator Classification } \\
\hline & \multirow[b]{2}{*}{ Confidence } & \multicolumn{3}{|c|}{ QA Range } & \multicolumn{3}{|c|}{ Area A } \\
\hline & & \begin{tabular}{|l|} 
Total \\
Anomalies \\
\end{tabular} & \begin{tabular}{|l|} 
Declared \\
Ordnance
\end{tabular} & \begin{tabular}{|l|} 
Declared \\
Clutter
\end{tabular} & \begin{tabular}{|l|} 
Total \\
Anomalies
\end{tabular} & \begin{tabular}{|l|} 
Declared \\
Ordnance \\
\end{tabular} & \begin{tabular}{|l|} 
Declared \\
Clutter
\end{tabular} \\
\hline \multirow{4}{*}{$\begin{array}{l}\text { Non- } \\
\text { emplaced }\end{array}$} & High & 93 & 89 & 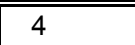 & 15 & 15 & 0 \\
\hline & Low & 276 & 236 & 40 & 51 & 46 & 5 \\
\hline & Medium & 296 & 263 & 33 & 42 & 39 & 3 \\
\hline & Total & 665 & 588 & 77 & 108 & 100 & 8 \\
\hline \multirow{4}{*}{ Clutter } & High & 26 & 26 & 0 & 10 & 10 & 0 \\
\hline & Low & 22 & 18 & 4 & 3 & 2 & 1 \\
\hline & Medium & 41 & 37 & 4 & 9 & 9 & 0 \\
\hline & Total & 89 & 81 & 8 & 22 & 21 & 1 \\
\hline \multirow{4}{*}{ Ordnance } & High & 25 & 25 & 0 & 6 & 6 & 0 \\
\hline & Low & 22 & 21 & 1 & 5 & 5 & 0 \\
\hline & Medium & 34 & 33 & 1 & 9 & 8 & 1 \\
\hline & Total & 81 & 79 & 2 & 20 & 19 & 1 \\
\hline Total & & 835 & 748 & 87 & 150 & 140 & 10 \\
\hline
\end{tabular}

\begin{tabular}{|c|c|c|c|c|c|c|c|}
\hline \multicolumn{8}{|c|}{$\begin{array}{l}\text { Table C17 } \\
\text { Breakdown of Discrimination - Area B and Area C - Geophex \& } \\
\text { Geophex without } 20 / 40 \mathrm{~mm}\end{array}$} \\
\hline \multirow[b]{3}{*}{$\begin{array}{l}\text { Ground- } \\
\text { truth }\end{array}$} & \multicolumn{7}{|c|}{ Demonstrator Classification } \\
\hline & \multirow[b]{2}{*}{ Confidence } & \multicolumn{3}{|c|}{ Area B } & \multicolumn{3}{|c|}{ Area C } \\
\hline & & \begin{tabular}{|l|} 
Total \\
Anomalies
\end{tabular} & \begin{tabular}{|l|} 
Declared \\
Ordnance
\end{tabular} & $\begin{array}{l}\text { Declared } \\
\text { Clutter }\end{array}$ & \begin{tabular}{|l|} 
Total \\
Anomalies
\end{tabular} & $\begin{array}{l}\text { Declared } \\
\text { Ordnance }\end{array}$ & \begin{tabular}{|l} 
Declared \\
Clutter
\end{tabular} \\
\hline \multirow{4}{*}{\begin{tabular}{|l|} 
Non- \\
emplaced
\end{tabular}} & High & 60 & 57 & 3 & 18 & 17 & 1 \\
\hline & Low & 166 & 147 & 19 & 59 & 43 & 16 \\
\hline & Medium & 189 & 166 & 23 & 65 & 58 & 7 \\
\hline & Total & 415 & 370 & 45 & 142 & 118 & 24 \\
\hline \multirow[t]{4}{*}{ Clutter } & High & 12 & 12 & 0 & 4 & 4 & 0 \\
\hline & Low & 13 & 11 & 2 & 6 & 5 & 1 \\
\hline & Medium & 21 & 17 & 4 & 11 & 11 & 0 \\
\hline & Total & 46 & 40 & 6 & 21 & 20 & 1 \\
\hline \multirow[t]{4}{*}{ Ordnances } & High & 14 & 14 & 0 & 5 & 5 & 0 \\
\hline & Low & 13 & 13 & 0 & 4 & 3 & 1 \\
\hline & Medium & 19 & 19 & 0 & 6 & 6 & 0 \\
\hline & Total & 46 & 46 & 0 & 15 & 14 & 1 \\
\hline Total & & 507 & 456 & 51 & 178 & 152 & 26 \\
\hline
\end{tabular}




\begin{tabular}{|c|c|c|c|c|c|c|c|}
\hline \multicolumn{8}{|c|}{$\begin{array}{l}\text { Table C18 } \\
\text { Breakdown of Discrimination - QA Range and Area A - NRL }\end{array}$} \\
\hline \multirow[b]{3}{*}{$\begin{array}{l}\text { Ground- } \\
\text { truth }\end{array}$} & \multicolumn{7}{|c|}{ Demonstrator Classification } \\
\hline & \multirow[b]{2}{*}{ Confidence } & \multicolumn{3}{|c|}{ QA Range } & \multicolumn{3}{|c|}{ Area A } \\
\hline & & \begin{tabular}{|l} 
Total \\
Anomalies
\end{tabular} & \begin{tabular}{|l|} 
Declared \\
Ordnance
\end{tabular} & $\begin{array}{l}\text { Declared } \\
\text { Clutter }\end{array}$ & $\begin{array}{l}\text { Total } \\
\text { Anomalies }\end{array}$ & $\begin{array}{l}\text { Declared } \\
\text { Ordnance }\end{array}$ & $\begin{array}{l}\text { Declared } \\
\text { Clutter }\end{array}$ \\
\hline \multirow{4}{*}{$\begin{array}{l}\text { Non- } \\
\text { emplaced }\end{array}$} & High & 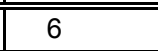 & 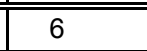 & 0 & 0 & 0 & 0 \\
\hline & Low & 215 & 87 & 128 & 78 & 27 & 51 \\
\hline & Medium & 71 & 55 & 16 & 32 & 23 & 9 \\
\hline & Total & 292 & 148 & 144 & 110 & 50 & 60 \\
\hline \multirow[t]{4}{*}{ Clutter } & High & 11 & 11 & 0 & 0 & 0 & 0 \\
\hline & Low & 17 & 14 & 3 & 5 & 5 & 0 \\
\hline & Medium & 12 & 12 & 0 & 6 & 6 & 0 \\
\hline & Total & 40 & 37 & 3 & 11 & 11 & 0 \\
\hline \multirow[t]{4}{*}{ Ordnance } & High & 6 & 6 & 0 & 0 & 0 & 0 \\
\hline & Low & 21 & 14 & 7 & 4 & 3 & 1 \\
\hline & Medium & 14 & 13 & 1 & 6 & 6 & 0 \\
\hline & Total & 41 & 33 & 8 & 10 & 9 & 1 \\
\hline Total & & 373 & 218 & 155 & 131 & 70 & 61 \\
\hline
\end{tabular}

\begin{tabular}{|c|c|c|c|c|c|c|c|}
\hline \multicolumn{8}{|c|}{$\begin{array}{l}\text { Table C19 } \\
\text { Breakdown of Discrimination - Area B and Area C - NRL }\end{array}$} \\
\hline \multirow[b]{3}{*}{$\begin{array}{l}\text { Ground- } \\
\text { truth }\end{array}$} & \multicolumn{7}{|c|}{ "Demonstrator Classification } \\
\hline & \multirow[b]{2}{*}{ Confidence } & \multicolumn{3}{|c|}{ Area B } & \multicolumn{3}{|c|}{ Area C } \\
\hline & & \begin{tabular}{|l|} 
Total \\
Anomalies \\
\end{tabular} & \begin{tabular}{|l|} 
Declared \\
Ordnance \\
\end{tabular} & \begin{tabular}{|l|} 
Declared \\
Clutter
\end{tabular} & \begin{tabular}{|l|} 
Total \\
Anomalies \\
\end{tabular} & $\begin{array}{l}\text { Declared } \\
\text { Ordnance }\end{array}$ & \begin{tabular}{|l|} 
Declared \\
Clutter \\
\end{tabular} \\
\hline \multirow{4}{*}{$\begin{array}{l}\text { Non- } \\
\text { emplaced }\end{array}$} & High & 2 & 2 & 0 & 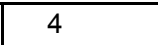 & 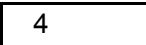 & 0 \\
\hline & Low & 75 & 36 & 39 & 62 & 24 & 38 \\
\hline & Medium & 16 & 12 & 4 & 23 & 20 & 3 \\
\hline & Total & 93 & 50 & 43 & 89 & 48 & 41 \\
\hline \multirow[t]{4}{*}{ Clutter } & High & 7 & 7 & 0 & 4 & 4 & 0 \\
\hline & Low & 8 & 5 & 3 & 4 & 4 & 0 \\
\hline & Medium & 4 & 4 & 0 & 2 & 2 & 0 \\
\hline & Total & 19 & 16 & 3 & 10 & 10 & 0 \\
\hline \multirow[t]{4}{*}{ Ordnance } & High & 5 & 5 & 0 & 1 & 1 & 0 \\
\hline & Low & 11 & 8 & 3 & 6 & 3 & 3 \\
\hline & Medium & 7 & 6 & 1 & 1 & 1 & 0 \\
\hline & Total & 23 & 19 & 4 & 8 & 5 & 3 \\
\hline Total & & 135 & 85 & 50 & 107 & 63 & 44 \\
\hline
\end{tabular}




\begin{tabular}{|c|c|c|c|c|c|c|c|}
\hline \multicolumn{8}{|c|}{$\begin{array}{l}\text { Table C20 } \\
\text { Breakdown of Discrimination - QA Range and Area A - NRL without } \\
20 / 40 \mathrm{~mm}\end{array}$} \\
\hline \multirow[b]{3}{*}{$\begin{array}{l}\text { Ground- } \\
\text { truth }\end{array}$} & \multicolumn{7}{|c|}{ Demonstrator Classification } \\
\hline & \multirow[b]{2}{*}{ Confidence } & \multicolumn{3}{|c|}{ QA Range } & \multicolumn{3}{|c|}{ Area $\mathbf{A}$} \\
\hline & & \begin{tabular}{|l|} 
Total \\
Anomalies \\
\end{tabular} & \begin{tabular}{|l|} 
Declared \\
Ordnance \\
\end{tabular} & \begin{tabular}{|l|} 
Declared \\
Clutter \\
\end{tabular} & \begin{tabular}{|l|} 
Total \\
Anomalies
\end{tabular} & \begin{tabular}{|l|} 
Declared \\
Ordnance \\
\end{tabular} & \begin{tabular}{|l|} 
Declared \\
Clutter
\end{tabular} \\
\hline \multirow{4}{*}{$\begin{array}{l}\text { Non- } \\
\text { emplaced }\end{array}$} & High & 20 & 20 & 0 & 0 & 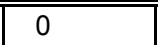 & 0 \\
\hline & Low & 318 & 146 & 172 & 93 & 41 & 52 \\
\hline & Medium & 188 & 61 & 127 & 61 & 20 & 41 \\
\hline & Total & 526 & 227 & 299 & 154 & 61 & 93 \\
\hline \multirow[t]{4}{*}{ Clutter } & High & 13 & 13 & 0 & 2 & 2 & 0 \\
\hline & Low & 29 & 17 & 12 & 7 & 6 & 1 \\
\hline & Medium & 14 & 13 & 1 & 3 & 3 & 0 \\
\hline & Total & 56 & 43 & 13 & 12 & 11 & 1 \\
\hline \multirow[t]{4}{*}{\begin{tabular}{|l|} 
Ordnance \\
\end{tabular}} & High & 11 & 11 & 0 & 2 & 2 & 0 \\
\hline & Low & 30 & 23 & 7 & 8 & 8 & 0 \\
\hline & Medium & 16 & 9 & 7 & 2 & 1 & 1 \\
\hline & Total & 57 & 43 & 14 & 12 & 11 & 1 \\
\hline Total & & 639 & 313 & 326 & 178 & 83 & 95 \\
\hline
\end{tabular}

\begin{tabular}{|c|c|c|c|c|c|c|c|}
\hline \multicolumn{8}{|c|}{$\begin{array}{l}\text { Table C21 } \\
\text { Breakdown of Discrimination - Area B and Area C - NRL without } \\
20 / 40 \mathrm{~mm}\end{array}$} \\
\hline \multirow[b]{3}{*}{$\begin{array}{l}\text { Ground- } \\
\text { truth }\end{array}$} & \multicolumn{7}{|c|}{ Demonstrator Classification } \\
\hline & \multirow[b]{2}{*}{ Confidence } & \multicolumn{3}{|c|}{ Area B } & \multicolumn{3}{|c|}{ Area C } \\
\hline & & \begin{tabular}{|l|} 
Total \\
Anomalies
\end{tabular} & $\begin{array}{l}\text { Declared } \\
\text { Ordnance }\end{array}$ & \begin{tabular}{|l|} 
Declared \\
Clutter
\end{tabular} & \begin{tabular}{|l|} 
Total \\
Anomalies
\end{tabular} & $\begin{array}{l}\text { Declared } \\
\text { Ordnance }\end{array}$ & $\begin{array}{l}\text { Declared } \\
\text { Clutter }\end{array}$ \\
\hline \multirow{4}{*}{$\begin{array}{l}\text { Non- } \\
\text { emplaced }\end{array}$} & High & 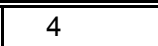 & 4 & 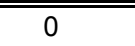 & 16 & 16 & 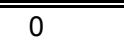 \\
\hline & Low & 147 & 64 & 83 & 78 & 41 & 37 \\
\hline & Medium & 82 & 25 & 57 & 45 & 16 & 29 \\
\hline & Total & 233 & 93 & 140 & 139 & 73 & 66 \\
\hline \multirow[t]{4}{*}{ Clutter } & High & 5 & 5 & 0 & 6 & 6 & 0 \\
\hline & Low & 17 & 8 & 9 & 5 & 3 & 2 \\
\hline & Medium & 7 & 6 & 1 & 4 & 4 & 0 \\
\hline & Total & 29 & 19 & 10 & 15 & 13 & 2 \\
\hline \multirow[t]{4}{*}{ Ordnance } & High & 6 & 6 & 0 & 3 & 3 & 0 \\
\hline & Low & 16 & 10 & 6 & 6 & 5 & 1 \\
\hline & Medium & 11 & 6 & 5 & 3 & 2 & 1 \\
\hline & \begin{tabular}{|l|} 
Total \\
\end{tabular} & 33 & 22 & 11 & 12 & 10 & 2 \\
\hline \begin{tabular}{|l|} 
Total \\
\end{tabular} & & 295 & 134 & 161 & 166 & 96 & 70 \\
\hline
\end{tabular}




\begin{tabular}{|c|c|c|c|c|c|c|c|}
\hline \multicolumn{8}{|c|}{$\begin{array}{l}\text { Table C22 } \\
\text { Breakdown of Discrimination - QA Range and Area A - Parsons } \\
\text { (EM-61) EM and Flag }\end{array}$} \\
\hline \multirow[b]{3}{*}{$\begin{array}{l}\text { Ground- } \\
\text { truth }\end{array}$} & \multicolumn{7}{|c|}{ Demonstrator Classification } \\
\hline & \multirow[b]{2}{*}{ Confidence } & \multicolumn{3}{|c|}{ QA Range } & \multicolumn{3}{|c|}{ Area A } \\
\hline & & \begin{tabular}{|l|} 
Total \\
Anomalies
\end{tabular} & \begin{tabular}{|l|} 
Declared \\
Ordnance
\end{tabular} & $\begin{array}{l}\text { Declared } \\
\text { Clutter }\end{array}$ & $\begin{array}{l}\text { Total } \\
\text { Anomalies }\end{array}$ & $\begin{array}{l}\text { Declared } \\
\text { Ordnance }\end{array}$ & $\begin{array}{l}\text { Declared } \\
\text { Clutter }\end{array}$ \\
\hline \multirow{5}{*}{$\begin{array}{l}\text { Non- } \\
\text { emplaced }\end{array}$} & Undeclared & 746 & 740 & 6 & 114 & 113 & 1 \\
\hline & High & 8 & 1 & 7 & 1 & 0 & 1 \\
\hline & Low & 1 & 0 & 1 & 1 & 0 & 1 \\
\hline & Medium & 2 & 2 & 0 & 1 & 1 & 0 \\
\hline & Total & 757 & 743 & 14 & 117 & 114 & 3 \\
\hline \multirow[t]{5}{*}{ Clutter } & Undeclared & 89 & 89 & 0 & 20 & 20 & 0 \\
\hline & High & 1 & 0 & 1 & 1 & 0 & 1 \\
\hline & Low & 0 & 0 & 0 & 0 & 0 & 0 \\
\hline & Medium & 1 & 1 & 0 & 0 & 0 & 0 \\
\hline & Total & 91 & 90 & 1 & 21 & 20 & 1 \\
\hline \multirow[t]{5}{*}{ Ordnance } & Undeclared & 88 & 88 & 0 & 18 & 18 & 0 \\
\hline & High & 0 & 0 & 0 & 0 & 0 & 0 \\
\hline & Low & 1 & 1 & 0 & 0 & 0 & 0 \\
\hline & Medium & 1 & 1 & 0 & 0 & 0 & 0 \\
\hline & Total & 90 & 90 & 0 & 18 & 18 & 0 \\
\hline Total & & 938 & 923 & 15 & 156 & 152 & 4 \\
\hline
\end{tabular}

\begin{tabular}{|c|c|c|c|c|c|c|c|}
\hline $\begin{array}{l}\text { Table C } \\
\text { Breakd } \\
\text { Flag } \\
\end{array}$ & $\begin{array}{l}23 \\
\text { own of } \mathrm{Di}\end{array}$ & crimina & $n-A r$ & $\mathbf{B}-\mathbf{P a}$ & rsons (E & 1-61) EM & and \\
\hline & & & Demonstr & ator Class & ification & & \\
\hline & & & Area B & & & Area C & \\
\hline $\begin{array}{l}\text { Ground- } \\
\text { truth }\end{array}$ & Confidence & \begin{tabular}{|l|} 
Total \\
Anomalies
\end{tabular} & \begin{tabular}{|l|} 
Declared \\
Ordnance
\end{tabular} & \begin{tabular}{|l|} 
Declared \\
Clutter
\end{tabular} & \begin{tabular}{|l|} 
Total \\
Anomalies
\end{tabular} & \begin{tabular}{|l|} 
Declared \\
Ordnance \\
\end{tabular} & \begin{tabular}{|l} 
Declared \\
Clutter
\end{tabular} \\
\hline Non- & Undeclared & 311 & 306 & 5 & 321 & 321 & 0 \\
\hline emplaced & High & 4 & 1 & 3 & 3 & 0 & 3 \\
\hline & Low & 0 & 0 & 0 & 0 & 0 & 0 \\
\hline & Medium & 1 & 1 & 0 & 0 & 0 & 0 \\
\hline & Total & 316 & 308 & 8 & 324 & 321 & 3 \\
\hline Clutter & Undeclared & 40 & 40 & 0 & 29 & 29 & 0 \\
\hline & High & 0 & 0 & 0 & 0 & 0 & 0 \\
\hline & Low & 0 & 0 & 0 & 0 & 0 & 0 \\
\hline & Medium & 1 & 1 & 0 & 0 & 0 & 0 \\
\hline & Total & 41 & 41 & 0 & 29 & 29 & 0 \\
\hline Ordnance & Undeclared & 48 & 48 & 0 & 22 & 22 & 0 \\
\hline & High & 0 & 0 & 0 & 0 & 0 & 0 \\
\hline & Low & 1 & 1 & 0 & 0 & 0 & 0 \\
\hline & Medium & 1 & 1 & 0 & 0 & 0 & 0 \\
\hline & Total & 50 & 50 & 0 & 22 & 22 & 0 \\
\hline Total & & 407 & 399 & 8 & 375 & 372 & 3 \\
\hline
\end{tabular}




\begin{tabular}{|c|c|c|c|c|c|c|c|}
\hline \multicolumn{8}{|c|}{\begin{tabular}{|l} 
Table C24 \\
Breakdown of Discrimination - QA Range and Area A - Parsons \\
EM-61 Digital
\end{tabular}} \\
\hline \multirow[b]{3}{*}{$\begin{array}{l}\text { Ground- } \\
\text { truth }\end{array}$} & \multicolumn{7}{|c|}{ Demonstrator Classification } \\
\hline & \multirow[b]{2}{*}{ Confidence } & \multicolumn{3}{|c|}{ QA Range } & \multicolumn{3}{|c|}{ Area A } \\
\hline & & \begin{tabular}{|l|} 
Total \\
Anomalies
\end{tabular} & $\begin{array}{l}\text { Declared } \\
\text { Ordnance }\end{array}$ & $\begin{array}{l}\text { Declared } \\
\text { Clutter }\end{array}$ & $\begin{array}{l}\text { Total } \\
\text { Anomalies }\end{array}$ & \begin{tabular}{|l|} 
Declared \\
Ordnance
\end{tabular} & \begin{tabular}{|l} 
Declared \\
Clutter
\end{tabular} \\
\hline \multirow{4}{*}{$\begin{array}{l}\text { Non- } \\
\text { emplaced }\end{array}$} & High & 221 & 83 & 138 & 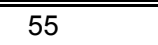 & 16 & 39 \\
\hline & Low & 107 & 0 & 107 & 61 & 0 & 61 \\
\hline & Medium & 1,091 & 1 & 1,090 & 252 & 1 & 251 \\
\hline & Total & 1,419 & 84 & 1,335 & 368 & 17 & 351 \\
\hline \multirow[t]{4}{*}{ Clutter } & High & 49 & 29 & 20 & 16 & 11 & 5 \\
\hline & Low & 3 & 0 & 3 & 2 & 0 & 2 \\
\hline & Medium & 59 & 0 & 59 & 6 & 0 & 6 \\
\hline & Total & 111 & 29 & 82 & 24 & 11 & 13 \\
\hline \multirow[t]{4}{*}{\begin{tabular}{|l|} 
Ordnance \\
\end{tabular}} & High & 49 & 33 & 16 & 11 & 6 & 5 \\
\hline & Low & 1 & 0 & 1 & 1 & 0 & 1 \\
\hline & Medium & 40 & 0 & 40 & 6 & 0 & 6 \\
\hline & Total & 90 & 33 & 57 & 18 & 6 & 12 \\
\hline Total & & 1,620 & 146 & 1,474 & 410 & 34 & 376 \\
\hline
\end{tabular}

\begin{tabular}{|c|c|c|c|c|c|c|c|}
\hline \multicolumn{8}{|c|}{$\begin{array}{l}\text { Table C25 } \\
\text { Breakdown of Discrimination - Area B and Area C - Parsons EM-61 } \\
\text { Digital }\end{array}$} \\
\hline \multirow[b]{3}{*}{$\begin{array}{l}\text { Ground- } \\
\text { truth }\end{array}$} & \multicolumn{7}{|c|}{ Demonstrator Classification } \\
\hline & \multirow[b]{2}{*}{ Confidence } & \multicolumn{3}{|c|}{ Area B } & \multicolumn{3}{|c|}{ Area C } \\
\hline & & \begin{tabular}{|l} 
Total \\
Anomalies
\end{tabular} & $\begin{array}{l}\text { Declared } \\
\text { Ordnance }\end{array}$ & $\begin{array}{l}\text { Declared } \\
\text { Clutter }\end{array}$ & \begin{tabular}{|l} 
Total \\
Anomalies
\end{tabular} & $\begin{array}{l}\text { Declared } \\
\text { Ordnance }\end{array}$ & $\begin{array}{l}\text { Declared } \\
\text { Clutter }\end{array}$ \\
\hline \multirow{4}{*}{$\begin{array}{l}\text { Non- } \\
\text { emplaced }\end{array}$} & High & ב63 & 34 & 29 & 103 & 33 & 70 \\
\hline & Low & 3 & 0 & 3 & 43 & 0 & 43 \\
\hline & Medium & 423 & 0 & 423 & 416 & 0 & 416 \\
\hline & \begin{tabular}{|l|} 
Total \\
\end{tabular} & 489 & 34 & 455 & 562 & 33 & 529 \\
\hline \multirow[t]{4}{*}{ Clutter } & High & 19 & 8 & 11 & 14 & 10 & 4 \\
\hline & Low & 0 & 0 & 0 & 1 & 0 & 1 \\
\hline & Medium & 36 & 0 & 36 & 17 & 0 & 17 \\
\hline & Total & 55 & 8 & 47 & 32 & 10 & 22 \\
\hline \multirow[t]{4}{*}{ Ordnance } & High & 26 & 18 & 8 & 12 & 9 & 3 \\
\hline & Low & 0 & 0 & 0 & 0 & 0 & 0 \\
\hline & Medium & 23 & 0 & 23 & 11 & 0 & 11 \\
\hline & Total & 49 & 18 & 31 & 23 & 9 & 14 \\
\hline Total & & 593 & 60 & 533 & 617 & 52 & 565 \\
\hline
\end{tabular}




\begin{tabular}{|c|c|c|c|c|c|c|c|}
\hline \multicolumn{8}{|c|}{$\begin{array}{l}\text { Table C26 } \\
\text { Breakdown of Discrimination - QA Range and Area A - Parsons } \\
\text { TM-5 EMU }\end{array}$} \\
\hline \multirow[b]{3}{*}{$\begin{array}{l}\text { Ground- } \\
\text { truth }\end{array}$} & \multicolumn{7}{|c|}{ Demonstrator Classification } \\
\hline & \multirow[b]{2}{*}{ Confidence } & \multicolumn{3}{|c|}{ QA Range } & \multicolumn{3}{|c|}{ Area A } \\
\hline & & \begin{tabular}{|l|} 
Total \\
Anomalies \\
\end{tabular} & \begin{tabular}{|l|} 
Declared \\
Ordnance
\end{tabular} & \begin{tabular}{|l|} 
Declared \\
Clutter
\end{tabular} & \begin{tabular}{|l|} 
Total \\
Anomalies
\end{tabular} & $\begin{array}{l}\text { Declared } \\
\text { Ordnance }\end{array}$ & \begin{tabular}{|l|} 
Declared \\
Clutter \\
\end{tabular} \\
\hline \multirow{3}{*}{$\begin{array}{l}\text { Non- } \\
\text { emplaced }\end{array}$} & Undeclared & 120 & 116 & 4 & 2 & 2 & 0 \\
\hline & High & 3 & 1 & 2 & 0 & 0 & 0 \\
\hline & Total & 123 & 117 & 6 & 2 & 2 & 0 \\
\hline \multirow[t]{3}{*}{ Clutter } & Undeclared & 38 & 38 & 0 & 8 & 8 & 0 \\
\hline & High & 0 & 0 & 0 & 0 & 0 & 0 \\
\hline & Total & 38 & 38 & 0 & 8 & 8 & 0 \\
\hline \multirow[t]{3}{*}{ Ordnance } & Undeclared & 33 & 33 & 0 & 7 & 7 & 0 \\
\hline & High & 0 & 0 & 0 & 0 & 0 & 0 \\
\hline & Total & 33 & 33 & 0 & 7 & 7 & 0 \\
\hline \begin{tabular}{|l} 
Total \\
\end{tabular} & & 194 & 188 & 6 & 17 & 17 & 0 \\
\hline
\end{tabular}

\begin{tabular}{|c|c|c|c|c|c|c|c|}
\hline \multicolumn{8}{|c|}{$\begin{array}{l}\text { Table C27 } \\
\text { Breakdown of Discrimination - Area B and Area C - Parsons TM-5 } \\
\text { EMU }\end{array}$} \\
\hline \multirow[b]{3}{*}{$\begin{array}{l}\text { Ground- } \\
\text { truth }\end{array}$} & \multicolumn{7}{|c|}{ Demonstrator Classification } \\
\hline & \multirow[b]{2}{*}{ Confidence } & \multicolumn{3}{|c|}{ Area B } & \multicolumn{3}{|c|}{ Area C } \\
\hline & & \begin{tabular}{|l|} 
Total \\
Anomalies \\
\end{tabular} & $\begin{array}{l}\text { Declared } \\
\text { Ordnance } \\
\end{array}$ & \begin{tabular}{|l|} 
Declared \\
Clutter
\end{tabular} & \begin{tabular}{|l|} 
Total \\
Anomalies \\
\end{tabular} & $\begin{array}{l}\text { Declared } \\
\text { Ordnance }\end{array}$ & $\begin{array}{l}\text { Declared } \\
\text { Clutter }\end{array}$ \\
\hline \multirow{3}{*}{$\begin{array}{l}\text { Non- } \\
\text { emplaced }\end{array}$} & Undeclared & 102 & 99 & 3 & 16 & 15 & $\overline{1}$ \\
\hline & High & 3 & 1 & 2 & 0 & 0 & 0 \\
\hline & Total & 105 & 100 & 5 & 16 & 15 & 1 \\
\hline \multirow[t]{3}{*}{ Clutter } & Undeclared & 26 & 26 & 0 & 4 & 4 & 0 \\
\hline & High & 0 & 0 & 0 & 0 & 0 & 0 \\
\hline & Total & 26 & 26 & 0 & 4 & 4 & 0 \\
\hline \multirow[t]{3}{*}{ Ordnance } & Undeclared & 22 & 22 & 0 & 4 & 4 & 0 \\
\hline & High & 0 & 0 & 0 & 0 & 0 & 0 \\
\hline & Total & 22 & 22 & 0 & 4 & 4 & 0 \\
\hline Total & & 153 & 148 & 5 & 24 & 23 & 1 \\
\hline
\end{tabular}

\section{Identification results}

The UXO identification performance of each demonstrator is based on the UXO type declarations included in each of the required prioritized dig lists. The results presented in this section have been adjusted to account for UXO-related items that were present in Areas A, B, and C from previous demonstration work. Figures $\mathrm{C} 1$ through $\mathrm{C} 5$ include classification matrices that detail and summarize the identification performance of each demonstrator. There are two types of classification matrices presented.

The first type of classification matrix is the target classification matrix, which shows how well the demonstrator identified specific ordnance types that were detected. This classification matrix includes the following entries: 
a. The entry below the demonstrator's name indicates the demonstration Area. Area A is the four 30-m grid, Area B is the 1-hectare site, and Area $\mathrm{C}$ is the six $30-\mathrm{m}$ grid.

$b$. The classes across the top of the matrix are the classification types of the actual target classes. A list of these types was given to the demonstrators for use in classifying their detected ordnance. A clutter class is listed as a separate entry and corresponds to a false alarm if classified as a UXO target and to a correct discrimination when classified as non-UXO by the demonstrator.

c. The classification column on the left side of the matrix lists the demonstrator's declaration for each detected UXO item.

d. The totals in the right-hand column of the matrix correspond to the total number of items declared by the demonstrator as a particular class.

e. The "\% Classified" row indicates the percentage of detected targets, for a given target class, that were correctly classified.

$f$. The "\% Classified by Class" indicates the percentage of detected targets, for the given classes of projectile, mortar, rocket, and practice bomb, that were correctly classified.

g. The "\% of Total Detected Targets Classified as Nonordnance Low/Med Confidence" is the percentage of detected ordnance that the demonstrator incorrectly classified as nonordnance with low or medium confidence.

$h$. The "\% of Total Detected Targets Classified as Nonordnance High Confidence" is the percentage of detected ordnance that the demonstrator incorrectly classified as nonordnance with high confidence. This classification error carried the highest cost penalty (equivalent to the cost of a complete resurvey of the area).

Note that only one demonstrator classified their declarations with the given target classes.

The second type of classification matrix is the aggregate size classification matrix. This matrix includes the following entries:

a. The entry below the demonstrator's name indicates the demonstration Area.

$b$. The classes across the top of the matrix are the aggregate size classes of the items emplaced. The targets are grouped into three classes. The small target class contains the $20-\mathrm{mm}$ projectile, the $40-\mathrm{mm}$ projectile, and the BDU 3 practice bomb. The medium class contains the $60-\mathrm{mm}$ mortar, the 81-mm mortar, the 2.25-in. rocket, the 2.75-in. rocket warhead, the SMAW rocket, the LAAW, the BDU-33 practice bomb, the MK-3 practice bomb, and the MK-106 practice bomb. The large class contains the 5-in. projectile; the $105-\mathrm{mm}$ projectile, the MK- 81 practice bomb, the MK- 82 practice bomb, and the MK-83 practice bomb. A clutter class is listed as a separate entry and corresponds to a false alarm if classified as a UXO target and to a correct discrimination when classified as non$\mathrm{UXO}$ by the demonstrator. 
c. The classification column on the left side of the matrix lists the demonstrator's declaration for each detected UXO item. Again, the declarations are grouped into three aggregate size classes as previously described in the target classes.

d. The totals in the right-hand column of the matrix correspond to the total number of items declared by the demonstrator as a particular class.

e. The "\% Classified" row indicates the percentage of detected targets, for a given aggregate size class, that were correctly classified.

f. The "\% of Total Detected Targets Classified as Nonordnance Low/Med Confidence" is the percentage of detected ordnance that the demonstrator incorrectly classified as nonordnance with low or medium confidence.

g. The " $\%$ of Total Detected Targets Classified as Nonordnance High Confidence" is the percentage of detected ordnance that the demonstrator incorrectly classified as nonordnance with high confidence. Again, this classification error carried the highest cost penalty (equivalent to the cost of a complete resurvey of the area).

Note that only two demonstrators classified their declarations with enough descriptive information to allow for the evaluation of aggregate size classification.

NAEVA was the only demonstrator that submitted specific target identifications. Again, as with the $0.5-\mathrm{m}$ analysis, their classification results, shown in Figures $\mathrm{C} 1$ through $\mathrm{C} 3$, were very poor. Their results are only slightly better when aggregated by size, as shown in Figure $\mathrm{C} 4$ for detections within $1.0 \mathrm{~m}$. Classification matrices aggregated by size for Geophex are shown in Figure C5 for detections within $1.0 \mathrm{~m}$. Geophex correctly classified a few more ordnance by size than NAEVA, but also classified more non-ordnance items as ordnance. NAEVA and Geophex are the only demonstrators who attempted to identify ordnance by size.

\section{ROC-Based Performance Assessment}

\section{Assessment of detection, discrimination, and false alarm rate performance for $1.0 \mathrm{~m}$}

Figures $\mathrm{C} 6, \mathrm{C} 7$, and $\mathrm{C} 8$ show the demonstrators' detection performance for Areas A, B, and C, respectively. The distance threshold for scoring a detection is set to $1.0 \mathrm{~m}$ for these sets of plots. Figure C6 shows that in Area A, all of the systems operated along very similar ROC curves, with the major difference being their selection of the "stop dig" or end point threshold. In the cases of NRL and Parsons TM-5 EMU, the endpoint thresholds were set so high that the operating (and maximum achievable) P(det) was much lower than those of the other demonstrators. Geophex achieved the best performance for this area, but it is apparent from the steep slope of the ROC curve, that a lower endpoint threshold would probably have resulted in increased $\mathrm{P}(\mathrm{det})$ with a relatively small increase in total FAR. 


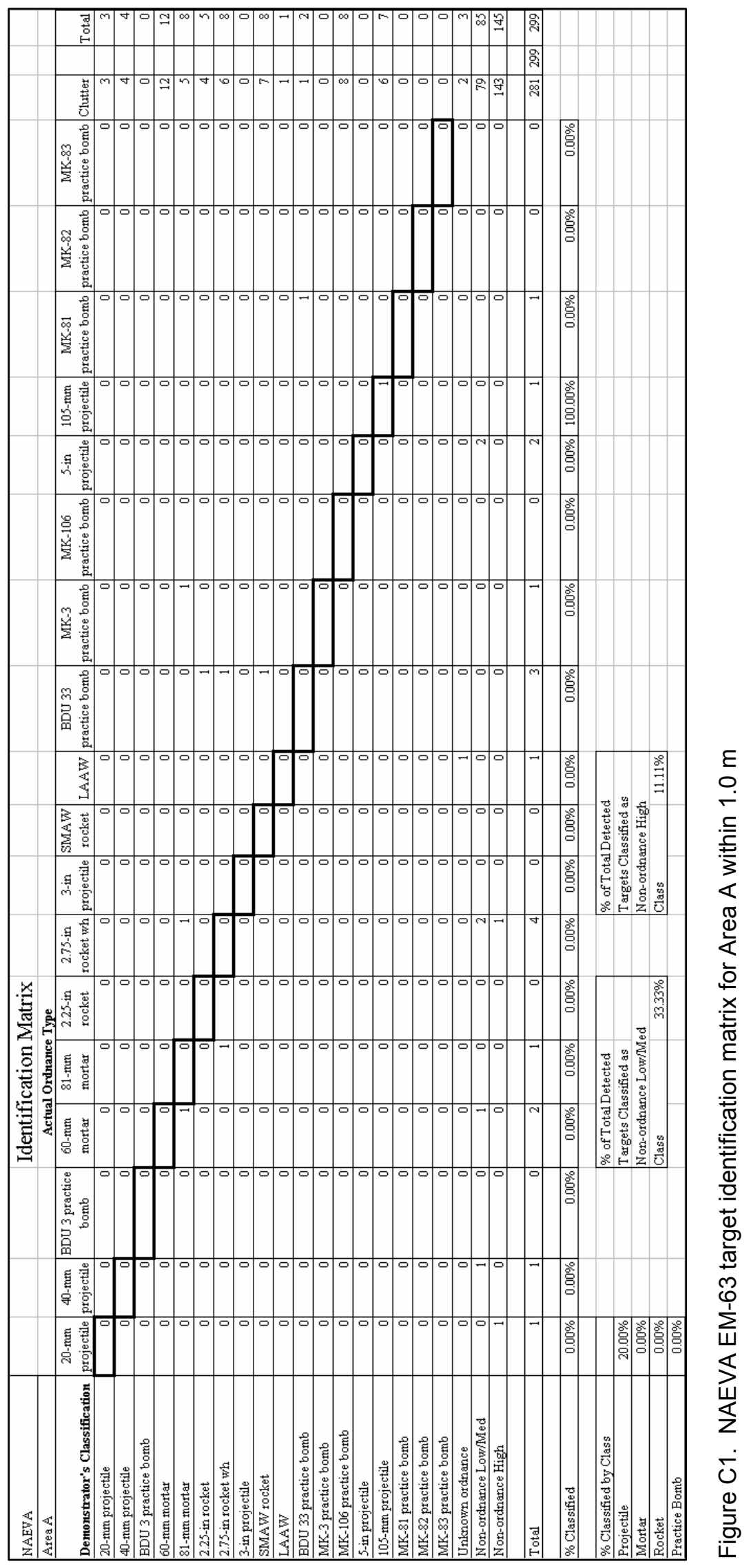




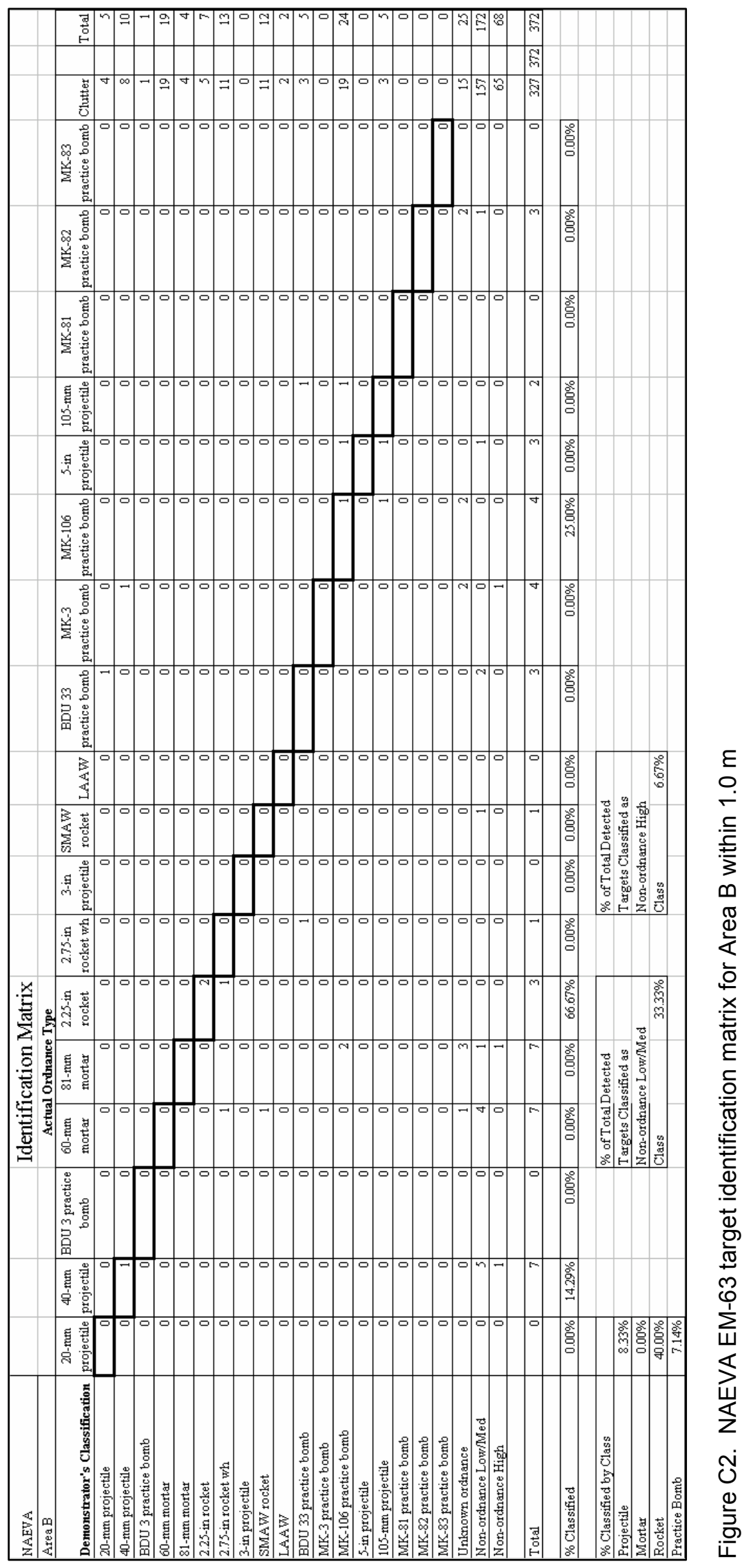




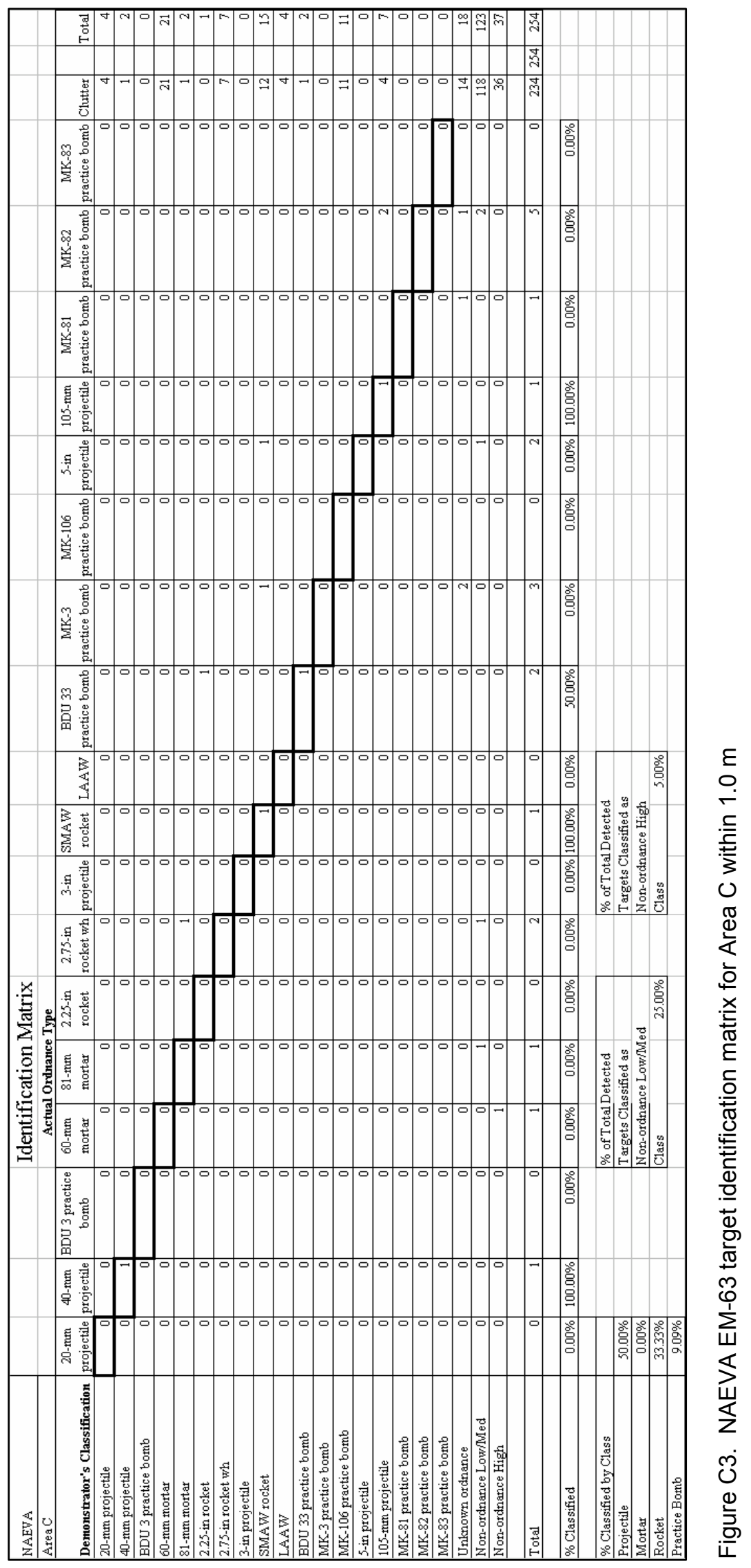




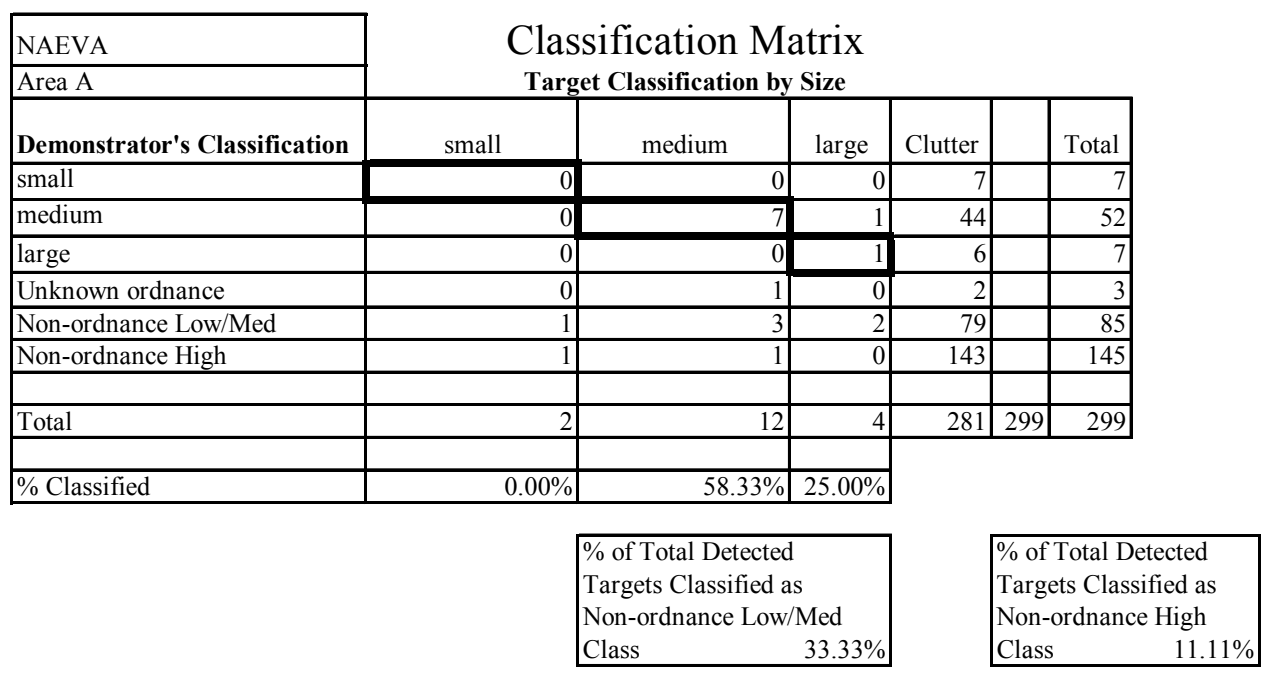

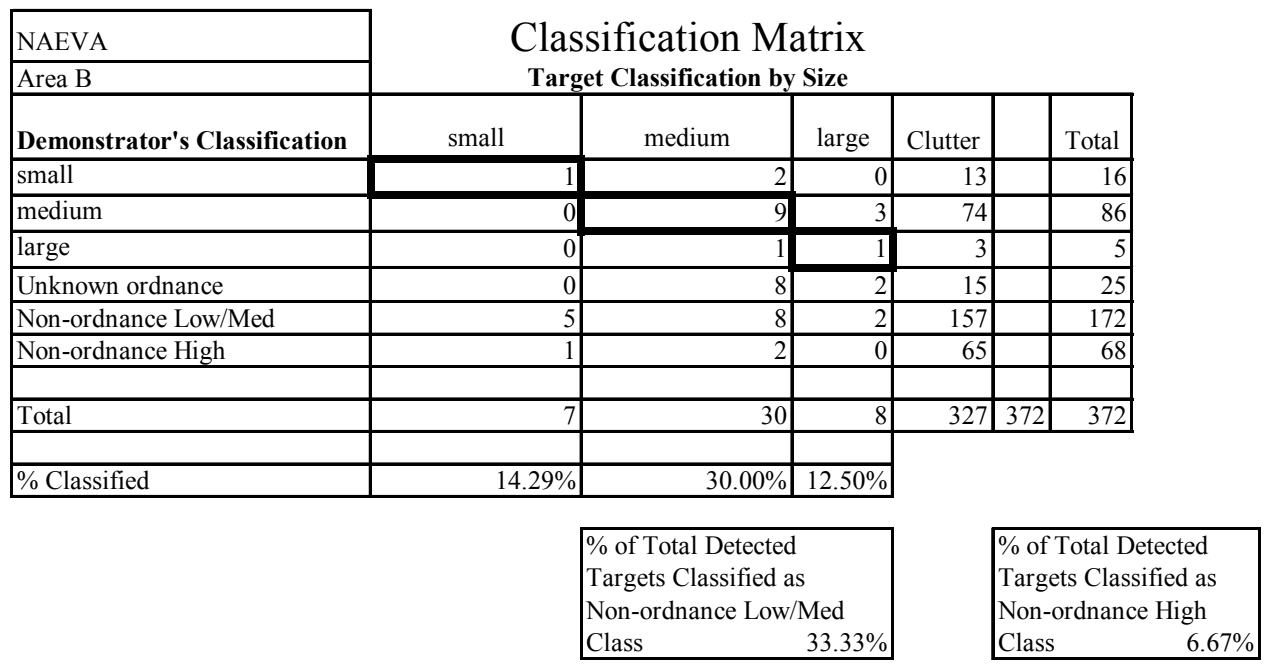

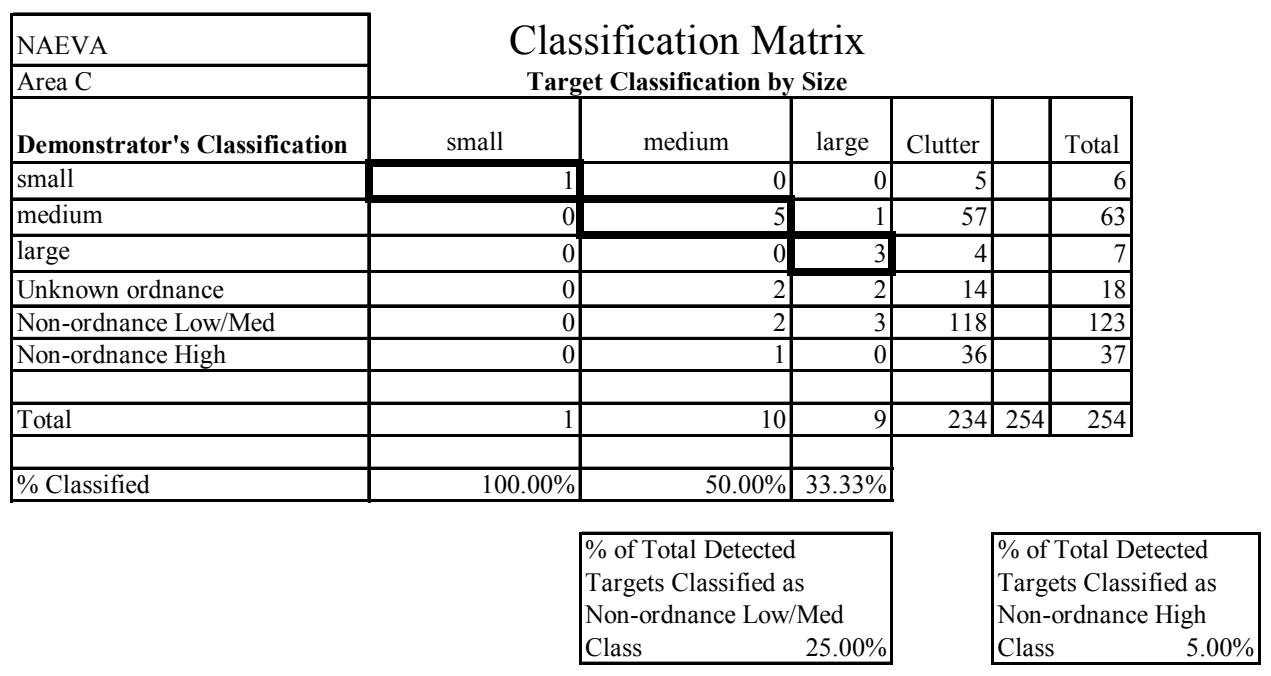

Figure C4. NAEVA EM-63 size classification matrix within $1.0 \mathrm{~m}$ 


\begin{tabular}{|c|c|c|c|c|c|c|c|}
\hline \multirow{3}{*}{\begin{tabular}{|l|} 
Geophex \\
Area A \\
Demonstrator's Classification
\end{tabular}} & \multirow{2}{*}{\multicolumn{6}{|c|}{$\begin{array}{c}\text { Classification Matrix } \\
\text { Target Classification by Size }\end{array}$}} & \\
\hline & & & & & & & \\
\hline & small & medium & large & Clutter & \multicolumn{2}{|r|}{ Total } & \\
\hline small & 2 & 1 & 0 & 23 & & 26 & \\
\hline medium & 0 & 9 & 2 & 53 & & 64 & \\
\hline large & 1 & 3 & 1 & 45 & & 50 & \\
\hline Unknown ordnance & 0 & 0 & 0 & 0 & & 0 & \\
\hline Non-ordnance Low/Med & 0 & 0 & 1 & 9 & & 10 & \\
\hline Non-ordnance High & 0 & 0 & 0 & 0 & & 0 & \\
\hline Total & 3 & 13 & 4 & 130 & 150 & 150 & \\
\hline \multirow[t]{3}{*}{$\%$ Classified } & $66.67 \%$ & $69.23 \%$ & $25.00 \%$ & & & & \\
\hline & & \multicolumn{2}{|c|}{$\begin{array}{l}\% \text { of Total Detected } \\
\text { Targets Classified as } \\
\text { Non-ordnance Low/Med }\end{array}$} & & \multicolumn{3}{|c|}{$\begin{array}{l}\% \text { of Total Detected } \\
\text { Targets Classified as } \\
\text { Non-ordnance High }\end{array}$} \\
\hline & & Class & $5.00 \%$ & & Class & & $0.00 \%$ \\
\hline
\end{tabular}

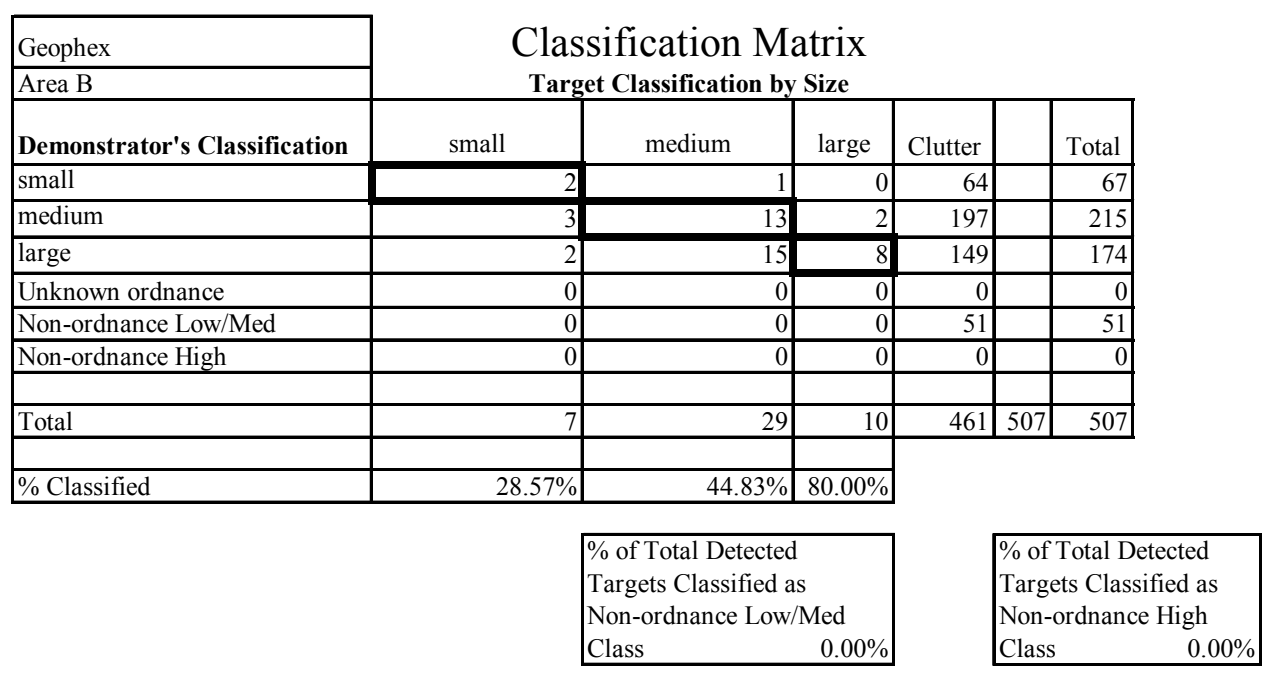

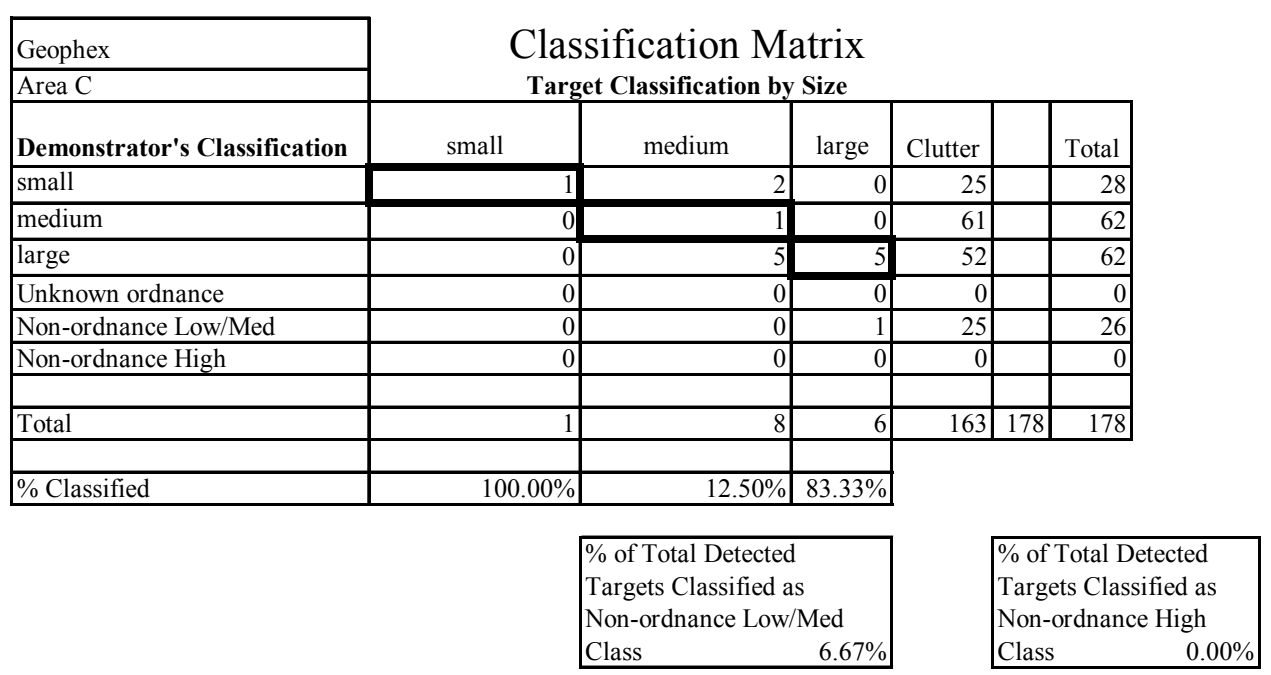

Figure C5. Geophex, Ltd. GEM-3 size classification matrix within $1.0 \mathrm{~m}$ 


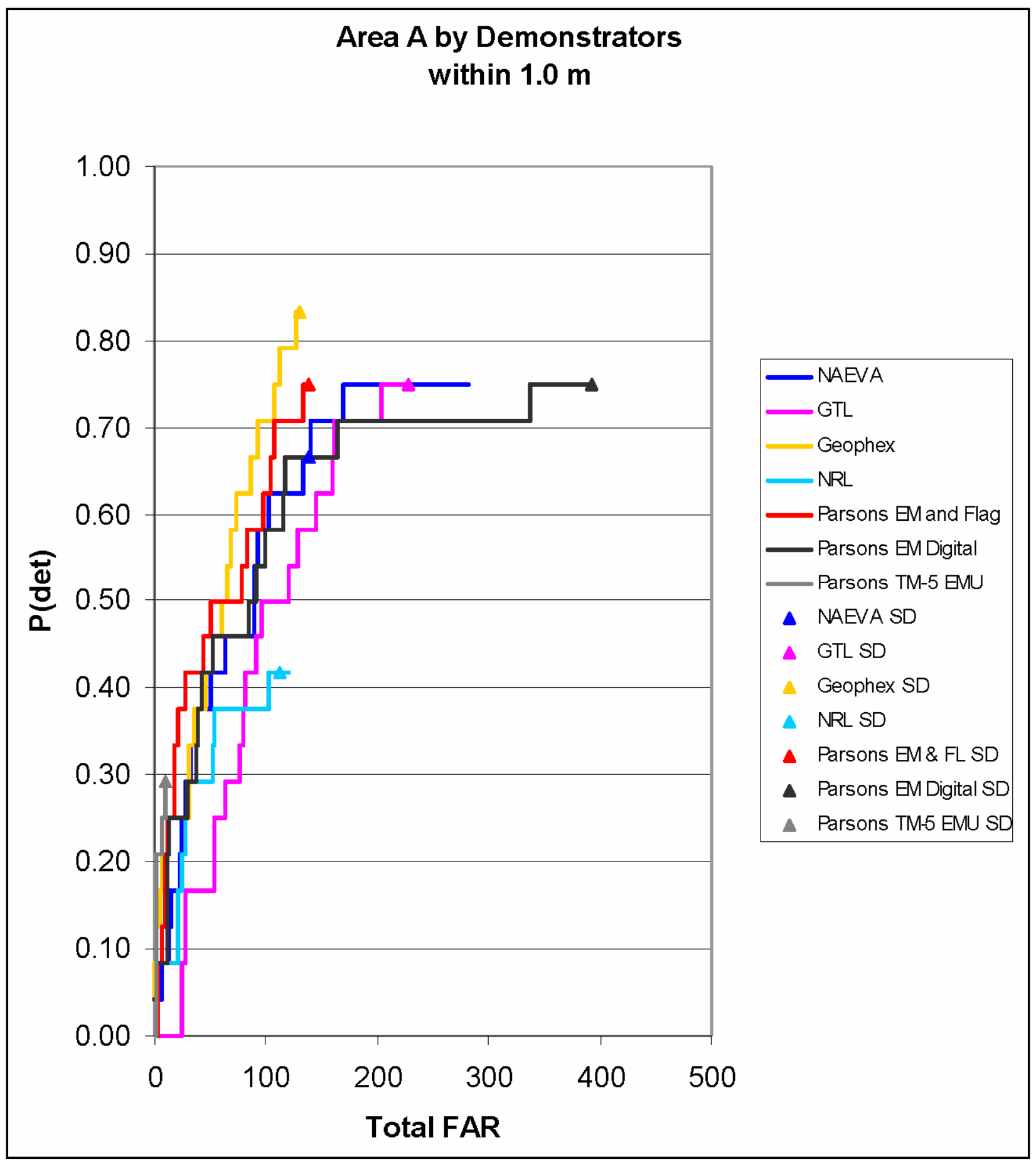

Figure C6. Area $A-P($ det) vs. total FAR within $1.0 \mathrm{~m}$ for all demonstrators 


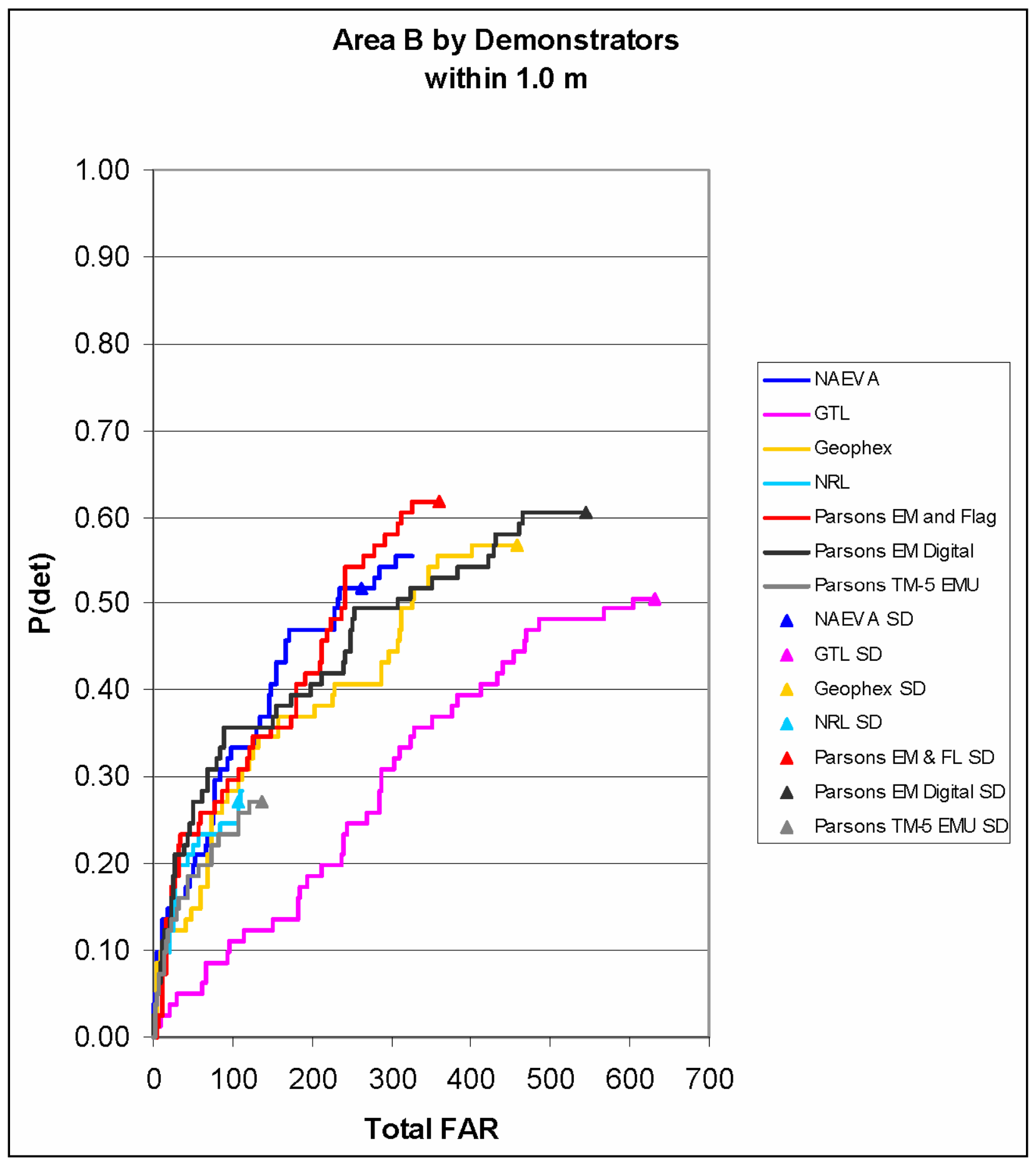

Figure $C 7$. Area $B-P($ det) vs. total $F A R$ within $1.0 \mathrm{~m}$ for all demonstrators 


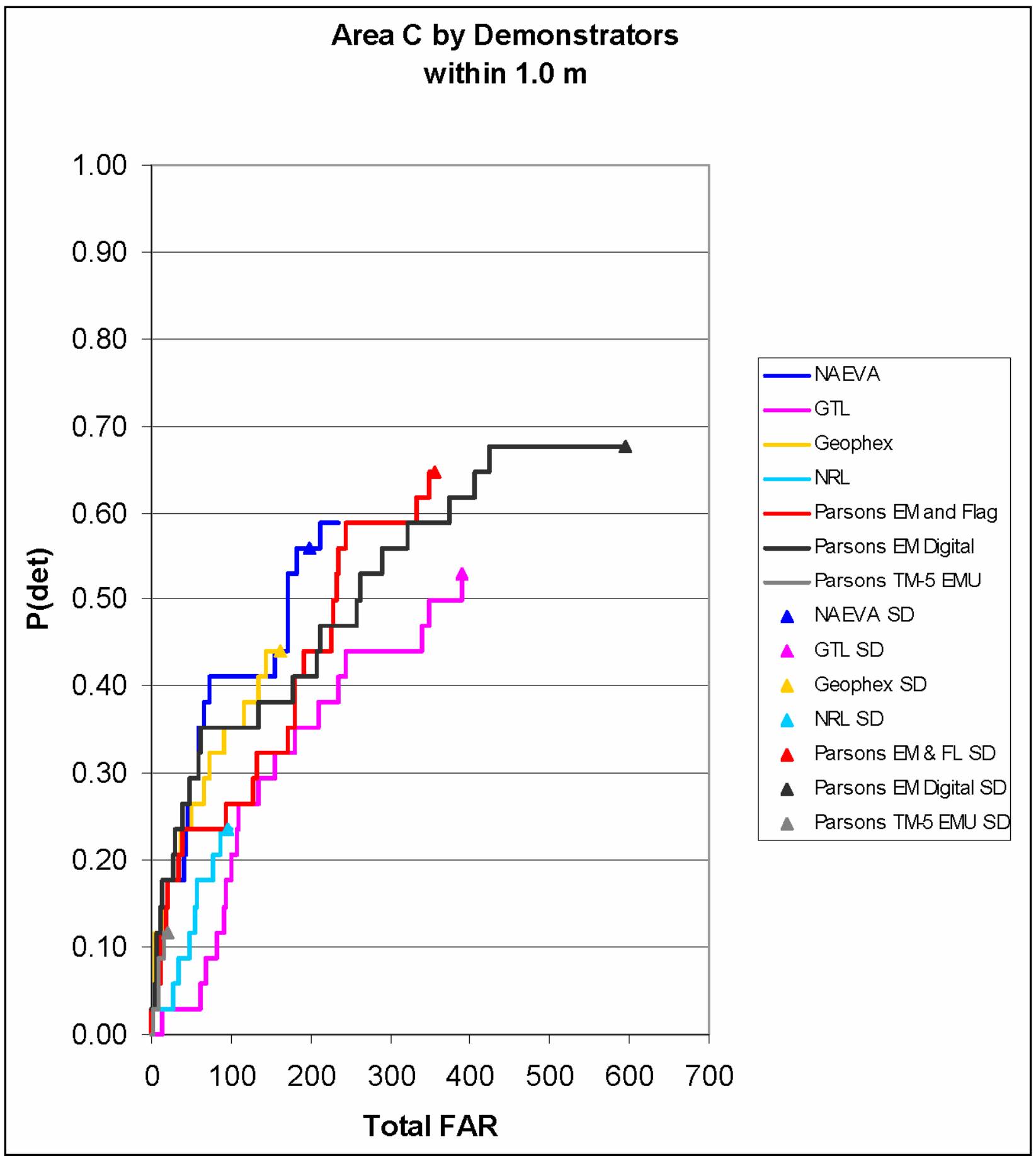

Figure $\mathrm{C} 8$. Area $\mathrm{C}-\mathrm{P}$ (det) vs. total $\mathrm{FAR}$ within $1.0 \mathrm{~m}$ for all demonstrators 
The placement of the "stop dig" point, (shown as the "SD" triangle in each of these figures) indicates the demonstrators' general lack of confidence in their discrimination capability. With the exception of NAEVA, all other demonstrators placed their "stop dig" point at the end of the dig list. NAEVA's attempt at discrimination resulted in an operating $\mathrm{P}(\mathrm{det})$ that was 10 percent lower than the maximum achievable.

Figure $\mathrm{C} 7$ shows that all demonstrators, with the exception of GTL, achieved very similar ROC-based performance. GTL demonstrated significantly poorer detection and total FAR performance than the others. Overall, all of the systems demonstrated poorer ROC-based performance in Area B than Area A, confirming previous reports that Area B contained significantly higher levels of geologic anomalies and metallic clutter. As in Area A, all demonstrators with the exception of NAEVA, were extremely conservative in their "stop dig" point selection.

Figure $\mathrm{C} 8$ shows that all of the demonstrators operated along very similar ROC curves. Again, all of the threshold selections were very conservative. With the exception of Geophex and NRL, all other demonstrators performed better in area $\mathrm{C}$ than $\mathrm{B}$, but none performed better than in Area $\mathrm{A}$.

Figures $\mathrm{C} 9$ through $\mathrm{C} 11$ show the results of reanalyzing the data to include only targets larger than $40 \mathrm{~mm}$ projectiles. Only NRL and Geophex performed this analysis and resubmitted revised dig lists. These figures show that NRL and Geophex achieve 10 percent and 7 percent higher $\mathrm{P}(\mathrm{det})$, respectively in Area A when the small targets are ignored. Considerably higher improvements of 30 percent and 15 percent, respectively, were achieved in Area B, while only 15 percent and 6 percent improvements were achieved in Area C.

The small number of submissions, and the lack of this type of data from Parsons precludes any comparisons across systems or with a baseline. In order to overcome this deficiency, the demonstrators dig lists that contained all targets (including 20 and $40 \mathrm{~mm}$ ) were evaluated with the $20 / 40 \mathrm{~mm}$ targets removed from the groundtruth. No detection, false alarm, or missed target was assigned to any declaration within $1.0 \mathrm{~m}$ of these small emplaced targets. Figures C12 through $\mathrm{C} 14$ show the results of this evaluation. These ROC curves show a slight improvement in performance for all demonstrators, but no significant change in ROC curve shape or in the relative performance between demonstrators. It can be concluded that all systems have similar capability (or lack thereof) to detect the smaller targets in the Kaho'olawe environment.

Figures $\mathrm{C} 15$ through $\mathrm{C} 17$ show the $\mathrm{P}(\mathrm{det})$ performance as a function of $\mathrm{Pfp}$ for all demonstrators, where Pfp is computed as the ratio of the number of emplaced clutter items included on the dig list to the number of clutter items emplaced. This metric attempted to separate the effects of the geology and unknown metal clutter from those from known, emplaced clutter items. These ROC curves show very small differences between demonstrators, but most importantly, the almost consistently flat, diagonal shape of the curves indicates that none of the systems demonstrated a capability to discriminate emplaced UXO from emplaced metallic clutter in this environment. 


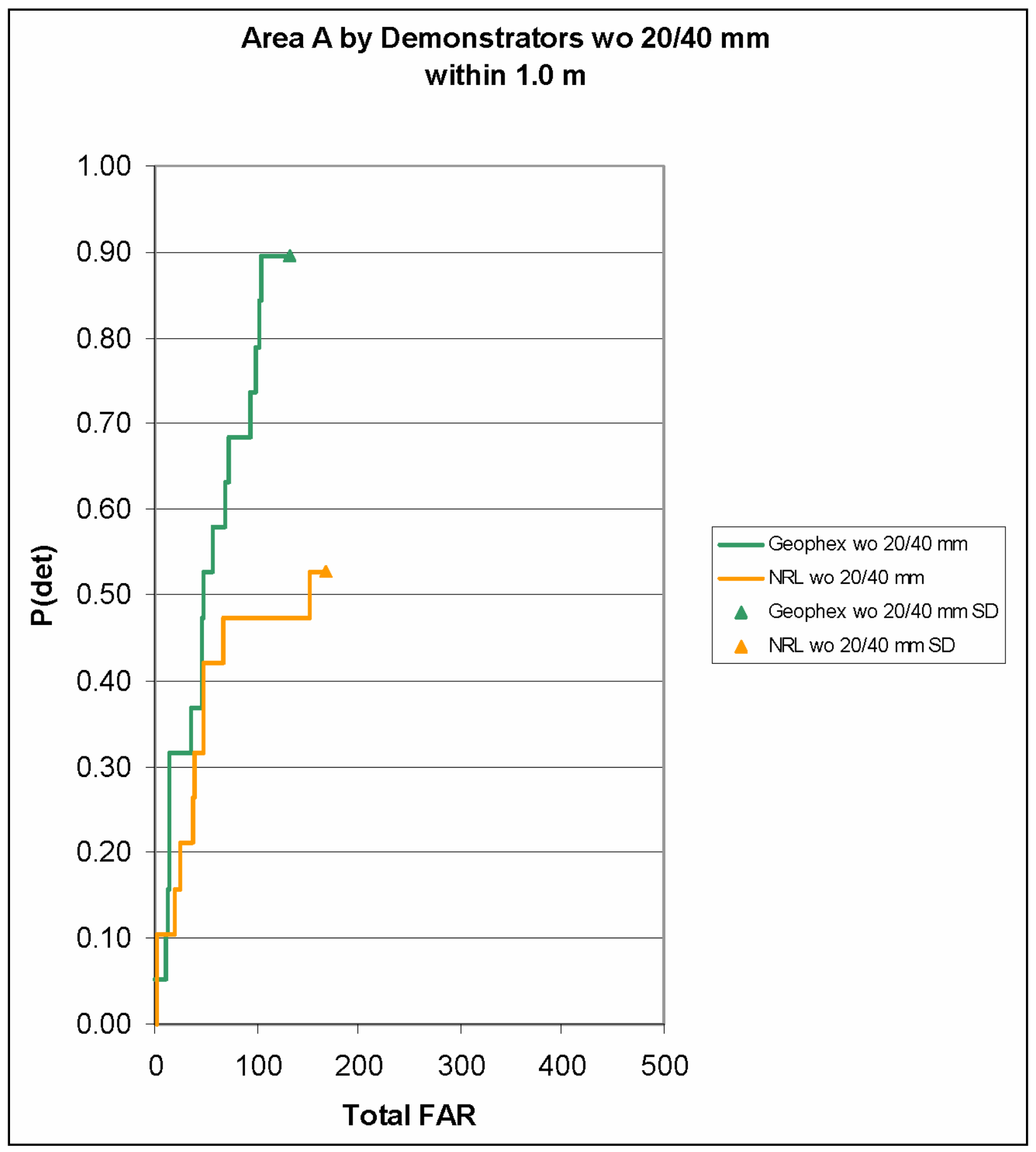

Figure C9. Area A - P(det) vs. total FAR within $1.0 \mathrm{~m}$ for demonstrators without (wo) 20/40 mm 


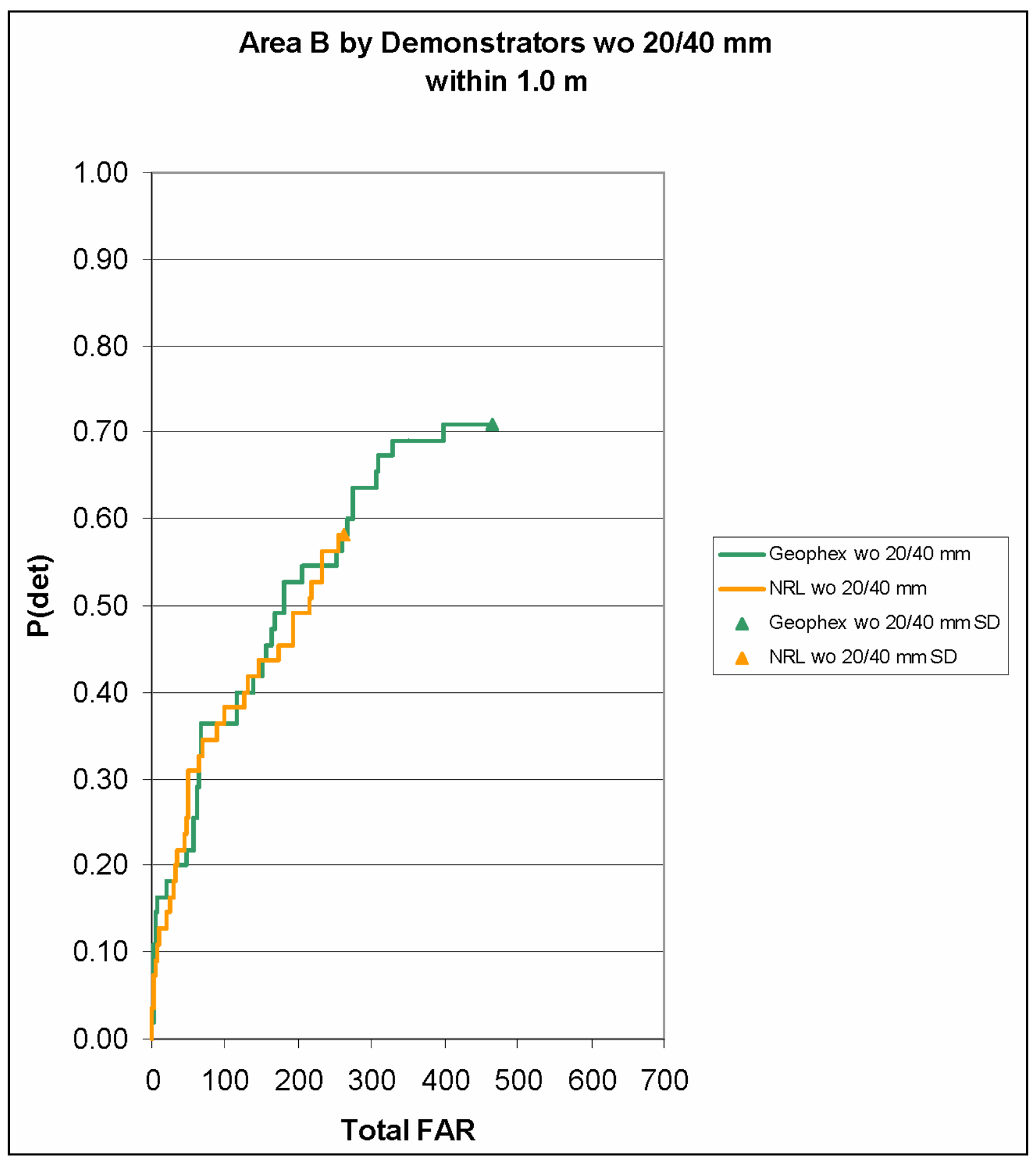

Figure $C 10$. Area $B-P($ det $)$ vs. total $F A R$ within $1.0 \mathrm{~m}$ for demonstrators wo $20 / 40 \mathrm{~mm}$ 


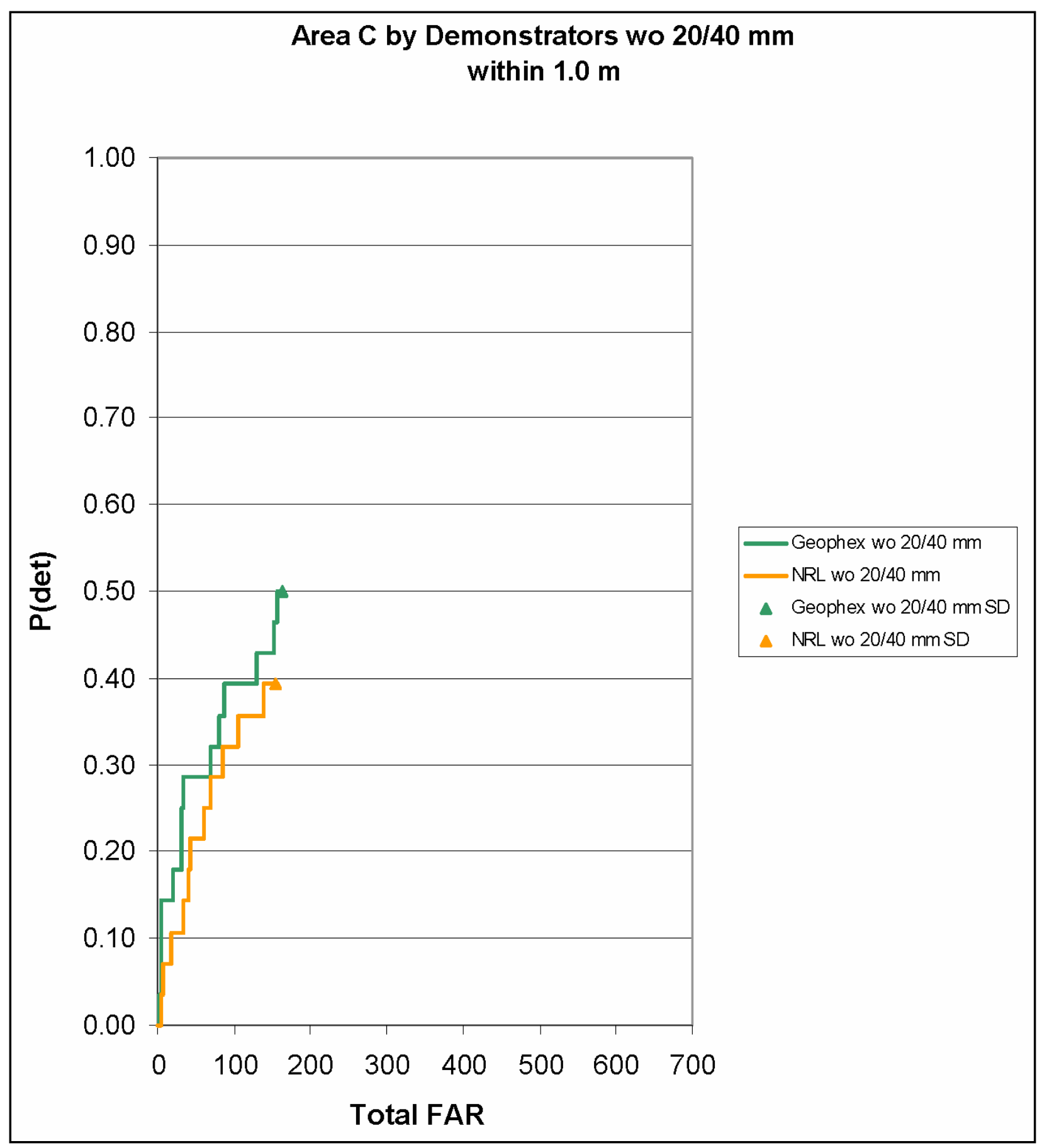

Figure $C 11$. Area $C-P($ det) vs. total FAR within $1.0 \mathrm{~m}$ for demonstrators wo $20 / 40 \mathrm{~mm}$ 


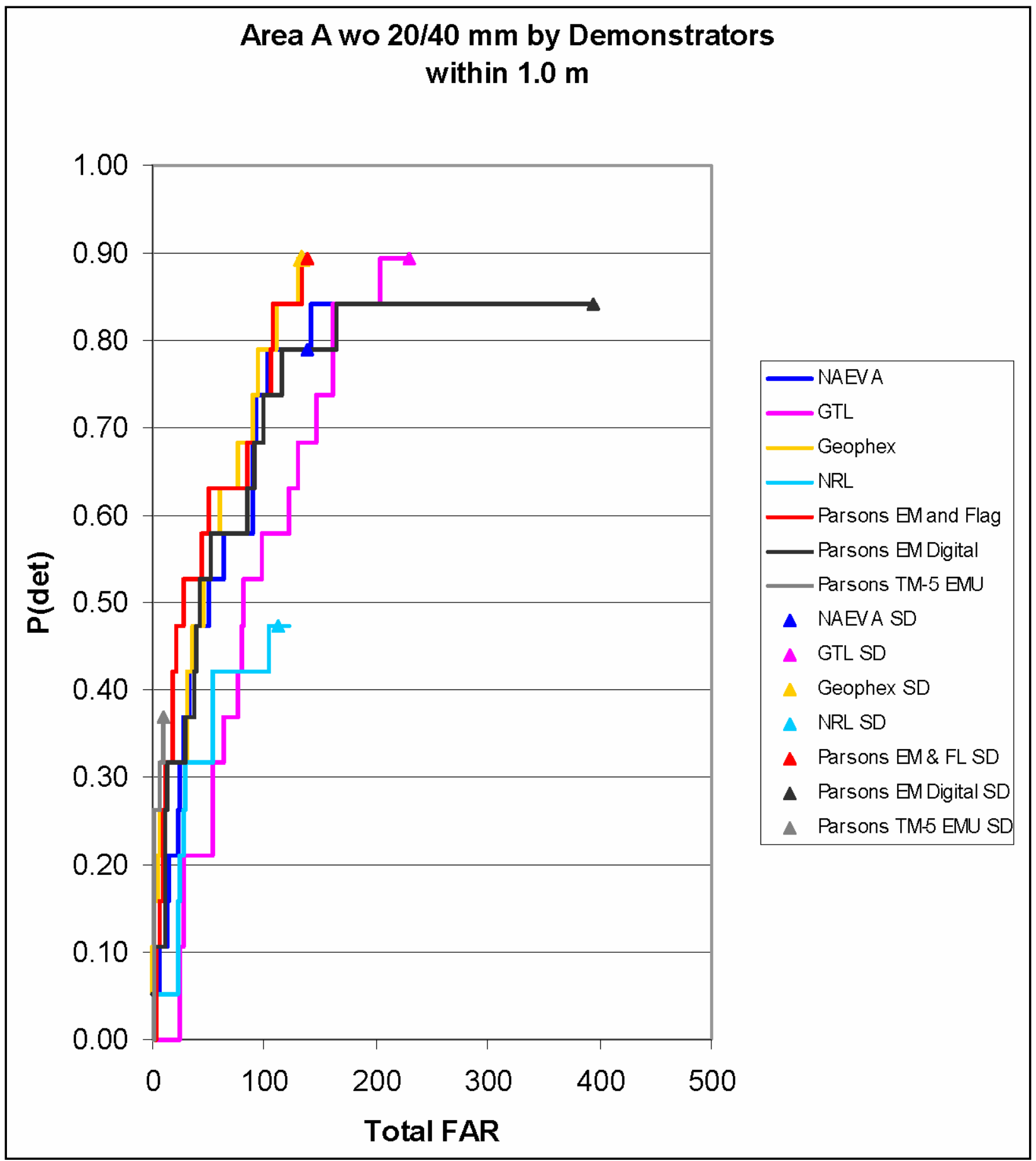

Figure C12. Area A wo $20 / 40 \mathrm{~mm}-\mathrm{P}$ (det) vs. total FAR within $1.0 \mathrm{~m}$ for all demonstrators 


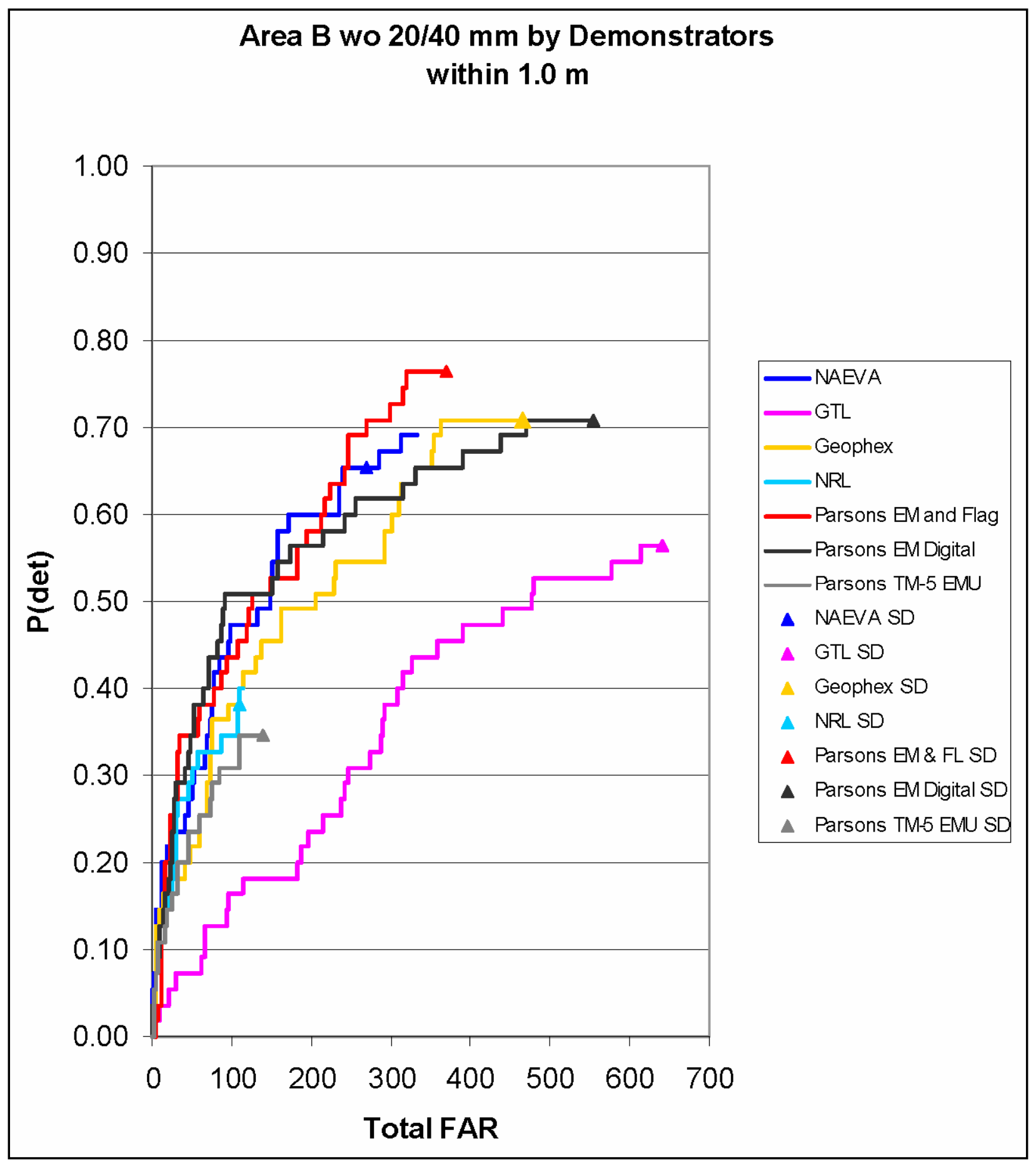

Figure $\mathrm{C} 13$. Area $\mathrm{B}$ wo $20 / 40 \mathrm{~mm}-\mathrm{P}$ (det) vs. total $\mathrm{FAR}$ within $1.0 \mathrm{~m}$ for all demonstrators 


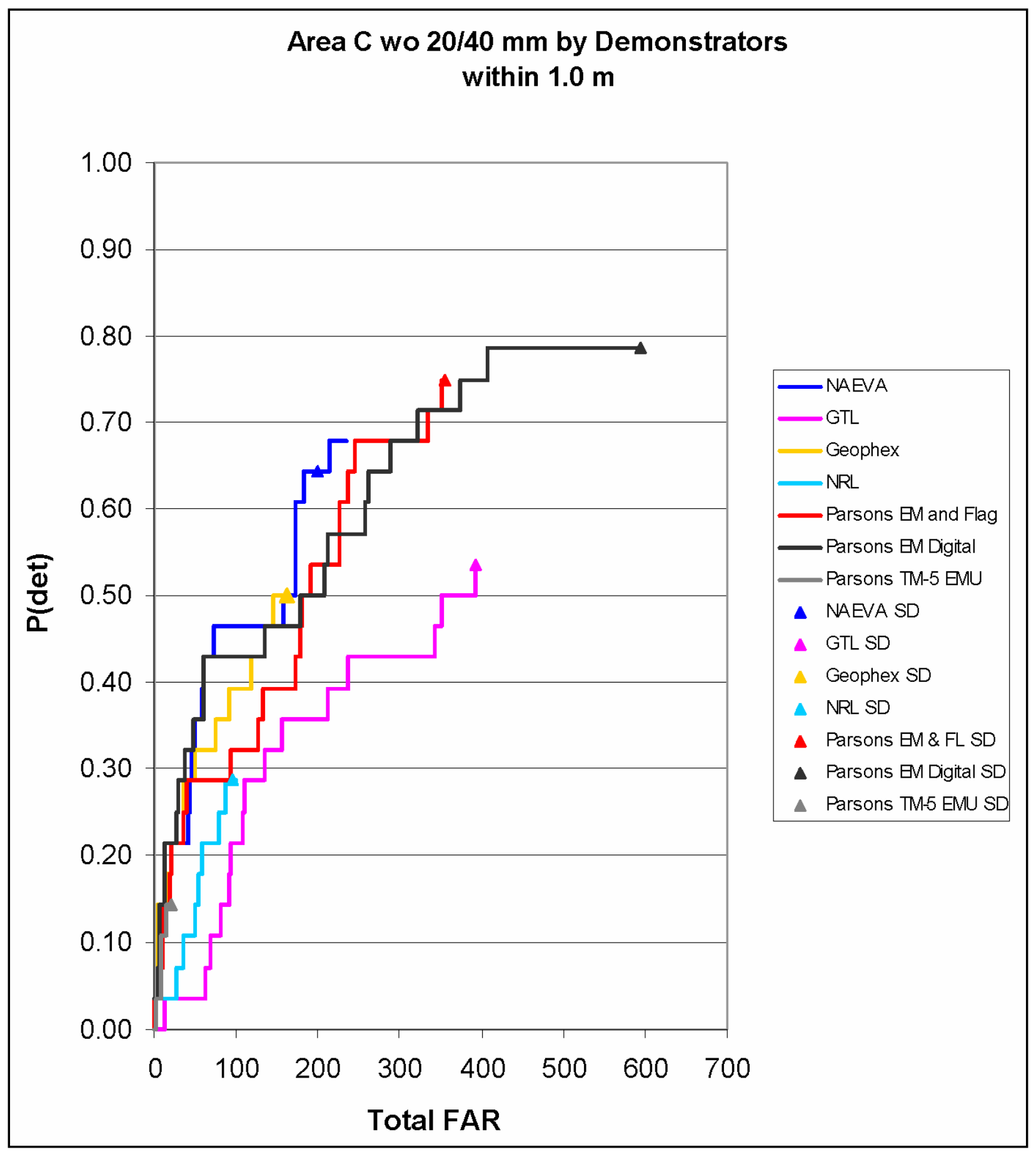

Figure C14. Area $\mathrm{C}$ wo $20 / 40 \mathrm{~mm}-\mathrm{P}$ (det) vs. total FAR within $1.0 \mathrm{~m}$ for all demonstrators 


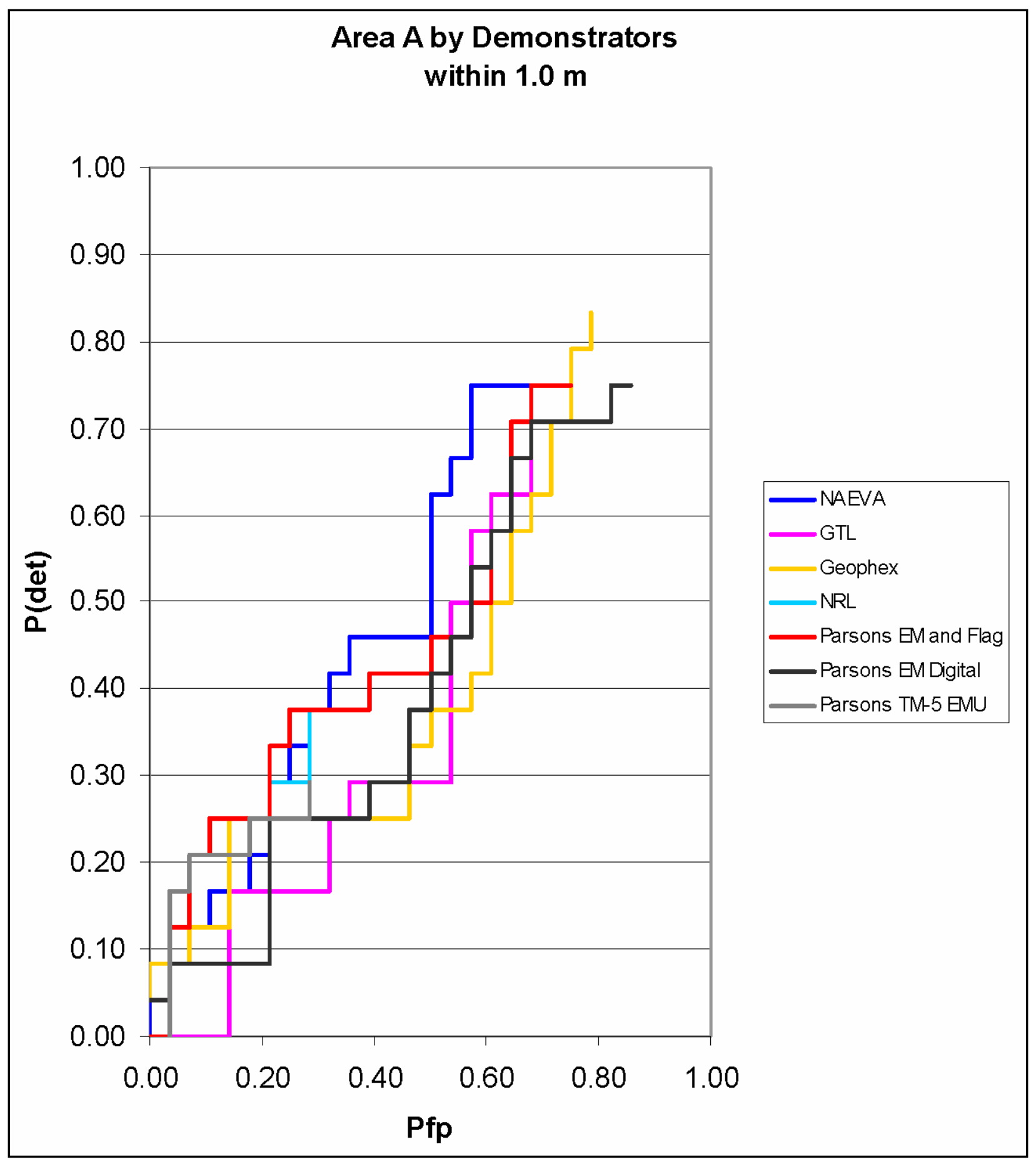

Figure $\mathrm{C} 15$. Area $\mathrm{A}-\mathrm{P}$ (det) vs. Pfp within $1.0 \mathrm{~m}$ for all demonstrators 


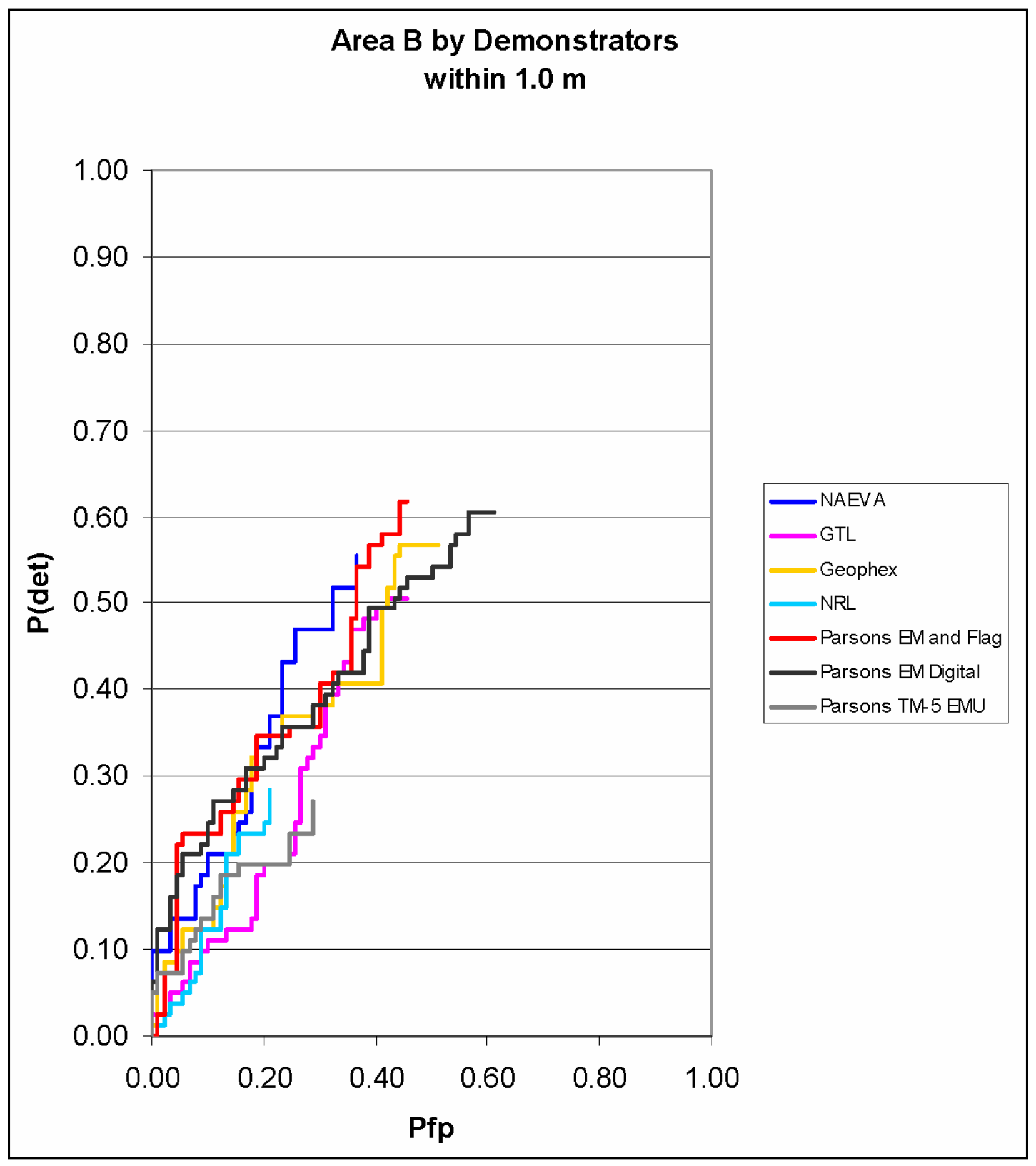

Figure $\mathrm{C} 16$. Area $\mathrm{B}-\mathrm{P}$ (det) vs. Pfp within $1.0 \mathrm{~m}$ for all demonstrators 


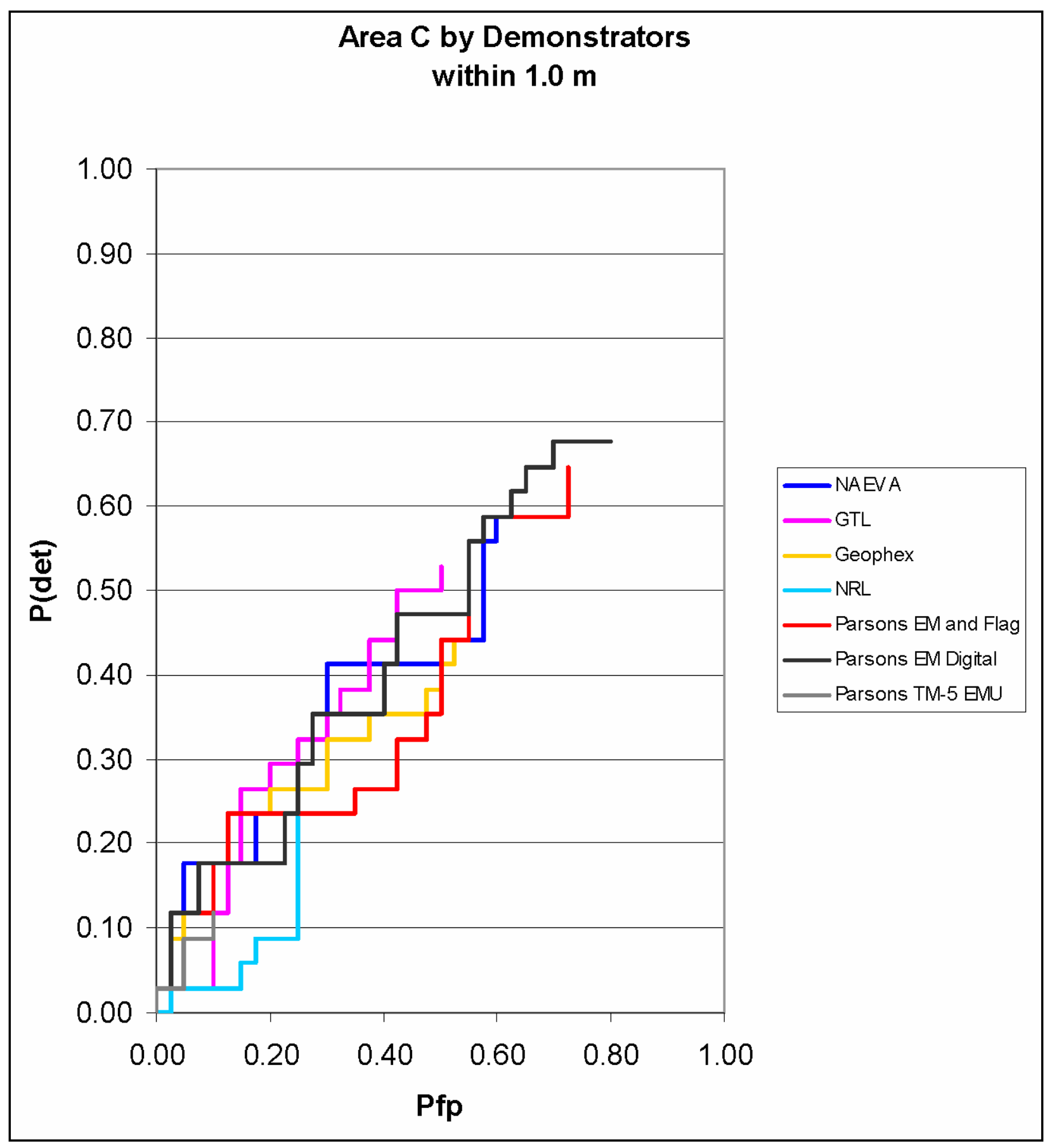

Figure $\mathrm{C} 17$. Area $\mathrm{C}-\mathrm{P}(\mathrm{det}) \mathrm{vs.}$ Pfp within $1.0 \mathrm{~m}$ for all demonstrators 
Figures $\mathrm{C} 18$ through $\mathrm{C} 20$ show the systems' ability to discriminate ordnance from nonordnance (both geologic and metallic) based solely on signal strength. The ROC curves for all demonstrators tend to fall within a narrow band and support no conclusions regarding the various systems' discrimination capability.

\section{Overall best performances}

Overall, the best performances were achieved in Area A where NAEVA, GTL, Geophex, Parson EM and flag, and Parson EM-61 digital had a maximum achievable $\mathrm{P}(\mathrm{det})$ of at least 75 percent when including detections within $1.0 \mathrm{~m}$. However, none of the demonstrators reached the $\mathrm{P}(\mathrm{det})$ required for Kaho'olawe Tier II clearance (85 percent). The $\mathrm{P}(\mathrm{det})$ in Areas B and C were significantly lower for all demonstrators. 


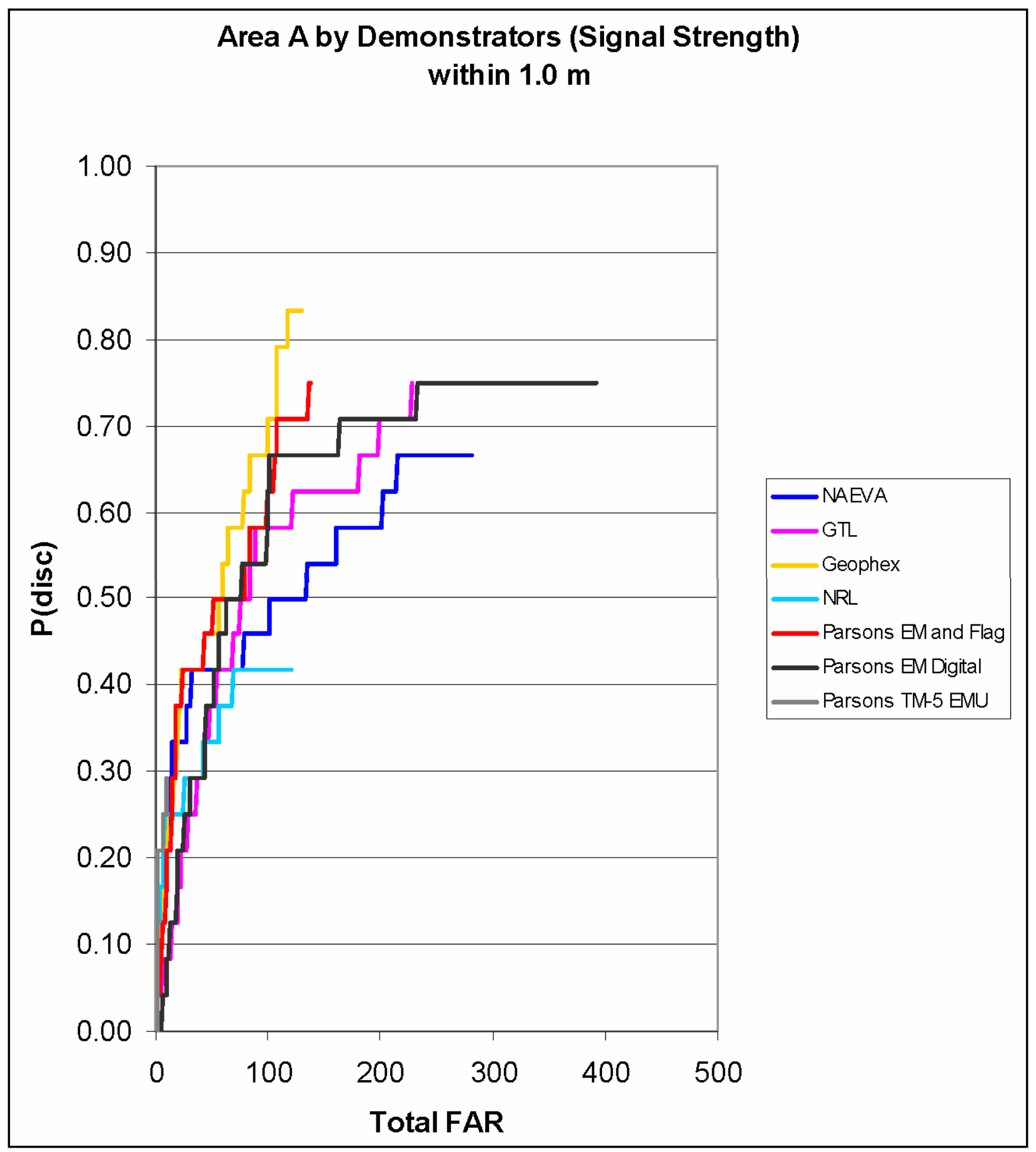

Figure $C 18$. Area $A-P($ disc) (prioritized by signal strength) vs. total $F A R$ within $1.0 \mathrm{~m}$ for all demonstrators 


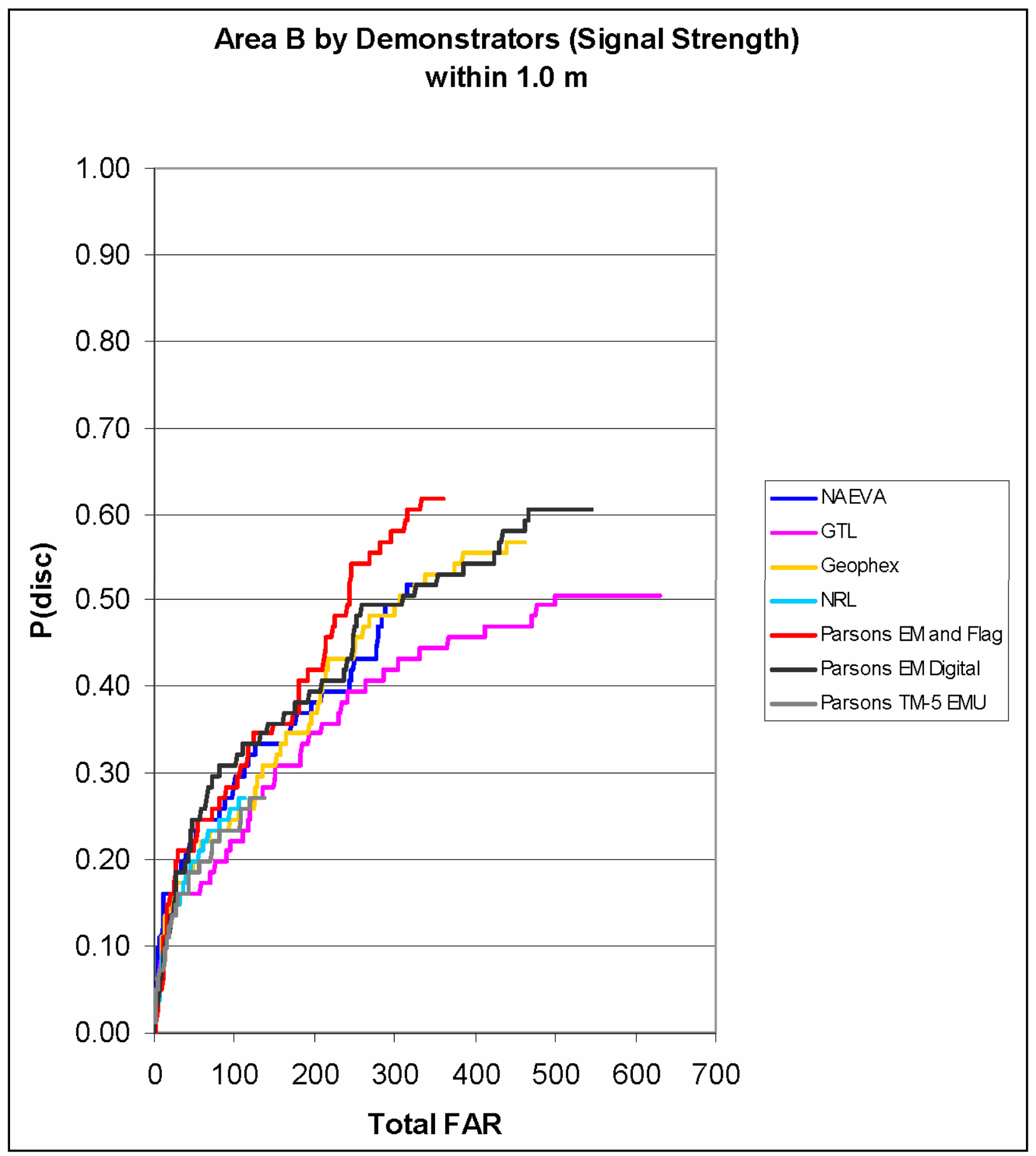

Figure $C 19$. Area $B-P($ disc) (prioritized by signal strength) vs. total FAR within $1.0 \mathrm{~m}$ for all demonstrators 


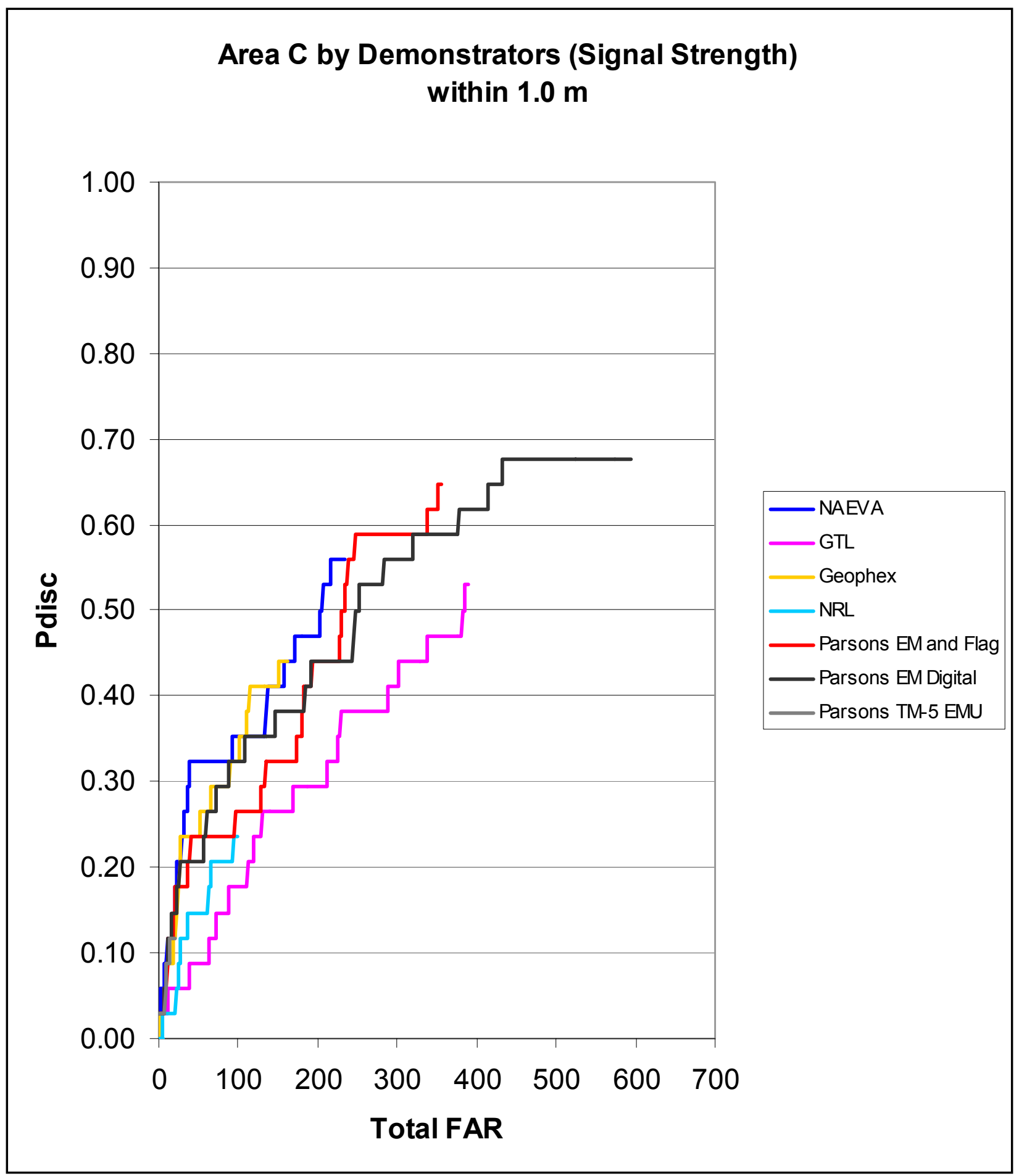

Figure $\mathrm{C} 20$. Area $\mathrm{C}-\mathrm{Pdisc}$ (prioritized by signal strength) vs. total FAR within $1.0 \mathrm{~m}$ for all demonstrators 


\section{Appendix D Mean Location Error Analysis}

This Appendix contains the scatter graphs of the mean location error in northing and easting for each emplaced item, grouped by areas, shown in Figures D1 through D3. Also, scatter graphs of the mean location error in northing and easting for each demonstrator are given for each area in Figures D4 through D6. The graphs indicate sufficient grouping of the targets within $0.5 \mathrm{~m}$ of their placement versus their reported detection location by the demonstrators. Item $2 \mathrm{C} 623$ was the only emplaced item that was an ordnance, having at least three demonstrators with the same positioning error over $1.0 \mathrm{~m}$. The depth of this item was $0.7 \mathrm{~m}$. The location error probably resulted from the detection of nonemplaced clutter.

The following emplaced items, targets 2E 640, 1D 542, 1D 554, and 1D 550, had a mean location error over $1.0 \mathrm{~m}$. Of these targets, 1D 550 was the only one that had three or more demonstrators with the same positioning error.

The following emplaced items, targets 3E 711, 5D 841, 4C 761, 5C 821, 3E 718 , 3E 715, 5B 793, 4E 777, 5D 842, 5C 823, 3D 97, and 4C 751, had a mean location error over $1.0 \mathrm{~m}$. Of these targets, $4 \mathrm{C} 751$ was the only one that had three or more demonstrators with the same positioning error.

The following emplaced items, 1B 163, 2C 611, 1C 530, 2C 623, 2B 596, 2B 603 , and $2 \mathrm{~A} 581$, had a mean location error over $1.0 \mathrm{~m}$. Of these targets, $2 \mathrm{C} 623$ was the only one that had three or more demonstrators with the same positioning error. 


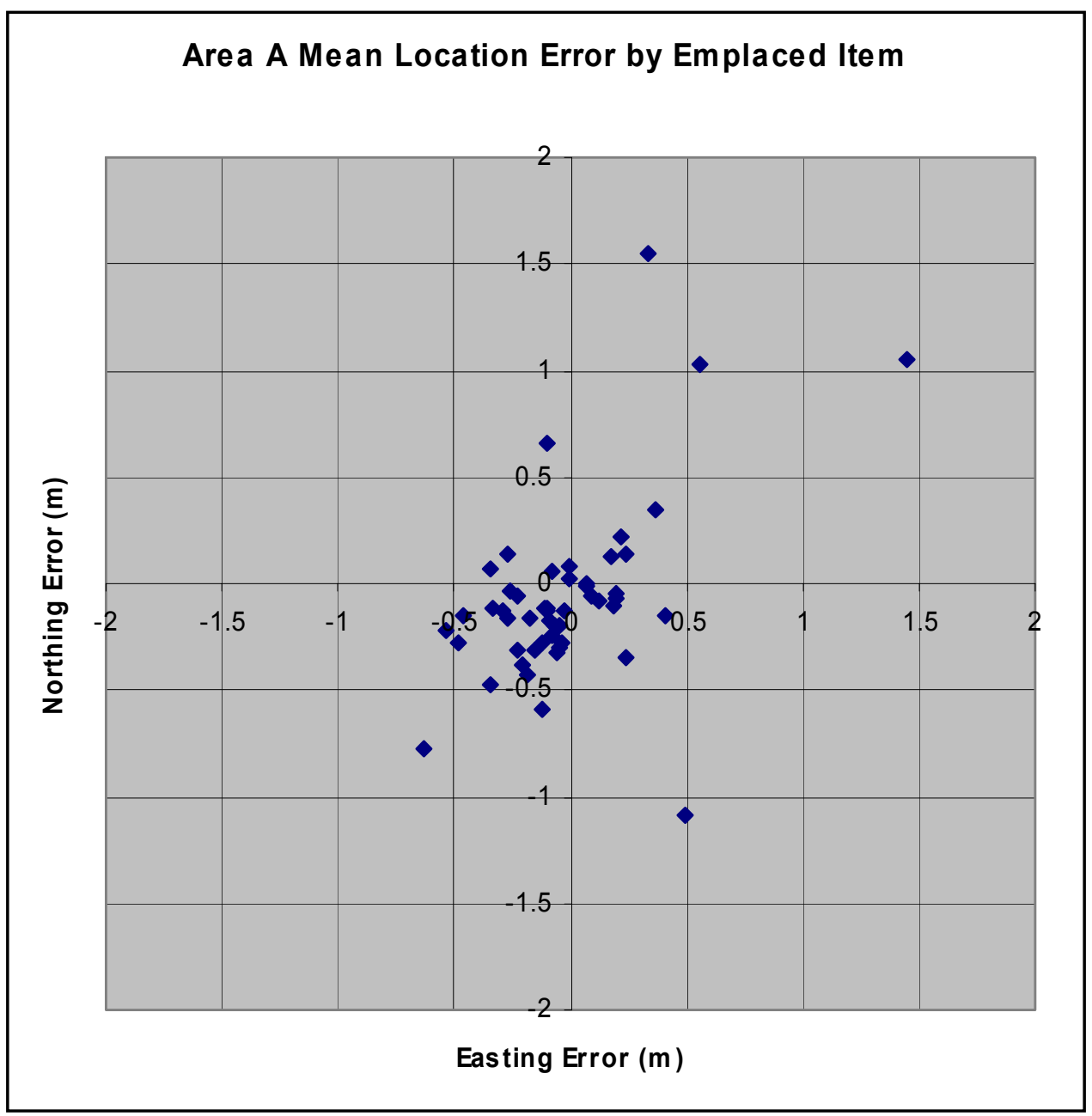

Figure D1. Area A - Mean location error by emplaced item 


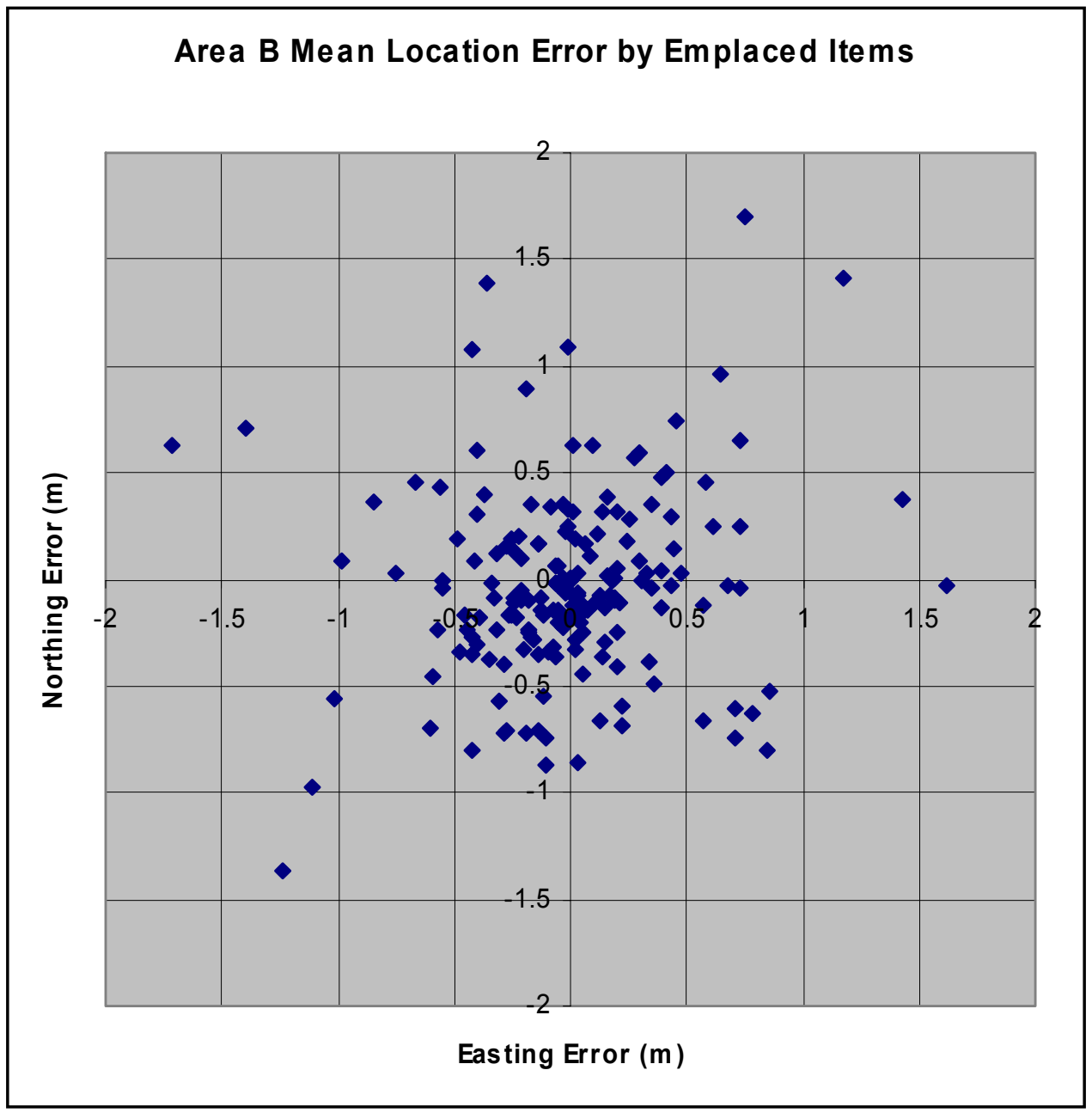

Figure D2. Area B - Mean location error by emplaced item 


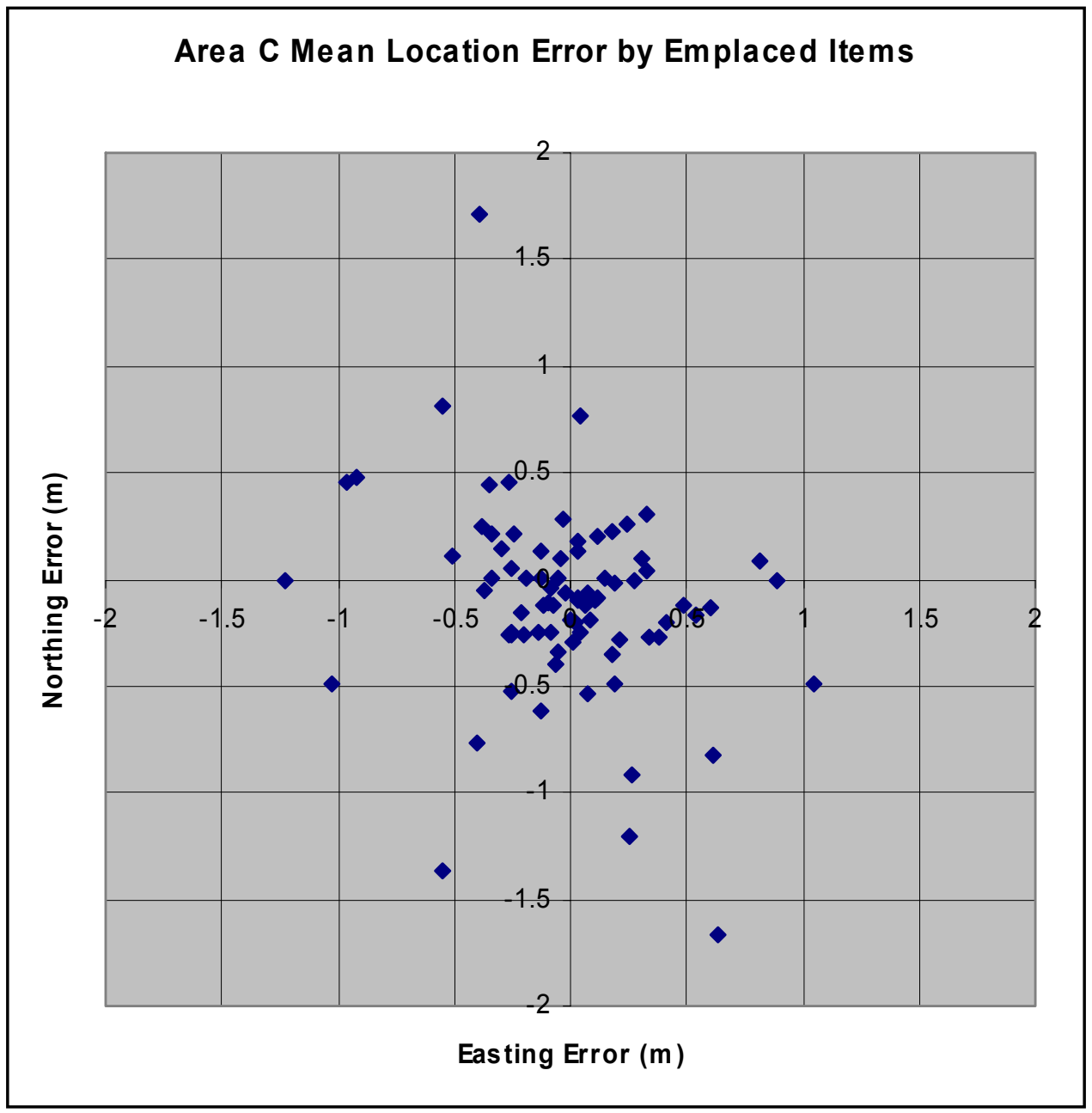

Figure D3. Area C - Mean location error by emplaced item 


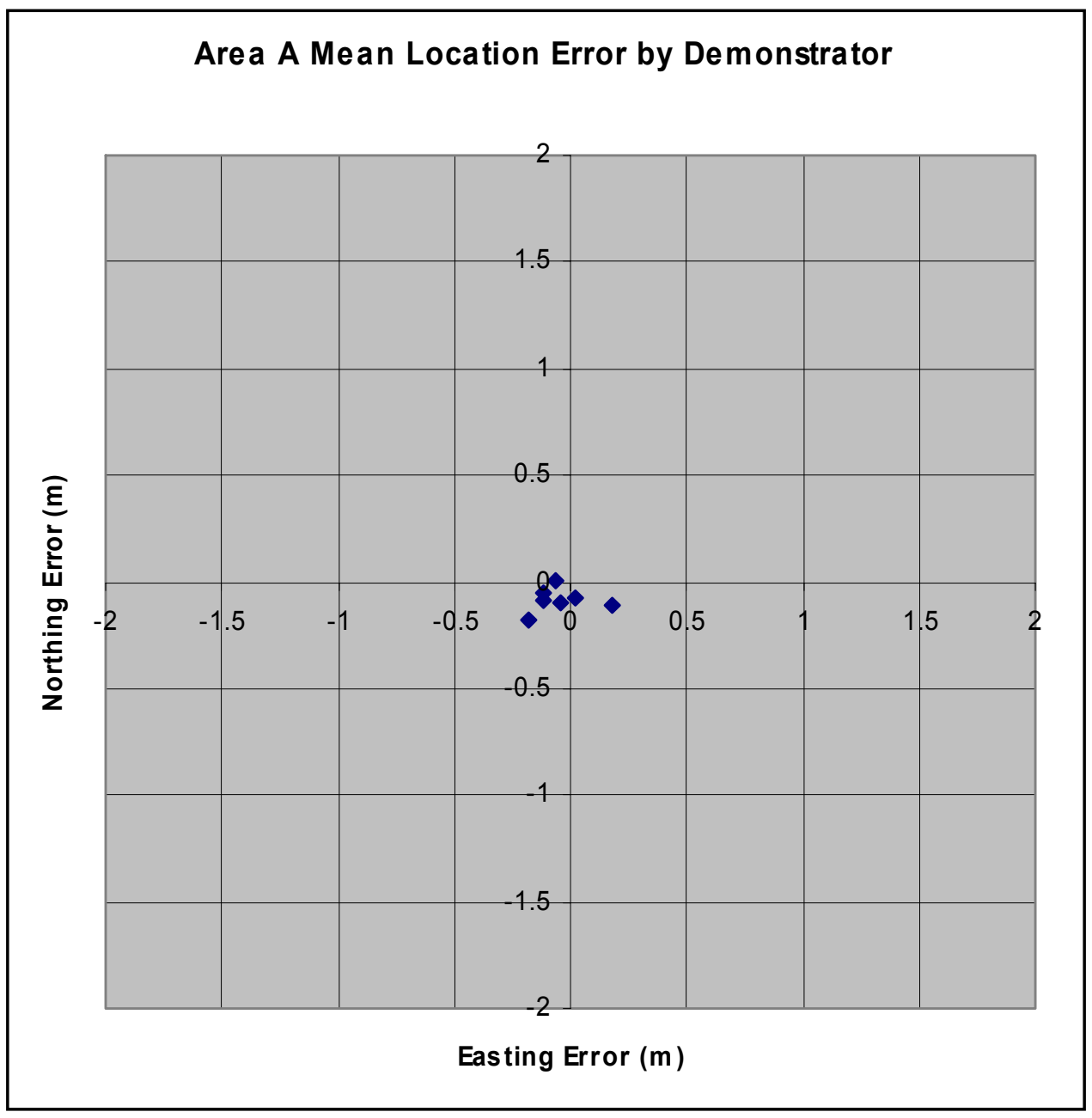

Figure D4. Area A - Mean location error by demonstrator 


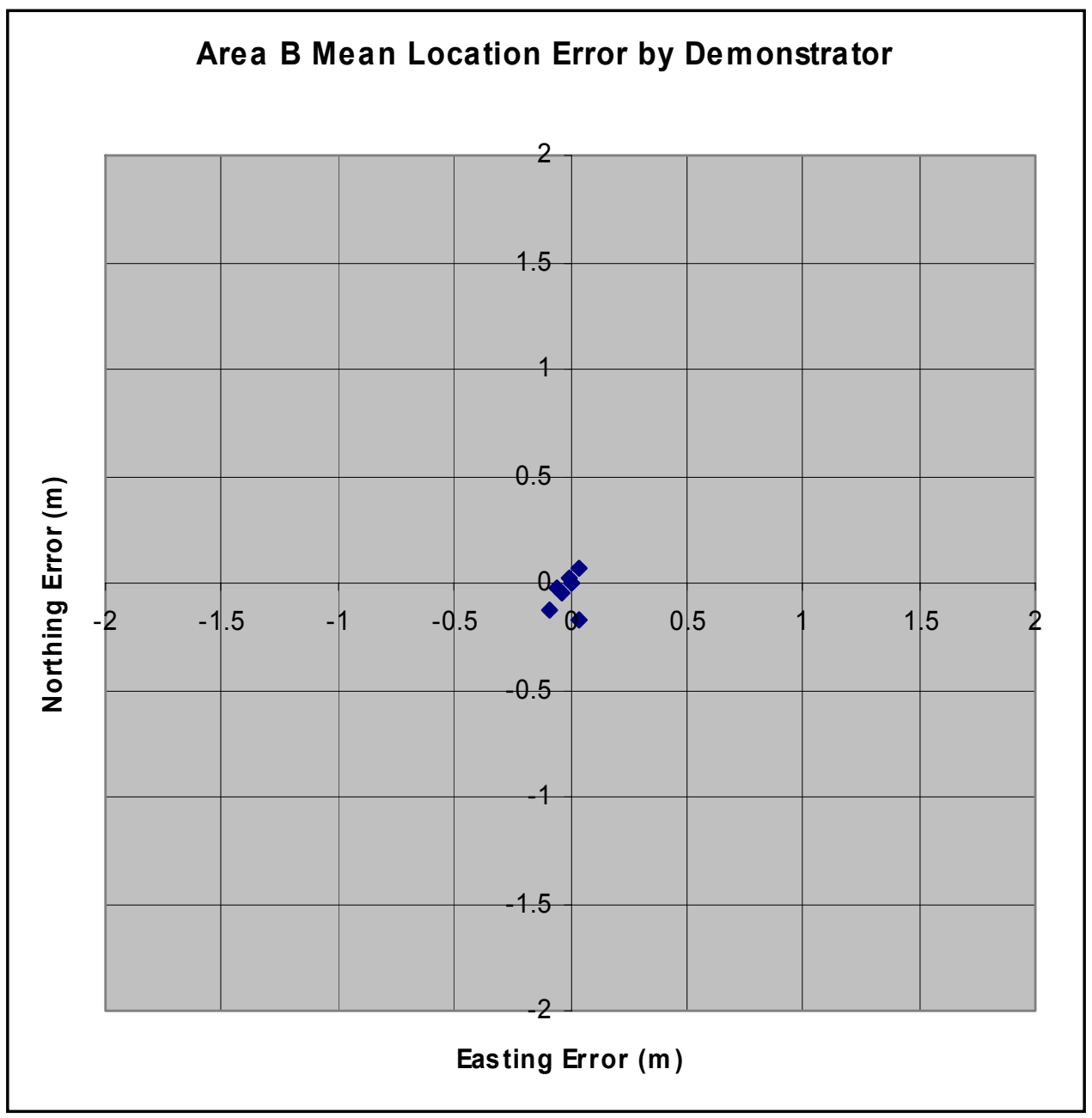

Figure D5. Area B - Mean location error by demonstrator 


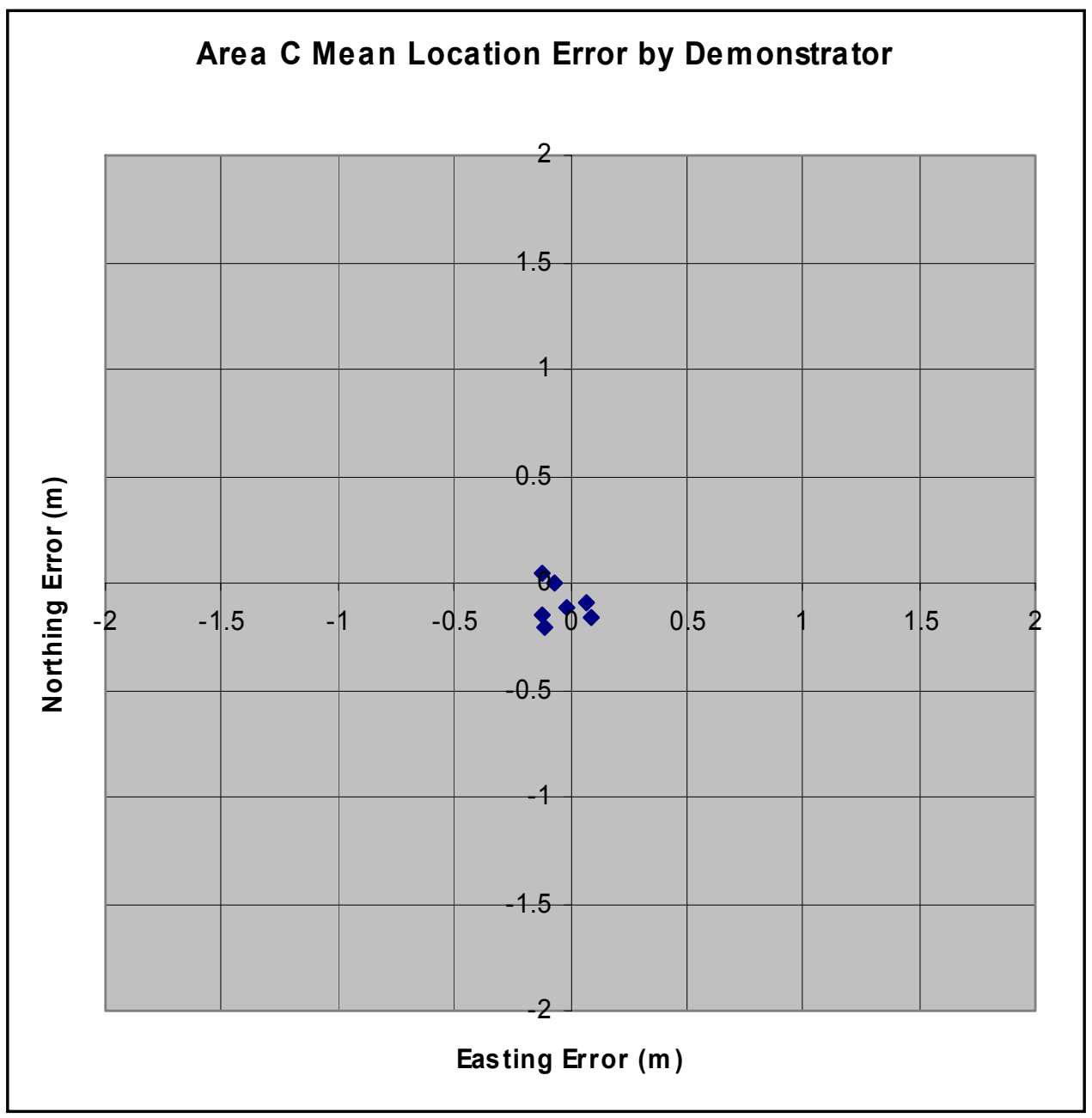

Figure D6. Area C - Mean location error by demonstrator 


\section{Appendix E Demonstrators' Data Collection and Analysis Plans}

This Appendix contains the unedited documents submitted by each of the demonstrators as they describe their planned or actual methodologies for performing the field surveys and analyze the data as part of the Kaho'olawe demonstrations. Note that Geophysical Technology Limited (GTL) did not submit the required documents. 


\title{
E.1. NAEVA Geophysics Inc.
}

\author{
Survey, Quality Control and Data Processing \\ Methodology \\ Advanced UXO Discrimination/Discrimination \\ Technology Demonstration \\ Kaho'olawe Island, Hawaii
}

\author{
NAEVA Geophysics, Inc. \\ North American Exploration of Virginia, Inc.
}

\section{November 2001}

Introduction, NAEVA Kaho'olawe Procedures

After 14 days of testing, calibration, and algorithm development (for basalt response discrimination) on Kaho'olawe (September 17 -30, 2001), North American Exploration of Virginia, Inc. (NAEVA) (with Geophysical Associates (GPA)), demonstrated at Kaho'olawe during the 4-day period October 1-4, 2001. The overall setup for the exercise is described in the Advanced UXO Discrimination/Discrimination Technology Demonstration Plan (31 Aug. 2001) by the Naval Explosive Ordnance Disposal Technology Division (NAVEODTECHDIV). Multiple samples of 20 to 30 inert ordnance types and a variety of nonordnance and clutter items were emplaced on each of a 1-hectare test grid and ten $30-\times 30-\mathrm{m}$ test grids. The schedule and budget allowed approximately 2 days per hectare.

Measurements were also made on a small self-evaluation test grid and in a small trench. NAEVA personnel respected all issues and procedures in the Site Health and Safety Plan.

\section{4-Day Field Test, Calibration, and Revision-Optimization Period}

Objectives during the test, calibration, and revision-optimization period were: (a) evaluation of new compensation coil and other noise reduction measures, and (b) measurement and evaluation of the spatially variable background response of basalt and basaltic soils in various locations.

To these ends, NAEVA made repeated measurements over basaltic materials on the test grids (with and without metallic targets) at various speeds (including static), with and without the compensation coil, at both medium- and high-pulse repetition rates, and with careful zero calibration procedures to minimize instrument drift. The main objective during the revision - optimization period was to determine whether the high background response of basaltic materials could be removed (by auto-leveling) and/or incorporated into the chi-squared fitting 
procedures (by use of a characteristic basalt decay vector). The latter proved more feasible.

Background response of basalt was spatially variable on the test grid. Figure E1.1 displays stacked profiles from a single 10-m lane (test grid lane $0-10$ ), with highly variable basalt background response. Figure E1.2 shows typical basalt amplitude response decay (log-linear).

Field and bench tests revealed that the NAEVA EM-63 instrument had satisfactory instrument noise in the medium frequency (26 time gates) mode. However, the instrument drift rate was excessive, especially in the early gates ( 10 to $20 \mathrm{mV}$, generally negative, during the first 5 to $10 \mathrm{~min}$ of operation, decreasing thereafter).

Parsons and the U.S. Army Engineer Research and Development Center (ERDC) very kindly made their EM-63 data acquisition (DAQ) consoles available for comparative tests. It was determined that these consoles drifted less ( 5 to $10 \mathrm{mV}$ over 5 to $10 \mathrm{~min}$ ) but were much noisier in all time gates. Figure E1.3 compares drift and noise for these three EM-63 consoles. We therefore decided to continue to use the NAEVA rental console.

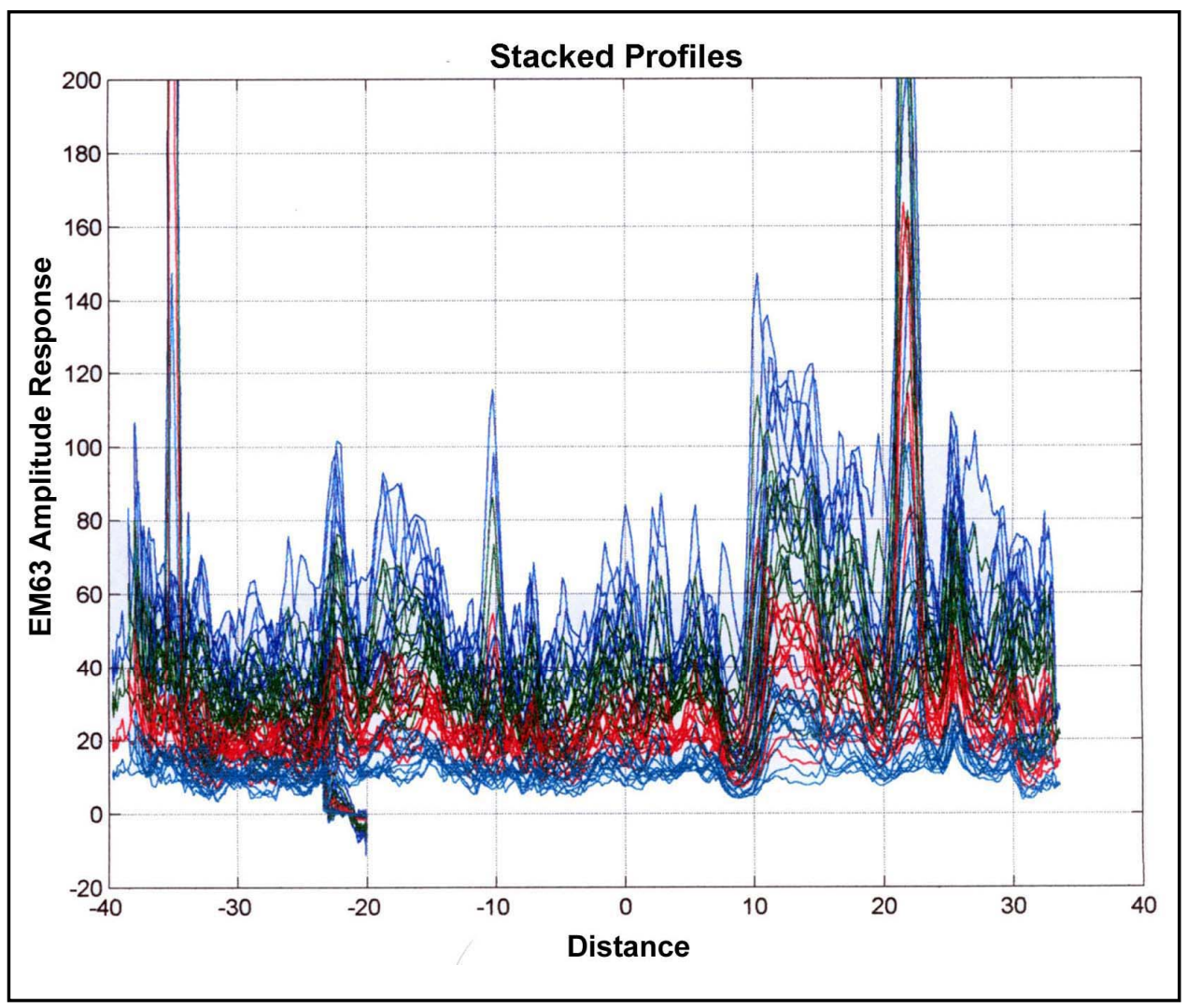

Figure E1.1. Stacked profiles of test grid lane 0-gates 1:3:10 


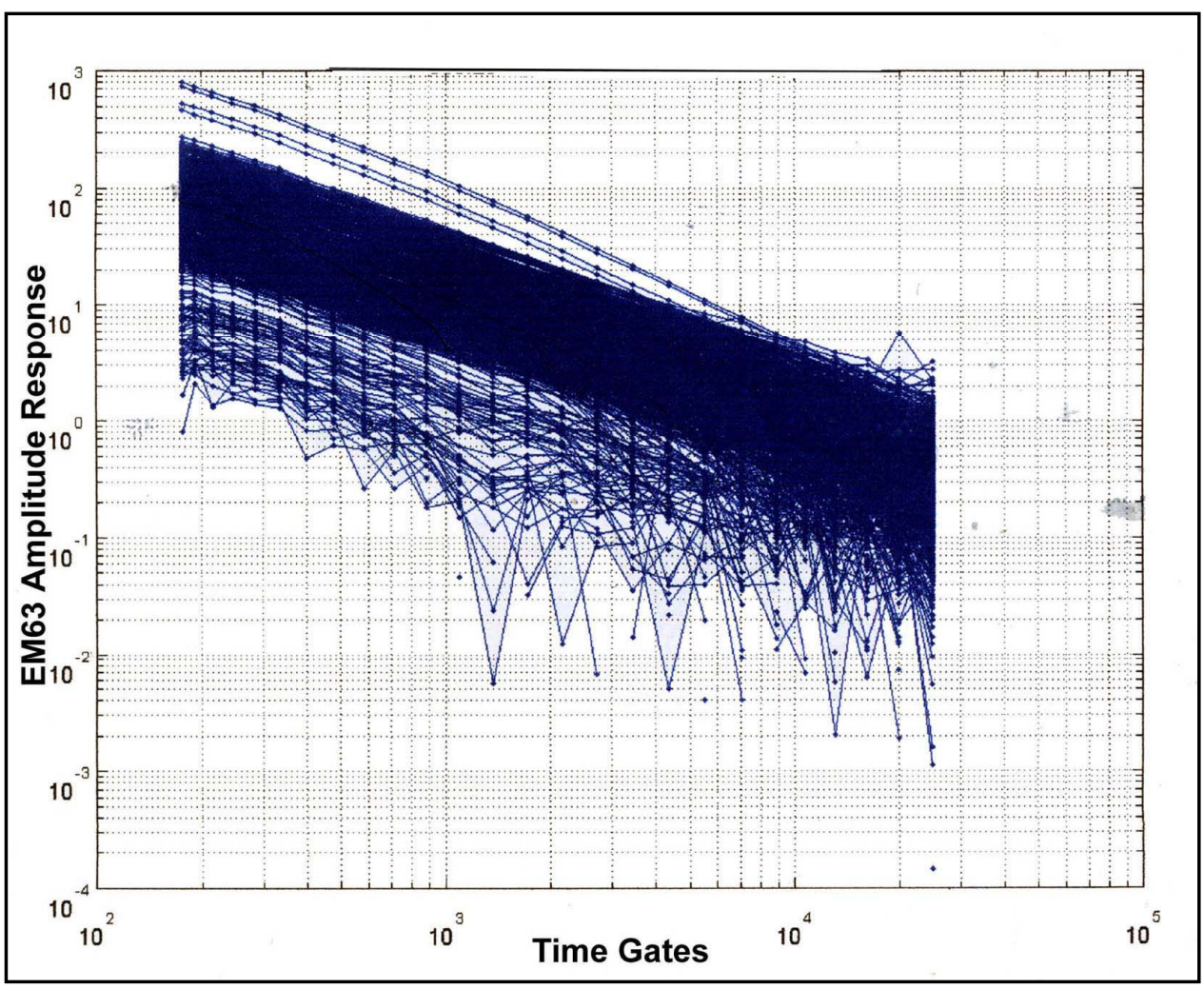

Figure E1.2. Basalt EM-63 Amplitude Response Decay ( 1/T)

It was therefore necessary to stabilize (warm up) the EM-63 on the test bench for as long as possible (10 min or so), and then rezero, before surveying each $10-\mathrm{m}$ lane. Then it was necessary to place the instrument on the test bench again in order to determine the amount of drift during the survey. Unfortunately, the drift was still substantial and nonlinear. This necessitated special steps in postprocessing to remove drift effects and variable basalt background response.

\section{Specific Demonstration Survey Plan (Data Acquisition)}

The EM-63 was operated on a nonmetallic test table in static mode for approximately $10 \mathrm{~min}$ at the beginning and end of each lane file to zero the instrument (away from possible background response) before each survey period and check for calibration drift after each survey period. Figure E1.4 illustrates EM-63 zeroing in air before grid lane surveying. A standard 8.89-cm (3.5-in.) iron calibration sphere was placed at zero depth, just north of the north end of the first survey line in each lane, to verify stable amplitude response. The initial line in each lane file was surveyed in southeast and northwest directions to verify data repeatability and satisfactory positional latency (lag) corrections.

EM-63 data were acquired on all three demonstration grids. Data were measured in narrow blocks or lanes, approximately 10-m wide, over the full northwest-southeast extent of each grid (or contiguous group of grids). This was done because of memory limitations in the EM-63 and to avoid longer-term zero 

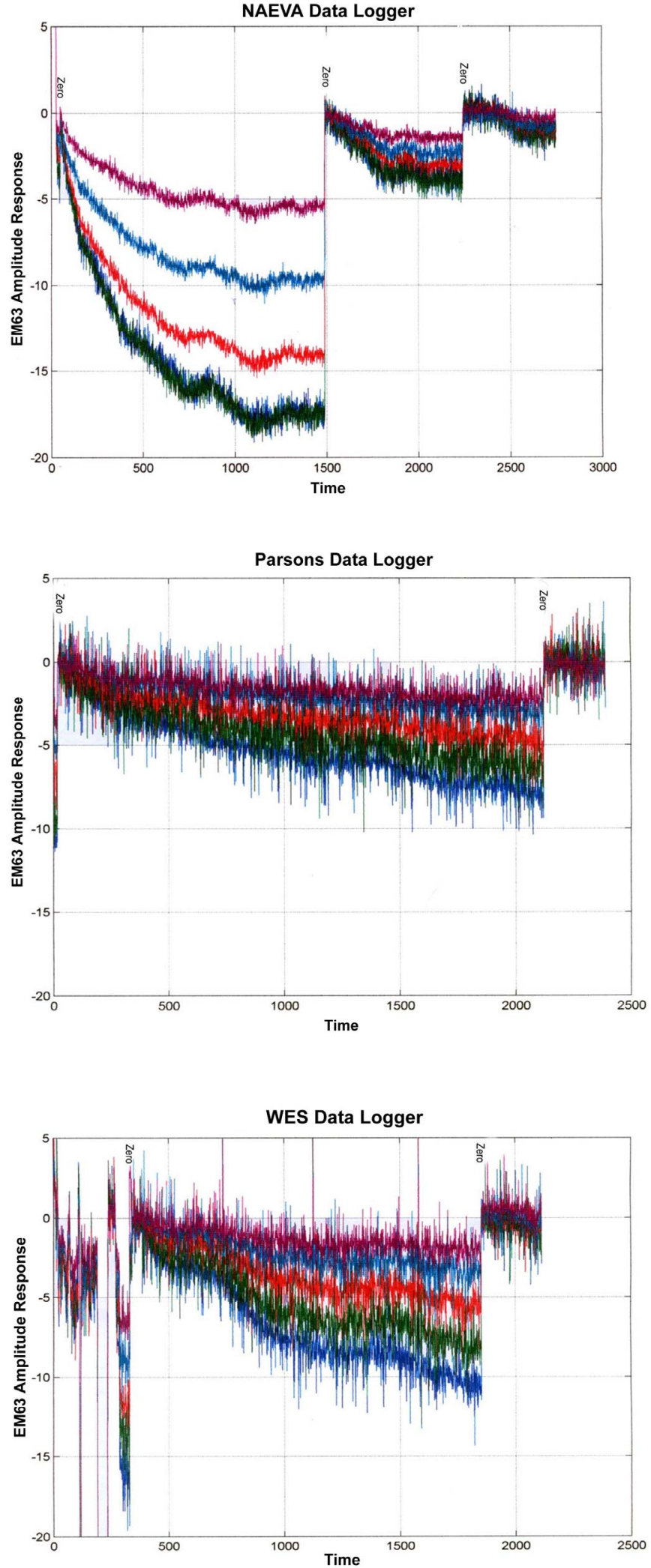

Figure E1.3. Console comparison of drift 


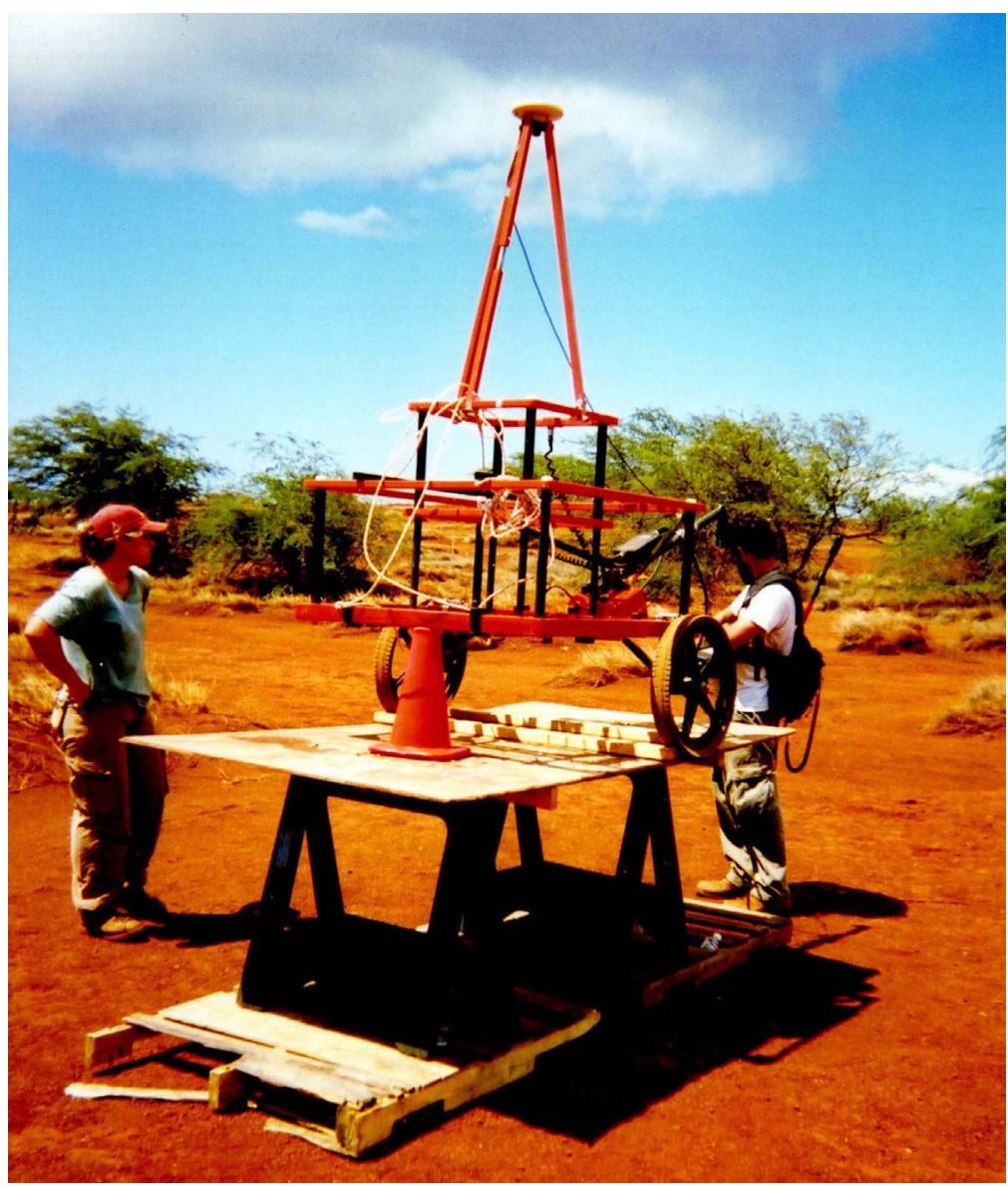

Figure E1.4. Zeroing of the EM-63 before surveying

calibration drift (data acquisition required approximately 30 to $60 \mathrm{~min}$ per $10-\mathrm{m}$ lane). Figure E1.5 illustrates surveying with the EM-63. Northeast-southwest guide ropes (parallel to prevailing wind) were spaced $2 \mathrm{~m}$ apart to ensure straight survey lines with a $0.5-\mathrm{m}$ line spacing.

In accordance with instructions, the demonstration grids were surveyed in three blocks, which were designated A (the northernmost four 30- $\times 30$-m grids), $\mathrm{B}$ (the 1-hectare grid), and C (the last six 30- $\times 30$-m grids), as shown in Figure E1.6. Each lane was numbered in order from southeast to northwest (A0-10, A10-20, A20-30, etc. for example). Each raw (binary EM-63-GPS composite) lane file contains approximately 1 to $2 \mathrm{Mb}$ of data. When necessitated by GPS or other data problems, repeat lane files were measured, and named A10-20b, etc. (This paper (E.1) was written and submitted by NAEVA. This was their recordkeeping method, A10-20, and the repeat was A10-20b.) GPS positions were acquired at a rate of one per second, and EM-63 readings were collected at a rate of five per second, yielding a data density of one reading approximately every 10 to $20 \mathrm{~cm}$. 


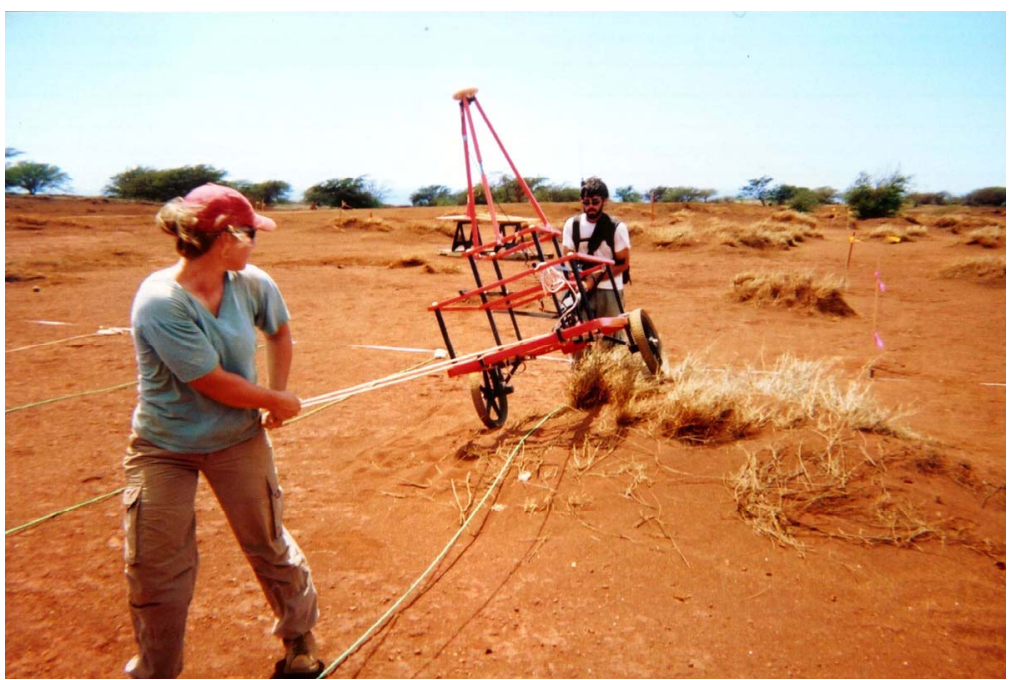

Figure E1.5. Traversing Kaho'olawe Demonstration Grid B with the EM-63 (Ropes at 2-m intervals)

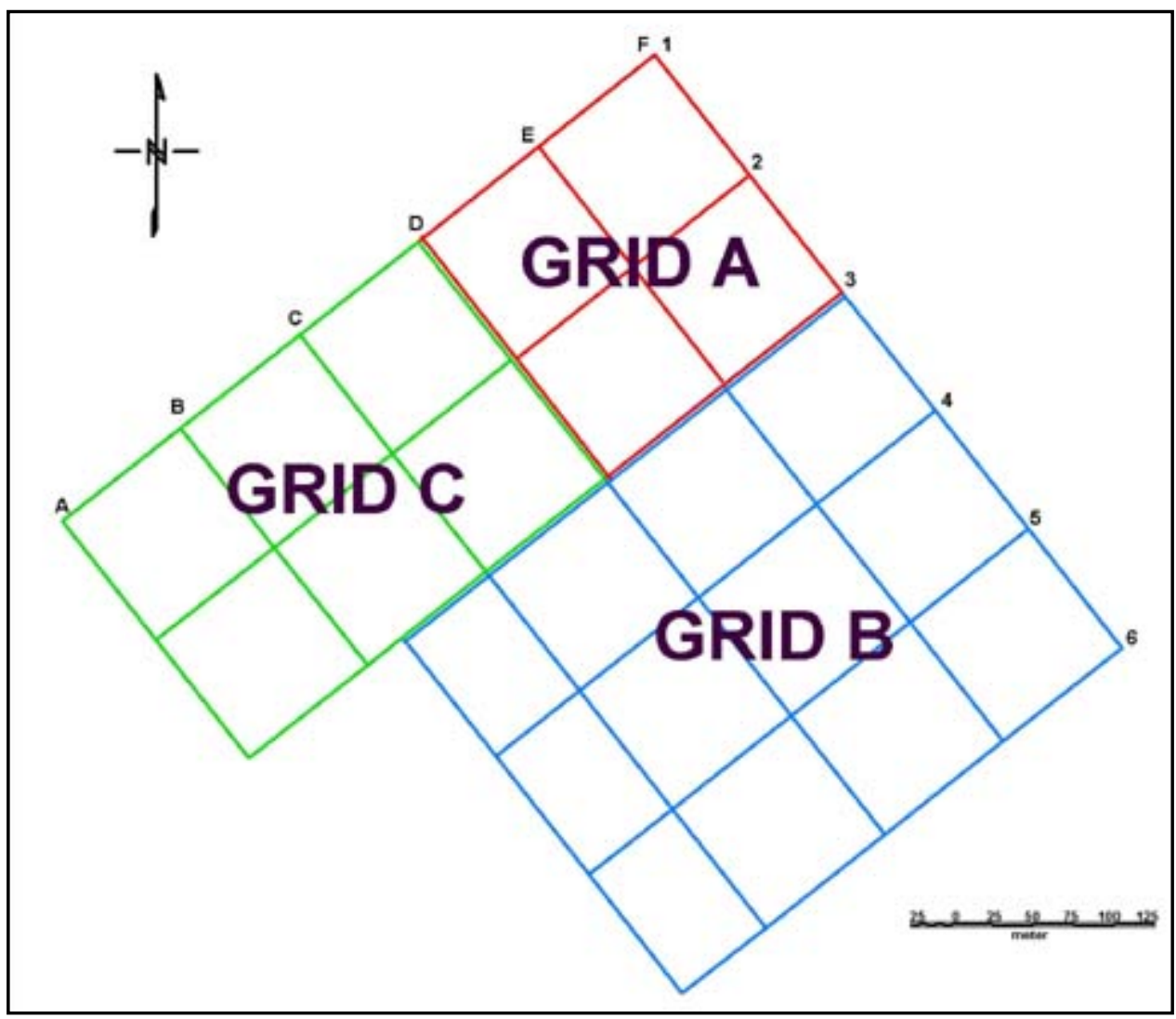

Figure E1.6. Site map of demonstration area 


\section{Additional Decay Curve Calibration Measurements}

Additional (different) inert ordnance samples were made available at the Kaho'olawe demonstration site. Bench test decay curve measurements were made on these additional items during the period September 17 through 27, 2001, as time permitted. Figure E1.7 illustrates additional ordnance tested at Kaho'olawe.
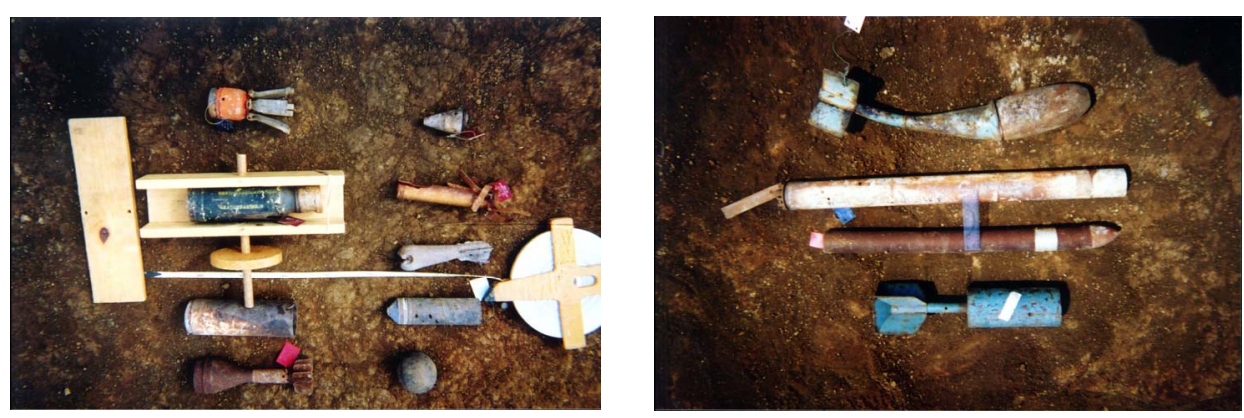

Figure E1.7. Ordnance samples available on Kaho'olawe

\section{NAEVA Data Quality Control Plans}

As previously mentioned, the EM-63 was static tested for zero calibration and instrument (plus ambient) noise at the beginning of each survey lane file. The first line was repeated (bidirectional) to verify amplitude and location repeatability. As soon as the file was complete, it was checked for data gaps and/or poor GPS position recovery, and portions were repeated if necessary (generally, resulting from poor satellite availability).

The repeatability of the first line in each grid lane file (and the amplitude response of the calibration sphere) was also verified, and terrain noise was inspected. Spatially variable background response was present in the early time gates at the Kaho'olawe grids and was removed from the field data (or compensated) before target decay curves could be compared.

Figure E1.8 shows GPS position checks for a problematic lane file (Lane B18-28, tree at northeast end); black denotes GPS first quality "fix," while red denotes GPS second quality "float." "Float" positions were sometimes, but not always, usable.

\section{Data Processing}

The basic EM-63 data processing and analysis steps (as practiced at the Jefferson Proving Ground "Five" demonstration in year 2000) are as follows:

a. GPS checks: GPS position integration (interpolation, latency corrections). 


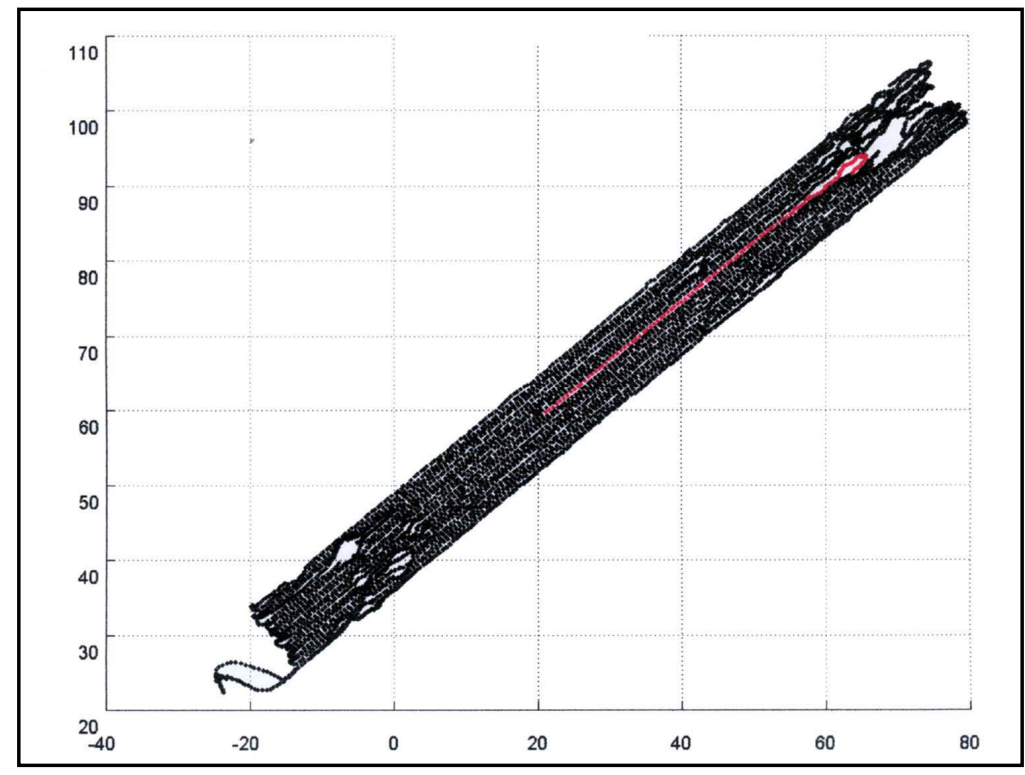

Figure E1.8. Check Ashtech GPS positions, Lane B18-28

$b$. Auto-Leveling (all gates): Remove decaying background response and calibration drift across all time gates. (Note, this was not possible at Kaho'olawe.)

c. Visual Inspection (profiles and plan contour maps) and Editing: Remove bad data points, recognize data gaps, cut outside the grid, and split lines for GEOSOFT. Repeat data acquisition (DAQ) if necessary, cropping to $0.5 \mathrm{~m}$ outside the grid boundaries.

d. Target Picking: Select all targets over an appropriate amplitude response threshold established by yield curve or data frequency distribution analysis. At Kaho'olawe, it was possible (and necessary) to discriminate basaltic decay response before target picking. Harvest selected decay curves for discrimination analysis.

e. Comparison of Decay Curves: Compute Chi-Squared measure of misfit from targets and bench calibration tests for expected ordnance items.

f. Prioritization of target list: Arrange in order of increasing chi-squared misfit.

Auto-leveling and target-picking are usually the most important and difficult data processing steps. At Kaho'olawe, the combination of excessive instrument drift and variable basalt background response made auto-leveling impossible. Details of the alternative EM-63 data processing and analysis steps as practiced at Kaho'olawe are as follows:

To remove the effects of the instrumental drift from the data and allow for variable basalt background decay response, a multistep procedure was used. First, the average decay curve of all points in a window (within $30 \mathrm{sec}$ and $20 \mathrm{~m}$ ) was subtracted from each individual decay curve in that window. These adjusted decay curves were then fit with the baseline basalt decay, producing a $\chi^{2}$ value, measuring the degree of misfit. The measurements were sorted by $\chi^{2}$, and the 
lowest 75 percent were reaveraged, producing a nominal drift baseline without the influence of measured anomalies. This baseline was then subtracted from the data points in the center $10 \mathrm{~m}$ of the window to produce the dedrifted data set, the windows were advanced by $10 \mathrm{~m}$, and the procedure was repeated.

This dedrifting produced a data set with both positive and negative basalt response, the former where the basalt level was higher than the local average, the latter where it was lower. Thus, the subsequent fits, which include a basalt component, allow that component to be positive or negative.

The dedrifted data were fit with a single basalt component first, producing a $\chi^{2}$ map with peaks where the instrument response was nonbasalt-like. These peaks were picked with standard GeoSoft software and were then turned into target lists. The discrimination of the targets was performed by simultaneously fitting both a basalt component and components from the ordnance library decay curves to the decay curve of the anomaly. This produced a $\chi^{2}$ for each type of ordnance in the library. These $\chi^{2}$ values were subtracted from the basalt-only $\chi^{2}$, and these $\Delta \chi^{2}$ values (corresponding to log-likelihood ratios in a model comparison test) were used to rank each target.

\section{Target Lists Provided to Environmental Security Technology Certification Program (ESTCP)}

Prioritized target lists were provided to ESTCP before leaving Maui, as required. It should be recognized, however, that a relatively larger coil geometry such as the 1-×1-m EM-63 is better for larger, deeper items, and not good for small, shallow items such as $20-\mathrm{mm}$ or thumb-sized fragmentation. This is a virtue, when small fragments are to be avoided. There is no single coil configuration that is optimum for all objects from $20-\mathrm{mm}$ to $155-\mathrm{mm}$ and large bombs.

\section{Acknowledgements}

NAEVA and GPA are very grateful for the opportunity to participate in the Kaho'olawe exercise, and for the excellent support provided by ERDC, NAVEODTECHDIV, ESTCP, Parsons, and the U.S. Navy. 


\section{E.2. Geophex Ltd.}

October 19, 2001

Naval EOD Tech Div

Indian Head, MD

Subject: Field Report on Kaho'olawe Demo

Attached disk contains the dig list in accordance with the format provided in the Demonstration Plan, dated August 31, 2001. Following brief descriptions apply:

- We employed 10 frequencies for this GEM-3 survey: 30; 150; 390; 750; 1,$470 ; 2,970 ; 5,910 ; 11,910 ; 23,850 ; 47,970 \mathrm{~Hz}$.

- The "signal strength" is derived by summing the differences in the quadrature responses among all frequencies. This was found to be most immune to the magnetic geology of the island.

- The signal strength was used for ranking the list.

- A threshold signal strength was used to derive the dig list without the 25-mm projectiles.

- The last column (added) denotes the western corner of the grid where each target belongs. For instance, $1 \mathrm{~A}$ denotes the grid bounded by Corner Post 1A, 1B, 2B, and 2A.

We attempted with difficulty to classify and identify each anomaly. In an attempt to minimize the library size (16 ESTCP Standard UXO and 13 Kaho'olawe UXO: a total of 29 UXO), we used the following eight groups. ES stands for the ESTCP Standard UXO and KH for the Kaho'olawe UXO provided at the QC Site.

$\begin{array}{llll}\text { Group 1 } & \text { Small } & \text { KH: } & \text { MT fuse } \\ \text { Group 2 } & \text { Small } & \text { ES: } & \text { 20-mm HE, 20-mm proj. M97, 40-mm MK2 } \\ \text { Group 3 } & \text { Small } & \text { KH: } & \text { BDU-3 submunition } \\ \text { Group 4 } & \text { Medium } & \text { ES: } & \text { 57-mm; 60-mm; 2.75-in. WHD w/o Fuse } \\ & & \text { KH: } & \begin{array}{l}\text { 60-mm canister, 81-mm ill w/fin, SMAW } \\ \text { rocket, 81-mm canister }\end{array} \\ & & & \text { 2.75-in. Warhead; 81-mm mortar } \\ \text { Group 5 } & \text { Medium } & \text { ES: } & \begin{array}{l}\text { 2.75-in. rocket, MK106 practice bomb, 2.25-in. } \\ \end{array} \\ & & \text { KH: } & \text { rocket, BDU33, 2.75-in. rocket } \\ \text { Group 6 } & \text { Medium } & \text { KH: } & \text { MK3 Practice bomb, LAW rocket motor } \\ \text { Group 7 } & \text { Medium } & \text { ES: } & \text { 60-mm; 76-mm; 81-mm mortar } \\ \text { Group 8 } & \text { Large } & \text { ES: } & \begin{array}{l}\text { 105-mm Apers; 4.-in. HE; 105-mm Heat, 5-in. } \\ \text { proj; 152-mm proj; 155-mm proj }\end{array}\end{array}$


Thank you for giving us this opportunity to participate in this challenging and interesting technology demonstration project.

Sincerely,

I.J. Won

Geophex, Ltd. 


\section{E.3. Naval Research Laboratory}

\section{MTADS Data Acquisition and Analysis Operations at Kaho'olawe}

\section{Data Acquisition}

The Calibration Site and the 30-m grids and 1-hectare grid on the QA Site were surveyed with the MTADS man-portable electromagnetic (EM) system; the Calibration site was also surveyed with the MTADS man-portable magnetometer system. The man-portable magnetometer array supports 2 Geometrics Model 822 sensors with a horizontal separation of $0.25 \mathrm{~m}$ and a height above ground of $0.20 \mathrm{~m}$. The EM platform used our current high-power single-gate version of the EM 61 with $0.5-\times 1.0-\mathrm{m}$ transmit and receive coils. The EM sensor was deployed with the long dimension cross-track at a transmitter coil height of $0.20 \mathrm{~m}$.

The Calibration site was marked in a North/South direction using twine on $1.5-\mathrm{m}$ spacing to define the survey lanes. Survey tracks were $0.5 \mathrm{~m}$ apart. The site was surveyed first with the EM system and then with the magnetometer platform.

The 30-m grids and the 1-hectare sites were marked as a single site in a Northeast/Southwest direction using twine on 1.5-m spacing. Survey tracks were $0.5 \mathrm{~m}$ apart. The $30-\mathrm{m}$ grids were completed in a single EM survey using survey tracks stretching the entire length of the site. The 1-hectare site was completed as a single EM survey, beginning at the northwest boundary.

The EM anomaly map for the Calibration Site is shown in Figure E3.1. It is presented as an interpolated image on a $175-\mathrm{mV}$ scale. The bright features stretching along the west and east boundaries are returns from $80 \mathrm{~d}$ steel nails that were used to stake the twine. These nails also served as timing fiduciary markers to calibrate the timing offsets for the EM system.

Figure E3.2 shows the EM anomaly Sitemap for the QA Site, including both the $30-\mathrm{m}$ grids and the 1-hectare area. This interpolated image is presented on a $1,000 \mathrm{mV}$ scale. About 50 of the brightest targets are readily apparent on this scale, as are the $80 \mathrm{~d}$ steel nails at the ends of the survey lines.

In all the EM presentations in this document, and for the purposes of our analysis, the EM data were smoothed with a 20-point "down-the-track" demedian filter. The GPS navigation data were smoothed with a 25-point filter, and the EM data contain a timing correction of $3 \mathrm{~ms}$. The demedian filter suppressed the larger-scale geological features without affecting the presentation or fitting for most UXO targets. During the EM analysis process, a data set was also displayed that was smoothed with a 1,000-point down-the-track filter. This data set was used when fitting larger UXO targets whose signatures were distorted by the much shorter scale 20-point filter. 


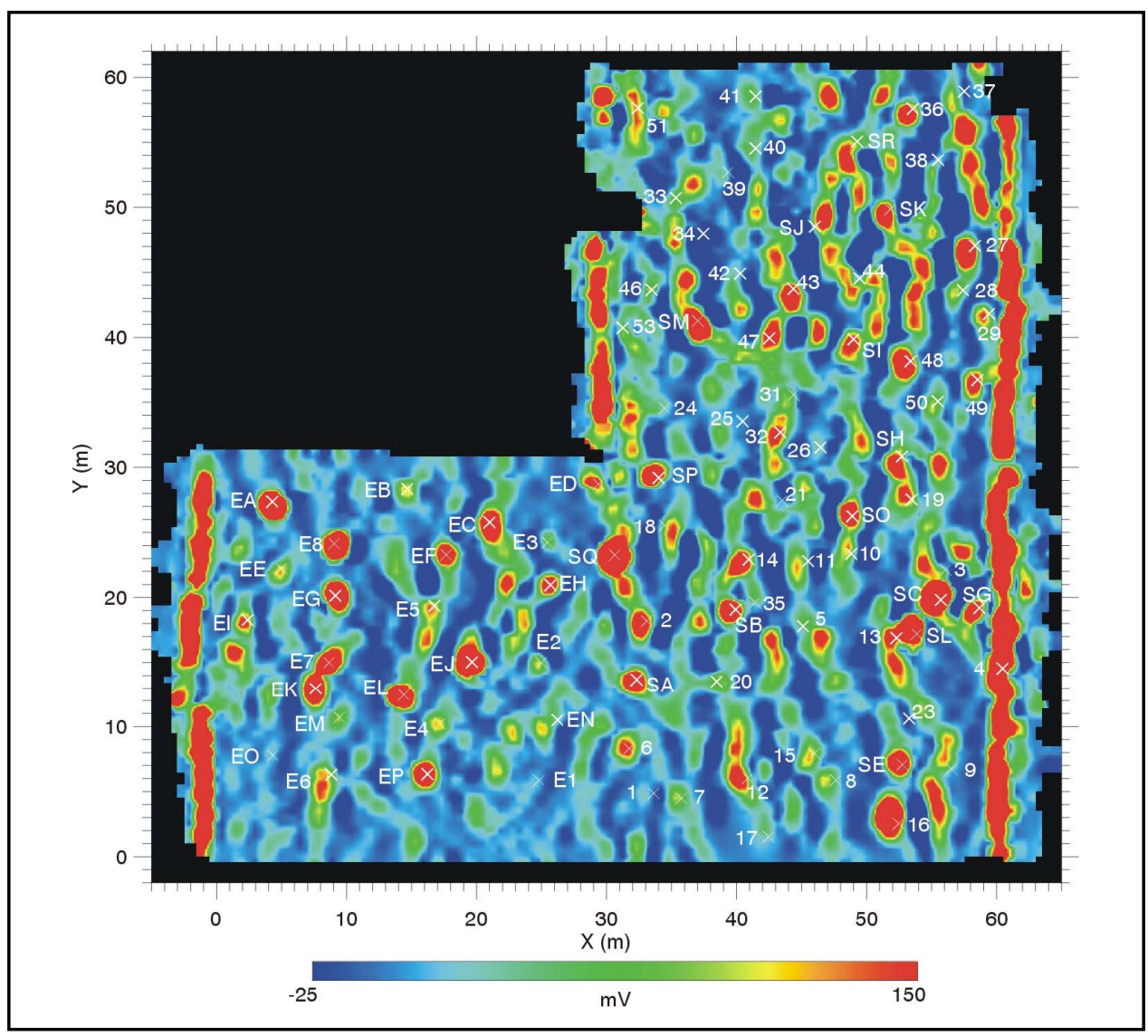

Figure E3.1. EM survey of the Kaho'olawe Calibration Grid. The presentation uses a 20-point demedian filter in an interpolated image

\section{Data Analysis}

\section{The Calibration Site}

Figure E3.3 shows the EM survey of the southwest third of the Calibration Site both as an interpolated image and as a pixel plot. Individual data points are sometimes apparent in the pixel plot. The overlay of the target locations and identities shows that most, but not all, targets are apparent in either presentation. In the Calibration Site the 20-point demedian filter effectively removed much of the interfering geological return. Table E3.1 shows a summary of our analysis of the Calibration Site, along with the groundtruth. The targets in the ESTCP Grid were much more detectible than the targets in the remainder of the site. Overall, in the Calibration Site we concluded that 43 of the 87 targets would likely not have been detected without knowledge of the groundtruth. The targets that would likely be missed in a blind EM survey are shaded in the right column in Table E3.1. Most of the large deep targets (projectiles or bombs) are buried below the detection limit of the instrument. The smaller targets, up to and including many of the 60- and 81-mm mortars would not be detected in a blind survey. The smaller targets were lost primarily because of the geological interference structure. The deepest targets would probably be beyond our detection limit, even without the geological. 


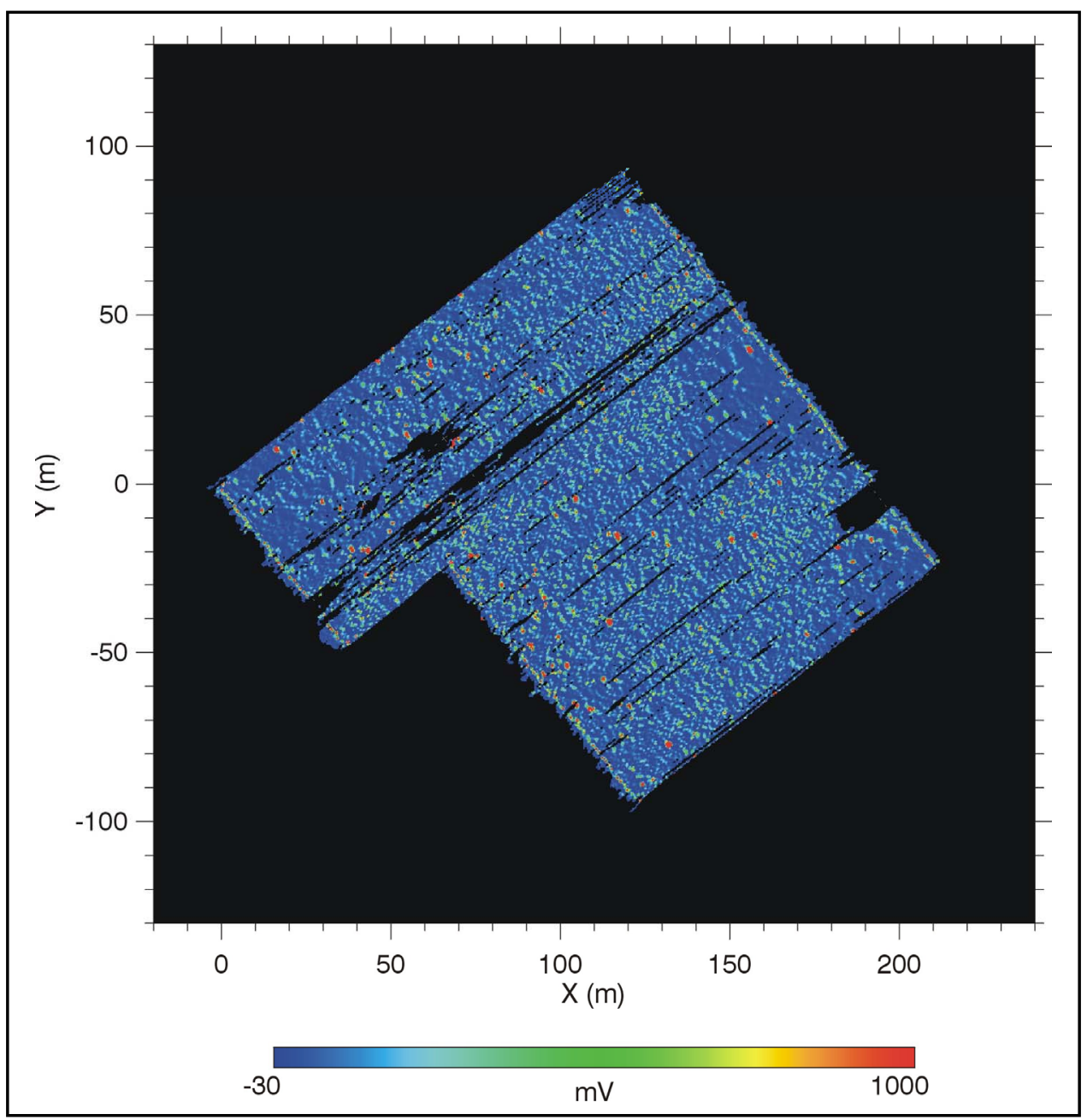

Figure E3.2. EM survey of the QA Site at Kaho'olawe. The presentation is an interpolated image, using a 20-point demedian filter. Note the different sensitivity scale from Figure E3.1

Figure E3.4 shows a presentation, on a similar area scale, of an anomaly image from the magnetometer survey. The geological interference is, of course, much worse in the magnetometry data than in the EM data. The data shown in Figure E3.4 are highly filtered (15 point, down-the-track demedian filter). Unfortunately, we did not have access to more sophisticated data processing filters (or the time to use them) to improve the magnetometer analysis. Many of the targets are apparent in the magnetometer data, however, only the deepest large targets were detectible in the magnetometer data but not in the EM data. In Figure E3.4 we have circled dipole signatures that are probably associated with the identified target assignments. The circled targets were declared as not detectible in the EM data. Because of time constraints, and because we did not have access or time to develop new data processing tools, we chose not to conduct magnetometry surveys of the QA Site. 


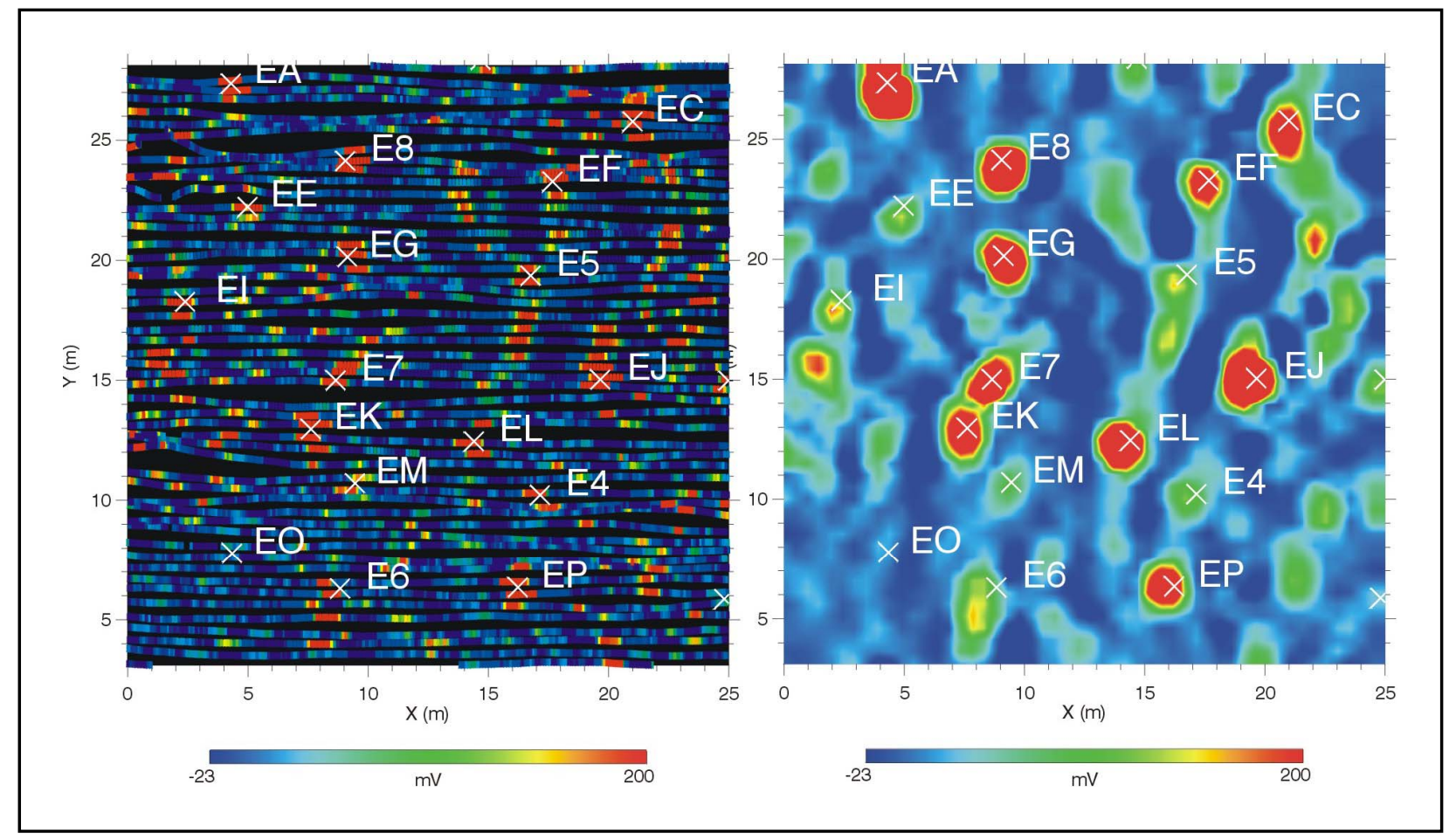

Figure E3.3. Southwest Quadrant of the Calibration Site. A comparison of pixel and interpolated image presentations of the EM survey are shown

\section{The QA Site}

The QA Site, shown in Figure E3.2, is much more highly disturbed than the Calibration Site. Figure E3.5 shows a pixel presentation of part of the 1E 30-m grid on about the same scale as the pixel plot in Figure E3.3. The entire QA Site looks similar when viewed on this presentation scale. There are three factors affecting the QA site that make analysis more difficult for us than on the Calibration Site. The density of small and intermediate sized shrapnel chunks is much higher on this site. In most cases, these present 0.5 - to 1-m-long singletrack signals, such as dominate the image in Figure E3.5. The surface of the QA site is also much rougher than the Calibration Site. Our EM sensors, unfortunately, generate sensor spikes when the system bumps hard over a surface feature. These signals, which one might expect to be sensor data spikes, unfortunately damp out over a period of a few tenths of a second, making them almost undistinguishable from the shrapnel clutter signal returns. Together, these two effects are responsible for most of the red signal return shown in Figure E3.5. This noise-dominated data set required that we had to carryout analysis at less sensitive scales, and give up even more of the small targets than in the Calibration Plot. The third effect, referred to above, is the more intense geological interference on this site. The geological returns effectively mitigated that we analyze data, with the visual guidance of the pixel presentations, rather than the combination of pixel and interpolated image presentations used at the Calibration Site. 


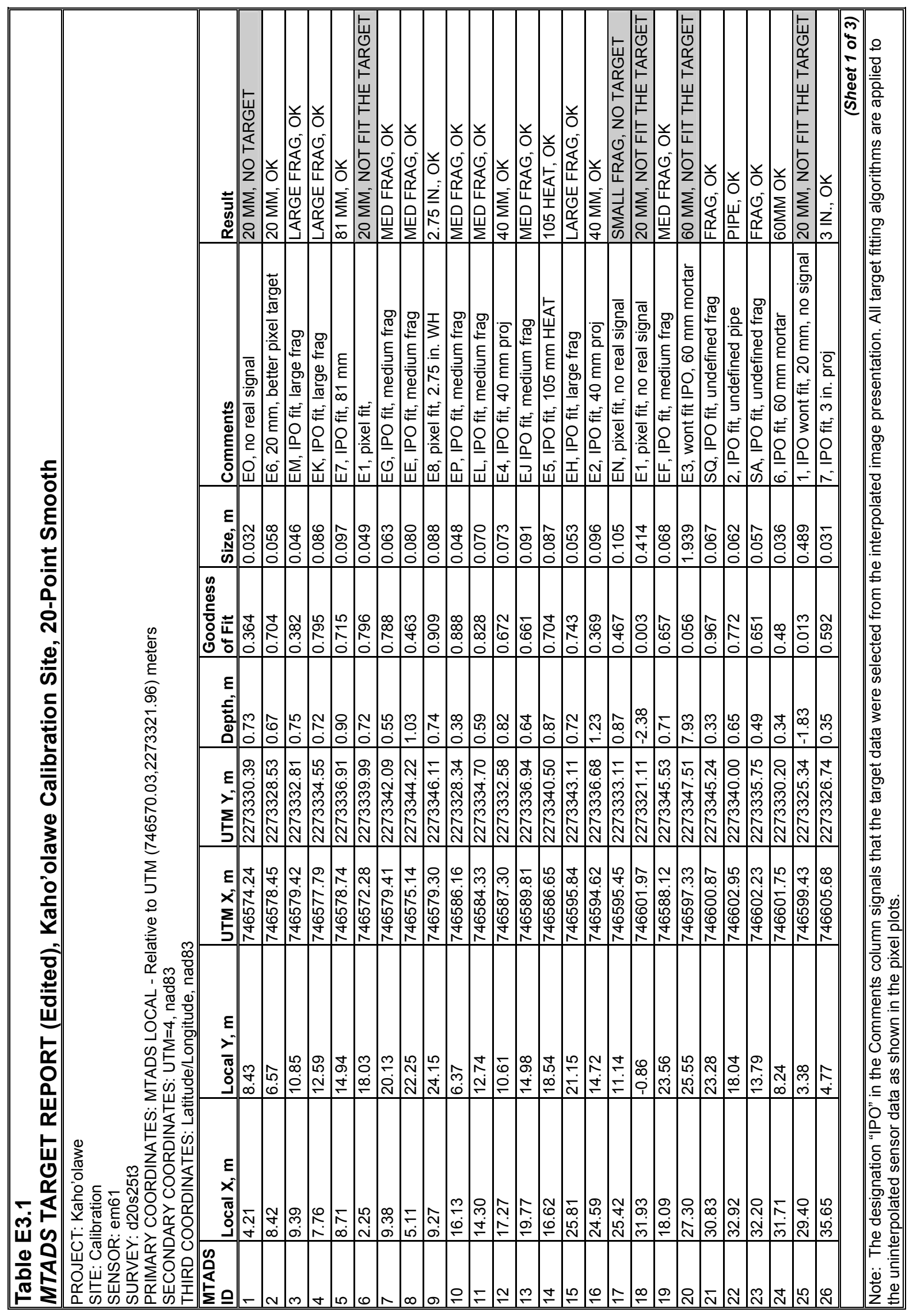




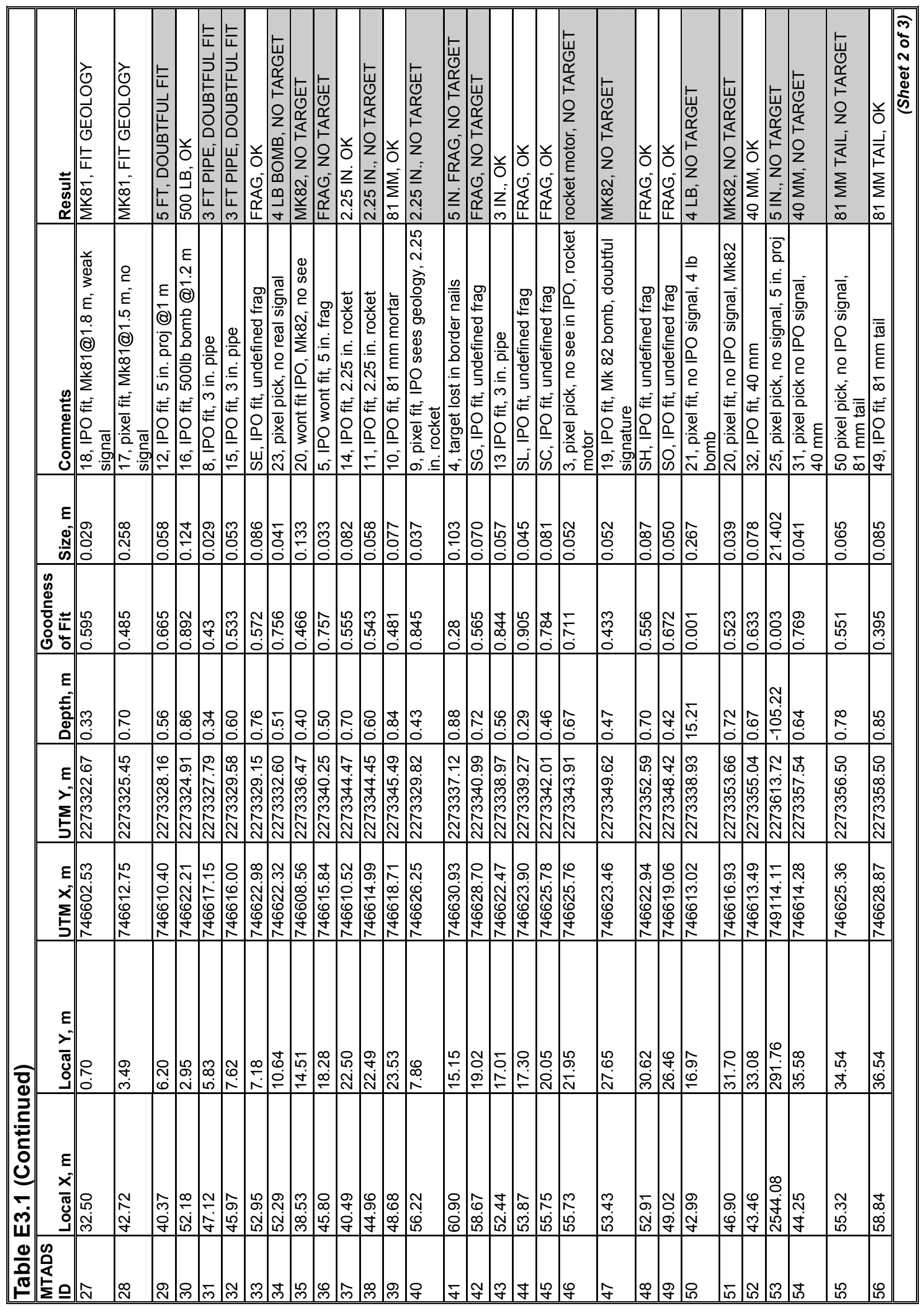




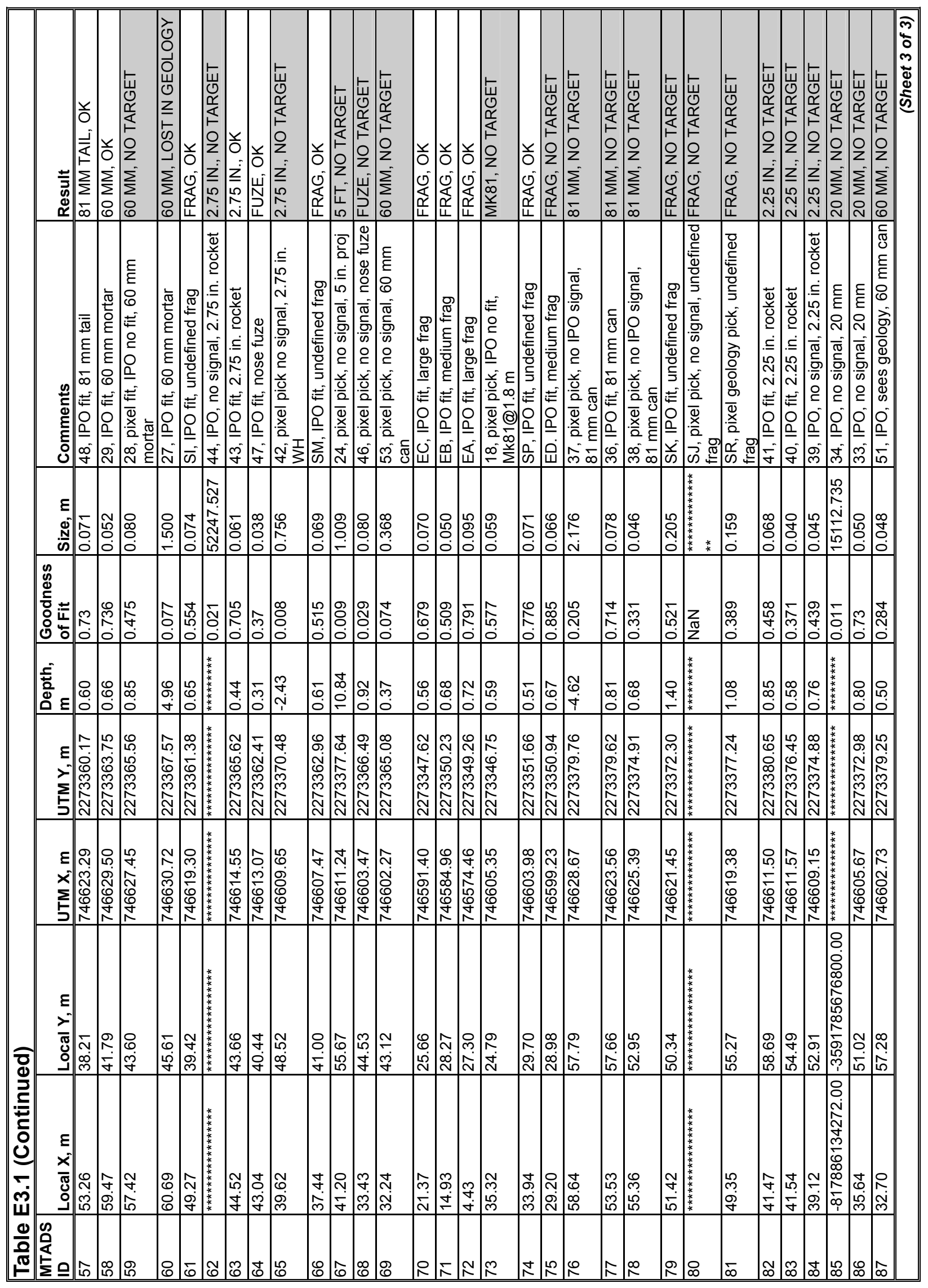




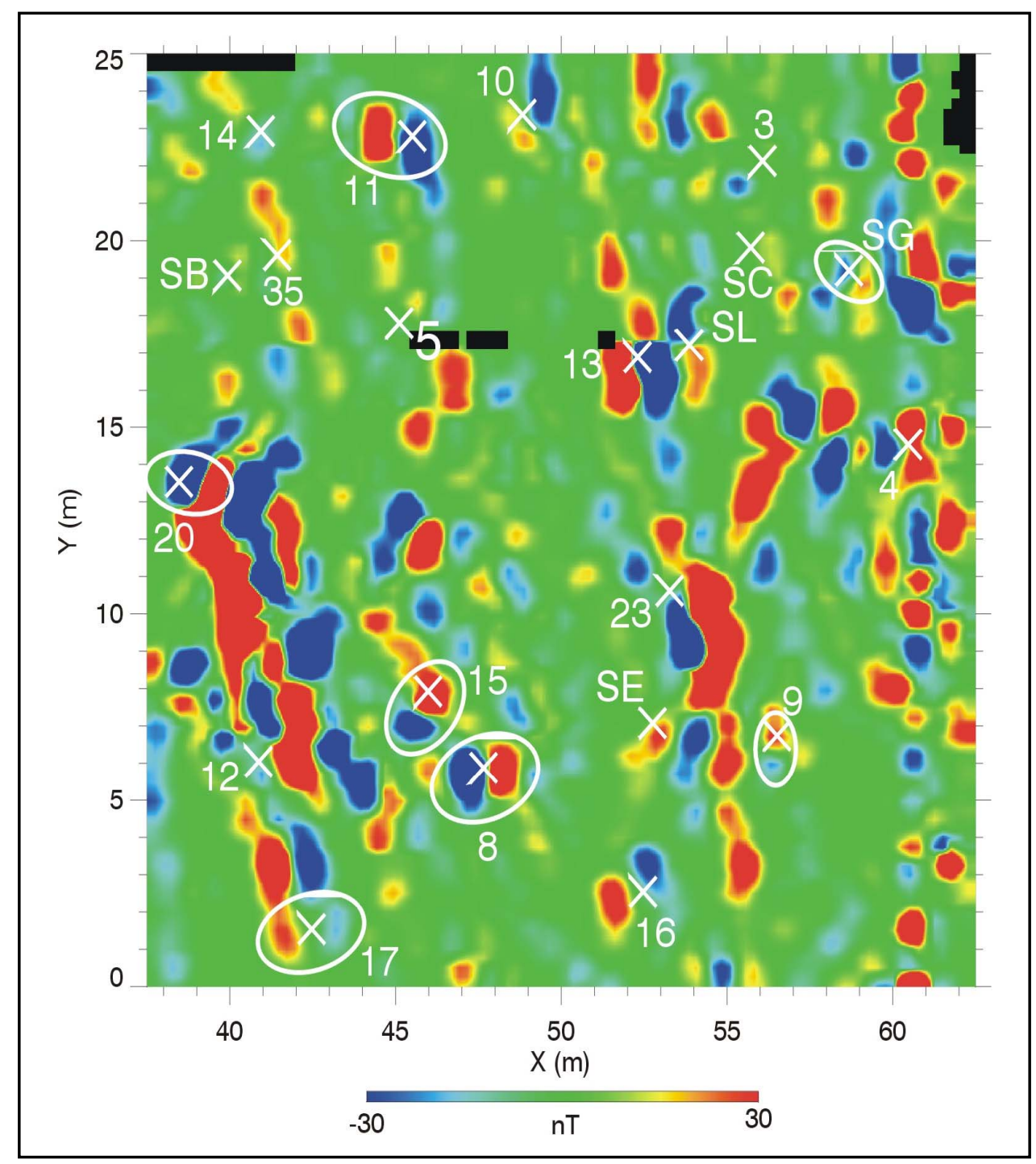

Figure E3.4. Calibration Site. Interpolated image from the magnetometry survey using a 15-point demedian filter

Our current EM data analysis uses what we call the three-beta analysis algorithm. This provides an analysis that generates three parameters that roughly correlate with size of the primary orthogonal dimensional axes of the target. We use these beta parameters as clues to target shape to provide information to use in classification decisions. The geological interferences, and the noise signals from the shrapnel clutter and sensor bouncing, rendered the three-beta parameters effectively useless as analysis tools. Therefore, the primary analysis took place using the previously developed baseline EM analysis approach. In this approach, important analysis parameters are location, depth, and size. Shape information, to the extent that it is available, is gleaned from the detrend presentation in the model fit analysis window. The "goodness of fit" parameter is used primarily as an evaluation tool to guide boxing the data chip for analysis and as a guide in editing the data selected for target fitting. 


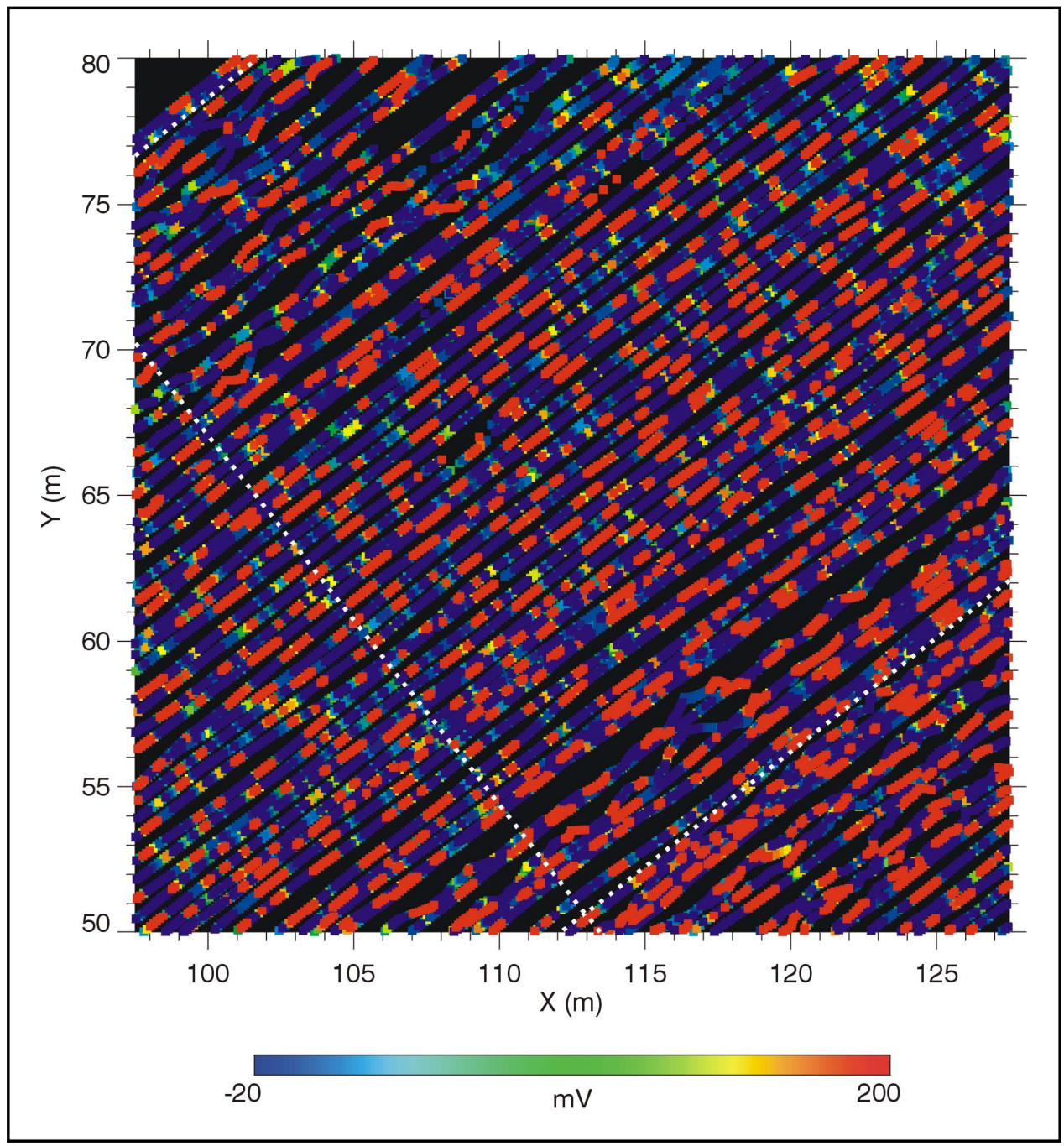

Figure E3.5. Pixel presentation of part of Grid 1E in the QA Site from the EM survey. The pixel presentation uses the same settings as the Calibration Site presentation shown in Figure E3.3

Presentations, such as shown in Figure E3.6 were used to guide the analysis. This presentation shows the targets chosen in Grid 1C. Target numbers are deleted so that the sensor data can be seen. To reduce the importance of shrapnel returns, we were guided by the upper coil data. We particularly sought out targets with returns in adjacent tracks on the assumption that larger targets would appear on multiple tracks. Some single-track targets were still reported. We typically rescaled the presentation many times to seek out smaller targets and to try to evaluate geological returns. However, because of the noise returns (such as shown in Figure E3.5) we were limited to working with fairly high intensity signals. We were aware that this mitigated against our detection of both small targets and the deep targets that had smaller intensity signal returns. 


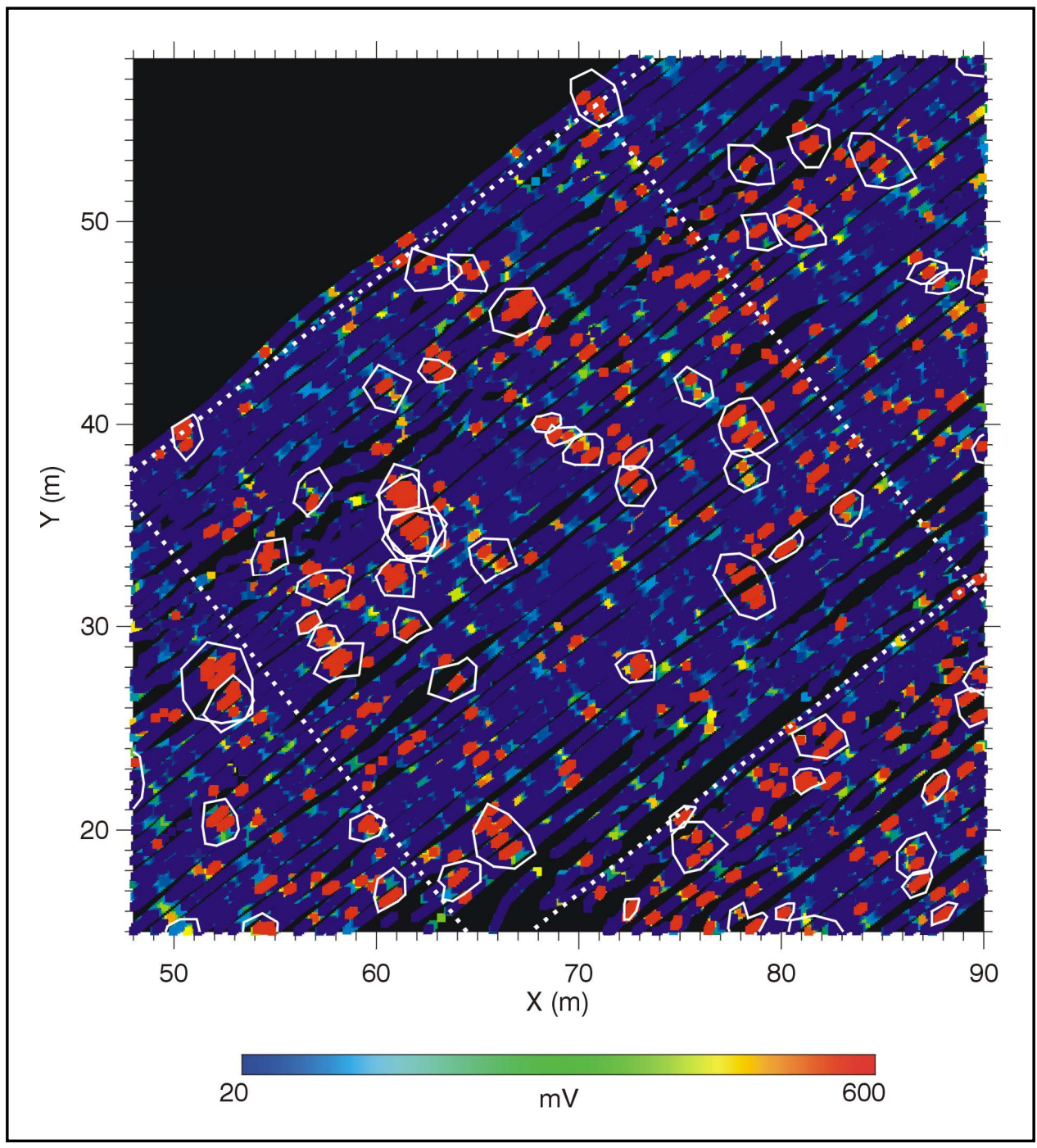

Figure E3.6. QA Site, Grid 1C. Pixel presentation of the EM survey showing targets selected for analysis 
E.4. Parsons Technology Inc.

SURVEY AND QUALITY ASSURANCE PLAN

\author{
PARSONS INFRASTRUCTURE \& \\ TECHNOLOGY
}

Advanced UXO Technology Discrimination/Discrimination
Technology Demonstration
Kaho'olawe Island, Hawaii

Naval Explosive Ordnance Disposal Technology Division

Research and Development Department

15 October, 2001 


\section{TABLE OF CONTENTS}

\subsection{DATA COLLECTION METHODS}

1.1 Geonics EM-61 Field Discrimination

1.2 Geophysical Technology Limited TM-5EMU Field Discrimination

1.3 Geonics EM-61 Postprocessed GPS-Integrated Digital Data

\subsection{SURVEY PROCEDURES}

2.1 Lane Spacing

2.2 Sampling Rates

2.3 Accuracy of Navigation and Tracking System

2.4 Data Format

\subsection{DATA COLLECTION PLAN}

\subsection{ANALYSIS AND DECISION PROCESS}

4.1 EM-61 Field Discrimination

4.2 EMU Field Discrimination

4.3 EM-61 Postprocessed GPS Integrated Digital Data

\subsection{PERSONNEL INFORMATION}




\subsection{Data Collection Methods}

\subsection{Geonics EM-61 Field Discrimination}

The first method employed by Parsons Infrastructure and Technology (Parsons) will be a field discrimination ("mag \& flag") survey utilizing a standard Geonics EM-61 (EM-61) full meter coil (1 m by $1 \mathrm{~m})$.

\subsection{Geophysical Technology Limited TM-5EMU Field Discrimination}

The second method employed by Parsons will be a field discrimination ("mag \& flag") survey utilizing a standard Geophysical Technology Limited (GTL) TM-5EMU (EMU).

\subsection{Geonics EM-61 Postprocessed GPS-Integrated Digital Data}

The third method employed by Parsons will be a survey utilizing a Geonics EM-61 postprocessed GPS-integrated digital data system. This method will be employed only if time constraints allow (i.e., the first two methods have been completed with enough time remaining to allow digital data collection).

Surveys to be independent of each other

All three surveys will be conducted independently of each other utilizing separate teams.

\subsection{Survey Procedures}

\subsection{Lane Spacing}

EM-61 and EM-61 Digital Data: For the EM-61 field discrimination and postprocessed GPS-integrated digital data surveys, a 1-m lane spacing will be utilized. The EM-61 coil is 1-m wide, which allows a 1.5- to 2-m-wide effective survey path (dependent on target depth, orientation, composition, and size). The overlap will ensure that sufficient coverage of the test grids is obtained.

EMU Lane Spacing: For the EMU field discrimination survey, a 1-m lane spacing will be utilized. The standard EMU has a 0.5 -m-diameter coil. The sweep range (lateral arc) of our operators ranges between 1.5 and $2 \mathrm{~m}$. The overlap created by the sweep range in conjunction with the $1-\mathrm{m}$ lane path will ensure that sufficient coverage of the test grid is obtained. 


\subsection{Sampling Rates}

EM-61 and EM-61 Digital Data: For the EM-61 field discrimination and postprocessed GPS-integrated digital data surveys, the EM-61 will be set in the "Auto Mode, Extra Fast Mode." Extra fast mode results in 7 readings per second. The Ashtech Reliance GPS system records GPS positional readings at a minimum rate of 30 readings per minute.

EMU: The EMU takes 58 samples per second.

\subsection{Accuracy of Navigation and Tracking System}

The navigation system used during collection for all three survey methods will be a visual system utilizing cones and tapes. Tapes will be placed approximately every $30 \mathrm{~m}$ perpendicular to the travel direction. Cones will be placed on each tape at the current lane. Given the relative flatness of the terrain comprising the test grids, this navigational system will provide less than $0.5 \mathrm{~m}$ of lateral offset from the intended line path. During field discrimination, each anomaly will be investigated in a perpendicular direction to the travel lane, which will result in a radial error of less than $0.5 \mathrm{~m}$.

An Ashtech Z-Surveyor GPS system will be utilized to record the position of each anomaly detected in both the EM-61 field discrimination survey and the TM-5 EMU field discrimination survey. The real-time accuracy of this system is $\pm 0.01 \mathrm{~m}$.

An Ashtech Reliance GPS system will be utilized for the EM-61 digital data survey. The postprocessed accuracy of this system is $\pm 0.1 \mathrm{~m}$.

\subsection{Data Format}

Data will be submitted in an ASCII format that includes sensor location data in UTM coordinates, followed by sensor readings. Header information required under Section 6.1 of the Technology Demonstration Plan will also be included in the data files.

\subsection{Data Collection Plan}

Parsons plans to collect the EM-61 field discrimination and the EMU field discrimination simultaneously. One team will begin collecting data in the $30-\mathrm{m}$ by $30-\mathrm{m}$ grids and a second team will concurrently begin collecting data in the one-hectare grid. Real-time surveyor(s) will simultaneously survey anomaly locations. Parsons will not be seeking groundtruth for the first four $30-\mathrm{m}$ by $30-\mathrm{m}$ grids prior to completing the surveys.

Following completion of the field discrimination surveys, the EM-61 digital data collection will commence. 


\subsection{Analysis and Decision Process}

\subsection{EM-61 Field Discrimination}

Trained and certified EM-61 operators will analyze the tonal and numeric response of the EM-61 in real-time along 1-m-wide lanes. Each 5-mV rise above the local background reading will be investigated as a possible anomaly. The operator will investigate each potential anomaly perpendicular to the lane path as well as along the path. Anomalies will be identified based on the amplitude, slope, wavelength, and shape of the EM-61 response in perpendicular directions.

\subsection{EMU Field Discrimination}

Trained and certified EMU operators will analyze the tonal characteristics, graphical characteristics, and numeric response of the instrument in real time along 1-m-wide lanes. Each EMU response above the local background reading will be investigated as a possible anomaly. Anomalies will be identified based on the amplitude, slope, wavelength, shape, and size of the EMU response.

\subsection{Geonics EM-61 Postprocessed GPS Integrated Digital Data}

Trained and certified EM-61 operators will utilize an EM-61 and an Ashtech Reliance GPS receiver to simultaneously record digital EM61 response and GPS positional data along 1-m-wide lanes. The EM-61 data and postprocessed GPS positional data will be synchronized through their recorded time stamps. These data will be downloaded and analyzed offsite by a trained and certified data analyst. The GPS data will be postprocessed using GPS data from an on-site base station and merged with the EM-61 data. The resulting files (containing easting, northing, and electromagnetic response) will then be analyzed using Geosoft's Oasis Montaj software. The data analyst will apply a lag correction to the data based on the analysis of a field-conducted daily instrument test. The analyst will create profiles of the EM-61 response along each lane for both the top coil and the bottom coil. Grid maps will be produced using the raw EM-61 data as well as data run through high pass, vertical derivative, and analytic signal filters. The data analyst will investigate lane profiles, 2-dimensional maps and 3-dimensional maps to identify anomalies based on a minimum 5-mV EM-61 response above the background, in conjunction with the slope, wavelength, and shape of the response from each coil. 


\subsection{Personnel Information}

\section{EM-61 Field Discrimination}

\begin{tabular}{|l|l|}
\hline Type of Work & Personnel \\
\hline Field Discrimination & Richard Arts \\
\hline Field Discrimination & Addie Johansen \\
\hline GPS Surveying & Tom Dickson \\
\hline
\end{tabular}

EMU Field Discrimination

\begin{tabular}{|l|l|}
\hline Type of Work & Personnel \\
\hline Field Discrimination & Jon Lincoln \\
\hline Field Discrimination & Michael Ault \\
\hline GPS Surveying & Kamalei Hill \\
\hline
\end{tabular}

EM-61 Digital Data Collection and Processing

\begin{tabular}{|l|l|}
\hline Type of Work & Personnel \\
\hline Data Collection & Dean Tokishi \\
\hline Data Collection & Steve Vuich \\
\hline Processing Geophysicist & Andy Gascho \\
\hline
\end{tabular}




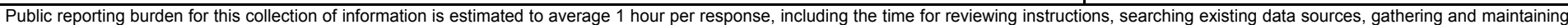

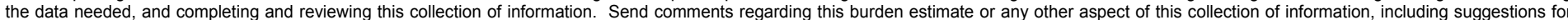

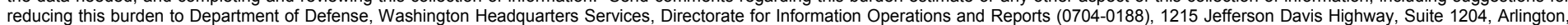

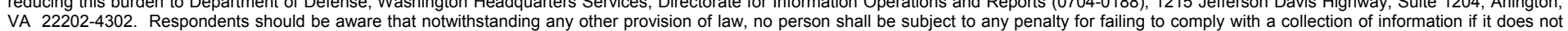
VA 22202-4302. Respondents should be aware that notwithstanding any other provision of law, no person shall be sube
display a currently valid OMB control number. PLEASE DO NOT RETURN YOUR FORM TO THE ABOVE ADDRESS.

\begin{tabular}{l|c}
$\begin{array}{l}\text { 1. REPORT DATE (DD-MM-YYYY) } \\
\text { March } 2004\end{array}$ & $\begin{array}{c}\text { 2. REPORT TYPE } \\
\text { Final report }\end{array}$ \\
\hline
\end{tabular}

\section{TITLE AND SUBTITLE}

Advanced UXO Detection/Discrimination Technology Demonstration,

Kaho'olawe, Hawaii

3. DATES COVERED (From - To)

5a. CONTRACT NUMBER

5b. GRANT NUMBER

5c. PROGRAM ELEMENT NUMBER

\section{AUTHOR(S)}

Diane M. Cargile, Hollis H. Bennett, Ricky A. Goodson, Tere’ A. DeMoss, Ernesto R. Cespedes

\section{5d. PROJECT NUMBER}

200034 \& AF-25

5e. TASK NUMBER

5f. WORK UNIT NUMBER

008WGQ

\section{PERFORMING ORGANIZATION NAME(S) AND ADDRESS(ES)}

8. PERFORMING ORGANIZATION REPORT NUMBER

U.S. Army Engineer Research and Development Center

Environmental Laboratory

ERDC/EL TR-04-1

3909 Halls Ferry Road

Vicksburg, MS 39180-6199

\section{SPONSORING / MONITORING AGENCY NAME(S) AND ADDRESS(ES)}

10. SPONSOR/MONITOR'S ACRONYM(S)

Environmental Security Technology Certification Program Office

Arlington, VA 22203;

Headquarters, U.S. Army Corps of Engineers

Washington, DC 20314-1000

11. SPONSOR/MONITOR'S REPORT NUMBER(S)

\section{DISTRIBUTION / AVAILABILITY STATEMENT}

Approved for public release; distribution is unlimited.

\section{SUPPLEMENTARY NOTES}

\section{ABSTRACT}

The primary technical objective of this demonstration project was to evaluate the detection and discrimination capabilities (including production rates and costs) of advanced UXO systems in difficult magnetic clutter environments such as those encountered at Kaho'olawe, Hawaii. One 90-m by 111.1-m (1-hectare) area and 10 (not necessarily contiguous) 30-m by 30-m test grids within the Kaho'olawe Quality Assurance (QA) Range were prepared to present a limited range of target/clutter/topography/vegetation/magnetic background conditions to the various demonstrators' systems: Geonics EM-63, GTL TM-5 EMU, Geophex GEM-3, NRL EMMS, and Geonics EM-61. Anomaly maps, survey maps, and demonstrators target discrimination charts are compared to actual groundtruth to determine performance assessment of detection, discrimination, and false alarm rate. At Kaho'olawe, the advanced EMI systems did not demonstrate significant performance and/or cost improvements over the baseline technology consisting of a standard EM-61 system operated in an "EM and Flag" mode. This was not true at Jefferson Proving Ground, Indiana (July 2000). Finally, the safety and logistics problems associated with conducting technology demonstrations concurrent with actual UXO cleanup operations proved to be a very inefficient, costly, and time-consuming process.

\section{SUBJECT TERMS}

Electromagnetic

Frequency-domain system

16. SECURITY CLASSIFICATION OF:

\begin{tabular}{|l|l|}
\hline a. REPORT & b. ABSTRACT \\
UNCLASSIFIED & UNCLASSIFIED \\
\hline
\end{tabular}

\section{Magnetic clutter}

Magnetometry

Multi-channel time domain system

c. THIS PAGE
UNCLASSIFIED

17. LIMITATION OF ABSTRACT

\section{Target detection}

Target discrimination

UXO
18. NUMBER $19 a$. NAME OF RESPONSIBLE OF PAGES

187

\section{PERSON}

19b. TELEPHONE NUMBER (include area code) 